\title{
MAXIMAL FUNCTIONS ASSOCIATED WITH NONISOTROPIC DILATIONS OF HYPERSURFACES IN $\mathbb{R}^{3}$
}

\author{
Dissertation \\ zur Erlangung des Doktorgrades \\ der Mathematisch-Naturwissenschaftlichen Fakultät \\ der Christian-Albrechts-Universität zu Kiel
}

vorgelegt von

Wenjuan Li

Kiel, 2015 

Erster Gutacher: Prof. Dr. Detlef Müller

Zweiter Gutacher: Priv.-Doz. Dr. Michael Gnewuch

Tag der mündlichen Prüfung: 22.06.2015

Zum Druck genehmigt: 22.06.2015

gez. Prof. Dr. Wolfgang J. Duschl, Dekan 



\section{Abstract}

The goal of this thesis is to establish $L^{p}$-estimates for maximal functions associated with nonisotropic dilations of hypersurfaces in $\mathbb{R}^{3}$. More precisely, let $S$ be a smooth hypersurface in $\mathbb{R}^{3}$ with a surface measure $d \mu$ and $\eta \in C_{0}^{\infty}\left(\mathbb{R}^{3}\right)$ be a smooth nonnegative function with compact support. Suppose that $\delta_{t}(x)=\left(t^{a_{1}} x_{1}, t^{a_{2}} x_{2}, t^{a_{3}} x_{3}\right)$ is a family of dilations with $a_{j}>0$. Then the associated maximal operator is given by

$$
\mathcal{M} f(x):=\sup _{t>0}\left|\int_{S} f\left(x-\delta_{t}(y)\right) \eta(y) d \mu(y)\right|, \quad f \in \mathcal{S}\left(\mathbb{R}^{3}\right) .
$$

The problem of characterizing the range of all $p$ for which $\mathcal{M}$ is bounded on $L^{p}\left(\mathbb{R}^{3}\right)$ has been extensively considered. The best understood class are maximal functions associated with isotropic standard dilations $\delta_{t}(x)=t x$, see the articles by Greenleaf, Iosevich-Sawyer, Ikromov-Kempe-Müller and Zimmermann. Some results for maximal functions associated with nonisotropic dilations have already been obtained by Greenleaf, Iosevich-Sawyer and Zimmermann, but for some situations such as the hypersurface parameterized as the graph of a smooth function $\Phi\left(x_{1}, x_{2}\right)=x_{2}^{d}\left(1+\mathcal{O}\left(x_{2}^{m}\right)\right)$ near the origin, where $d \geq 2, m \geq 1$, and associated dilations $\delta_{t}(x)=\left(t^{a} x_{1}, t x_{2}, t^{d} x_{3}\right)$ for an arbitrary real number $a>0$, the question was open until recently. In fact, such problems do arise already in lower dimensions. For instance, we consider the curve $\gamma(x)=\left(x, x^{2}(1+\phi(x))\right)$, where $\phi(x)=\mathcal{O}\left(x^{m}\right)$ and $m \geq 1$, and associated dilations $\left(x_{1}, x_{2}\right) \rightarrow\left(t x_{1}, t^{2} x_{2}\right)$. If $\phi \equiv 0$, then the corresponding maximal function is the maximal function along parabolas in the plane, which plays an important role in the study of singular Randon transforms, and which is very well understood due to the work by Nagel-Riviere-Wainger and others. If $\phi \neq 0$, the problem was open until recently, however, the corresponding maximal function shows features related to the Bourgain circular maximal function, which required deep ideas and $L^{4}$-techniques. An alternative approach was later given by Mockenhaupt, Seeger and Sogge, who established local smoothing estimates for Fourier integral operators satisfying the so-called "cinematic curvature" condition. However, we observe that in the study of $\mathcal{M}$ related 
to the mentioned curve $\gamma(x)$ and associated dilations, in our situation, we will consider a family of corresponding Fourier integral operators which fail to satisfy the "cinematic curvature condition" uniformly, which means that classical local smoothing estimates could not be directly applied to our problem.

In this thesis, we develop new ideas in order to overcome the above difficulty and finally establish sharp $L^{p}$-estimates for the maximal function related to the curve $\gamma(x)$ with associated dilations in the plane. Later, we generalize the result to curves of finite type $d(d \geq 2)$ and associated dilations $\left(x_{1}, x_{2}\right) \rightarrow\left(t x_{1}, t^{d} x_{2}\right)$. Furthermore, we also obtain $L^{p}$-estimates for the maximal function related to the mentioned hypersurface $\Phi\left(x_{1}, x_{2}\right)$ in $\mathbb{R}^{3}$ with associated dilations. Moreover, by an alternative approach, we also get $L^{p}$-estimates for some classes of maximal functions in $\mathbb{R}^{3}$ established earlier by Greenleaf, Iosevich-Sawyer, Ikromov-Kempe-Müller and Zimmermann. 


\section{Zusammenfassung}

Das Ziel dieser Arbeit ist es, $L^{p}$-Abschätzungen für Maximalfunktionen zu nichtisotropen Dilatation von Hyperflächen im $\mathbb{R}^{3}$ zu untersuchen. Genauer gesagt, sei $S$ eine glatte Hyperfläche im $\mathbb{R}^{3}$ mit Oberflächenmaß $d \mu$ und sei $\eta \in C_{0}^{\infty}\left(\mathbb{R}^{3}\right)$ eine glatte nichtnegative Funktion mit kompaktem Träger. Angenommen, $\delta_{t}(x)=\left(t^{a_{1}} x_{1}, t^{a_{2}} x_{2}, t^{a_{3}} x_{3}\right)$ ist eine Familie von Dilatation. Dann ist der zugehörige Maximaloperator gegeben durch

$$
\mathcal{M} f(x):=\sup _{t>0}\left|\int_{S} f\left(x-\delta_{t}(y)\right) \eta(y) d \mu(y)\right|, \quad f \in \mathcal{S}\left(\mathbb{R}^{3}\right) .
$$

Die Frage nach dem Bereich aller $p$, für die $\mathcal{M}$ auf $L^{p}\left(\mathbb{R}^{3}\right)$ beschränkt ist, wurde intensiv studiert. Die am besten verstandene Klasse sind Maximalfunktionen zu den isotropen Standard-Dilatation $\delta_{t}(x)=t x$, siehe die Artikel von Greenleaf, Iosevich-Sawyer, Ikromov-Kempe-Müller und Zimmermann. Für Maximalfunktionen zu nichtisotropen Dilatation gibt es einige Resultate von Greenleaf, Iosevich, Sawyer und Zimmermann, aber für einige Situationen, wie zum Beispiel die Hyperfläche $\Phi\left(x_{1}, x_{2}\right)=x_{2}^{d}\left(1+\mathcal{O}\left(x_{2}^{m}\right)\right)$, wobei $d \geq 2, m \geq 1$, und zugehörigen Dilatation $\delta_{t}(x)=\left(t^{a} x_{1}, t x_{2}, t^{d} x_{3}\right)$ für eine beliebige reelle Zahl $a>0$, war die Frage bislang offen. In der Tat treten analoge Probleme schon in niedriger Dimension auf. Betrachten wir zum Beispiel die Kurve $\gamma(x)=\left(x, x^{2}(1+\phi(x))\right)$, wobei $\phi(x)=\mathcal{O}\left(x^{m}\right)$ und $m \geq 1$, und die damit verbundenen Dilatation $\left(x_{1}, x_{2}\right) \rightarrow\left(t x_{1}, t^{2} x_{2}\right)$. Für $\phi \equiv 0$ ist die entsprechende Maximalfunktion gerade die Maximalfunktion entlang Parabeln in der Ebene, die im Studium von sin-

gulären Randon-Transformationen eine wichtige Rolle spielt, und die aufgrund von Arbeiten von Stein, Nagel-Riviere-Wainger und anderen sehr gut verstanden sind. Für $\phi \neq 0$ zeigt die entsprechende Maximalfunktion Charakteristiken des Bourgainschen Maximaloperators für Kreise, diese Behandlung tiefliegende Ideen und $L^{4}$-Techniken erforderten. Ein alternativer Ansatz von Mockenhaupt, Seeger und Sogge beruht auf sogenannten "local smoothing"-Abschätzungen für Fourier-Integraloperatoren, die gewissen "kinematischen" Krümmungsbedingungen genügen. Wir werden sehen, dass beim Studium des Maximaloperators zu der erwähnten Kurve $\gamma(x)$ und zugehörige Dilata- 
tion die Familie der entsprechenden Fourier-Integraloperatoren nicht geleichmäßig der "kinematischen" Krümmungbedingung genügt, was bedeutet, dass die klassische sogenannte "local smoothing"-Abschätzung nicht direkt auf unser Problem angewendet werden kann.

In dieser Arbeit entwickeln wir neue Ideen, um die obigen Schwierigkeiten zu überwinden und schließlich die scharfe $L^{p}$-Abschätzung für die Maximalfunktion zur Kurve $\gamma(x)$ und die verbundenen Dilatationen in der Ebene zu beweisen. Später verallgemeinern wir das Resultat für Kurven vom endlichen Typ $d(d \geq 2)$ und zugehörige Dilatation $\left(x_{1}, x_{2}\right) \rightarrow\left(t x_{1}, t^{d} x_{2}\right)$. Wir erhalten die $L^{p}$-Abschätzung auch für die Maximalfunktion der genannten Hyperfläche $\Gamma\left(x_{1}, x_{2}\right)$ im $\mathbb{R}^{3}$ mit die verbundenen Dilatation. Darüber hinaus geben wir einen alternativen Beweis für $L^{p}$-Abschätzungen einiger Klassen von Maximalfunktionen im $\mathbb{R}^{3}$, die von Greenleaf, Iosevich-Sawyer, Ikromov-Kempe-Müller und Zimmermann bereits mit anderen Methoden gezeigt wurden. 


\section{Danksagung}

Ich möchte meine tiefste Dankbarkeit meinem Betreuer Prof. Dr. D. Müller für seine ständige Hilfe aussprechen. Ohne seine geduldigen Hinweise, aufschlussreiche Kritik und fachmännisch Unterstützung wäre die Fertigstellung dieser Arbeit nicht möglich gewesen.

Ich bin auch Prof. Dr. H. König und Dr. A. Martini zu Dank verpflichtet, von deren Lehre und aufschlussreichen Vorträgen ich viel profitiert habe. Ganz besonders danke ich Dr. A. Martini für ausführliche Diskussionen in der Studienzeit.

Vielen Dank an Dr. S. Buschenhenke, Dr. T. R. Johansen, Dr. M. Song und Dr. E. Zimmermann, die mir wertvolle Anregungen im Studium geboten haben und viel Zeit verbrachten, um diese Arbeit zu überprüfen.

感谢三年多来陪伴在我身边的同学和朋友们, 正是你们刻苦学习的精神以及快乐的生活态度 感染着我, 让我在基尔学习的日子特别充实。最后, 感谢我的父母以及家人, 特别是我的爱 人, 对我学术追求的理解和支持。 



\section{Contents}

$\begin{array}{ll}\text { Abstract } & \text { iii }\end{array}$

Zusammenfassung v

Danksagung _ vii

1 Introduction $\quad 1$

1.1 Background on maximal functions . . . . . . . . . . . . . . . 2

1.2 Local smoothing estimates . . . . . . . . . . . . . . 4

1.3 Organization of the thesis . . . . . . . . . . . . 7

2 Maximal functions associated with nonisotropic dilations

of curves in the plane $\quad 9$

2.1 Statement of the main results . . . . . . . . . . . . . . . 10

2.2 Background on Fourier integral operators and auxiliary results . . . . . 13

2.2.1 Local smoothing of Fourier integral operators . . . . . . . . . 13

2.2.2 Auxiliary results . . . . . . . . . . . . . . . 15

2.3 The proof for curves with non-vanishing Gaussian curvature . . . . . . 17

2.3.1 The case when $m=1 \ldots \ldots \ldots$. . . . . . . . . . 18

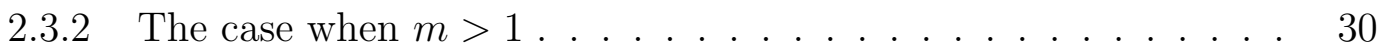

2.4 The proof for curves of finite type . . . . . . . . . . . . . 33

$\begin{array}{lll}3 & \text { A model operator } & 37\end{array}$

4 More general Fourier integral operators not satisfying

the cinematic curvature condition uniformly 
5 Maximal functions associated with nonisotropic dilations of $\begin{array}{ll}\text { some classes of hypersurfaces in } \mathbb{R}^{3} & 69\end{array}$

5.1 Statement of the main results . . . . . . . . . . . . . . 70

5.2 Proofs for surfaces with one non-vanishing principal curvature . . . . . 72

5.2 .1 Maximal theorem with $2 a_{2} \neq a_{3} \ldots \ldots . \ldots 72$

5.2.2 Maximal theorem with $2 a_{2}=a_{3} \ldots \ldots$. . . . . . . . 81

5.3 Proofs for surfaces of finite type . . . . . . . . . . . . 85

5.3.1 Maximal function theorem with $d a_{2} \neq a_{3} \ldots \ldots . . . . . .85$

5.3.2 Maximal theorem with $d a_{2}=a_{3} \ldots \ldots$. . . . . . . . 86

5.4 Proofs for surfaces not passing through the origin . . . . . . . . . . 86

$\begin{array}{ll}\text { Bibliography } & 91\end{array}$

$\begin{array}{ll}\text { Erklärung } & 95\end{array}$ 
Chapter 1

\section{Introduction}




\subsection{Background on maximal functions}

If $f: \mathbb{R}^{n} \rightarrow \mathbb{C}$ is a locally integrable function, the classical Hardy-Littlewood maximal function $M f: \mathbb{R}^{n} \rightarrow \mathbb{C}$ is defined by

$$
M f(x):=\sup _{r>0} \frac{1}{|B(x, r)|} \int_{B(x, r)}|f(y)| d y,
$$

where $B(x, r)$ is the ball of radius $r$ centered at $x$, and $|E|$ denotes the measure of a measurable set $E$. It arises very naturally in analysis, for proving theorems about the existence almost everywhere of limits, for controlling pointwise important objects such as the Poisson integrals or for controlling, not pointwise but at least in average, other basic operators such as singular integral operators. For example, by a standard density argument, the weak $(1,1)$ boundedness of $M$ implies in particular that Lebesgue differentiation theorem, according to which

$$
\lim _{r \rightarrow 0} \frac{1}{|B(x, r)|} \int_{B(x, r)} f(y) d y=f(x)
$$

for all $f \in L^{1}\left(\mathbb{R}^{n}\right)$ and almost every $x \in \mathbb{R}^{n}$.

In the past 40 years, the interest in taking averages over low dimensional sets has been increasing. E.g. in (1.1) one replaces the balls of radius $r$ by a suitable family of lowerdimensional set. The study of such averages is of interest not only for its own sake, but because it has connection with other problems, such as the behavior of the classical Radon transform (see Nagel, Riviere and Wainger [27], Marstrand [22], Falconer [7], Strichartz [45], and Oberlin and Stein [29]), and the behavior of solutions of the wave equation, and more generally other hyperbolic equations (see Stein [39], Greenleaf [10], Ruiz [31], and Sogge [34]).

The starting point for intensive studies associated with averages over low dimensional sets is based on an earlier bound of Stein [39] from 1976 on the spherical maximal function

$$
\mathcal{M} f(x):=\sup _{t>0}\left|M_{t} f(x)\right|
$$

where $M_{t}$ are the spherical averaging operators

$$
\mathcal{M}_{t} f(x)=\int_{|y|=1} f(x-t y) d \sigma(y)
$$

and $d \sigma$ is normalized surface measure on the sphere $S^{n-1}$. Then Stein's fundamental result shows that for $n \geq 3$, the corresponding spherical maximal operator is bounded on $L^{p}\left(\mathbb{R}^{n}\right)$ for every $p>n /(n-1)$. One can use this bound to show the pointwise 
covergence $\lim _{t \rightarrow 0} M_{t} f(x)=f(x)$ a.e.. The analogous result in dimension two was later proved by J. Bougain [2].

Then one turned to deal with generalizations of $\mathcal{M}_{t}$ defined as before, i.e. the sphere is replaced by a more general smooth hypersurface $S \in \mathbb{R}^{n}$. Let $\rho \in C_{0}^{\infty}(S)$ be a smooth non-negative function with compact support. Then the associated maximal operator is defined as

$$
\mathcal{M} f(x):=\sup _{t>0}\left|\int_{S} f(x-t y) \rho(y) d \mu(y)\right|, \quad x \in \mathbb{R}^{n},
$$

where $d \mu$ denotes the surface measure on $S$. Greenleaf [10] proved that $\mathcal{M}$ is bounded on $L^{p}\left(\mathbb{R}^{n}\right)$ if $n \geq 3$ and $p>(k+1) / k$, provided $S$ has at least $k \geq 2$ non-vanishing principal curvatures and $S$ is starshaped with respect to the origin.

A fundamental open problem is to characterize the $L^{p}$ boundedness properties of the maximal operator associated to hypersurface where the Gaussian curvature at some points is allowed to vanish, with the exception of the 2 dimensional case, i.e. the case of finite type curves in $\mathbb{R}^{2}$ studied by A. Iosevich in [12].

Many authors put a lot of effort on the development of this subject and obtained partial results in high dimension. Sogge and Stein [37] showed that if the Gaussian curvature of $\mathrm{S}$ does not vanish of infinite order at any point of $\mathrm{S}$, then there exists a $p_{0}(S)<\infty$ so that the maximal function is bounded on $L^{p}, p>p_{0}(S)$. However, the exponent $p_{0}(S)$ given in that paper is in general far from being optimal. In order to find the smallest value of $p_{0}(S)$, one will put more restriction on the surfaces. It is worth mentioning that in 1992, Sogge [36] employed the local smoothing estimate to get the $L^{p}$-boundedness $p>2$ for $\mathcal{M}$, where the surface has at least one non-vanishing principal curvature everywhere in $\mathbb{R}^{n}(n \geq 2)$. The perhaps best understood class in higher dimension is the class of convex hypersurface of finite line type, and the related work in this settings included Cowling and Mauceri [4] [5], Nagel, Seeger and Wainger[28], Iosevich and Sawyer [13] [14], and Iosevich, Sawyer and Seeger [15]. In particular, Iosevich and Sawyer proved in [14] sharp $L^{p}$-estimates for maximal functions related to the surface which are given by smooth convex functions of finite line type for $p>2$. In 2010, Ikoromov, Kempe and Müller [11] discovered a connection between the $L^{p}$-boundedness $(p>\max \{h(S), 2\})$ of $\mathcal{M}$ and the height $h(S)$ of a smooth, compact hypersurface $S$ of finite type in $\mathbb{R}^{3}$ satisfying the transversality assumption (i.e. for every $x \in S$, the affine tangent plane $x+T_{x} S$ to $S$ through $x$ does not pass through the origin in $\mathbb{R}^{3}$ ) on $S$. Recently, Zimmermann in his thesis [47] proved that maximal averages over analytic hypersurfaces passing through the origin in general behave more regularly than the maximal averages over hypersurfaces satisfying the transversality condition. 
Maximal operators defined by averages over curves or surfaces with non-isotropic dilations have also been extensively considered, i.e. the dilation ty appearing in (1.2) is replaced by $\delta_{t}(y)=\left(t^{a_{1}} y_{1}, t^{a_{2}} y_{2}, \cdots, t^{a_{n}} y_{n}\right)$, where $a_{j}>0, j=1,2, \cdots, n$. In 1970, in the study of a problem related to Poisson integrals for symmetric spaces, Stein raised the question as to when the operator $\mathcal{M}_{\gamma}$ defined by

$$
\mathcal{M}_{\gamma} f(x)=\sup _{h>0} \frac{1}{h} \int_{0}^{h}|f(x-\gamma(t))| d t,
$$

where $\gamma(t)=\left(A_{1} t^{a_{1}}, A_{2} t^{a_{2}}, \cdots, A_{n} t^{a_{n}}\right)$ and $A_{1}, A_{2}, \cdots, A_{n}$ are real, $a_{i}>0$, is bounded on $L^{p}\left(\mathbb{R}^{n}\right)$. Nagel, Riviere and Wainger [27] showed that the $L^{p}$-boundedness of $\mathcal{M}$ holds for $p>1$ for the special case $\gamma(t)=\left(t, t^{2}\right)$ in $\mathbb{R}^{2}$ and Stein [40] for homogeneous curves in $\mathbb{R}^{n}$. More general maximal operators involving two-parameter dilations related to homogeneous curves have studied by Marletta and Ricci [21]. For maximal functions $\mathcal{M}$ associated with nonisotropic dilations in higher dimensions, one can see the work by Greenleaf [10], Sogge and Stein [37], Iosevich and Sawyer [14], Ikoromov, Kempe and Müller [11], Zimmermann [47].

\section{$1.2 \quad$ Local smoothing estimates}

Let $S \subset \mathbb{R}^{2}$ be a smooth curve whose curvature does not vanish anywhere, and let $d \sigma$ be the induced Lebesgue measure. The averaging operators are defined by

$$
A_{t} f(x):=\int_{S} f(x-t y) d \sigma(y), \quad f \in C_{0}^{\infty}\left(\mathbb{R}^{2}\right),
$$

the associated maximal operator is given by

$$
\mathcal{M} f(x):=\sup _{t>0}\left|A_{t} f(x)\right|, \quad x \in \mathbb{R}^{n} .
$$

Then $\|\mathcal{M} f\|_{L^{p}\left(\mathbb{R}^{2}\right)} \leq\|f\|_{L^{p}\left(\mathbb{R}^{2}\right)}$, for $2<p<\infty$. This is a theorem of Bourgain [2]. An alternative approach to the result for circular maximal operators has been devised by Mockenhaupt, Seeger and Sogge [26]. Since the averaging operators $A_{t}$ can be expressed as Fourier integral operators, the authors of [25] develop a more sophisticated theory of local smoothing estimates of Fourier integral operators satisfying the so-called cinematic curvature condition so that they can improve many estimates for maximal operators. In particular, they are able to prove the variable coefficient version of the circular maximal theorem, which includes estimates for averages over geodesic circles. These general local smoothing estimates for Fourier integral operators have also other 
applications, one of which is to deal with a special class which contains the solution operators for the Cauchy problem associated to variable coefficient wave equations.

The phrase "local smoothing" was first used for certain types of estimates for dispersive equations which go back to Kato [16], Sjölin [33], and Vega [46]. Here we only show one of the deep results obtained by Mockenhaupt, Seeger and Sogge in order to easily understand the phrase "local smoothing" from estimates for the following Cauchy problem for the wave equation

$$
\begin{gathered}
\left((\partial / \partial t)^{2}-\Delta\right) u=0, \\
\left.u\right|_{t=0}=f,\left.\quad(\partial / \partial t) u\right|_{t=0}=g, \quad f, g \in C_{0}^{\infty}\left(\mathbb{R}^{2}\right),
\end{gathered}
$$

where the space variable $x$ is in $\mathbb{R}^{2}$ and $\Delta$ is the Laplace operator. Let $\alpha(p)=1 / p$, if $4 \leq p<\infty$, and $\alpha(p)=1 / 2-1 / p$, if $2<p<4$. Assume $2<p<\infty$, then for any compact time interval $I$,

$$
\left(\iint_{\mathbb{R}^{2} \times I}|u(x, t)|^{p} d x d t\right)^{1 / p} \leq C\left(\|f\|_{L_{\gamma}^{p}}+\|g\|_{L_{\gamma-1}^{p}}\right), \text { if } \gamma>1 / 2-1 / p-\alpha(p) / 2,
$$

where $C$ only depends on $I, p$, and $L_{\gamma}^{p}$ denotes the usual Sobolev space of order $\gamma$.

For fixed $t \neq 0$ the analogue of (1.3) holds if and only if $\gamma>1 / 2-1 / p$ (see Miyachi [24], Peral [30] and Seeger [32]). Thus this result says that there is "local smoothing" of order $\alpha(p) / 2$.

Furthermore, we show some main ideas of the proof of the $L^{p}$-boundedness of the circular maximal operator in [26]. However, in $\mathbb{R}^{2}$ one cannot use the argument from [10], since the square functions that control the maximal operator are not bounded on any $L^{p}$ space. To avoid this phenomenon, Mockenhaupt, Seeger and Sogge in [26] make use of the Little-Paley theory and the Sobolev embedding theorem in order to show that it is sufficient to prove certain estimates involving $L^{p}$-averages of derivatives of $A_{t}$ with respect to the $t$ variable. It is not difficult to see that there can never be $L^{p} \rightarrow L^{p}$ local smoothing when $p \leq 2$ or $p=\infty$. From the above arguments, local smoothing estimates for the corresponding Fourier integral operator gives $L^{4}$-estimates with an extra bit of regularity, together with the easy endpoint estimates on $L^{2}$ and $L^{\infty}$, finally the M. Riesz interpolation theorem implies the $L^{p}$-boundedness $(p>2)$ of corresponding maximal operators. Now the challenge is how to get the extra bit of regularity in the $L^{4}$-estimate for the corresponding Fourier integral operator. Their proof relies greatly on the fact that a natural cone attached to the Fourier integral operator associated to the wave equation is the light cone $\{(\xi,|\xi|)\}$, whose level curves $|\xi|=$ const have non-vanishing curvature. Note that this is equivalent to saying that the cinematic curvature of the 
Fourier integral operator ( i.e. the curvature of the curve $\{\xi:|\xi|=1\}$ ) is equal to 1. Mockenhaupt, Seeger and Sogge employ dyadic decompositions and angular decompositions to give orthogonality arguments and reduce the problem to Kakeya type maximal functions.

A very natural question is to ask how the $L^{4}$-estimate for a Fourier integral operator depends on the cinematic curvature. In this thesis, we show that a class of Fourier integral operators which do not satisfy the cinematic curvature condition uniformly, still satisfy a local smoothing estimate. Just when we finished our work and searched for background materials on "cinematic curvature" online, it came to our attention that in 2000, Kung already obtained some results for a related problem in his thesis [17] which has not been published until now and only show the results in [18] which we had not been aware of. We are thankful to Kung who provided us with his thesis soon after we wrote an Email to him. Through reading his thisis we know that the basic structure of both Kung's and our approach might appear similar, since both strategies rely on papers [26] and [25] by Mockenhaupt, Seeger and Sogge. Nevertheless, our approach differs from [17], since we made use of a different angular decomposition. In this way, we obtain stronger $L^{4}$ estimates for a class of Fourier integral operators than in [17], which can be applied on establishing the $L^{p}$-boundedness of the maximal operator associated with dilations $\left(x_{1}, x_{2}\right) \rightarrow\left(t x_{1}, t^{d} x_{2}\right)$ of the curve $x \rightarrow\left(x, x^{d}(1+\phi(x))\right)$, where $\phi(x)=\mathcal{O}\left(x^{m}\right), m \geq 1$ and $d \geq 2$. In a model case, the associated cone for the corresponding Fourier integral operator $\mathcal{F}_{\lambda}^{\delta}$ which is localized to frequencies $|\xi| \approx \lambda$ is of the form $\{(\xi, \delta q(\xi))\}$, where $\delta>0$ is very small and $q(\xi)$ is homogeneous of degree one, smooth on the support of the symbol of $\mathcal{F}_{\lambda}^{\delta}$. Comparing the light cone, we observe that the level curves of the cone $\{(\xi, \delta q(\xi))\}$ become flatter and the cinematic curvature of $\mathcal{F}_{\lambda}^{\delta}$ is only greater than or equal to $\delta$. In [18], Kung got that $\left\|\mathcal{F}_{\lambda}^{\delta}\right\|_{L^{4} \rightarrow L^{4}} \leq \lambda^{1 / 8+\epsilon_{1}} \delta^{-1 / 2}$. However, in this thesis we obtain the better estimate that $\left\|\mathcal{F}_{\lambda}^{\delta}\right\|_{L^{4} \rightarrow L^{4}} \leq \lambda^{1 / 8+\epsilon_{1}} \delta^{-\left(3 / 8+\epsilon_{2}-\epsilon_{1}\right)}$ ( see Chapter 3 of this thesis ).

Moreover, in [18], Kung extends his estimate for the operator $\mathcal{F}_{\lambda}^{\delta}$ to more general Fourier integral operators which correspond the cones $\{(\xi, q(x, t, \xi))\}$, where for all $x$, $t$, the curvature of the curve $\{\xi: q(x, t, \xi)=1\}$ is greater than or equal to $\delta$. In view of an application to our problems, we extend our estimate for the operator $\mathcal{F}_{\lambda}^{\delta}$ to a class of Fourier integral operators which correspond the cones $\{(\xi, \delta q(t, \xi, \delta))\}$, where $q(t, \xi, \delta)$ is homogeneous of degree one in $\xi$, smooth on the support of the symbol of the corresponding Fourier integral operator ( see Chapter 4 of this thesis ). However, it is still open which exponent of $\delta$ is optimal. 


\subsection{Organization of the thesis}

In our settings, the averaging operator is always written as a Fourier integral operator, then we turn to prove $L^{p}$-boundedness for the Fourier integral operator. In Chapter 2, first, we give an overview of the theory of Fourier integral operators and a concrete description of the so-called cinematic curvature condition. Furthermore, we establish $L^{p}$-boundedness of the maximal operator related to the curve $x \rightarrow\left(x, x^{2}(1+\phi(x))\right)$, where $\phi(x)=\mathcal{O}\left(x^{m}\right)$ and $m \geq 1$, with associated dilations $\left(x_{1}, x_{2}\right) \rightarrow\left(t x_{1}, t^{2} x_{2}\right)$. After a scaling argument, application of the method of stationary phase and LittlewoodPaley theory, we observe that the phase function of the corresponding Fourier integral operator will not satisfy the cinematic curvature condition uniformly, which causes major difficulties to apply the local smoothing estimate directly to our Fourier integral operator. We separate the problem into two parts, depending on a dyadic decomposition for the frequency variables of the corresponding Fourier integral operator, i.e. low frequency and high frequency. For low frequency, we mainly use the better endpoint regularity estimates, the M. Riesz interpolation theorem to get the desired result. For high frequency, we develop a new method based on the main idea of the local smoothing estimate from [25] to get the key $L^{4}$-boundedness of the corresponding Fourier integral operator $\tilde{\mathcal{F}}_{\lambda}^{\delta}$. Later, we generalize the result to curves of finite type $d(d \geq 2)$ and associated dilations $\left(x_{1}, x_{2}\right) \rightarrow\left(t x_{1}, t^{d} x_{2}\right)$. These results answer the question which was unsolved until recently as to how to characterize the range of all $p$ for which maximal functions associated with non-isotropic dilations of non-homogeneous curves of finite type in the plane is bounded.

Chapter 3 gives a detailed proof of the $L^{4}$-estimate of the model operator, which is translation-invariant and can approximate the Fourier integral operator $\tilde{\mathcal{F}}_{\lambda}^{\delta}$.

The proof of the $L^{4}$-estimate of the Fourier integral operator $\tilde{\mathcal{F}}_{\lambda}^{\delta}$ will be given in Chapter 4 .

In Chapter 5, employing some arguments used and some results obtained in the plane, we also establish $L^{p}$-estimates for the maximal function related to the hypersurface $\left(x_{1}, x_{2}\right) \rightarrow\left(x_{1}, x_{2}, x_{2}^{d}\left(1+\mathcal{O}\left(x_{2}^{m}\right)\right)\right)$ with associated dilations $\delta_{t}(x)=\left(t^{a} x_{1}, t x_{2}, t^{d} x_{3}\right)$ for an arbitrary real number $a>0$. These results could not be covered by the theorems about maximal functions associated with nonisotropic dilations of hypersurfaces in $\mathbb{R}^{3}$ from Greenleaf [10], Iosevich-Sawyer [14], Ikromov-Kempe-Müller [11] and Zimmermann [47]. Moreover, by an alternative approach, we also get $L^{p}$-estimates for some classes of maximal functions in $\mathbb{R}^{3}$ proved in [10], [14], [11] and [47].

Conventions: Throughout this thesis, we shall use the well known notation $A \ll B$, 
which means if there is a sufficiently large constant $G$, which does not depend on the relevant parameters arising in the context in which the quantities $A$ and $B$ appear, such that $G A \leq B$. We write $A \approx B$, and mean that $A$ and $B$ are comparable. We write $A \lesssim B$ if $A \ll B$ or $A \approx B$. $A \wedge B$ means if $A \leq B$, then $A \wedge B=A$; if $A \geq B$, then $A \wedge B=B$. 


\section{Chapter 2}

Maximal functions associated with nonisotropic dilations

of curves in the plane 
Chapter 2. Maximal functions associated with nonisotropic dilations

\subsection{Statement of the main results}

In this chapter, we will show some $L^{p}$-estimates for maximal functions associated with nonisotropic dilations of curves in the plane.

The maximal operator along curves in $\mathbb{R}^{2}$ is defined by

$$
\mathcal{M} f(x)=\sup _{t>0}\left|f * \mu_{t}(x)\right|
$$

where $\mu$ is a arc length measure supported on the curve and $\mu_{t}$ is the same measure dilated by $t>0$ and appropriately normalized.

Two different situations will arise. (1) A maximal operator of the first type: the curve $x_{2}=\gamma\left(x_{1}\right)$ is homogeneous under the given dilations ( $\operatorname{such}$ as $\gamma\left(x_{1}\right)=x_{1}^{d}, d>0, d \neq 1$ ). Basically one obtains the same operator by restricting the supremum to $t=2^{j}$. Under appropriate assumptions on the curve, one can prove that $\mathcal{M}$ is bounded on $L^{p}$ for $p>1$. The exact results and proofs can be found in page 472-492 of [41]. (2) A maximal operator of the second type: the curve $x_{2}=\gamma\left(x_{1}\right)$ is not homogeneous under the given dilations. Then the various $\mu_{t}$ are supported on different curves and the problem becomes much more complicated. We have the following subcases:

(2a) if the dilations are isotropic and the Gaussian curvature of the curve does not vanish, then $\mathcal{M}$ is bounded on $L^{p}$ for $p>2$, see [2] and [25]. The range of $p$ must be further restricted if the curvature allows to vanish at some point [12] unless one introduces a damping factor [19];

(2b) if the dilations are non-isotropic and the curve with non-vanishing Gaussian curvature away from the origin is homogeneous, Marletta and Ricci [20] combine Bourgain's theorem with a Littlewood-Paley decomposition to show that $\mathcal{M}$ is bounded on $L^{p}$ if and only if $p>2$;

A natural question to ask is whether there exists some positive constant $p_{0}>1$ such that $\mathcal{M}$ is bounded on $L^{p}$ for $p>p_{0}$ in the setting (2c) if the dilations are nonisotropic and the curve $x_{2}=\gamma\left(x_{1}\right)$ is non-homogeneous and of finite type (such as $\gamma\left(x_{1}\right)=x_{1}^{d}\left(1+x_{1}^{m}+\mathcal{O}\left(x_{1}^{m+1}\right)\right), d \geq 2$ and $\left.m \geq 1\right)$ ? This chapter will give the answers.

Now we list our main results.

Let $\phi \in C^{\infty}(I, \mathbb{R})$, where $I$ is a bounded interval containing the origin, and

$$
\phi(0) \neq 0 ; \phi^{(j)}(0)=0, j=1,2, \cdots, m-1 ; \phi^{(m)}(0) \neq 0(m \geq 1) .
$$

Theorem 2.1.1. Define the maximal operator

$$
\mathcal{M} f(y):=\sup _{t>0}\left|\int_{\mathbb{R}} f\left(y_{1}-t x, y_{2}-t^{2} x^{2} \phi(x)\right) \eta(x) d x\right|,
$$


where $\eta(x)$ is supported in a sufficiently small neighborhood of the origin. If $\phi$ satisfies (2.1), then for $p>2$, there exists a constant $C_{p}$ such that the following inequality holds true:

$$
\|\mathcal{M} f\|_{L^{p}} \leq C_{p}\|f\|_{L^{p}}, \quad f \in C_{0}^{\infty}\left(\mathbb{R}^{2}\right)
$$

Remark 2.1.1. If $m \rightarrow \infty$, then we obtain the maximal operator of the first type described above which is bounded on $L^{p}$ for all $p>1$.

In fact, the above theorem is sharp if $1 \leq m<\infty$, which follow from the following proposition.

Proposition 2.1.2. If $1 \leq m<\infty$, then $p>2$ is a necessary condition for the maximal inequality (2.3).

Proof. Let $f(x)=\frac{\chi_{[-1 / 2,1 / 2]^{2}}(x)}{|x| \log \frac{1}{|x|}}$. It's clear that $f \in L^{p}\left(\mathbb{R}^{2}\right)$ if $p \leq 2$.

Now we take $\epsilon<\frac{1}{100}$ such that $[\epsilon / 2,4 \epsilon] \subset \operatorname{supp} \eta$, then fix it. We may assume that $\phi(0)>0, \phi^{(j)}(0)=0, j=1,2, \cdots, m-1$, and $\phi^{(m)}(0)>0$. Let $y_{1} \in[1 / 8,1 / 4]$ and $y_{1}^{2} \phi(\epsilon) \leq y_{2} \leq y_{1}^{2} \phi(2 \epsilon)$. By the inverse function theorem, we can set $a:=a(y)=$ $\phi^{-1}\left(y_{2} / y_{1}^{2}\right)$, then $\epsilon \leq a \leq 2 \epsilon$ (otherwise, if we assume $\phi(0)>0$ and $\phi^{(m)}(0)<0$, since $\phi^{\prime}(x)=\frac{\phi^{(m)}(0)}{(m-1) !} x^{m-1}+\mathcal{O}\left(x^{m}\right)$, then $\phi(x)$ is strictly decreasing in the interval $[\epsilon / 2,4 \epsilon]$. In this case, we take $\left.y_{1}^{2} \phi(2 \epsilon) \leq y_{2} \leq y_{1}^{2} \phi(\epsilon)\right)$. We choose $\epsilon_{1}>0$ such that $\epsilon_{1} \ll \epsilon$, then $\left\{x:|x-a|<\epsilon_{1}\right\} \subset \operatorname{supp} \eta$. Now we have

$$
\begin{aligned}
M f(y) & =\sup _{t>0}\left|\int f\left(y_{1}-t x, y_{2}-t^{2} x^{2} \phi(x)\right) \eta(x) d x\right| \\
& =\sup _{t>0} \int \frac{\chi_{[-1 / 2,1 / 2]^{2}}\left(y_{1}-t x, y_{2}-t^{2} x^{2} \phi(x)\right)}{\left|\left(y_{1}-t x, y_{2}-t^{2} x^{2} \phi(x)\right)\right| \log \frac{1}{\left|\left(y_{1}-t x, y_{2}-t^{2} x^{2} \phi(x)\right)\right|}} \eta(x) d x \\
& \geq \int_{\left\{x:|x-a|<\epsilon_{1}\right\}} \frac{\chi_{[-1 / 2,1 / 2]^{2}}\left(y_{1}-t_{0} x, y_{2}-t_{0}^{2} x^{2} \phi(x)\right)}{\left|\left(y_{1}-t_{0} x, y_{2}-t_{0}^{2} x^{2} \phi(x)\right)\right| \log \frac{1}{\left|\left(y_{1}-t_{0} x, y_{2}-t_{0}^{2} x^{2} \phi(x)\right)\right|}} d x
\end{aligned}
$$

where $t_{0}=y_{1} / a$. We also observe that $\frac{1}{16 \epsilon} \leq t_{0} \leq \frac{1}{4 \epsilon}$.

If $|x-a|<\epsilon_{1}$, then $x$ can be written as $x=a+z=\frac{y_{1}}{t_{0}}+z,|z|<\epsilon_{1}$, which gives $\left|y_{1}-t_{0} x\right|=t_{0}\left|x-\frac{y_{1}}{t_{0}}\right|=t_{0}|z| \leq \frac{\epsilon_{1}}{4 \epsilon}<1 / 4$, and $\left.\mid y_{2}-t_{0}^{2} x^{2} \phi(x)\right) \mid$ is equal to

$$
\begin{aligned}
& \left|y_{2}-\frac{y_{1}^{2}}{a^{2}}(a+z)^{2}\left(\phi(a)+\phi^{\prime}(a) z+\mathcal{O}\left(z^{2}\right)\right)\right| \\
& =\left|y_{2}-\frac{y_{1}^{2}}{a^{2}} a^{2} \phi(a)-\frac{y_{1}^{2}}{a^{2}}\left(2 a z+z^{2}\right) \phi(a+z)-\frac{y_{1}^{2}}{a^{2}} a^{2}\left(\phi^{\prime}(a) z+\mathcal{O}\left(z^{2}\right)\right)\right| \\
& =\left|\frac{y_{1}^{2}}{a^{2}}\left(2 a z+z^{2}\right) \phi(a+z)+y_{1}^{2}\left(\phi^{\prime}(a) z+\mathcal{O}\left(z^{2}\right)\right)\right| \\
& =t_{0}|z|\left|y_{1}\left(2+\frac{z}{a}\right) \phi(a+z)+a y_{1}\left(\phi^{\prime}(a)+\mathcal{O}(z)\right)\right|
\end{aligned}
$$




$$
\leq \frac{\epsilon_{1}}{4 \epsilon}\left[\frac{1}{4}\left(2+\frac{\epsilon_{1}}{\epsilon}\right) \phi(4 \epsilon)+\frac{\epsilon}{2}\left(\phi(a)+\mathcal{O}\left(\epsilon_{1}\right)\right)\right] t_{0}|z|<1 / 4
$$

Set $g(z)=y_{1}\left(2+\frac{z}{a}\right) \phi(a+z)+a y_{1}\left(\phi^{\prime}(a)+\mathcal{O}(z)\right)$ which is bounded from above by, say $\tilde{C}$. From the above argument, we have $\left(y_{1}-t_{0} x, y_{2}-t_{0}^{2} x^{2} \phi(x)\right) \in[-1 / 2,1 / 2]$. Then making a change of variables, we have

$$
\begin{aligned}
M f(y) & \geq \int_{\left\{x:|x-a|<\epsilon_{1}\right\}} \frac{d x}{\left|\left(y_{1}-t_{0} x, y_{2}-t_{0}^{2} x^{2} \phi(x)\right)\right| \log \frac{1}{\left|\left(y_{1}-t_{0} x, y_{2}-t_{0}^{2} x^{2} \phi(x)\right)\right|}} \\
& \geq \int_{0}^{\epsilon_{1}} \frac{d z}{t_{0} z|(1, g(z))| \log \frac{1}{t_{0} z|(1, g(z))|}} .
\end{aligned}
$$

Let $C=\sqrt{1+\tilde{C}^{2}}$. It is a fact that $\frac{1}{x \log \frac{1}{x}}$ decrease monotonically in the neighborhood of the origin. Together with the above estimates, we will have

$$
\frac{1}{t_{0} z|(1, g(z))| \log \frac{1}{t_{0} z|(1, g(z))|}} \geq \frac{1}{C t_{0} z \log \frac{1}{C t_{0} z}}
$$

Finally, for the fixed $\epsilon$ and $\epsilon_{1} \ll \epsilon$, we have

$$
M f(y) \geq \int_{0}^{\epsilon_{1}} \frac{d z}{C t_{0} z \log \frac{1}{C t_{0} z}} \geq \frac{4 \epsilon}{C} \int_{0}^{C \epsilon_{1} /(16 \epsilon)} \frac{d z}{z \log \frac{1}{z}}=\infty
$$

Next we will generalize Theorem 2.1.1 to the curve of finite type $d(\geq 2)$ associated with the function $\phi$ defined by (2.1).

Theorem 2.1.3. Define the maximal operator

$$
\mathcal{M} f(y):=\sup _{t>0}\left|\int_{\mathbb{R}} f\left(y_{1}-t x, y_{2}-t^{d} x^{d} \phi(x)\right) \eta(x) d x\right|
$$

where $\eta(x)$ is supported in a sufficiently small neighborhood of the origin. If $\phi$ satisfies (2.1) and $d \geq 2$, then for $p>2$, there exists a constant $C_{p}$ such that

$$
\|\mathcal{M} f\|_{L^{p}} \leq C_{p}\|f\|_{L^{p}}, \quad f \in C_{0}^{\infty}\left(\mathbb{R}^{2}\right)
$$

Remark 2.1.2. If $m \rightarrow \infty$, then $\mathcal{M}$ coincides with the maximal operator of the second type described above. 


\subsection{Background on Fourier integral operators and auxiliary results}

\subsubsection{Local smoothing of Fourier integral operators}

In our settings, the averaging operator is always expressed as a Fourier integral operator, then we turn to prove the $L^{p}$ boundedness for these operators. So here we will make a brief introduction to local smoothing of Fourier integral operators in [25].

We consider a class of Fourier integral operators $I^{\nu}\left(\mathbb{R}^{n+1}, \mathbb{R}^{n} ; \Lambda\right)$, which is determined by the properties of its canonical relation $\Lambda$, which is a conic Lagrangian in $T^{*} Y \backslash 0$ to $T^{*} Z \backslash 0$ with respect to the symplectic form $d \zeta \wedge d z-d \eta \wedge d y$, and closed in $T^{*} Z \backslash 0 \times T^{*} Y \backslash 0$. In fact, these assumptions imply that $\Lambda \subset T^{*} Z \backslash 0 \times T^{*} Y \backslash 0$ is a conic (immersed) submanifold of dimension $2 n+1$.

To guarantee local regularity properties of operators $\mathcal{F} \in I^{\nu}\left(\mathbb{R}^{n+1}, \mathbb{R}^{n} ; \Lambda\right)$, we shall impose conditions on $\Lambda$ which are based on the properties of the following three projections

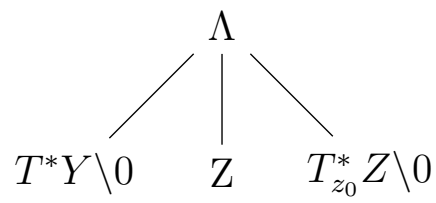

We assume that $\Pi_{X}$ is the projection of $\Lambda$ onto $\mathrm{X}, \mathrm{X}=T_{z_{0}}^{*} Z \backslash 0$, Z, or $T^{*} Y \backslash 0$. The condition has two parts:

(1) non-degeneracy condition:

$$
\begin{aligned}
& \operatorname{rank} d \Pi_{T^{*} Y} \equiv 2 n, \\
& \text { rank } d \Pi_{Z} \equiv n+1 ;
\end{aligned}
$$

analogue of the Carleson-Sjölin condition for nonhomogeneous phase (for every $z_{0} \in Z$, $\Gamma_{z_{0}}:=\Pi_{T_{z_{0}}^{*} Z}(\Lambda)$ has $n$ non-vanishing principal curvature);

(2) cone condition: for every $z_{0} \in Z, \Gamma_{z_{0}}$ is a smooth conic $n$-dimensional hypersurface, $n-1$ principal curvatures do not vanish. A Fourier integral operator which satisfies (2.6), (2.7) and the cone condition is said to satisfy the "cinematic curvature" condition. The exact description of this condition can be found in [35]. Here we like to see how the condition can be reformulated if we use local coordinates. The non-degeneracy 
condition implies that near a given point $\left(z_{0}, \zeta_{0}, y_{0}, \eta_{0}\right) \in \Lambda$, local coordinates can be chosen so that $\Lambda \ni(z, \zeta, y, \eta) \rightarrow(z, \eta)$ has bijective differential and there must be a phase function $\varphi(z, \eta)$ so that $\Lambda$ takes the form

$$
\left\{\left(z, \varphi_{z}^{\prime}(z, \eta), \varphi_{\eta}^{\prime}(z, \eta), \eta\right): \eta \in \mathbb{R}^{n} \backslash 0 \text { in a conic neighborhood of } \eta_{0}\right\}
$$

In this case, the condition (2.6) becomes

$$
\operatorname{rank} \varphi_{z, \eta}^{\prime \prime} \equiv n
$$

which means if we fix $z_{0}$, then,

$$
\Gamma_{z_{0}}=\left\{\varphi_{z}^{\prime}\left(z_{0}, \eta\right): \eta \in \mathbb{R}^{n} \backslash 0 \text { in a conic neighborhood of } \eta_{0}\right\} \subset T_{z_{0}}^{*} Z \backslash 0
$$

must be a smooth conic submanifold of dimension $n$. Then if $\Gamma_{z_{0}} \ni \zeta=\varphi_{z}^{\prime}\left(z_{0}, \eta\right)$ and $\theta \in S^{n}$ is normal to $\Gamma_{z_{0}}$ at $\zeta$, it follows that $\pm \theta$ are the unique directions for which $\nabla_{\eta}\left\langle\varphi_{z}^{\prime}\left(z_{0}, \eta\right), \theta\right\rangle=0$. The cone condition (2) is just that

$$
\operatorname{rank}\left(\frac{\partial^{2}}{\partial \eta_{j} \partial \eta_{k}}\right)\left\langle\varphi_{z}^{\prime}\left(z_{0}, \eta\right), \theta\right\rangle=n-1 \text { if } \eta, \theta \text { are obtained from the above. }
$$

Suppose $\mathcal{F} \in I^{\mu-1 / 4}(Z, Y ; \Lambda)$, where $\Lambda$ satisfies (2.6), (2.7) and (2.8), $\mathcal{F} f$ can be written as a finite sum of the form

$$
\int_{\mathbb{R}^{n}} e^{i \varphi(z, \eta)} a(z, \eta) \widehat{f}(\eta) d \eta, \quad f \in C_{0}^{\infty}\left(\mathbb{R}^{n}\right),
$$

where the phase function $\varphi$ satisfyies (2.9) and (2.10) and the symbol $a$ of order $\mu$ has small conic support in $\mathbb{R}^{n+1} \times \mathbb{R}^{n}$, which means that $a$ vanishes for all $z$ outside a small compact set and for all $\eta=\left(\eta_{1}, \eta^{\prime}\right)$ outside a narrow cone $\left\{\eta:\left|\eta^{\prime}\right| \leq \varepsilon \eta_{1}\right\}$.

In this thesis, we always consider the case $n=2$ and $z=(x, t) \in \mathbb{R}^{2} \times \mathbb{R}$. We fix $\beta \in C_{0}^{\infty}(\mathbb{R})$ supported in $[1 / 2,2]$ and set $a_{\lambda}(x, t, \eta)=\lambda^{-\mu} \beta(|\eta| / \lambda) a(x, t, \eta)$ for fixed $\lambda>1$. Then $a_{\lambda}$ is a symbol of order zero and satisfies the usual symbol estimates uniformly in $\lambda$. Mockenhaupt, Seeger and Sogge show that the dyadic estimate of the Fourier integral operator $\mathcal{F}$ is as following.

Theorem 2.2.1. [26]

$$
\left(\int_{1 / 2}^{4} \int_{\mathbb{R}^{2}}\left|\int_{\mathbb{R}^{2}} e^{i \varphi(x, t, \eta)} a_{\lambda}(x, t, \eta) \widehat{f}(\eta) d \eta\right|^{p} d x d t\right)^{1 / p} \leq C_{p} \lambda^{1 / 2-1 / p-\epsilon(p)},
$$

where $\epsilon(p)=\frac{1}{2 p}$, if $4 \leq p<\infty ; \epsilon(p)=\frac{1}{2}\left(\frac{1}{2}-\frac{1}{p}\right)$, if $2<p \leq 4$. 


\subsubsection{Auxiliary results}

We will often use the following method of stationary phase.

Lemma 2.2.2. (Theorem 1.2.1 in [35]) Let $S$ be a smooth hypersurface in $\mathbb{R}^{n}$ with non-vanishing Gaussian curvature and $d \mu$ be the Lebesgue measure on $S$. Then,

$$
|\widehat{d \mu}(\xi)| \leq C(1+|\xi|)^{-\frac{n-1}{2}} .
$$

Moreover, suppose that $\Gamma \subset \mathbb{R}^{n} \backslash 0$ is the cone consisting of all $\xi$ which are normal to $S$ at some point $x \in S$ belonging to a fixed relatively compact neighborhood $\mathcal{N}$ of supp $d \mu$. Then

$$
\begin{aligned}
\left|\left(\frac{\partial}{\partial \xi}\right)^{\alpha} \widehat{d \mu}(\xi)\right| & =\mathcal{O}(1+|\xi|)^{-N} \text { for all } N \in \mathbb{N}, \text { if } \xi \notin \Gamma, \\
\widehat{d \mu}(\xi) & =\sum e^{-i\left\langle x_{j}, \xi\right\rangle} a_{j}(\xi) \quad \text { if } \xi \in \Gamma
\end{aligned}
$$

where the finite sum is taken over all $x_{j} \in \mathcal{N}$ having $\xi$ as the normal and

$$
\left|\left(\frac{\partial}{\partial \xi}\right)^{\alpha} a_{j}(\xi)\right| \leq C_{\alpha}(1+|\xi|)^{-\frac{n-1}{2}-|\alpha|} .
$$

We also use the following well-known estimate.

Lemma 2.2.3. (Theorem 2.4.2 in [35]) Suppose that $F$ is $C^{1}(\mathbb{R})$. Then if $p>1$ and $1 / p+1 / p^{\prime}=1$,

$$
\sup _{\lambda}|F(\lambda)|^{p} \leq|F(0)|^{p}+p\left(\int|F(\lambda)|^{p} d \lambda\right)^{1 / p^{\prime}}\left(\int\left|F^{\prime}(\lambda)\right|^{p} d \lambda\right)^{1 / p} .
$$

Next, we will introduce the definition of the nonisotropic Littlewood-Paley operator associated with a parameter and show a related lemma.

Let $\beta \in C_{0}^{\infty}(\mathbb{R})$ be non-negative and supp $\beta \subset\left[1 / \sqrt{c_{0}}, \sqrt{c_{0}}\right]$, where $c_{0}>1$ is a real number. For a fixed integer $j>0$, real numbers $a_{1}, a_{2}>0$ and $\ell \in \mathbb{Z}$, we define the non-isotropic Littlewood-Paley operator with parameter $j$ as

$$
\widehat{\Delta_{\ell}^{j}} f(\xi)=\beta\left(2^{-j}\left|\delta_{2^{\ell}} \xi\right|\right) \hat{f}(\xi),
$$

where $\delta_{2^{\ell}} \xi=\left(2^{\ell a_{1}} \xi_{1}, 2^{\ell a_{2}} \xi_{2}\right)$.

Lemma 2.2.4. Let

$$
g_{j}(f)=\left(\sum_{\ell \in \mathbb{Z}}\left|\Delta_{\ell}^{j} f\right|^{2}\right)^{1 / 2}
$$

then the following holds true:

$$
\left\|g_{j}(f)\right\|_{L^{p}\left(\mathbb{R}^{2}\right)} \leq C_{p}\|f\|_{L^{p}\left(\mathbb{R}^{2}\right)} .
$$

if $p \in(1, \infty)$, and $C_{p}$ only depends on $p$. 
Proof. The idea of proof is very similar to the classic one in [38]. For completeness, we give the proof here. For $a=\left(a_{1}, a_{2}\right)$ and $\alpha=\left(\alpha_{1}, \alpha_{2}\right)$, we define $(a, \alpha)=a_{1} \alpha_{1}+a_{2} \alpha_{2}$, $\xi^{\alpha}=\xi_{1}^{\alpha_{1}} \xi_{2}^{\alpha_{2}}$. Let $|\cdot|_{\delta}$ be a homogeneous norm, i.e. $\left|\delta_{r} \xi\right|_{\delta}=r|\xi|_{\delta}$ for $r>0$. Hörmander's theorem in nonisotropic case is usually stated in the following way.

Lemma 2.2.5. [6] Let $m(x) \in L^{\infty}\left(\mathbb{R}^{2}\right)$, and assume $m(x)$ is $N$ times continuously differentiable where $N>|a| / 2$; moreover, assume that

$$
\int_{R / 2 \leq|\xi|_{\delta} \leq 2 R}\left|R^{(a, \alpha)}\left(\frac{\partial}{\partial \xi}\right)^{\alpha} m(\xi)\right|^{2} \frac{d \xi}{R^{|a|}} \leq C_{\alpha}, \quad|m(\xi)| \leq C \text { a.e. }
$$

where $C$ is independent of $R$, say $C \geq 1$. Then there exists a constant $A_{p}$ such that

$$
\left\|T_{m} f\right\|_{L^{p}}=\left\|\mathcal{F}^{-1}(m \hat{f})\right\|_{L^{p}} \leq A_{p}\|f\|_{L^{p}}, \quad 1<p<\infty, \quad f \in C_{0}^{\infty}\left(\mathbb{R}^{2}\right),
$$

where $A_{p}$ depends only on a and $p$. In particular, if $m$ satisfies that

$$
\left|\left(\frac{\partial}{\partial \xi}\right)^{\alpha} m(\xi)\right| \leq C|\xi|_{\delta}^{-(a, \alpha)}
$$

for all $\xi \neq 0$, then $T_{m}$ is bounded on $L^{p}\left(\mathbb{R}^{2}\right)$.

Set

$$
R_{\ell}:=\left\{\begin{aligned}
r_{2 \ell}, & \text { if } \ell \in \mathbb{Z}, \quad \ell>0 \\
r_{|2 \ell|+1}, & \text { if } \ell \in \mathbb{Z}, \quad \ell \leq 0,
\end{aligned}\right.
$$

where $\left\{r_{k}\right\}_{k \in \mathbb{N}}$ is the Rademacher function system on $[0,1]$. Then

$$
g_{j}(f)(x)^{p} \approx \int_{0}^{1}\left|\sum_{\ell \in \mathbb{Z}} \Delta_{\ell}^{j} f(x) R_{\ell}(t)\right|^{p} d t .
$$

Since $|\cdot|_{\delta} \approx|\cdot|$ for $|\xi| \approx 1$, then it is easy to see that $\sum_{\ell \in \mathbb{Z}} \beta\left(2^{-j}\left|\delta_{2^{\ell}} \xi\right|\right) \leq C$. So we can define

$$
T_{m_{j}, t} f:=\left(\sum_{\ell \in \mathbb{Z}} R_{\ell}(t) \Delta_{\ell}^{j}\right) f
$$

then $T_{m_{j}, t}$ is a Fourier multiplier operator with multiplier

$$
m_{j, t}(\xi):=\sum_{\ell \in \mathbb{Z}} R_{\ell}(t) \beta\left(2^{-j}\left|\delta_{2^{\ell}} \xi\right|\right) .
$$


However, $\left|R_{\ell}(t)\right| \leq 1$ for all $\ell \in \mathbb{Z}$ and $\left\|T_{m_{j}, t}\right\|_{L^{p} \rightarrow L^{p}}=\left\|T_{m_{0}, t}\right\|_{L^{p} \rightarrow L^{p}}$. In fact, if $\tau_{j} f(x)=2^{2 j / p} f\left(2^{j} x\right)$, then

$$
\begin{aligned}
T_{m_{j}, t} f & =T_{2^{2 j / p} \tau_{-j} m_{0, t}} f \\
& =\mathcal{F}^{-1}\left(2^{2 j / p} \tau_{-j} m_{0, t} \hat{f}\right) \\
& =2^{2 j / p} \mathcal{F}^{-1}\left(\tau_{-j}\left(m_{0, t} \hat{f}\left(2^{j} \cdot\right)\right)\right) \\
& =2^{2 j} 2^{-2 j / p} \tau_{j} \mathcal{F}^{-1}\left(m_{0, t} \hat{f}\left(2^{j} \cdot\right)\right) \\
& =\tau_{j} \mathcal{F}^{-1}\left(m_{0, t} 2^{-2 j / p} 2^{2 j} \hat{f}\left(2^{j} \cdot\right)\right) \\
& =\tau_{j} \mathcal{F}^{-1}\left(m_{0, t} \widehat{\tau_{-j} f}\right)=\tau_{j} T_{m_{0, t}}\left(\tau_{-j} f\right),
\end{aligned}
$$

and $\left\|\tau_{j} T_{m_{0, t}} \tau_{-j}\right\|_{L^{p} \rightarrow L^{p}}=\left\|T_{m_{0, t}}\right\|_{L^{p} \rightarrow L^{p}}$ implies the desired estimate.

It thus suffices to show that $m_{0, t}$ satisfies (2.18). Since for some $\ell \in \mathbb{Z}, \beta\left(\left|\delta_{2^{\ell}} \xi\right|\right) \neq 0 \Rightarrow$ $|\xi|_{\delta} \approx 2^{-\ell}$, then

$$
\sum_{\ell \in \mathbb{Z}}\left(\frac{\partial}{\partial \xi}\right)^{\alpha} \beta\left(\left|\delta_{2^{\ell}} \xi\right|\right) \leq C_{\alpha} C|\xi|_{\delta}^{-(a, \alpha)}
$$

for arbitrary $\xi \neq 0$, which implies that $m_{0, t}$ satisfies (2.18). Then there exists a constant $A_{p}$ such that

$$
\left\|T_{m_{j, t}} f\right\|_{L^{p}} \leq A_{p}\|f\|_{L^{p}}, \text { for all } f \in L^{p}\left(\mathbb{R}^{2}\right)
$$

Furthermore, (2.19) and Fubini's theorem gives

$$
\begin{aligned}
\left\|g_{j} f\right\|_{L^{p}}^{p} & \approx \int_{\mathbb{R}^{2}}\left(\int_{0}^{1}\left|T_{m_{j, t}} f\right|^{p} d t\right) d x \\
& =\int_{0}^{1}\left\|T_{m_{j, t}} f\right\|_{L^{p}}^{p} d t \\
& \leq \int_{0}^{1} A_{p}^{p}\|f\|_{L^{p}}^{p} d t=A_{p}^{p}\|f\|_{L^{p}}^{p} .
\end{aligned}
$$

\subsection{The proof for curves with non-vanishing Gaus- sian curvature}

In this section, we will give the proof of Theorem 2.1.1. 


\subsubsection{The case when $m=1$}

In this section, $\phi \in C^{\infty}(\mathbb{R})$ satisfies the following condition

$$
\phi(0) \neq 0, \quad \phi^{\prime}(0) \neq 0 .
$$

We choose $B>0$ very small and $\tilde{\rho} \in C_{0}^{\infty}(\mathbb{R})$ such that supp $\tilde{\rho} \subset\{x: B / 2 \leq|x| \leq 2 B\}$ and $\sum_{k} \tilde{\rho}\left(2^{k} x\right)=1$ for $x \in \mathbb{R}$.

Put

$$
\begin{aligned}
A_{t} f(y): & =\int f\left(y_{1}-t x, y_{2}-t^{2} x^{2} \phi(x)\right) \eta(x) d x \\
& =\sum_{k} \int f\left(y_{1}-t x, y_{2}-t^{2} x^{2} \phi(x)\right) \tilde{\rho}\left(2^{k} x\right) \eta(x) d x=\sum_{k} A_{t}^{k} f(y),
\end{aligned}
$$

where

$$
A_{t}^{k} f(y):=\int f\left(y_{1}-t x, y_{2}-t^{2} x^{2} \phi(x)\right) \tilde{\rho}\left(2^{k} x\right) \eta(x) d x
$$

Since $\eta$ is supported in a sufficiently small neighborhood of the origin, then we only need to consider $k>0$ sufficiently large.

Considering the isometric operator on $L^{p}\left(\mathbb{R}^{2}\right)$ defined by $T_{k} f\left(x_{1}, x_{2}\right)=2^{3 k / p} f\left(2^{k} x_{1}, 2^{2 k} x_{2}\right)$, one can compute that

$$
T_{k}^{-1} A_{t}^{k} T_{k} f(y)=2^{-k} \int f\left(y_{1}-t x, y_{2}-t^{2} x^{2} \phi\left(\frac{x}{2^{k}}\right)\right) \tilde{\rho}(x) \eta\left(2^{-k} x\right) d x .
$$

Then it suffices to prove the following estimate

$$
\sum_{k} 2^{-k}\left\|\sup _{t>0}\left|\widetilde{A_{t}^{k}}\right|\right\|_{L^{p} \rightarrow L^{p}} \leq C_{p}, \text { for all } p>2,
$$

where

$$
\widetilde{A_{t}^{k}} f(y):=\int f\left(y_{1}-t x, y_{2}-t^{2} x^{2} \phi\left(\frac{x}{2^{k}}\right)\right) \tilde{\rho}(x) \eta\left(2^{-k} x\right) d x .
$$

By means of the Fourier inversion formula, we have

$$
\begin{aligned}
\widetilde{A_{t}^{k}} f(y) & =\int_{\mathbb{R}^{2}} e^{i \xi \cdot y} \int_{\mathbb{R}} e^{-i\left(t \xi_{1} x+t^{2} \xi_{2} x^{2} \phi\left(\frac{x}{2^{k}}\right)\right)} \tilde{\rho}(x) \eta\left(2^{-k} x\right) d x \hat{f}(\xi) d \xi \\
& =\int_{\mathbb{R}^{2}} e^{i \xi \cdot y} \widehat{d \mu_{k}}\left(\delta_{t} \xi\right) \hat{f}(\xi) d \xi
\end{aligned}
$$

where

$$
\widehat{d \mu_{k}}(\xi):=\int_{\mathbb{R}} e^{-i\left(\xi_{1} x+\xi_{2} x^{2} \phi\left(\frac{x}{2^{k}}\right)\right)} \tilde{\rho}(x) \eta\left(2^{-k} x\right) d x
$$


We choose a non-negative function $\beta \in C_{0}^{\infty}(\mathbb{R})$ such that supp $\beta \subset[1 / 2,2]$ and $\sum_{j \in \mathbb{Z}} \beta\left(2^{-j} r\right)=1$ for $r>0$. Define the dyadic operators

$$
\widetilde{A_{t, j}^{k}} f(y)=\int_{\mathbb{R}^{2}} e^{i \xi \cdot y} \widehat{d \mu_{k}}\left(\delta_{t} \xi\right) \beta\left(2^{-j}\left|\delta_{t} \xi\right|\right) \hat{f}(\xi) d \xi
$$

and denote by $\widetilde{\mathcal{M}_{j}^{k}}$ the corresponding maximal operator. Now we have that

$$
\sup _{t>0}\left|\widetilde{A_{t}^{k}} f(y)\right| \leq \widetilde{\mathcal{M}^{k, 0}} f(y)+\sum_{j \geq 1} \widetilde{\mathcal{M}_{j}^{k}} f(y), \text { for } y \in \mathbb{R}^{2}
$$

where

$$
\widetilde{\mathcal{M}^{k, 0}} f(y):=\sup _{t>0}\left|\sum_{j \leq 0} \widetilde{A_{t, j}^{k}} f(y)\right|
$$

We observe that $\widetilde{\mathcal{M}^{k, 0}} f(y)=\sup _{t>0}\left|f * K_{\delta_{t^{-1}}}(y)\right|$, where $K_{\delta_{t^{-1}}}(x)=t^{-3} K\left(\frac{x_{1}}{t}, \frac{x_{2}}{t^{2}}\right)$ and

$$
K(y):=\int_{\mathbb{R}^{2}} e^{i \xi \cdot y} \widehat{d \mu_{k}}(\xi) \rho(|\xi|) d \xi
$$

where $\rho \in C_{0}^{\infty}(\mathbb{R})$ is supported in $[0,1]$. Since $\phi$ satisfies $(2.21)$, supp $\tilde{\rho} \subset\{x: B / 2 \leq$ $|x| \leq 2 B\}$, then Lemma 2.2.2 implies that for a multi-index $\alpha$,

$$
\left|\left(\frac{\partial}{\partial \xi}\right)^{\alpha} \widehat{d \mu_{k}}(\xi)\right| \leq C_{B, \alpha}(1+|\xi|)^{-1 / 2} .
$$

By integration by parts, we obtain that

$$
|K(y)| \leq C_{N}(1+|y|)^{-N}
$$

Then $\widetilde{\mathcal{M}^{k, 0}} f(y) \leq C_{N}^{\prime} M f(y)$, where $M$ is the Hardy-Littlewood maximal operator defined by

$$
M f(y):=\sup _{t>0} \frac{1}{|B(y, t)|} \int_{B(y, t)} f(x) d x
$$

where $B(y, t)=\left\{x:|y-x|_{\delta}<t\right\}$ and $|x|_{\delta}=\max \left\{\left|x_{1}\right|,\left|x_{2}\right|^{1 / 2}\right\}$. For the exact description, one can see page 8-13 in [41]. So it suffices to prove that

$$
\sum_{k} 2^{-k} \sum_{j \geq 1}\left\|\widetilde{\mathcal{M}_{j}^{k}}\right\|_{L^{p} \rightarrow L^{p}} \leq C_{p}
$$

Since $\widetilde{A_{t, j}^{k}}$ is localized to frequency $\left|\delta_{t} \xi\right| \approx 2^{j}$, we will show that

$$
\left\|\widetilde{\mathcal{M}_{j}^{k}}\right\|_{L^{p} \rightarrow L^{p}} \lesssim\left\|\mathcal{M}_{j, l o c}^{k}\right\|_{L^{p} \rightarrow L^{p}}
$$


where $\mathcal{M}_{j, l o c}^{k} f(y)=\sup _{t \in[1,2]}\left|\widetilde{A_{t, j}^{k}} f(y)\right|$. In fact, for fixed $j \geq 1$ and all $\ell \in \mathbb{Z}$, let $\triangle_{\ell}^{j}$ be the non-isotropic Littlewood-Paley operator in $\mathbb{R}^{2}$ defined by $\widehat{\triangle_{\ell}^{j}} f(\xi)=$ $\tilde{\beta}\left(2^{-j}\left|\delta_{2^{\ell}} \xi\right|\right) \hat{f}(\xi)$, here $\tilde{\beta} \in C_{0}^{\infty}(\mathbb{R})$ is nonnegative and satisfies $\beta\left(\left|\delta_{t} \xi\right|\right)=\beta\left(\left|\delta_{t} \xi\right|\right) \tilde{\beta}(|\xi|)$, for any $t \in[1,2]$. Then

$$
\begin{aligned}
\widetilde{M_{j}^{k}} f(y) & =\sup _{\ell \in \mathbb{Z}} \sup _{t \in[1,2]}\left|\int_{\mathbb{R}^{2}} e^{i \xi \cdot y} \widehat{d \mu_{k}}\left(\delta_{2^{\ell} t} \xi\right) \beta\left(2^{-j}\left|\delta_{2^{\ell} t} \xi\right|\right) \tilde{\beta}\left(2^{-j}\left|\delta_{2^{\ell}} \xi\right|\right) \hat{f}(\xi) d \xi\right| \\
& \leq\left(\sum_{\ell \in \mathbb{Z}} \sup _{t \in[1,2]}\left|\int_{\mathbb{R}^{2}} e^{i \xi \cdot y} \widehat{d \mu_{k}}\left(\delta_{2^{\ell} t} \xi\right) \beta\left(2^{-j}\left|\delta_{2^{\ell}} \xi\right|\right) \widehat{\triangle_{\ell}^{j}} f(\xi) d \xi\right|^{p}\right)^{1 / p} \\
& =\left(\sum_{\ell \in \mathbb{Z}} \sup _{t \in[1,2]}\left|2^{-3 \ell} \int_{\mathbb{R}^{2}} e^{i \xi \cdot \delta_{2^{-\ell}} y} \widehat{d \mu_{k}}\left(\delta_{t} \xi\right) \beta\left(2^{-j}\left|\delta_{t} \xi\right|\right) \widehat{\triangle_{\ell}^{j}} f\left(\delta_{2^{-\ell}} \xi\right) d \xi\right|^{p}\right)^{1 / p} \\
& =\left(\sum_{\ell \in \mathbb{Z}}\left|\mathcal{M}_{j, l o c}^{k}\left(\triangle_{\ell}^{j} f \circ \delta_{2^{\ell}}\right)\left(\delta_{2^{-\ell}} y\right)\right|^{p}\right)^{1 / p} .
\end{aligned}
$$

Since $p>2$, Lemma 2.2.4 implies that

$$
\begin{aligned}
\left\|\widetilde{M_{j}^{k}} f\right\|_{L^{p}}^{p} & \leq \sum_{\ell \in \mathbb{Z}} \int_{\mathbb{R}^{2}}\left|\mathcal{M}_{j, l o c}^{k}\left(\triangle_{\ell}^{j} f \circ \delta_{2^{\ell}}\right)\left(\delta_{2^{-\ell}} y\right)\right|^{p} d y \\
& =\sum_{\ell \in \mathbb{Z}} 2^{3 \ell}\left\|\mathcal{M}_{j, l o c}^{k}\left(\triangle_{\ell}^{j} f \circ \delta_{2^{\ell}}\right)\right\|_{L^{p}}^{p} \\
& \leq\left\|\mathcal{M}_{j, l o c}^{k}\right\|_{L^{p} \rightarrow L^{p}}^{p} \sum_{\ell \in \mathbb{Z}} \int_{\mathbb{R}^{2}}\left|\triangle_{\ell}^{j} f(y)\right|^{p} d y \\
& \leq\left\|\mathcal{M}_{j, l o c}^{k}\right\|_{L^{p} \rightarrow L^{p}}^{p}\left\|\left(\sum_{\ell \in \mathbb{Z}}\left|\triangle_{\ell}^{j} f(y)\right|^{2}\right)^{1 / 2}\right\|_{L^{p}\left(\mathbb{R}^{2}\right)}^{p} \\
& \lesssim\left\|\mathcal{M}_{j, l o c}^{k}\right\|_{L^{p} \rightarrow L^{p}}^{p}\|f\|_{L^{p}\left(\mathbb{R}^{2}\right)}
\end{aligned}
$$

Based on the above argument, next we will only consider

$$
\sum_{k} 2^{-k} \sum_{j \geq 1}\left\|\mathcal{M}_{j, l o c}^{k}\right\|_{L^{p} \rightarrow L^{p}} \leq C_{p} .
$$

In order to get (2.34), first we will estimate

$$
\widehat{d \mu_{k}}\left(\delta_{t} \xi\right)=\int_{\mathbb{R}} e^{-i t^{2} \xi_{2}\left(-s x+x^{2} \phi(\delta x)\right)} \tilde{\rho}(x) \eta(\delta x) d x,
$$

where $2^{-k}=\delta$ and

$$
s:=s(\xi, t)=-\frac{\xi_{1}}{t \xi_{2}}, \text { for } \xi_{2} \neq 0
$$


If $\xi_{2}=0$, then

$$
\left|\widehat{d \mu_{k}}\left(\delta_{t} \xi\right)\right|=\left|(\eta(\delta \cdot) \tilde{\rho})^{\wedge}\left(t \xi_{1}\right)\right| \leq \frac{C_{N}^{\prime}}{\left(1+\left|t \xi_{1}\right|\right)^{N}}=\frac{C_{N}^{\prime}}{\left(1+\left|\delta_{t} \xi\right|\right)^{N}}
$$

and for multi-index $\alpha$,

$$
\left|D_{\xi}^{\alpha} \widehat{d \mu_{k}}\left(\delta_{t} \xi\right)\right|=\left|D_{\xi}^{\alpha}(\eta(\delta \cdot) \tilde{\rho})^{\wedge}\left(t \xi_{1}\right)\right| \leq \frac{C_{\alpha, N}}{\left(1+\left|\delta_{t} \xi\right|\right)^{N}} .
$$

Since $t \approx 1$, then we can deduce the case $\xi_{2}=0$ into $B_{k}$ of the following (2.39).

Put

$$
\Phi(s, x, \delta)=-s x+x^{2} \phi(\delta x)
$$

then we have

$$
\partial_{x} \Phi(s, x, \delta)=-s+2 x \phi(\delta x)+x^{2} \delta \phi^{\prime}(\delta x)
$$

and

$$
\partial_{x}^{2} \Phi(s, x, \delta)=2 \phi(\delta x)+4 x \delta \phi^{\prime}(\delta x)+x^{2} \delta^{2} \phi^{\prime \prime}(\delta x) .
$$

Since $k$ is sufficiently large and $\phi(0) \neq 0$, then the implicit function theorem implies that there exists a smooth solution $x_{c}=\tilde{q}(s, \delta)$ of the equation $\partial_{x} \Phi(s, x, \delta)=0$. For the sake of simplicity, we may assume $\phi(0)=1 / 2$.

Meanwhile, we observe when $k$ tends to infinity, $\tilde{q}(s, \delta)$ smoothly converges to the solution $\tilde{q}(s, 0)=s$ of the equation $\partial_{x} \Phi(s, x, 0)=0$.

Let $\tilde{\Phi}(s, \delta):=\Phi(s, \tilde{q}(s, \delta), \delta)$. From the above arguments and Taylor expansion of smooth functions $\tilde{q}(s, \delta)$ and $\phi(x)$, the phase function

$$
-t^{2} \xi_{2} \tilde{\Phi}(s, \delta)=\frac{\xi_{1}^{2}}{2 \xi_{2}}+\delta \frac{\xi_{1}^{3}}{t \xi_{2}^{2}} \phi^{\prime}(0)+\delta^{2} R(t, \xi, \delta),
$$

where $R(t, \xi, \delta)$ is homogeneous of degree one in $\xi \cdot-t^{2} \xi_{2} \tilde{\Phi}(s, \delta)$ can be considered as a small perturbation of $\frac{\xi_{1}^{2}}{2 \xi_{2}}+\delta \frac{\xi_{1}^{3}}{t \xi_{2}^{2}} \phi^{\prime}(0)$.

By applying the method of stationary phase, we have

$$
\widehat{d \mu_{k}}\left(\delta_{t} \xi\right)=e^{-i t^{2} \xi_{2} \tilde{\Phi}(s, \delta)} \chi_{k}\left(\frac{\xi_{1}}{t \xi_{2}}\right) \frac{A_{k}\left(\delta_{t} \xi\right)}{\left(1+\left|\delta_{t} \xi\right|\right)^{1 / 2}}+B_{k}\left(\delta_{t} \xi\right)
$$

where $\chi_{k}$ is a smooth function supported in the interval $\left[c_{k}, \tilde{c}_{k}\right]$, for certain non-zero positive constants $c_{1} \leq c_{k}, \tilde{c_{k}} \leq c_{2}$ depending only on $k .\left\{A_{k}\left(\delta_{t} \xi\right)\right\}_{k}$ is contained in a bounded subset of symbols of order zero. More precisely, for arbitrary $t \in[1,2]$,

$$
\left|D_{\xi}^{\alpha} A_{k}\left(\delta_{t} \xi\right)\right| \leq C_{\alpha}(1+|\xi|)^{-\alpha}
$$



of curves in the plane

where $C_{\alpha}$ do not depend on $k$ and $t$. Furthermore, $B_{k}$ is a remainder term and satisfies for arbitrary $t \in[1,2]$,

$$
\left|D_{\xi}^{\alpha} B_{k}\left(\delta_{t} \xi\right)\right| \leq C_{\alpha, N}(1+|\xi|)^{-N}
$$

where $C_{\alpha, N}$ are admissible constants and again do not depend on $k$ and $t$.

First, let us consider the remainder part of (2.34). Set

$$
M_{j}^{k, 0} f(y):=\sup _{t \in[1,2]}\left|\int_{\mathbb{R}^{2}} e^{i \xi \cdot y} B_{k}\left(\delta_{t} \xi\right) \beta\left(2^{-j}\left|\delta_{t} \xi\right|\right) \hat{f}(\xi) d \xi\right|
$$

By (2.41) and integration by parts, it is easy to get $\left|\left(B_{k} \beta\left(2^{-j} \cdot\right)\right)^{\vee}(x)\right| \leq C_{N} 2^{-j N}(1+$ $|x|)^{-N}$. So we have that

$$
\begin{aligned}
\left\|M_{j}^{k, 0}\right\|_{L^{p} \rightarrow L^{p}} & =\sup \left\{\left\|M_{j}^{k, 0} f\right\|_{L^{p}}:\|f\|_{L^{p}}=1\right\} \\
& =\sup \left\{\left\|\sup _{t \in[1,2]}\left|t^{-3}\left(B_{k} \beta\left(2^{-j} \cdot\right)\right)^{\vee}\left(\delta_{t^{-1}} \cdot\right) * f\right|\right\|_{L^{p}}:\|f\|_{L^{p}}=1\right\} \\
& \leq \sup \left\{C_{N} 2^{-j N}\|M f\|_{L^{p}}:\|f\|_{L^{p}}=1\right\} \\
& \leq C_{N} 2^{-j N},
\end{aligned}
$$

where $M$ is the Hardy-Littlewood maximal operator defined by $(2.32)$, and the $L^{p}$ boundedness $(1<p<\infty)$ of $M$ implies (2.24) for remainder part of (2.34).

Put

$$
A_{t, j}^{k} f(y):=\int_{\mathbb{R}^{2}} e^{i\left(\xi \cdot y-t^{2} \xi_{2} \tilde{\Phi}(s, \delta)\right)} \chi_{k}\left(\frac{\xi_{1}}{t \xi_{2}}\right) \frac{A_{k}\left(\delta_{t} \xi\right)}{\left(1+\left|\delta_{t} \xi\right|\right)^{1 / 2}} \beta\left(2^{-j}\left|\delta_{t} \xi\right|\right) \hat{f}(\xi) d \xi
$$

Denote by $M_{j}^{k, 1}$ the corresponding maximal operator over [1,2]. It remains to prove that

$$
\sum_{k} 2^{-k} \sum_{j \geq 1}\left\|M_{j}^{k, 1}\right\|_{L^{p} \rightarrow L^{p}} \leq C_{p, N}
$$

Since $\tilde{\Phi}(s, \delta)$ is homogeneous of degree zero in $\xi$ and $\frac{\xi_{1}}{\xi_{2}} \approx 1$, then

$$
\left|\nabla_{\xi}\left[\xi \cdot(y-x)-t^{2} \xi_{2} \tilde{\Phi}(s, \delta)\right]\right| \geq C|y-x|
$$

provided $|y-x| \geq L$, where $L$ is very large and determined by $c_{1}, c_{2}$ and $\|\phi\|_{\infty(I)}$. By integration by parts, we will see that the kernel of the operator $A_{t, j}^{k}$ is dominated by $2^{-j N} \mathcal{O}\left(|y-x|^{-N}\right)$ if $|y-x| \geq L$. From now on, we will restrict our view on the situation

$$
|y-x| \leq L .
$$


Let $B_{i}(L)$ be a ball with center $i$ and radius $L$. Furthermore, we will show that

$$
\sup \left\{\left\|M_{j}^{k, 1} f\right\|_{L^{p}}:\|f\|_{L^{p}}=1, \operatorname{supp} f \subset B_{0}(L)\right\} \leq C_{p} 2^{-j \tilde{\epsilon}_{1}(p)} 2^{k \tilde{\epsilon}_{2}(p)}
$$

implies that

$$
\left\|M_{j}^{k, 1}\right\|_{L^{p} \rightarrow L^{p}} \leq C_{p} 2^{-j \tilde{\epsilon}_{1}(p)} 2^{k \tilde{\epsilon}_{2}(p)},
$$

where $C_{p}$ depends on $p, c_{1}, c_{2}$ and $\|\phi\|_{\infty(I)}$, and $\tilde{\epsilon}_{1}(p), \tilde{\epsilon}_{2}(p)>0$. Then in order to prove inequality (2.34), it suffices to prove inequality (2.46).

In fact, we can decompose $f=\sum_{i \in L \mathbb{Z}^{2}} f_{i}$, where supp $f_{i} \subset B_{i}(L)$. If we have proved (2.46), then

$$
\begin{aligned}
\left\|M_{j}^{k, 1} f\right\|_{L^{p}} & \leq\left\|\sum_{i \in L \mathbb{Z}^{2}} M_{j}^{k, 1} f_{i}\right\|_{L^{p}} \\
& \leq\left(\sum_{i \in L \mathbb{Z}^{2}}\left\|M_{j}^{k, 1}\left(f_{i}(\cdot+i)\right)\right\|_{L^{p}}^{p}\right)^{1 / p} \\
& \leq C_{p} 2^{-j \tilde{\epsilon}_{1}(p)} 2^{k \tilde{\epsilon}_{2}(p)}\left(\sum_{i \in L \mathbb{Z}^{2}}\left\|f_{i}(\cdot+i)\right\|_{L^{p}}^{p}\right)^{1 / p} \\
& \leq C_{p} 2^{-j \tilde{\epsilon}_{1}(p)} 2^{k \tilde{\epsilon}_{2}(p)}\|f\|_{L^{p}}
\end{aligned}
$$

which implies (2.47).

Now we observe inequality (2.46), together with the assumption (2.45), we can choose $\rho_{1} \in C_{0}^{\infty}\left(\mathbb{R}^{2} \times\left[\frac{1}{2}, 4\right]\right)$ such that $(2.46)$ will follow from that

$$
\left\|\widetilde{M_{j}^{k, 1}}\right\|_{L^{p} \rightarrow L^{p}} \leq C_{p} 2^{-j \tilde{\epsilon}_{1}(p)} 2^{k \tilde{\epsilon}_{2}(p)},
$$

where

$$
\widetilde{M_{j}^{k, 1}} f(y):=\sup _{t \in[1,2]}\left|\rho_{1}(y, t) A_{t, j}^{k} f(y)\right| .
$$

Lemma 2.3.1.

$$
\left\|\widetilde{M_{j}^{k, 1}} f\right\|_{L^{p}} \leq C_{p} 2^{-(j \wedge k) / p}\|f\|_{L^{p},} \quad 2 \leq p \leq \infty
$$

Proof. To prove the above lemma, we employ the M. Riesz interpolation theorem between the $L^{2}$ and the $L^{\infty}$-estimate. By Lemma 2.2.3, we have

$$
\begin{aligned}
& \left\|\widetilde{M_{j}^{k, 1}} f\right\|_{L^{2}}^{2} \\
& \leq\left(\int_{1 / 2}^{4} \int_{\mathbb{R}^{2}}\left|\rho_{1}(y, t) A_{t, j}^{k} f(y)\right|^{2} d y d t\right)^{1 / 2}\left(\int_{1 / 2}^{4} \int_{\mathbb{R}^{2}}\left|\frac{\partial}{\partial t}\left(\rho_{1}(y, t) A_{t, j}^{k} f(y)\right)\right|^{2} d y d t\right)^{1 / 2} \\
& =\|\| \rho_{1}(y, t) A_{t, j}^{k} f(y)\left\|_{\left(L^{2}, d y\right)}\right\|_{\left(L^{2}, d t\right)}\|\| \frac{\partial}{\partial t}\left(\rho_{1}(y, t) A_{t, j}^{k} f(y)\right)\left\|_{\left(L^{2}, d y\right)}\right\|_{\left(L^{2}, d t\right)} .
\end{aligned}
$$


Moreover,

$$
\frac{\partial}{\partial t}\left(\rho_{1}(y, t) A_{t, j}^{k} f(y)\right)=\int_{\mathbb{R}^{2}} e^{i\left(\xi \cdot y-t^{2} \xi_{2} \tilde{\Phi}(s, \delta)\right)} h_{k}(y, t, \xi, j) \hat{f}(\xi) d \xi
$$

where

$$
\begin{aligned}
h_{k}(y, t, \xi, j)= & \left(\frac{\partial}{\partial t} \rho_{1}(y, t)+i \frac{\partial}{\partial t}\left(-t^{2} \xi_{2} \tilde{\Phi}(s, \delta)\right) \rho_{1}(y, t)\right) \chi_{k}\left(\frac{\xi_{1}}{t \xi_{2}}\right) \frac{A_{k}\left(\delta_{t} \xi\right)}{\left(1+\left|\delta_{t} \xi\right|\right)^{1 / 2}} \beta\left(2^{-j}\left|\delta_{t} \xi\right|\right) \\
& +\rho_{1}(y, t) \frac{\partial}{\partial t}\left(\chi_{k}\left(\frac{\xi_{1}}{t \xi_{2}}\right)\right) \frac{A_{k}\left(\delta_{t} \xi\right)}{\left(1+\left|\delta_{t} \xi\right|\right)^{1 / 2}} \beta\left(2^{-j}\left|\delta_{t} \xi\right|\right) \\
& +\rho_{1}(y, t) \chi_{k}\left(\frac{\xi_{1}}{t \xi_{2}}\right) \frac{\partial}{\partial t}\left(\frac{A_{k}\left(\delta_{t} \xi\right)}{\left(1+\left|\delta_{t} \xi\right|\right)^{1 / 2}}\right) \beta\left(2^{-j}\left|\delta_{t} \xi\right|\right) \\
& +\rho_{1}(y, t) \chi_{k}\left(\frac{\xi_{1}}{t \xi_{2}}\right) \frac{A_{k}\left(\delta_{t} \xi\right)}{\left(1+\left|\delta_{t} \xi\right|\right)^{1 / 2}} \frac{\partial}{\partial t}\left(\beta\left(2^{-j}\left|\delta_{t} \xi\right|\right)\right) .
\end{aligned}
$$

From (2.38) and (2.40), we get $\left|\frac{\partial}{\partial t}\left(-t^{2} \xi_{2} \tilde{\Phi}(s, \delta)\right)\right|=\left|-\delta \frac{\xi_{1}^{3}}{t^{2} \xi_{2}^{2}} \phi^{\prime}(0)+\delta^{2} \frac{\partial}{\partial t} R(t, \xi, \delta)\right| \lesssim$ $\delta 2^{j},\left|\frac{\partial}{\partial t}\left(\chi_{k}\left(\frac{\xi_{1}}{t \xi_{2}}\right)\right)\right| \approx 1,\left|\frac{\partial}{\partial t}\left(\frac{A_{k}\left(\delta_{t} \xi\right)}{\left(1+\left|\delta_{t} \xi\right|\right)^{1 / 2}}\right)\right| \lesssim 2^{-j / 2}$ and $\left|\frac{\partial}{\partial t}\left(\beta\left(2^{-j}\left|\delta_{t} \xi\right|\right)\right)\right| \lesssim 1$. Therefore $\left|h_{k}(y, t, \xi, j)\right| \lesssim 2^{j / 2} \delta+2^{-j / 2}$. Then $\left(2^{j / 2} \delta+2^{-j / 2}\right)^{-1} \frac{\partial}{\partial t}\left(\rho_{1}(y, t) A_{t, j}^{k} f(y)\right)$ behaves like $2^{j / 2} A_{t, j}^{k}$ which is a symbol of order zero. So we only consider the $L^{2}$-boundedness of the operator $2^{j / 2} \rho A_{t, j}^{k}$, for fixed $t \in[1 / 2,4]$, however this is easily obtained by the Plancherel theorem, i.e.

$$
\begin{aligned}
\left\|2^{j / 2} \rho_{1}(y, t) A_{t, j}^{k} f(y)\right\|_{\left(L^{2}, d y\right)} & \leq\left\|e^{-i t^{2} \xi_{2} \tilde{\Phi}(s, \delta)} 2^{j / 2} \chi_{k}\left(\frac{\xi_{1}}{t \xi_{2}}\right) \frac{A_{k}\left(\delta_{t} \xi\right)}{\left(1+\left|\delta_{t} \xi\right|\right)^{1 / 2}} \beta\left(2^{-j}\left|\delta_{t} \xi\right|\right) \hat{f}(\xi)\right\|_{\left(L^{2}, d \xi\right)} \\
& \leq C\|f\|_{L^{2}} .
\end{aligned}
$$

From the above arguments, we obtain

$$
\begin{aligned}
\left\|\widetilde{M_{j}^{k, 1}} f\right\|_{L^{2}}^{2} \leq & 2^{-j / 2}\left(2^{j / 2} \delta+2^{-j / 2}\right)\|\| 2^{j / 2} \rho_{1}(y, t) A_{t, j}^{k} f(y)\left\|_{\left(L^{2}, d y\right)}\right\|_{\left(L^{2}, d t\right)} \\
& \times\|\| \frac{\partial}{\partial t}\left(\left(2^{j / 2} \delta+2^{-j / 2}\right)^{-1} \rho_{1}(y, t) A_{t, j}^{k} f(y)\right)\left\|_{\left(L^{2}, d y\right)}\right\|_{\left(L^{2}, d t\right)} \\
\leq & C 2^{-j / 2}\left(2^{j / 2} \delta+2^{-j / 2}\right)\|f\|_{L^{2}}^{2} \\
\leq & C\left(\delta+2^{-j}\right)\|f\|_{L^{2}}^{2},
\end{aligned}
$$

then

$$
\widetilde{\| M_{j}^{k, 1}} f\left\|_{L^{2}} \leq C\left(\delta+2^{-j}\right)^{1 / 2}\right\| f \|_{L^{2}} .
$$

Next, let us turn to prove

$$
\left\|\widetilde{M_{j}^{k, 1}} f\right\|_{L^{\infty}} \leq C\|f\|_{L^{\infty}}
$$


Inequality (2.52) follows from the fact that the kernels of $A_{t, j}^{k}$ are uniformly bounded in $L^{1}\left(\mathbb{R}^{2}\right)$. The idea of the proof can be found in page 406-408 in [41]. For completeness, in fact, this idea also will be used later, so we give a brief overview here.

First, we introduce the angular decomposition as in [41] of the $\xi$-space in the plane. For each positive integer $j$, we consider a roughly equally spaced set of points with grid length $2^{-j / 2}$ on the unit circle $S^{1}$; that is, we fix a collection $\left\{\xi_{j}^{\nu}\right\}_{\nu}$ of unit vectors, that satisfy:

(a) $\left|\xi_{j}^{\nu}-\xi_{j}^{\nu^{\prime}}\right| \geq 2^{-j / 2}$, if $\nu \neq \nu^{\prime}$;

(b) if $\xi \in S^{1}$, then there exists a $\xi_{j}^{\nu}$ so that $\left|\xi-\xi_{j}^{\nu}\right|<2^{-j / 2}$.

Let $\Gamma_{j}^{\nu}$ denote the corresponding cone in the $\xi$-space whose central direction is $\xi_{j}^{\nu}$, i.e.

$$
\Gamma_{j}^{\nu}=\left\{\xi:|\xi /| \xi\left|-\xi_{j}^{\nu}\right| \leq 2 \cdot 2^{-j / 2}\right\}
$$

We can construct an associated partition of unity:

Lemma 2.3.2. [41] $\chi_{j}^{\nu}$ is homogeneous of degree zero in $\xi$ and supported in $\Gamma_{j}^{\nu}$, with

$$
\sum_{\nu} \chi_{j}^{\nu}(\xi)=1 \quad \text { for all } \xi \neq 0 \text { and all } j
$$

and

$$
\left|\partial_{\xi}^{\alpha} \chi_{j}^{\nu}(\xi)\right| \leq A_{\alpha} 2^{|\alpha| j / 2}|\xi|^{-|\alpha|}
$$

Hence, in order to establish (2.52), it is sufficient to prove

$$
\int_{\mathbb{R}^{2}}\left|K_{t}^{\nu}(y)\right| d y \leq C 2^{-j / 2}
$$

where $C$ does not depend on $t, j, k$ and $\nu$, and

$$
K_{t}^{\nu}(y)=\rho_{1}(y, t) \int_{\mathbb{R}^{2}} e^{i\left(\xi \cdot y-t^{2} \xi_{2} \tilde{\Phi}(s, \delta)\right)} \widetilde{A_{k}}(\xi, t) \beta\left(2^{-j}\left|\delta_{t} \xi\right|\right) \chi_{j}^{\nu}(\xi) d \xi
$$

and $\widetilde{A_{k}}(\xi, t)=\chi_{k}\left(\frac{\xi_{1}}{t \xi_{2}}\right) \frac{A_{k}\left(\delta_{t} \xi\right)}{\left(1+\left|\delta_{t} \xi\right|\right)^{1 / 2}}$.

Then we have

$$
\begin{aligned}
\widetilde{M_{j}^{k, 1} f} \|_{L^{\infty}} & :=\left\|\sup _{t>0}\left|K_{t} * f\right|\right\|_{L^{\infty}} \\
& \leq\|f\|_{L^{\infty}} \sup _{t>0} \sum_{\nu \sum^{j / 2}}\left\|K_{t}^{\nu}\right\|_{L^{1}} \\
& \lesssim\|f\|_{L^{\infty}}
\end{aligned}
$$


and get inequality (2.52).

For fixed $\nu$, the inner integral (2.56) is supported in the truncated cone $\Gamma_{j}^{\nu}$. Let $T$ be the transpose operator, i.e. $T\left(x_{1}, x_{2}\right)=\left(x_{1}, x_{2}\right)^{\mathrm{t}}$. We can find a rotation $\rho_{j}^{\nu}$ such that $T^{-1} \rho_{j}^{\nu} T(1,0)=\xi_{j}^{\nu}$. Write $\tilde{T}=T^{-1} \rho_{j}^{\nu} T$ and $\tilde{T}_{i}$ the $i$-th variable after the action of $\tilde{T}$, this leads to prove the following estimate

$$
\int_{\mathbb{R}^{2}}\left|\rho_{1}(y, t) \int_{\mathbb{R}^{2}} e^{i\left(\tilde{T} \xi \cdot y-t^{2} \tilde{T}_{2} \xi \tilde{\Phi}(\tilde{s}, \delta)\right)} \widetilde{A_{k}}(\tilde{T} \xi, t) \beta\left(2^{-j}\left|\delta_{t} \tilde{T} \xi\right|\right) \chi_{j}^{\nu}(\tilde{T} \xi) d \xi\right| d y \leq C 2^{-j / 2}
$$

where $\tilde{s}=-\frac{\tilde{T}_{1} \xi}{t \tilde{T}_{2} \xi}$. From the $\xi$-support of the inner integral of (2.57), we know that $\xi$ belongs to the following region, see Figure 1.

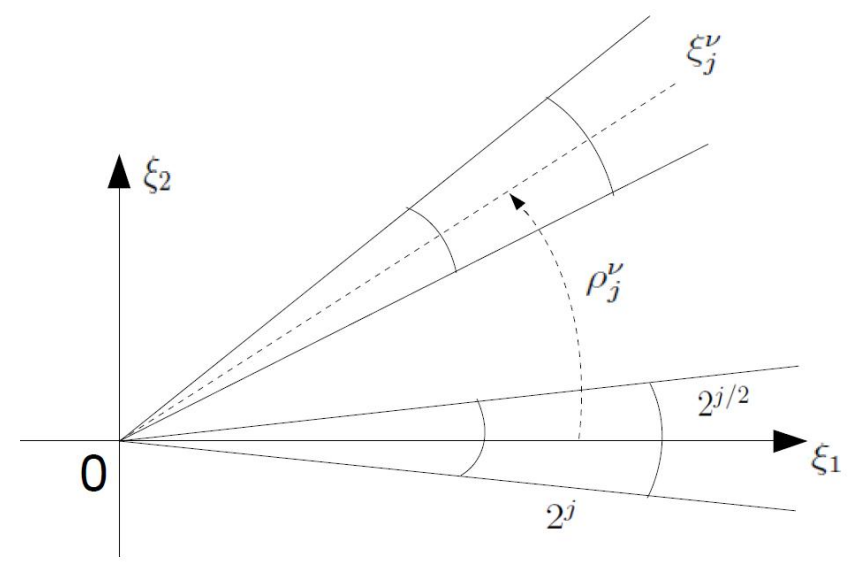

Figure 1

Put $\tilde{\tilde{\Phi}}(y, \xi)=\tilde{T} \xi \cdot y-t^{2} \tilde{T}_{2} \xi \tilde{\Phi}(\tilde{s}, \delta)$. Clealy $\tilde{\tilde{\Phi}}$ is homogeneous of degree one in $\xi$, hence $\tilde{\tilde{\Phi}}(y, \xi)=\xi \cdot \nabla_{\xi} \tilde{\tilde{\Phi}}(y, \xi)$. Let $h(\xi)=\tilde{\tilde{\Phi}}(y, \xi)-\xi \cdot \nabla_{\xi} \tilde{\tilde{\Phi}}(y, \bar{\xi})$, where $\bar{\xi}=(1,0)$. We claim that

$$
\begin{aligned}
& \left|\left(\frac{\partial}{\partial \xi_{1}}\right)^{N} h(\xi)\right| \leq A_{N} \cdot 2^{-j N}, \\
& \left|\left(\frac{\partial}{\partial \xi_{2}}\right)^{N} h(\xi)\right| \leq A_{N} \cdot 2^{-j N / 2} .
\end{aligned}
$$

Since $h(\xi)$ is also homogeneous of degree one, then $h(\bar{\xi})=0$ and $\nabla_{\xi} h(\bar{\xi})=0$. As a result

$$
\left(\frac{\partial}{\partial \xi_{1}}\right)^{N} h(\bar{\xi})=\frac{\partial}{\partial \xi_{2}}\left(\frac{\partial}{\partial \xi_{1}}\right)^{N} h(\bar{\xi})=0
$$


Thus $\left(\frac{\partial}{\partial \xi_{1}}\right)^{N} h(\xi)=\mathcal{O}\left(\frac{\left|\xi_{2}\right|^{2}}{|\xi|^{N+1}}\right)$. Since $\left|\xi_{2}\right| \lesssim 2^{j / 2}$ and $|\xi| \approx 2^{j}$, we have that $\frac{\left|\xi_{2}\right|^{2}}{|\xi|^{N+1}} \leq$ $\mathcal{O}\left(2^{-j N}\right)$, we get inequality $(2.58)$.

Again, $\frac{\partial}{\partial \xi_{2}} h(\bar{\xi})=0$, so $\frac{\partial}{\partial \xi_{2}} h(\xi)=\mathcal{O}\left(\frac{\xi_{2}}{|\xi|}\right) \approx 2^{-j / 2}$. For $N>1$, we have that $\left|\left(\frac{\partial}{\partial \xi_{2}}\right)^{N} h(\xi)\right| \leq$ $A_{N}|\xi|^{1-N}$, by homogeneity, and $2^{j(1-N)} \leq 2^{-N j / 2}$, so we finish the proof of inequality $(2.59)$.

We observe that $\chi_{j}^{\nu} \circ \tilde{T}$ satisfies the same conditions (2.53) and (2.54) as $\chi_{j}^{\nu}$. Meanwhile, $\operatorname{supp}\left(\chi_{j}^{\nu} \circ \tilde{T}\right) \subset\left\{\xi:\left|\frac{\xi}{|\xi|}-(1,0)\right| \leq 2 \cdot 2^{-j / 2}\right\}$. If we set $\epsilon=2^{-j / 2}$ in (iii) of Lemma 14 in [23], then it follows that (2.54) has an improvement for $\chi_{j}^{\nu} \circ \tilde{T}$, i.e.

$$
\left|\left(\frac{\partial}{\partial \xi_{1}}\right)^{N}\left(\chi_{j}^{\nu} \circ \tilde{T}\right)(\xi)\right| \leq C_{N}|\xi|^{-N} \approx 2^{-j N} .
$$

We now rewrite

$$
K_{t}^{\nu}(y)=\rho_{1}(y, t) \int_{\mathbb{R}^{2}} e^{i \xi \cdot \nabla_{\xi} \tilde{\tilde{\Phi}}(y, \bar{\xi})} e^{i h(\xi)} \widetilde{A_{k}}(\tilde{T} \xi, t) \beta\left(2^{-j}\left|\delta_{t} \tilde{T} \xi\right|\right)\left(\chi_{j}^{\nu} \circ \tilde{T}\right)(\xi) d \xi .
$$

Next, we introduce the operator $\mathcal{L}$ defined by $\mathcal{L}=I-2^{2 j} \frac{\partial^{2}}{\partial \xi_{1}^{2}}-2^{j} \frac{\partial^{2}}{\partial \xi_{2}^{2}}$. Because of $(2.54)$, (2.60) and the fact that $\widetilde{A_{k}}$ is a symbol of order $-1 / 2$, we get that

$$
\left|\mathcal{L}^{N}\left(e^{i h(\xi)} \widetilde{A_{k}}(\tilde{T} \xi, t) \beta\left(2^{-j}\left|\delta_{t} \tilde{T} \xi\right|\right)\left(\chi_{j}^{\nu} \circ \tilde{T}\right)(\xi)\right)\right| \leq A_{N} 2^{-j / 2}
$$

However,

$$
\mathcal{L}^{N} e^{i \xi \cdot \nabla_{\xi} \tilde{\tilde{\Phi}}(y, \bar{\xi})}=\left(1+2^{2 j}\left|\frac{\partial}{\partial \xi_{1}} \tilde{\tilde{\Phi}}(y, \bar{\xi})\right|^{2}+2^{j}\left|\frac{\partial}{\partial \xi_{2}} \tilde{\tilde{\Phi}}(y, \bar{\xi})\right|^{2}\right)^{N} \cdot e^{i \xi \cdot \nabla_{\xi} \tilde{\tilde{\Phi}}(y, \bar{\xi})}
$$

From Figure 1, we note that the support of the integrand has volume at most $\mathcal{O}\left(2^{j}\right.$. $\left.2^{j / 2}\right) \approx 2^{3 j / 2}$, thus we obtain by integration by parts that

$$
\left|K_{t}^{\nu}(y)\right| \leq A_{N} 2^{3 j / 2} 2^{-j / 2}\left(1+2^{2 j}\left|\frac{\partial}{\partial \xi_{1}} \tilde{\tilde{\Phi}}(y, \bar{\xi})\right|^{2}+2^{j}\left|\frac{\partial}{\partial \xi_{2}} \tilde{\tilde{\Phi}}(y, \bar{\xi})\right|^{2}\right)^{-N} .
$$

In order to prove $(2.55)$, we make the change of variables $y \mapsto\left(\frac{\partial}{\partial \xi_{1}} \tilde{\tilde{\Phi}}(y, \bar{\xi}), \frac{\partial}{\partial \xi_{2}} \tilde{\tilde{\Phi}}(y, \bar{\xi})\right)$. Since

$$
\operatorname{det}\left(\frac{\partial^{2} \tilde{\tilde{\Phi}}(y, \bar{\xi})}{\partial \xi_{i} \partial y_{i}}\right) \neq 0,
$$

and the Jacobian is bounded from below, then we have

$$
\int_{\mathbb{R}^{2}}\left|K_{t}^{\nu}(y)\right| d y \leq A_{N} 2^{j} \int_{\mathbb{R}^{2}} \frac{d y}{\left(1+\left|2^{j} y_{1}\right|+\left|2^{j / 2} y_{2}\right|\right)^{2 N}} \leq C 2^{-j / 2} .
$$



of curves in the plane

Now we split the set of $j$ into two parts $j>2 k$ and $j \leq 2 k$. By Lemma 2.3.1, we get

$$
\sum_{k} 2^{-k} \sum_{j \leq 2 k}\left\|\widetilde{M_{j}^{k, 1}}\right\|_{L^{p} \rightarrow L^{p}} \leq C_{p}
$$

For $j>2 k$, we introduce some new notations. Let $j=k+j^{\prime}$, then $j^{\prime}>k$. Set $\lambda=2^{j^{\prime}}>2^{k}=\delta^{-1}$, then $2^{j}=\frac{\lambda}{\delta}$. By Lemma 2.2.3,

$$
\begin{aligned}
\| \widetilde{M_{j}^{k, 1}} & f \|_{L^{4}}^{4} \\
\leq & C \frac{\delta^{2}}{\lambda}\left(\int_{\mathbb{R}^{2}} \int_{1 / 2}^{4}\left|\rho_{1}(y, t) \int_{\mathbb{R}^{2}} e^{i\left(\xi \cdot y-t^{2} \xi_{2} \tilde{\Phi}(s, \delta)\right)}(\lambda / \delta)^{1 / 2} \widetilde{A_{k}}(\xi, t) \beta\left(\frac{\delta}{\lambda}\left|\delta_{t} \xi\right|\right) \hat{f}(\xi) d \xi\right| d y d t\right)^{3 / 4} \\
& \times\left(\int_{\mathbb{R}^{2}} \int_{1 / 2}^{4}\left|\frac{\partial}{\partial t}\left(\rho_{1}(y, t) \int_{\mathbb{R}^{2}} e^{i\left(\xi \cdot y-t^{2} \xi_{2} \tilde{\Phi}(s, \delta)\right)}(\delta \lambda)^{-1 / 2} \widetilde{A_{k}}(\xi, t) \beta\left(\frac{\delta}{\lambda}\left|\delta_{t} \xi\right|\right) \hat{f}(\xi) d \xi\right)\right|^{4} d y d t\right)^{1 / 4} .
\end{aligned}
$$

In order to simplify the notations, we choose $\tilde{\chi} \in C_{0}^{\infty}\left(\left[c_{1}, c_{2}\right]\right)$ so that $\tilde{\chi}\left(\frac{\xi_{1}}{\xi_{2}}\right) \chi\left(\frac{\xi_{1}}{t \xi_{2}}\right)=$ $\chi\left(\frac{\xi_{1}}{t \xi_{2}}\right)$ for arbitrary $t \in[1 / 2,4]$ and $k$ sufficiently large. In a similar way we choose $\rho_{0} \in C_{0}^{\infty}((-10,10))$ such that $\rho_{0}(|\xi|) \beta\left(\left|\delta_{t} \xi\right|\right)=\beta\left(\left|\delta_{t} \xi\right|\right)$ for arbitrary $t \in[1 / 2,4]$. Furthermore, since $A_{k}$ satisfies $(2.40)$, if $a(\xi, t):=(\lambda / \delta)^{1 / 2} \widetilde{A}_{k}(\xi, t) \beta\left(\frac{\delta}{\lambda}\left|\delta_{t} \xi\right|\right)$ for $k$ sufficiently large, then $a(\xi, t)$ is a symbol of order zero, i.e. for any $t \in[1 / 2,4], \alpha \in \mathbb{N}^{2}$,

$$
\left|\left(\frac{\partial}{\partial \xi}\right)^{\alpha} a(\xi, t)\right| \leq C_{\alpha}(1+|\xi|)^{-|\alpha|}
$$

Assume that we have obtained the following theorem:

Theorem 2.3.3. For all $\lambda>1 / \delta$,

$$
\left(\int_{\mathbb{R}^{2}} \int_{1 / 2}^{4}\left|\tilde{F}_{\lambda}^{\delta} f(y, t)\right|^{4} d y d t\right)^{1 / 4} \leq C \lambda^{1 / 8+\epsilon_{1}} \delta^{-\left(1 / 2+\epsilon_{2}\right)}\|f\|_{L^{4}\left(\mathbb{R}^{2}\right)} \text {, some } \epsilon_{1}, \epsilon_{2}>0
$$

where

$$
\tilde{F}_{\lambda}^{\delta} f(y, t)=\rho_{1}(y, t) \int_{\mathbb{R}^{2}} e^{i\left(\xi \cdot y-t^{2} \xi_{2} \tilde{\Phi}(s, \delta)\right)} a(\xi, t) \rho_{0}\left(\frac{\delta}{\lambda}|\xi|\right) \tilde{\chi}\left(\frac{\xi_{1}}{\xi_{2}}\right) \hat{f}(\xi) d \xi
$$

Remark 2.3.1. If we replace the localized frequency $|\xi| \approx \lambda$ of Theorem 3.3 in [18] by $|\xi| \approx \frac{\lambda}{\delta}$, then we obtain the factor $\lambda^{1 / 8+\epsilon} 2^{5 k / 8+\epsilon}$ which is larger than the factor $\lambda^{1 / 8+\epsilon_{1}} 2^{k / 2+\epsilon_{2}}$ appeared in the above Theorem 2.3.3. This means that we get a better $L^{4}$-estimate for Fourier integral operators not satisfying the cinematic curvature uniformly. 
By (2.62), we obtain

$$
\begin{aligned}
\left\|\widetilde{M_{j}^{k, 1}} f\right\|_{L^{4}\left(\mathbb{R}^{2}\right)} & \leq C\left(\frac{\delta^{2}}{\lambda}\right)^{1 / 4} \lambda^{1 / 8+\epsilon_{1}} \delta^{-\left(1 / 2+\epsilon_{2}\right)}\|f\|_{L^{4}\left(\mathbb{R}^{2}\right)} \\
& =C 2^{j^{\prime}\left(-1 / 8+\epsilon_{1}\right)} 2^{k \epsilon_{2}}\|f\|_{L^{4}\left(\mathbb{R}^{2}\right)} \\
& =C 2^{j\left(-1 / 8+\epsilon_{1}\right)} 2^{k\left(1 / 8-\epsilon_{1}+\epsilon_{2}\right)}\|f\|_{L^{4}\left(\mathbb{R}^{2}\right)} .
\end{aligned}
$$

Employing the M. Riesz interpolation theorem and Lemma 2.3.1, we have for $2<p \leq 4$,

$$
\left\|\widetilde{M_{j}^{k, 1}} f\right\|_{L^{p}\left(\mathbb{R}^{2}\right)} \leq C 2^{j\left(-1 / 8+\epsilon_{1}\right)(2-4 / p)} 2^{k\left(3 / 4-5 /(2 p)+(2-4 / p)\left(\epsilon_{2}-\epsilon_{1}\right)\right)}\|f\|_{L^{p}\left(\mathbb{R}^{2}\right)},
$$

and

$\sum_{k} 2^{-k} \sum_{j>2 k}\left\|\widetilde{M_{j}^{k, 1}}\right\|_{L^{p} \rightarrow L^{p}} \leq C \sum_{k} 2^{-k\left(1 / 4+5 /(2 p)-(2-4 / p)\left(\epsilon_{2}-\epsilon_{1}\right)\right)} \sum_{j>2 k} 2^{j\left(-1 / 8+\epsilon_{1}\right)(2-4 / p)} \leq C_{p}$.

For $4 \leq p<\infty$, the M. Riesz interpolation theorem and Lemma 2.3.1 imply that

$$
\left\|\widetilde{M_{j}^{k, 1}} f\right\|_{L^{p}\left(\mathbb{R}^{2}\right)} \leq C 2^{j\left(-1 / 2+4 \epsilon_{1}\right) / p} 2^{4 k\left(1 / 8-\epsilon_{1}+\epsilon_{2}\right) / p}\|f\|_{L^{p}\left(\mathbb{R}^{2}\right)}
$$

and

$$
\sum_{k} 2^{-k} \sum_{j>2 k}\left\|\widetilde{M_{j}^{k, 1}}\right\|_{L^{p} \rightarrow L^{p}} \leq C \sum_{k} 2^{k\left(1 /(2 p)-1+4\left(\epsilon_{2}-\epsilon_{1}\right) / p\right)} \sum_{j>2 k} 2^{j\left(-1 /(2 p)+4 \epsilon_{1} / p\right)} \leq C_{p} .
$$

We have finished the proof of Theorem 2.1.1.

It remains to prove Theorem 2.3.3. First we introduce a model operator which is translation-invariant. We define it by

$$
\mathcal{F}_{\lambda}^{\delta} f(y, t)=\rho_{1}(y, t) \int_{\mathbb{R}^{2}} e^{i\left(\xi \cdot y+E(\xi)+t q_{\delta}(\xi)\right)} a(\xi, t) \rho_{0}\left(\frac{\delta}{\lambda}|\xi|\right) \tilde{\chi}\left(\frac{\xi_{1}}{\xi_{2}}\right) \hat{f}(\xi) d \xi
$$

where

$$
E(\xi)=\frac{\xi_{1}^{2}}{2 \xi_{2}} \text { and } q_{\delta}(\xi)=\delta \cdot \frac{\xi_{1}^{3}}{\xi_{2}^{2}} \phi^{\prime}(0)
$$

Notice that compared to (2.38), we have replaced $t$ by $1 / t$. This is no problem since $t \approx 1$. Then the model operator $\mathcal{F}_{\lambda}^{\delta}$ approximates the operator $\tilde{\mathcal{F}}_{\lambda}^{\delta}$ whose phase function has of the form (2.38).

Theorem 2.3.4. For all $\lambda>1 / \delta$, we have

$$
\left\|\mathcal{F}_{\lambda}^{\delta} f(y, t)\right\|_{L^{4}\left(\mathbb{R}^{3}\right)} \leq C \lambda^{1 / 8+\epsilon_{1}} \delta^{-\left(1 / 2+\epsilon_{2}\right)}\|f\|_{L^{4}\left(\mathbb{R}^{2}\right)}
$$

We give the proof of Theorem 2.3.4 in Chapter 3. In Chapter 4, the proof of Theorem 2.3.3 will be shown for the phase function $-t^{2} \xi_{2} \tilde{\Phi}(s, \delta)$ in $(2.38)$. 


\subsubsection{The case when $m>1$}

Here we only show the differences with the case $m=1$ and continue to follow the notation used in the previous section. We will mainly estimate

$$
\widehat{d \mu_{k, m}}\left(\delta_{t} \xi\right)=\int_{\mathbb{R}} e^{-i\left(t \xi_{1} x+t^{2} \xi_{2} x^{2} \phi(\delta x)\right)} \tilde{\rho}(x) \eta(\delta x) d x,
$$

where $\delta=2^{-k}$.

Set

$$
s:=s(\xi, t)=-\frac{\xi_{1}}{t \xi_{2}}, \text { for } \xi_{2} \neq 0
$$

and

$$
\Phi(s, x, \delta)=-s x+x^{2} \phi\left(\frac{x}{2^{k}}\right) .
$$

The implicit function theorem implies that for enough large $k$, there exists a smooth solution $\tilde{q}(s, \delta)$ for the equation $\partial_{2} \Phi(s, x, \delta)=0$. Meanwhile, if we choose $k$ sufficiently large and assume $\phi(0)=1 / 2$, then $\tilde{q}(s, \delta)$ smoothly converges to the solution $\tilde{q}(s, 0)=s$ of the equation $\partial_{2} \Phi(s, x, 0)=0$.

Let $\tilde{\Phi}(s, \delta):=\Phi(s, q(s, \delta), \delta)$. The phase function can be written as

$$
-t^{2} \xi_{2} \tilde{\Phi}(s, \delta)=\frac{\xi_{1}^{2}}{2 \xi_{2}}+(-1)^{m+1} \frac{\phi^{(m)}(0)}{m !} \delta^{m} \frac{\xi_{1}^{m+2}}{t^{m} \xi_{2}^{m+1}}+R(t, \xi, \delta)
$$

where $R(t, \xi, \delta)$ is homogeneous of degree one in $\xi$ and has at least $\mathrm{m}+1$ power of $\delta$. Using the similar argument as in the last section, it suffices to prove that

$$
\sum_{k} 2^{-k} \sum_{j \geq 1}\left\|\sup _{t \in[1,2]}\left|\widetilde{A_{t, j}^{k}}\right|\right\|_{L^{p} \rightarrow L^{p}} \leq C_{p}
$$

where

$$
\widetilde{A_{t, j}^{k}} f(y):=\int_{\mathbb{R}^{2}} e^{i \xi \cdot y} \widehat{d \mu_{k, m}}\left(\delta_{t} \xi\right) \beta\left(2^{-j}\left|\delta_{t} \xi\right|\right) \hat{f}(\xi) d \xi
$$

A standard application of the method of stationary phase yields that

$$
\widehat{d \mu_{k, m}}\left(\delta_{t} \xi\right)=e^{-i t^{2} \xi_{2} \tilde{\Phi}(s, \delta)} \chi_{k, m}\left(\frac{\xi_{1}}{t \xi_{2}}\right) \frac{A_{k, m}\left(\delta_{t} \xi\right)}{\left(1+\left|\delta_{t} \xi\right|\right)^{1 / 2}}+B_{k, m}\left(\delta_{t} \xi\right),
$$

where $\chi_{k, m}$ is a smooth function supported in $\left[c_{k, m}, \widetilde{c_{k, m}}\right]$, for certain non-zero constant $c_{k, m}$ and $\widetilde{c_{k, m}}$ dependent only on $k$ and $m$. $A_{k, m}$ is a symbol of order zero in $\xi$ and $\left\{A_{k, m}\left(\delta_{t} \xi\right)\right\}_{k, m}$ is contained in a bounded subset of symbol of order zero. More precisely, for arbitrary $t \in[1,2]$,

$$
\left|D_{\xi}^{\alpha} A_{k, m}\left(\delta_{t} \xi\right)\right| \leq C_{\alpha}(1+|\xi|)^{-\alpha}
$$


where $C_{\alpha}$ do not depend on $k$ and $m$. Furthermore, $B_{k, m}$ is a remaind term and satisfies for arbitrary $t \in[1,2]$,

$$
\left|D_{\xi}^{\alpha} B_{k, m}\left(\delta_{t} \xi\right)\right| \leq C_{\alpha, N}(1+|\xi|)^{-N}
$$

where $C_{\alpha, N}$ are admissible constants and again do not depend on $k$ and $m$.

Put

$$
A_{t, j}^{k} f(y):=\int_{\mathbb{R}^{2}} e^{i\left(\xi \cdot y-t^{2} \xi_{2} \tilde{\Phi}(s, \delta)\right)} \chi_{k, m}\left(\frac{\xi_{1}}{t \xi_{2}}\right) \frac{A_{k, m}\left(\delta_{t} \xi\right)}{\left(1+\left|\delta_{t} \xi\right|\right)^{1 / 2}} \beta\left(2^{-j}\left|\delta_{t} \xi\right|\right) \hat{f}(\xi) d \xi .
$$

A similar discussion as before allows us to choose $\rho_{1}(y, t) \in C_{0}^{\infty}\left(\mathbb{R}^{2} \times[1 / 2,4]\right)$ and to show that it is sufficient to prove that

$$
\sum_{k} 2^{-k} \sum_{j \geq 1}\left\|\widetilde{M_{j}^{k, 1}}\right\|_{L^{p} \rightarrow L^{p}} \leq C_{p}
$$

where

$$
\widetilde{M_{j}^{k, 1}} f(y):=\sup _{t \in[1,2]}\left|\rho_{1}(y, t) A_{t, j}^{k} f(y)\right|,
$$

( compare (2.46), (2.47)).

Moreover,

$$
\frac{\partial}{\partial t}\left(\rho_{1}(y, t) A_{t, j}^{k} f(y)\right)=\int_{\mathbb{R}^{2}} e^{i\left(\xi \cdot y-t^{2} \xi_{2} \tilde{\Phi}(s, \delta)\right)} h_{k, m}(y, t, \xi, j) \hat{f}(\xi) d \xi,
$$

where $\left|h_{k, m}(y, t, \xi, j)\right| \lesssim 2^{j / 2} \delta^{m}+2^{-j / 2}$, which follows from the similar argument with $h_{k}$ in $(2.50)$, together with the facts that $\left|\frac{\partial}{\partial t}\left(-t^{2} \xi_{2} \tilde{\Phi}(s, \delta)\right)\right|=\mid(-1)^{m} \frac{\phi^{(m)}(0)}{m !} \delta^{m} \frac{\xi_{1}^{m+2}}{t^{m+1} \xi_{2}^{m+1}}+$ $\frac{\partial}{\partial t} R(t, \xi, \delta) \mid \lesssim \delta^{m} 2^{j}$.

So we still have the following regularity estimates.

\section{Lemma 2.3.5.}

$$
\left\|\widetilde{M_{j}^{k, 1}} f\right\|_{L^{p}\left(\mathbb{R}^{3}\right)} \leq C_{p} 2^{-(j \wedge k) / p}\|f\|_{L^{p}\left(\mathbb{R}^{2}\right)}, \quad 2 \leq p \leq \infty .
$$

The proof is similar to the one of Lemma 2.3.1. Hence, we have that

$$
\begin{aligned}
\sum_{k} 2^{-k} \sum_{0<j \leq 9 k m}\left\|\widetilde{M_{j}^{k, 1}}\right\|_{L^{p} \rightarrow L^{p}} & \leq C_{p}+\sum_{k} 2^{-k} \sum_{k m<j \leq 9 k m} 2^{-k m / p} \\
& \leq C_{p}+8 m \sum_{k} k 2^{-k(1+m / p)} \lesssim_{p} 1,
\end{aligned}
$$

where $2<p<\infty$.

Based on these results, let us now assume that $j>9 \mathrm{~km}$. We introduce some notations. Let $j=k m+j^{\prime}$ and $\delta^{\prime}=\delta^{m}$, then $\lambda=2^{j^{\prime}}>\delta^{\prime-8}$ and $2^{j}=\frac{\lambda}{\delta^{\prime}}$. After the same simplification as in the previous section, we will prove the following theorem: 

of curves in the plane

Theorem 2.3.6. For $\lambda>\delta^{-8}$, the following inequality

$$
\left(\int_{\mathbb{R}^{2}} \int_{1 / 2}^{4}\left|\tilde{F}_{\lambda}^{\delta^{\prime}} f(y, t)\right|^{4} d y d t\right)^{1 / 4} \leq C \lambda^{1 / 8+\epsilon_{1}} \delta^{\prime-\left(1 / 2+\epsilon_{2}\right)}\|f\|_{L^{4}\left(\mathbb{R}^{2}\right)}
$$

holds true for some $\epsilon_{1}, \epsilon_{2}>0$, where

$$
\tilde{F}_{\lambda}^{\delta^{\prime}} f(y, t)=\rho_{1}(y, t) \int_{\mathbb{R}^{2}} e^{i\left(\xi \cdot y-t^{2} \xi_{2} \tilde{\Phi}(s, \delta)\right)} a(\xi, t) \rho_{0}\left(\frac{\delta^{\prime}}{\lambda}|\xi|\right) \tilde{\chi}\left(\frac{\xi_{1}}{\xi_{2}}\right) \hat{f}(\xi) d \xi
$$

In order to use the proof of Chapter 4 to get (2.79), we make the change of variable $t \rightarrow \tilde{t}^{-1 / m}$, then it suffices to prove that

$$
\left(\int_{\mathbb{R}^{2}} \int_{2^{-2 m}}^{2^{m}}\left|\tilde{F}_{\lambda}^{\delta^{\prime}} f\left(y, \tilde{t}^{-1 / m}\right)\right|^{4} d y d \tilde{t}\right)^{1 / 4} \leq C \lambda^{1 / 8+\epsilon_{1}} \delta^{\prime-\left(1 / 2+\epsilon_{2}\right)}\|f\|_{L^{4}\left(\mathbb{R}^{2}\right)},
$$

where

$$
\tilde{F}_{\lambda}^{\delta^{\prime}} f\left(y, \tilde{t}^{-1 / m}\right)=\rho_{1}\left(y, \tilde{t}^{-1 / m}\right) \int_{\mathbb{R}^{2}} e^{i\left(\xi \cdot y-\tilde{t}^{-2 / m} \xi_{2} \tilde{\Phi}(\tilde{s}, \delta)\right)} a\left(\xi, \tilde{t}^{-1 / m}\right) \rho_{0}\left(\frac{\delta^{\prime}}{\lambda}|\xi|\right) \tilde{\chi}\left(\frac{\xi_{1}}{\xi_{2}}\right) \hat{f}(\xi) d \xi,
$$

and

$$
\tilde{s}=-\tilde{t}^{1 / m} \frac{\xi_{1}}{\xi_{2}}, \quad-\tilde{t}^{-2 / m} \xi_{2} \tilde{\Phi}(\tilde{s}, \delta)=\frac{\xi_{1}^{2}}{2 \xi_{2}}+\tilde{t}^{\prime}(-1)^{m+1} \frac{\phi^{(m)}(0)}{m !} \cdot \frac{\xi_{1}^{m+2}}{\xi_{2}^{m+1}}+R\left(\tilde{t}^{-1 / m}, \xi, \delta\right) .
$$

Hence, we employ the similar idea of Chapter 4 to obtain (2.80), then Theorem 2.3.6 is proved.

Based on the above theorem, we get that

$$
\begin{aligned}
\left\|\widetilde{M_{j}^{k, 1}} f\right\|_{L^{4}} & \leq C\left(\frac{\delta^{\prime 2}}{\lambda}\right)^{1 / 4} \lambda^{1 / 8+\epsilon_{1}} \delta^{\prime-\left(1 / 2+\epsilon_{2}\right)}\|f\|_{L^{4}} \\
& =C 2^{j^{\prime}\left(-1 / 8+\epsilon_{1}\right)} 2^{k m \epsilon_{2}}\|f\|_{L^{4}} \\
& =C 2^{j\left(-1 / 8+\epsilon_{1}\right)} 2^{k m\left(1 / 8+\epsilon_{2}-\epsilon_{1}\right)}\|f\|_{L^{4}} .
\end{aligned}
$$

Employing the M. Riesz interpolation theorem between (2.78) for $p=2$ and (2.82), we have for $2<p \leq 4$,

$$
\left\|\widetilde{M_{j}^{k, 1}} f\right\|_{L^{p}\left(\mathbb{R}^{3}\right)} \leq C 2^{-j\left(1 / 8-\epsilon_{1}\right)(2-4 / p)} 2^{k m\left[3 / 4-5 /(2 p)+(2-4 / p)\left(\epsilon_{2}-\epsilon_{1}\right)\right]}\|f\|_{L^{p}\left(\mathbb{R}^{2}\right)} .
$$

If $3 / 4-5 /(2 p)+(2-4 / p)\left(\epsilon_{2}-\epsilon_{1}\right) \leq 0$, then (2.76) will follow directly for $j>9 \mathrm{~km}$. If $3 / 4-5 /(2 p)+(2-4 / p)\left(\epsilon_{2}-\epsilon_{1}\right)>0$, then $k m<j / 9$ yields that

$$
\begin{aligned}
\left\|\widetilde{M_{j}^{k, 1}} f\right\|_{L^{p}\left(\mathbb{R}^{3}\right)} & \leq C 2^{-j\left(1 / 8-\epsilon_{1}\right)(2-4 / p)} 2^{j\left[3 / 4-5 /(2 p)+(2-4 / p)\left(\epsilon_{2}-\epsilon_{1}\right)\right] / 9}\|f\|_{L^{p}\left(\mathbb{R}^{2}\right)} \\
& =C 2^{j\left[-1 / 6+2 /(9 p)+(2-4 / p)\left(8 \epsilon_{1}+\epsilon_{2}\right) / 9\right]}\|f\|_{L^{p}\left(\mathbb{R}^{2}\right)} .
\end{aligned}
$$


Employing the M. Riesz interpolation theorem (2.78) for $p=\infty$ and (2.82), we have for $4 \leq p<\infty$,

$$
\left\|\widetilde{M_{j}^{k, 1}} f\right\|_{L^{p}\left(\mathbb{R}^{3}\right)} \leq C 2^{4 j\left(-1 / 8+\epsilon_{1}\right) / p} 2^{4 k m\left(1 / 8+\left(\epsilon_{2}-\epsilon_{1}\right)\right) / p}\|f\|_{L^{p}\left(\mathbb{R}^{2}\right)} .
$$

Since $1 / 8+\left(\epsilon_{2}-\epsilon_{1}\right)>0$, then $k m<j / 9$ implies that

$$
\begin{aligned}
\left\|\widetilde{M_{j}^{k, 1}} f\right\|_{L^{p}\left(\mathbb{R}^{3}\right)} & \leq C 2^{4 j\left(-1 / 8+\epsilon_{1}\right) / p} 2^{4 j\left(1 / 8+\left(\epsilon_{2}-\epsilon_{1}\right)\right) /(9 p)}\|f\|_{L^{p}\left(\mathbb{R}^{2}\right)} \\
& =C 2^{4 j\left(-1+8 \epsilon_{1}+\epsilon_{2}\right) /(9 p)}\|f\|_{L^{p}\left(\mathbb{R}^{2}\right)}
\end{aligned}
$$

which finishes the proof of (2.76), hence of Theorem 2.1.1 when $m>1$.

\subsection{The proof for curves of finite type}

In this section, we will prove Theorem 2.1.3.

The proof of Theorem 2.1.1 inspires us to prove Theorem 2.1.3 directly. Because at the beginning of the proof, we employ a dyadic decomposition to restrict on an interval far away from the origin, and this means whether $d=2$, the curve has still non-vanishing Gaussian curvature in this constant interval and we can still apply the method of stationary phase. We proceed as in the proof of Theorem 2.1.1.

We choose $\tilde{\rho} \in C_{0}^{\infty}(\mathbb{R})$ such that supp $\tilde{\rho} \subset\{x: B / 2 \leq|x| \leq 2 B\}$ and $\sum_{k} \tilde{\rho}\left(2^{k} x\right)=1$. Since the support of $\eta$ is sufficiently small, then we can choose $k$ sufficiently large.

Put

$$
A_{t} f(y):=\int_{\mathbb{R}} f\left(y_{1}-t x, y_{2}-t^{d} x^{d} \phi(x)\right) \eta(x) d x=\sum_{k} \widetilde{A_{t}^{k}} f(y),
$$

where $\widetilde{A_{t}^{k}} f(y):=\int_{\mathbb{R}} f\left(y_{1}-t x, y_{2}-t^{d} x^{d} \phi(x)\right) \eta(x) \tilde{\rho}\left(2^{k} x\right) d x$.

Consider the isometric operator on $L^{p}\left(\mathbb{R}^{2}\right)$ defined by

$$
T f\left(x_{1}, x_{2}\right)=2^{(d+1) k / p} f\left(2^{k} x_{1}, 2^{d k} x_{2}\right) .
$$

As the argument in the last section, it suffices to prove the following estimate

$$
\sum_{k} 2^{-k}\left\|\sup _{t>0}\left|A_{t}^{k}\right|\right\|_{L^{p}} \leq C_{p}, \text { for } p>2
$$

where $A_{t}^{k} f(y):=\int_{\mathbb{R}} f\left(y_{1}-t x, y_{2}-t^{d} x^{d} \phi\left(\frac{x}{2^{k}}\right)\right) \tilde{\rho}(x) \eta\left(2^{-k} x\right) d x$.

By means of the Fourier inversion formula, we can write

$$
A_{t}^{k} f(y)=\frac{1}{(2 \pi)^{2}} \int_{\mathbb{R}^{2}} e^{i \xi \cdot y} \widehat{d \mu_{k, d, m}}\left(\delta_{t} \xi\right) \hat{f}(\xi) d \xi
$$


where

$$
\widehat{d \mu_{k, d, m}}\left(\delta_{t} \xi\right)=\int_{\mathbb{R}} e^{-i\left(t \xi_{1} x+t^{d} \xi_{2} x^{d} \phi\left(\frac{x}{2^{k}}\right)\right)} \tilde{\rho}(x) \eta\left(2^{-k} x\right) d x .
$$

Choosing a non-negative function $\beta \in C_{0}^{\infty}(\mathbb{R})$ as before, set

$$
A_{t, j}^{k} f(y):=\frac{1}{(2 \pi)^{2}} \int_{\mathbb{R}^{2}} e^{i \xi \cdot y} \widehat{d \mu_{k, d, m}}\left(\delta_{t} \xi\right) \beta\left(2^{-j}\left|\delta_{t} \xi\right|\right) \hat{f}(\xi) d \xi
$$

and denote by $M_{j}^{k}$ the corresponding maximal operator.

Since $A_{t, j}^{k}$ is localized to frequencies $\left|\delta_{t} \xi\right| \approx 2^{j}$, we can still use Lemma 2.2.4 to prove that

$$
\left\|M_{j}^{k}\right\|_{L^{p} \rightarrow L^{p}} \lesssim\left\|M_{j, l o c}^{k}\right\|_{L^{p} \rightarrow L^{p}},
$$

where $M_{j, l o c}^{k} f(y):=\sup _{t \in[1,2]}\left|A_{t, j}^{k} f(y)\right|$.

Set

$$
\delta:=2^{-k}, \quad s:=s(\xi, t)=-\frac{\xi_{1}}{t^{d-1} \xi_{2}}, \text { for } \xi_{2} \neq 0
$$

and

$$
\Phi(s, x, \delta)=-s x+x^{d} \phi(\delta x) .
$$

From the proof of Theorem 2.1.1, for fixed $t \in[1,2]$, we would mainly estimate

$$
A_{t, j}^{k} f(y):=\int_{\mathbb{R}^{2}} e^{i\left(\xi \cdot y-t^{d} \xi_{2} \tilde{\Phi}(s, \delta)\right)} \chi_{k, d, m}\left(\frac{\xi_{1}}{t^{d-1} \xi_{2}}\right) \frac{A_{k, d, m}\left(\delta_{t} \xi\right)}{\left(1+\left|\delta_{t} \xi\right|\right)^{1 / 2}} \beta\left(2^{-j}\left|\delta_{t} \xi\right|\right) \hat{f}(\xi) d \xi,
$$

where $\chi_{k, d, m}$ is a smooth function supported in the conical region $\left[c_{k, d, m}, \widetilde{c_{k, d, m}}\right]$, for certain non-zero constant $c_{k, d, m}$ and $\widetilde{c_{k, d, m}}$ dependent only on $k, m$ and $d . A_{k, d, m}$ is a symbol of order zero in $\xi$ and $\left\{A_{k, d, m}\left(\delta_{t} \xi\right)\right\}_{k}$ is contained in a bounded subset of symbol of order zero. Let $\phi(0)=1 / d$ and $\tilde{\Phi}(s, \delta):=\Phi(s, \tilde{q}(s, \delta), \delta)$, then the phase function can be written as

$$
-t^{d} \xi_{2} \tilde{\Phi}(s, \delta)=\left(\frac{1}{d^{\frac{d}{d-1}}}-\frac{1}{d^{\frac{1}{d-1}}}\right)\left(-\frac{d \xi_{1}^{d}}{\xi_{2}}\right)^{\frac{1}{d-1}}-\frac{\delta^{m} \phi^{(m)}(0)}{t^{m} m !}\left(-\frac{\xi_{1}}{\xi_{2}^{\frac{m+1}{m+d}}}\right)^{\frac{d+m}{d-1}}+R(t, \xi, \delta, d),
$$

where $R(t, \xi, \delta, d)$ is homogeneous of degree one in $\xi$ and has at least $m+1$ power of $\delta$. The similar argument with (2.46) allows us to choose $\rho_{1}(y, t) \in C_{0}^{\infty}\left(\mathbb{R}^{2} \times[1 / 2,4]\right)$ and it is sufficient to prove that

$$
\sum_{k} 2^{-k} \sum_{j \geq 1}\left\|\widetilde{M_{j}^{k, 1}}\right\|_{L^{p} \rightarrow L^{p}} \leq C_{p}
$$

where

$$
\widetilde{M_{j}^{k, 1}} f(y):=\sup _{t \in[1,2]}\left|\rho_{1}(y, t) A_{t, j}^{k} f(y)\right| .
$$


Moreover,

$$
\frac{\partial}{\partial t}\left(\rho_{1}(y, t) A_{t, j}^{k} f(y)\right)=\int_{\mathbb{R}^{2}} e^{i\left(\xi \cdot y-t^{d} \xi_{2} \tilde{\Phi}(s, \delta)\right)} h_{k, m, d}(y, t, \xi, j) \hat{f}(\xi) d \xi,
$$

where $\left|h_{k, m}(y, t, \xi, j)\right| \lesssim 2^{j / 2} \delta^{m}+2^{-j / 2}$, which follows from the similar argument with $h_{k}$ in (2.50), together with the facts that

$$
\left|\frac{\partial}{\partial t}\left(-t^{2} \xi_{2} \tilde{\Phi}(s, \delta)\right)\right|=\left|m \frac{\delta^{m} \phi^{(m)}(0)}{t^{m+1} m !}\left(-\frac{\xi_{1}}{\xi_{2}^{\frac{m+1}{m+d}}}\right)^{\frac{d+m}{d-1}}+\frac{\partial}{\partial t} R(t, \xi, \delta, d)\right| \lesssim \delta^{m} 2^{j} .
$$

So we still have the same regularity estimates as (2.3.5) and

$$
\sum_{k} 2^{-k} \sum_{0<j \leq 9 k m} \widetilde{\| M_{j}^{k, 1}} \|_{L^{p} \rightarrow L^{p}} \leq C_{p}, \text { for } 2<p<\infty
$$

Based on these arguments, for $j>9 k m$, let $j=k m+j^{\prime}$ and $\delta^{\prime}=\delta^{m}$, then $j^{\prime}>8 k m$ and $\lambda=2^{j^{\prime}}>\delta^{\prime-8}$. We would be done if we could prove the following theorem.

\section{Theorem 2.4.1.}

$$
\left(\int_{\mathbb{R}^{2}} \int_{2^{-2 m}}^{2^{m}}\left|\tilde{F}_{\lambda}^{\delta^{\prime}} f\left(y, \tilde{t}^{-1 / m}\right)\right|^{4} d y d \tilde{t}\right)^{1 / 4} \leq C \lambda^{1 / 8+\epsilon_{1}} \delta^{\prime-\left(1 / 2+\epsilon_{2}\right)}\|f\|_{L^{4}\left(\mathbb{R}^{2}\right)},
$$

where

$$
\tilde{F}_{\lambda}^{\delta^{\prime}} f\left(y, \tilde{t}^{-1 / m}\right)=\rho_{1}\left(y, \tilde{t}^{-1 / m}\right) \int_{\mathbb{R}^{2}} e^{i\left(\xi \cdot y-\tilde{t}^{-d / m} \xi_{2} \tilde{\Phi}(\tilde{s}, \delta)\right)} a\left(\xi, \tilde{t}^{-1 / m}\right) \rho_{0}\left(\frac{\delta^{\prime}}{\lambda}|\xi|\right) \tilde{\chi}\left(\frac{\xi_{1}}{\xi_{2}}\right) \hat{f}(\xi) d \xi
$$

and

$$
\begin{gathered}
\tilde{s}=-\tilde{t}^{(d-1) / m} \frac{\xi_{1}}{\xi_{2}} \\
-\tilde{t}^{-d / m} \xi_{2} \tilde{\Phi}(\tilde{s}, \delta)=\left(\frac{1}{d^{\frac{d}{d-1}}}-\frac{1}{d^{\frac{1}{d-1}}}\right)\left(-\frac{d \xi_{1}^{d}}{\xi_{2}}\right)^{\frac{1}{d-1}}-\tilde{t} \delta^{\prime} \frac{\phi^{(m)}(0)}{m !}\left(-\frac{\xi_{1}}{\xi_{2}^{\frac{m+1}{m+d}}}\right)^{\frac{d+m}{d-1}}+R\left(\tilde{t}^{-1 / m}, \xi, \delta, d\right) .
\end{gathered}
$$

The proof is similar to the one in Chapter 4. 



\section{Chapter 3}

\section{A model operator}

In this chapter, we will prove Theorem 2.3.4, which will be obtained in a similar way as the proof of $L^{4}$-boundedness of the following operator:

$$
\mathcal{F}_{\lambda}^{\delta} f(y, t)=\rho_{1}(y, t) \int_{\mathbb{R}^{2}} e^{i\left(y \cdot \xi+E(\xi)+t q_{\delta}(\xi)\right)} a(\xi, t) \rho_{0}\left(\frac{\delta}{\lambda}|\xi|\right) \tilde{\chi}\left(\frac{\xi_{1}}{\xi_{2}}\right) \hat{f}(\xi) d \xi,
$$

where $E(\xi)=\frac{\xi_{1}^{2}}{\xi_{2}}, q_{\delta}(\xi)=\delta \cdot \frac{\xi_{1}^{3}}{\xi_{2}^{2}}, \tilde{\chi} \in C_{0}^{\infty}\left(\left[c_{1}, c_{2}\right]\right)\left(c_{1}, c_{2}\right.$ are very small positive constants), $\rho_{0} \in C_{0}^{\infty}((-10,10))$ and $a$ is a symbol of order zero. So we need to prove that for all $\lambda>1 / \delta$,

$$
\left\|\mathcal{F}_{\lambda}^{\delta} f(y, t)\right\|_{L^{4}\left(\mathbb{R}^{3}\right)} \leq C \lambda^{1 / 8+\epsilon_{1}} \delta^{-\left(1 / 2+\epsilon_{2}\right)}\|f\|_{L^{4}\left(\mathbb{R}^{2}\right)} .
$$

The following approach follows the proof of Proposition 3 in [25], but we need various modifications.

We may assume that the $\xi$-support of the symbol $a$ is in the first quadrant. We rewrite

$$
\begin{aligned}
\mathcal{F}_{\lambda}^{\delta} f(y, t) & =\int_{\mathbb{R}} e^{i t \tau} \mathcal{F}_{\lambda}^{\delta} f(y, \hat{\tau}) d \tau \\
& =\int_{\mathbb{R}} \int_{\mathbb{R}^{2}} e^{i(y \cdot \xi+t \tau)} e^{i E(\xi)}\left(\rho_{1}(y, \cdot) a(\xi, \cdot)\right)^{\wedge}\left(\tau-q_{\delta}(\xi)\right) \rho_{0}\left(\frac{\delta}{\lambda}|\xi|\right) \tilde{\chi}\left(\frac{\xi_{1}}{\xi_{2}}\right) \hat{f}(\xi) d \xi d \tau .
\end{aligned}
$$

We can reduce our proof to the situation $\left|\tau-q_{\delta}(\xi)\right| \leq C_{0} \lambda$ for an appropriate constant $C_{0}$ which is small and satisfies $q_{\delta}(\xi)-2 C_{0} \lambda>0$. In fact, if $\left|\tau-q_{\delta}(\xi)\right| \geq C_{0} \lambda$, then

(i) if $\tau \gg q_{\delta}(\xi) \approx \lambda>1$, by $|\xi| \approx \lambda / \delta$, then

$$
\left|\tau-q_{\delta}(\xi)\right| \geq C \tau \geq C(\lambda+|(\delta \xi, \tau)|) ;
$$

(ii) if $\tau \lesssim q_{\delta}(\xi) \approx \lambda>1$, by assumption, then

$$
\left|\tau-q_{\delta}(\xi)\right| \geq C_{0} \lambda \geq C(\lambda+|(\delta \xi, \tau)|) .
$$


Define the operator $\mathcal{L}=I-\triangle_{\xi, \tau}$, where $\triangle_{\xi, \tau}=\frac{\partial^{2}}{\partial \xi_{1}^{2}}+\frac{\partial^{2}}{\partial \xi_{2}^{2}}+\frac{\partial^{2}}{\partial \tau^{2}}$, then the above arguments of (i) and (ii), $\rho_{1} \in C_{0}^{\infty}\left(\mathbb{R}^{2} \times[1 / 2,4]\right)$, together with the fact that $a$ is a symbol of order zero, imply that for sufficiently large $N^{\prime}$,

$$
\left|\mathcal{L}^{2}\left(\rho_{1}(y, \cdot) a(\cdot, \xi)\right)^{\wedge}\left(\tau-q_{\delta}(\xi)\right)\right| \leq C_{N^{\prime}}(\lambda+|(\delta \xi, \tau)|)^{-N^{\prime}}
$$

and

$$
\begin{aligned}
& \left|\mathcal{L}^{2}\left(e^{i E(\xi)}\left(\rho_{1}(y, \cdot) a(\cdot, \xi)\right)^{\wedge}\left(\tau-q_{\delta}(\xi)\right) \rho_{0}\left(\frac{\delta}{\lambda}|\xi|\right) \tilde{\chi}\left(\frac{\xi_{1}}{\xi_{2}}\right)\right)\right| \\
& \leq C_{N^{\prime}}(\lambda+|(\delta \xi, \tau)|)^{-N^{\prime}} \\
& \leq C_{N^{\prime}} \lambda^{-N^{\prime} / 2}(1+|(\delta \xi, \tau)|)^{-N^{\prime} / 2} .
\end{aligned}
$$

Since $\mathcal{L}^{2}\left(e^{i((y-x) \cdot \xi+t \tau)}\right)=\left(1+|(y-x, t)|^{2}\right)^{2}$, by integration by parts in $(\xi, \tau)$ and the assumption $\left|\tau-q_{\delta}(\xi)\right| \geq C_{0} \lambda$, the kernel $K(y, t ; x)$ of the operator $\mathcal{F}_{\lambda}^{\delta}$ can be controlled by

$$
\begin{aligned}
|K(y, t ; x)| & \leq C_{N^{\prime}} \frac{1}{\left(1+|(y-x, t)|^{2}\right)^{2}} \lambda^{-N^{\prime} / 2} \int_{\mathbb{R}^{3}}(1+|(\delta \xi, \tau)|)^{-N^{\prime} / 2} d \xi d \tau \\
& \leq C_{N^{\prime}} \frac{1}{\left(1+|(y-x, t)|^{2}\right)^{2}} \lambda^{-N^{\prime} / 2} \delta^{-2} .
\end{aligned}
$$

Since $\delta \lambda>1$, then for sufficiently large $N<N^{\prime}$,

$$
|K(y, t ; x)| \leq C_{N} \lambda^{-N} \frac{1}{\left(1+|(y-x, t)|^{2}\right)^{2}} .
$$

In the next steps, we will always assume that $\left|\tau-q_{\delta}(\xi)\right| \leq C_{0} \lambda$ for a small appropriate constant $C_{0}$, which shows that

$$
\lambda \approx q_{\delta}(\xi)-C_{0} \lambda \leq \tau \leq q_{\delta}(\xi)+C_{0} \lambda \approx \lambda .
$$

Let $\varphi \in C_{0}^{\infty}(\mathbb{R})$ satisfy supp $\varphi \subset[-1,1]$ and $\sum_{n \in \mathbb{Z}} \varphi^{2}(\cdot-n)=1$. In order to give a decomposition for $\tau$, the dual to $t$, we define the operator $P^{n}$ on functions in $\mathbb{R}^{3}$ by

$$
\left(P^{n} g\right)^{\wedge}(\eta, \tau)=\varphi\left(\lambda^{-1 / 2} \tau-n\right) \widehat{g}(\eta, \tau) .
$$

Similarly, we define $\widehat{f}_{n}(\xi)=\varphi\left(\left(\lambda^{-1 / 2} q_{\delta}(\xi)-n\right) / 10\right) \hat{f}(\xi)$.

Lemma 3.0.2. For $N>0$ and $1 \leq p \leq \infty$,

$$
\left\|\sum_{n}\left(P^{n}\right)^{2} \mathcal{F}_{\lambda}^{\delta}\left(f-f_{n}\right)\right\|_{L^{p}\left(\mathbb{R}^{3}\right)} \leq C_{N} \lambda^{-N}\|f\|_{L^{p}\left(\mathbb{R}^{2}\right)} .
$$


Proof. For fixed $n$, we have

$$
\begin{aligned}
\left(P^{n}\right)^{2} \mathcal{F}_{\lambda}^{\delta}\left(f-f_{n}\right)(y, t)= & \int_{\mathbb{R}} \int_{\mathbb{R}^{2}} e^{i(y \cdot \xi+t \tau+E(\xi))} \varphi^{2}\left(\lambda^{-1 / 2} \tau-n\right)\left(\rho_{1}(y, \cdot) a(\xi, \cdot)\right)^{\wedge}\left(\tau-q_{\delta}(\xi)\right) \\
& \times \rho_{0}\left(\frac{\delta}{\lambda}|\xi|\right) \tilde{\chi}\left(\frac{\xi_{1}}{\xi_{2}}\right)\left[1-\varphi\left(\left(\lambda^{-1 / 2} q_{\delta}(\xi)-n\right) / 10\right)\right] \hat{f}(\xi) d \xi d \tau
\end{aligned}
$$

From the support of $\varphi$, we have $\lambda^{1 / 2}(n-1) \leq \tau \leq \lambda^{1 / 2}(n+1)$, together with (3.5), we get $n \approx \lambda^{1 / 2}$. We observe that $q_{\delta}(\xi) \notin\left[\lambda^{1 / 2}(n-10), \lambda^{1 / 2}(n+10)\right]$, then we get $\left|\tau-q_{\delta}(\xi)\right| \gtrsim(\lambda+|(\delta \xi, \tau)|)^{1 / 2}$. Finally, as in the estimate (3.4), the kernel of $\left(P^{n}\right)^{2} \mathcal{F}_{\lambda}^{\delta}$ defined by

$$
\begin{gathered}
\int_{\mathbb{R}} \int_{\mathbb{R}^{2}} e^{i[(y-x) \cdot \xi+t \tau+E(\xi)]} \varphi^{2}\left(\lambda^{-1 / 2} \tau-n\right)\left(\rho_{1}(y, \cdot) a(\xi, \cdot)\right)^{\wedge}\left(\tau-q_{\delta}(\xi)\right) \rho_{0}\left(\frac{\delta}{\lambda}|\xi|\right) \tilde{\chi}\left(\frac{\xi_{1}}{\xi_{2}}\right) \\
\times\left[1-\varphi\left(\left(\lambda^{-1 / 2} q_{\delta}(\xi)-n\right) / 10\right)\right] d \xi d \tau
\end{gathered}
$$

can be dominated by $\mathcal{O}\left(\lambda^{-N}\left(1+|(y-x, t)|^{2}\right)^{-2}\right)$ and

$$
\left\|\sum_{n}\left(P^{n}\right)^{2} \mathcal{F}_{\lambda}^{\delta}\left(f-f_{n}\right)\right\|_{L^{p}\left(\mathbb{R}^{3}\right)} \leq C_{N^{\prime}} \sum_{n \approx \lambda^{1 / 2}} \lambda^{-N^{\prime}}\|f\|_{L^{p}\left(\mathbb{R}^{2}\right)} \leq C_{N} \lambda^{-N}\|f\|_{L^{p}\left(\mathbb{R}^{2}\right)} .
$$

Lemma 3.0.3. For $2 \leq p \leq \infty$, we have

$$
\left\|\sum_{n \approx \lambda^{1 / 2}}\left(P^{n}\right)^{2} \mathcal{F}_{\lambda}^{\delta} f_{n}\right\|_{L^{p}\left(\mathbb{R}^{3}\right)} \leq C \lambda^{(1 / 4-1 /(2 p))}\left\|\left(\sum_{n \approx \lambda^{1 / 2}}\left|P^{n} \mathcal{F}_{\lambda}^{\delta} f_{n}\right|^{2}\right)^{1 / 2}\right\|_{L^{p}\left(\mathbb{R}^{3}\right)} .
$$

The idea can be found in [25].

So far we have used a radial decomposition of the Fourier integral operator $\mathcal{F}_{\lambda}^{\delta}$ with respect to frequency variables. Now we make a further angular decomposition. As we have introduced in Lemma 2.3.2, here we redefine the homogeneous partition of unity of $\mathbb{R}^{2} \backslash\{0\}$ that depends on the scale $\lambda / \delta$. Specially, for fixed $j>0$, we choose functions $\chi_{\nu}, \nu=0,1,2, \cdots$, and the size of every angle is $(\delta / \lambda)^{1 / 2}$. Meanwhile $\chi_{\nu}$ satisfies the following conditions:

$$
\begin{gathered}
\sum_{\nu} \chi_{\nu}(\xi)=1 \text { for all } \xi \neq 0 \\
\left|\partial_{\xi}^{\alpha} \chi_{\nu}\right| \leq A_{\alpha}(\lambda / \delta)^{|\alpha| / 2}|\xi|^{-|\alpha|} \text { for all } \xi \neq 0
\end{gathered}
$$

Define $Q_{\nu}$ by

$$
\left(Q_{\nu} g\right)^{\wedge}(\eta, \tau)=\Psi_{\nu}^{\delta}(\eta, \tau) \widehat{g}(\eta, \tau)=\psi\left(\frac{\tau-q_{\delta}(\eta)}{|\tau / \delta|^{\epsilon}}\right) \chi_{\nu}\left(\eta^{\prime}\right) \widehat{g}(\eta, \tau),
$$


where $\eta^{\prime}=\frac{\eta}{|\eta|}$ and $\psi \in C_{0}^{\infty}(\mathbb{R})$ is supported in $[-2,2]$ and equal to 1 in $[-1,1]$. In fact, $\left(Q_{\nu} g\right)^{\wedge}(\eta, \tau)$ is supported in a thin sector intersected with a thin neighborhood of the cone $\left\{\left(\eta, q_{\delta}(\eta)\right)\right\}$, see Figure 2 .

Put

$$
\mathcal{F}_{\lambda, \nu}^{\delta} f(y, t)=\rho_{1}(y, t) \int_{\mathbb{R}^{2}} e^{i\left(y \cdot \xi+E(\xi)+t q_{\delta}(\xi)\right)} a(\xi, t) \tilde{\chi}\left(\frac{\xi_{1}}{\xi_{2}}\right) \rho_{0}\left(\frac{\delta}{\lambda}|\xi|\right) \chi_{\nu}(\xi) \widehat{f}(\xi) d \xi
$$

Lemma 3.0.4. Given a fixed $\epsilon>0$ and $n \approx \lambda^{1 / 2}$, we define

$$
R_{\lambda, \nu}^{\delta} f=P^{n}\left(\mathcal{F}_{\lambda, \nu}^{\delta} f\right)-P^{n} Q_{\nu}\left(\mathcal{F}_{\lambda, \nu}^{\delta} f\right)
$$

For any $N>0$, there is a uniform constant $C_{N}$ so that

$$
\left\|R_{\lambda, \nu}^{\delta} f_{n}\right\|_{L^{p}\left(\mathbb{R}^{3}\right)} \leq C_{N}\left(\frac{\lambda}{\delta}\right)^{-N}\|f\|_{L^{p}\left(\mathbb{R}^{2}\right)}
$$

Proof.

$$
\begin{aligned}
R_{\lambda, \nu}^{\delta} f_{n}(y, t)= & \int_{\mathbb{R}^{2}} e^{i E(\xi)}\left[\int_{\mathbb{R}^{2}} \int_{\mathbb{R}} e^{i(y \cdot \eta+t \tau)}\left(1-\Psi_{\nu}^{\delta}(\eta, \tau)\right) \varphi\left(\lambda^{-1 / 2} \tau-n\right)\right. \\
& \left.\times\left(\rho_{1} \cdot a(\xi, \cdot)\right)^{\wedge}\left(\eta-\xi, \tau-q_{\delta}(\xi)\right) d \eta d \tau\right] \rho_{0}\left(\frac{\delta}{\lambda}|\xi|\right) \tilde{\chi}\left(\frac{\xi_{1}}{\xi_{2}}\right) \chi_{\nu}(\xi) \widehat{f_{n}}(\xi) d \xi
\end{aligned}
$$

In order to estimate $\left|\left(\eta-\xi, \tau-q_{\delta}(\xi)\right)\right|$, we split our consideration into different cases: 1. When $\eta^{\prime} \notin \operatorname{supp} \chi_{\nu}$, but $\xi \in \operatorname{supp} \chi_{\nu}$, then $|\eta-\xi| \geq C\left(\frac{\lambda}{\delta}\right)^{1 / 2}$ and the following inequalities will hold true:

(1). if $|\eta| \gg|\xi| \approx \frac{\lambda}{\delta}$

$$
|\eta-\xi| \geq C|\eta| \geq C\left(\frac{\lambda}{\delta}+\left|\left(\eta, \frac{\tau}{\delta}\right)\right|\right)
$$

(2). if $|\eta| \lesssim|\xi| \approx \frac{\lambda}{\delta}$

$$
|\eta-\xi| \geq C\left(\frac{\lambda}{\delta}\right)^{1 / 2} \geq C\left(\frac{\lambda}{\delta}+\left|\left(\eta, \frac{\tau}{\delta}\right)\right|\right)^{1 / 2}
$$

2. When $\eta^{\prime} \in \operatorname{supp} \chi_{\nu}$, but $\frac{\left|\tau-q_{\delta}(\eta)\right|}{|\tau / \delta|^{\epsilon}} \geq 2$ and $\xi \in \operatorname{supp} \chi_{\nu}$, then the following inequalities will hold true:

(1). if $|\eta| \gg|\xi| \approx \frac{\lambda}{\delta}$

$$
|\eta-\xi| \geq C|\eta| \geq C\left(\frac{\lambda}{\delta}+\left|\left(\eta, \frac{\tau}{\delta}\right)\right|\right)
$$

(2). if $|\eta| \lesssim|\xi| \approx \frac{\lambda}{\delta}$, for $\mu \ll \epsilon$, we consider two subcases: 
a. when $|\eta-\xi| \geq C|\tau / \delta|^{\mu}$, we have

$$
|\eta-\xi| \geq C\left(\frac{\lambda}{\delta}+\left|\left(\eta, \frac{\tau}{\delta}\right)\right|\right)^{\mu},
$$

b. when $|\eta-\xi| \leq C|\tau / \delta|^{\mu}$, since

$$
\left|\tau-q_{\delta}(\xi)\right| \geq\left|\tau-q_{\delta}(\eta)\right|-\left|q_{\delta}(\xi)-q_{\delta}(\eta)\right|
$$

and

then we have

$$
\left|q_{\delta}(\xi)-q_{\delta}(\eta)\right| \approx \delta|\xi-\eta| \leq C \delta\left|\frac{\tau}{\delta}\right|^{\mu}
$$

$$
\left|\tau-q_{\delta}(\xi)\right| \geq 2\left|\frac{\tau}{\delta}\right|^{\epsilon}-C \delta\left|\frac{\tau}{\delta}\right|^{\mu} \approx\left(\frac{\lambda}{\delta}+\left|\left(\eta, \frac{\tau}{\delta}\right)\right|\right)^{\epsilon} .
$$

Combing all the cases, if we set $\mathcal{L}=I-\triangle_{\eta, \tau}$, for any $2<M \ll \mu N^{\prime}$, we obtain,

$$
\left|\mathcal{L}^{M}\left(\widehat{\rho}_{1}\left(\eta-\xi, \tau-q_{\delta}(\xi)\right)\right)\right| \leq C_{N^{\prime}}\left(\frac{\lambda}{\delta}+\left|\left(\eta, \frac{\tau}{\delta}\right)\right|\right)^{-\mu N^{\prime}} .
$$

The estimate (3.9) implies that $\left|\chi_{\nu}\left(\eta^{\prime}\right)\right| \leq(\lambda / \delta)^{1 / 2}$, in addition to (3.12), we get

$\mathcal{L}^{M}\left(\left(1-\Psi_{\nu}^{\delta}(\eta, \tau)\right) \varphi\left(\lambda^{-1 / 2} \tau-n\right) \widehat{\rho}_{1}\left(\eta-\xi, \tau-q_{\delta}(\xi)\right)\right) \leq C_{M, N^{\prime}}\left(\frac{\lambda}{\delta}\right)^{M / 2}\left(\frac{\lambda}{\delta}+\left|\left(\eta, \frac{\tau}{\delta}\right)\right|\right)^{-\mu N^{\prime}}$.

By integration by parts for $(\eta, \tau)$, we have

$$
\begin{aligned}
\mid & R_{\lambda, \nu}^{\delta} f_{n}(y, t) \mid \\
\leq & \int_{\mathbb{R}^{2}}\left|\int_{\mathbb{R}^{2}} \int_{\mathbb{R}} e^{i(y \cdot \eta+t \tau)}\left(1-\Psi_{\nu}^{\delta}(\eta, \tau)\right) \varphi\left(\lambda^{-1 / 2} \tau-n\right)\left(\rho_{1} \cdot a(\xi, \cdot)\right)^{\wedge}\left(\eta-\xi, \tau-q_{\delta}(\xi)\right) d \eta d \tau\right| \\
& \times\left|\rho_{0}\left(\frac{\delta}{\lambda}|\xi|\right) \tilde{\chi}\left(\frac{\xi_{1}}{\xi_{2}}\right) \chi_{\nu}(\xi) \widehat{f_{n}}(\xi)\right| d \xi \\
\leq & \int_{\mathbb{R}^{2}} \int_{\mathbb{R}^{2}} \int_{\mathbb{R}}\left|\mathcal{L}^{M}\left(\left(1-\Psi_{\nu}^{\delta}(\eta, \tau)\right) \varphi\left(\lambda^{-1 / 2} \tau-n\right)\left(\rho_{1} \cdot a(\xi, \cdot)\right)^{\wedge}\left(\eta-\xi, \tau-q_{\delta}(\xi)\right)\right)\right| d \eta d \tau \\
& \times \frac{1}{\left(1+|(y, t)|^{2}\right)^{M}}\left|\rho_{0}\left(\frac{\delta}{\lambda}|\xi|\right) \tilde{\chi}\left(\frac{\xi_{1}}{\xi_{2}}\right) \chi_{\nu}(\xi) \widehat{f_{n}}(\xi)\right| d \xi \\
\leq & \frac{C_{M, N^{\prime}}}{\left(1+|(y, t)|^{2}\right)^{M}} \int_{\mathbb{R}^{2}} \int_{\mathbb{R}^{2}} \int_{\mathbb{R}}\left(\frac{\lambda}{\delta}\right)^{M}\left(\frac{\lambda}{\delta}+\left|\left(\eta, \frac{\tau}{\delta}\right)\right|\right)^{-\mu N^{\prime}} d \eta d \tau\left|\rho_{0}\left(\frac{\delta}{\lambda}|\xi|\right) \tilde{\chi}\left(\frac{\xi_{1}}{\xi_{2}}\right) \chi_{\nu}(\xi) \widehat{f_{n}}(\xi)\right| d \xi \\
\leq & C_{M, N^{\prime}}\left(\frac{\lambda}{\delta}\right)^{M-\mu N^{\prime} / 2} \frac{\delta}{\left(1+|(y, t)|^{2}\right)^{M}} \int_{\mathbb{R}^{2}}\left|\rho_{0}\left(\frac{\delta}{\lambda}|\xi|\right) \tilde{\chi}\left(\frac{\xi_{1}}{\xi_{2}}\right) \chi_{\nu}(\xi) \varphi\left(\frac{\lambda^{-1 / 2} q_{\delta}(\xi)-n}{10}\right)\right| d \xi\|\widehat{f}\|_{L^{\infty}} \\
\leq & C_{M, N^{\prime}} \delta \cdot \frac{\lambda}{\delta^{3 / 2}}\left(\frac{\lambda}{\delta}\right)^{M-\mu N^{\prime} / 2} \frac{1}{\left(1+|(y, t)|^{2}\right)^{M}}\|\widehat{f}\|_{L^{\infty}} .
\end{aligned}
$$


The last inequality follows from the volume of set $\left\{\xi: \rho_{0}\left(\frac{\delta}{\lambda}|\xi|\right) \tilde{\chi}\left(\frac{\xi_{1}}{\xi_{2}}\right) \chi_{\nu}(\xi) \neq 0\right\}$.

Since we have supposed that $f$ is supported in a fixed compact set, then there exists a sufficiently large $C_{M, N^{\prime}, p}$ so that the following inequality holds true,

$$
\left|R_{\lambda, \nu}^{\delta} f_{n}(y, t)\right| \leq C_{M, N^{\prime}, p}\left(\frac{\lambda}{\delta}\right)^{-N} \frac{1}{\left(1+|(y, t)|^{2}\right)^{M}}\|f\|_{L^{p}}
$$

We have finished the proof of Lemma 3.0.4.

Until now, since $\sum_{n}\left(P^{n}\right)^{2}$ is the identity, applying Lemma 3.0.2, then we get

$$
\begin{aligned}
\left\|\mathcal{F}_{\lambda}^{\delta} f\right\|_{L^{4}} & =\left\|\sum_{n}\left(P^{n}\right)^{2} \mathcal{F}_{\lambda}^{\delta} f\right\|_{L^{4}} \\
& \leq\left\|\sum_{n}\left(P^{n}\right)^{2} \mathcal{F}_{\lambda}^{\delta} f_{n}\right\|_{L^{4}}+\left\|\sum_{n}\left(P^{n}\right)^{2} \mathcal{F}_{\lambda}^{\delta}\left(f-f_{n}\right)\right\|_{L^{4}} \\
& \leq\left\|\sum_{n}\left(P^{n}\right)^{2} \mathcal{F}_{\lambda}^{\delta} f_{n}\right\|_{L^{4}}+C_{N} \lambda^{-N}\|f\|_{L^{4}} .
\end{aligned}
$$

It is more convenient to consider a related square function than to estimate the sum of the main term in the right-hand side directly, so by Lemma 3.0.3 and Lemma 3.0.4, we obtain

$$
\begin{aligned}
\left\|\sum_{n}\left(P^{n}\right)^{2} \mathcal{F}_{\lambda}^{\delta} f_{n}\right\|_{L^{4}} & \leq C \lambda^{1 / 8}\left\|\left(\sum_{n}\left|P^{n} \mathcal{F}_{\lambda}^{\delta} f_{n}\right|^{2}\right)^{1 / 2}\right\|_{L^{4}} \\
& \leq C \lambda^{1 / 8}\left\|\left(\sum_{n}\left|P^{n} \sum_{\nu} Q_{\nu}\left(\mathcal{F}_{\lambda, \nu}^{\delta} f_{n}\right)\right|^{2}\right)^{1 / 2}\right\|_{L^{4}}+C_{N} \lambda^{-N}\|f\|_{L^{4}}
\end{aligned}
$$

To estimate the main term in the last expression we notice that, for each fixed $n \approx \lambda^{1 / 2}$ and $\nu$, the Fourier transform of $P^{n} Q_{\nu} g$ is supported in the following region (see Figure 2),

$$
\mathcal{U}_{\nu}^{n}=\left\{(\eta, \tau) \in \mathbb{R}^{3}:(\eta, \tau) \in \operatorname{supp} \Psi_{\nu}^{\delta}(\eta, \tau),\left|\lambda^{-1 / 2} \tau-n\right| \leq 1\right\}
$$

We need an estimate of the number of overlaps of algebraic sums of the sets $\mathcal{U}_{\nu}^{n}, \mathcal{U}_{\nu^{\prime}}^{n^{\prime}}$, for fixed $n, n^{\prime}$ corresponding to indices $\nu$ for which $\xi_{\nu}$ lie in the first quadrant. 


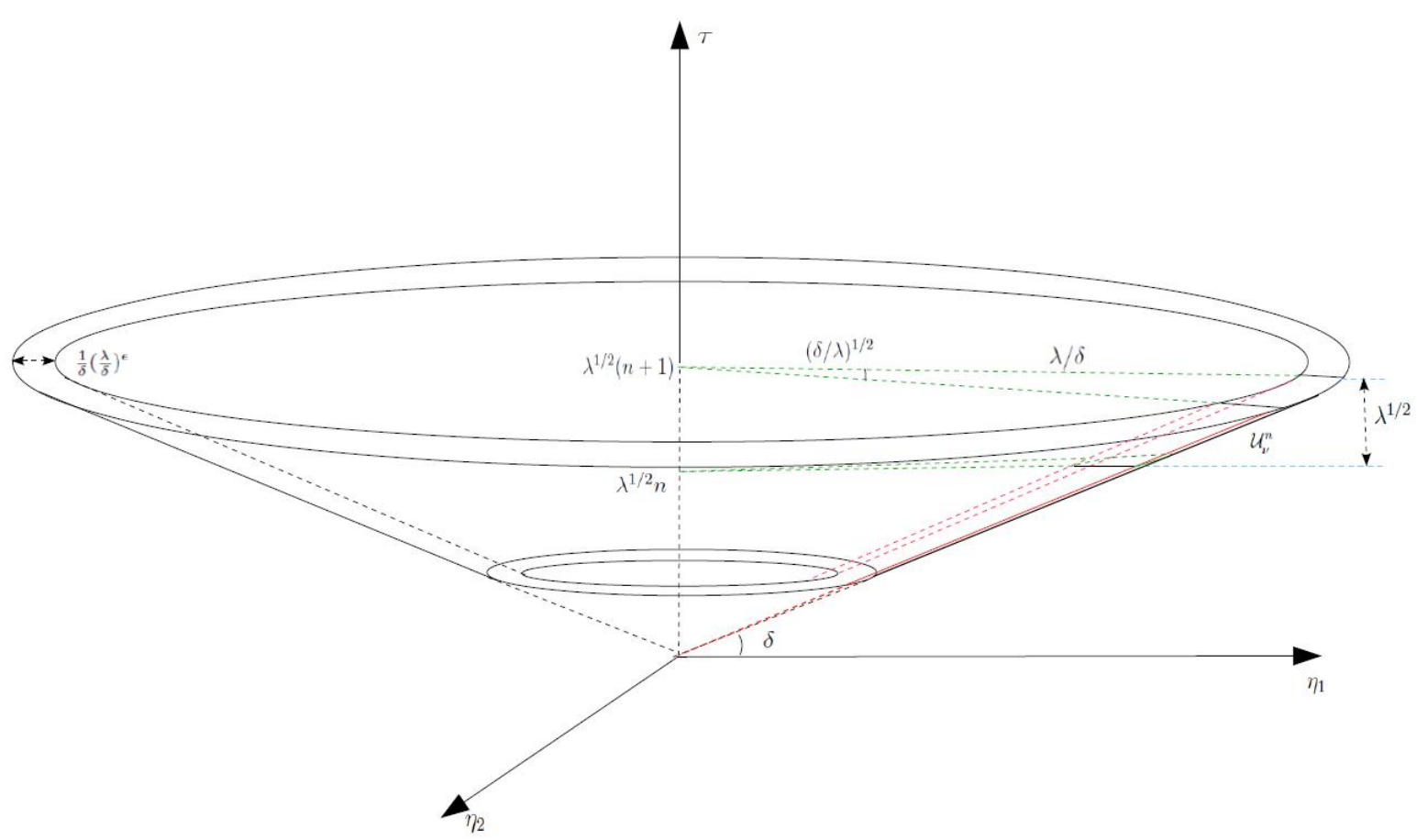

Figure 2: A thin neighborhood of the cone $\tau=\delta|\eta|$ and $\mathcal{U}_{\nu}^{n}$

( The figure of the cone $\tau=q_{\delta}(\eta)$ is similar to a sector of the cone $\tau=\delta|\eta|$. )

Lemma 3.0.5. For fixed $n, n^{\prime} \in \mathbb{Z}$, there is a constant $C$, independent of $n, n^{\prime}, \lambda$ and $\delta$, such that

$$
\sum_{\nu, \nu^{\prime}} \chi_{\mathcal{U}_{\nu}^{n}+\mathcal{U}_{\nu^{\prime}}^{n^{\prime}}}(\eta, \tau) \leq C\left(\frac{\lambda}{\delta}\right)^{2 \epsilon} \log _{2}\left(\frac{\lambda}{\delta}\right)^{1 / 2}
$$

Proof. First, we split every $\mathcal{U}_{\nu}^{n}$ into $\left(\frac{\lambda}{\delta}\right)^{\epsilon}$ pieces along the normal direction to the cone $\tau=q_{\delta}(\eta)$ (vertical cross-section of $\mathcal{U}_{\nu}^{n}$ will be shown in Figure $3(1)$ ), then it suffices to prove that

$$
\sum_{\nu, \nu^{\prime}} \chi_{\tilde{\mathcal{U}}_{\nu}^{n}+\tilde{\mathcal{U}}_{\nu^{\prime}}^{n^{\prime}}}(\eta, \tau) \leq C \log _{2}\left(\frac{\lambda}{\delta}\right)^{1 / 2}
$$

where $\tilde{\mathcal{U}}_{\nu}^{n}$ is comparable to a rectangle tangential to the cone of dimension $1 \times \frac{\lambda^{1 / 2}}{\delta} \times$ $\left(\frac{\lambda}{\delta}\right)^{1 / 2}$ ( vertical cross-section of $\tilde{\mathcal{U}}_{\nu}^{n}$ and the comparable rectangle will be shown in Figure 3 (2) and the left-hand one of Figure 4). 


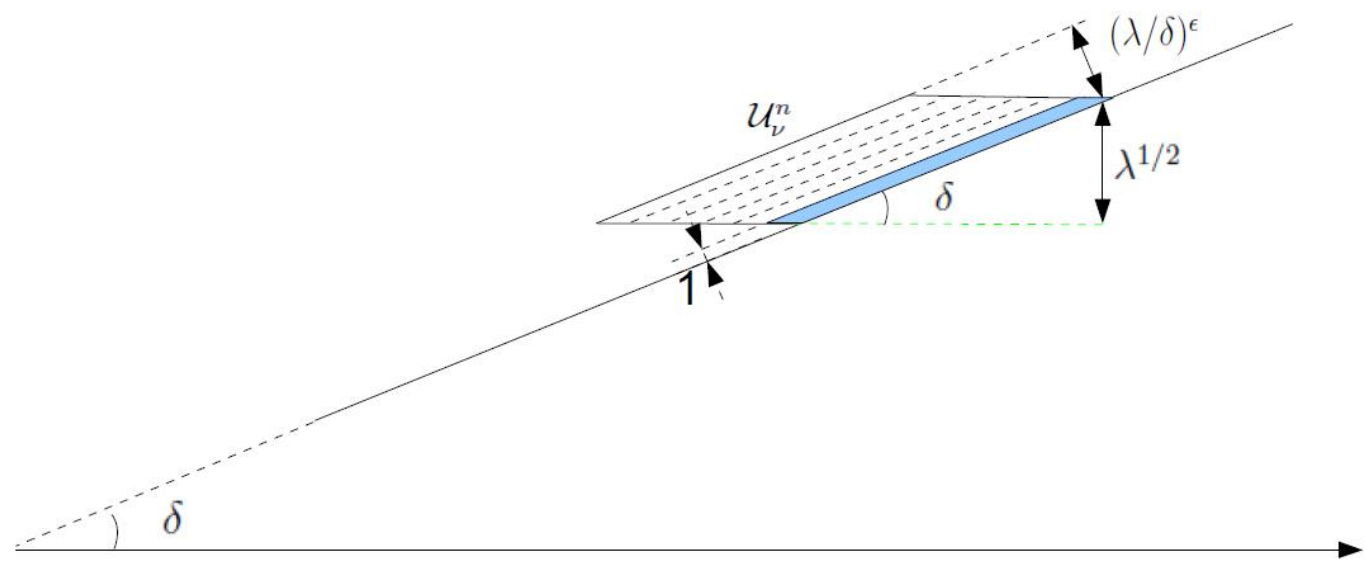

Figure 3 (1): Vertical cross-section of $\mathcal{U}_{\nu}^{n}$

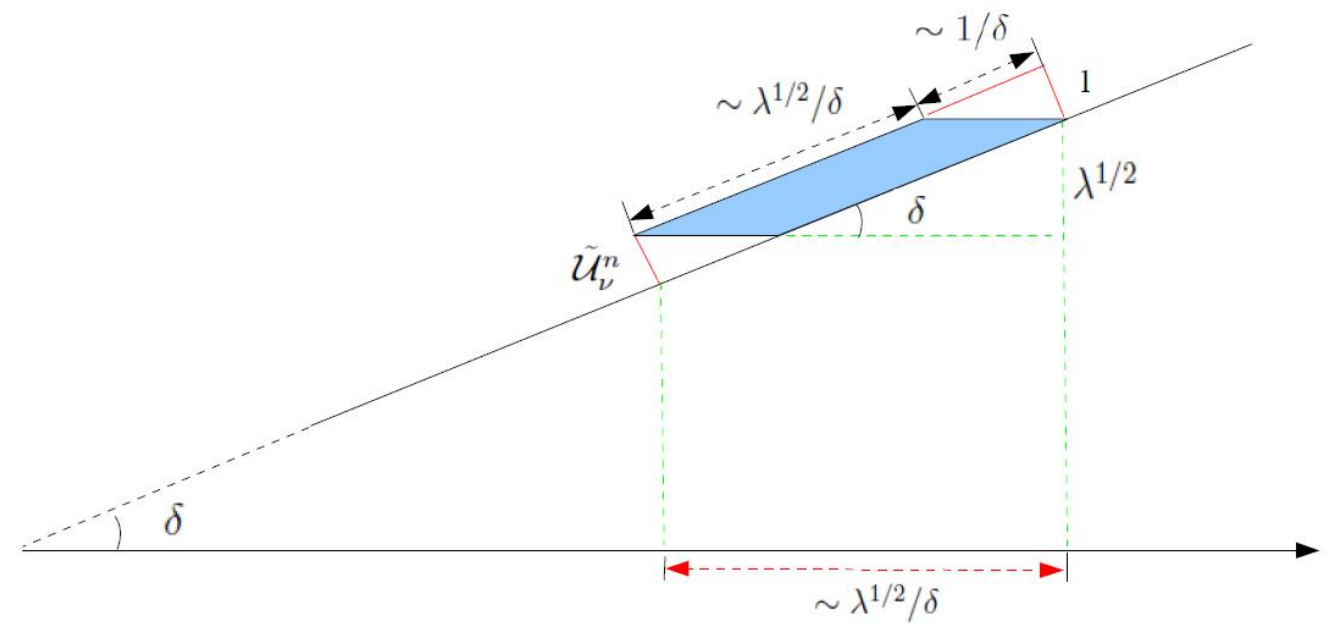

Figure 3 (2): Vertical cross-section of $\tilde{\mathcal{U}}_{\nu}^{n}$ and the comparable rectangle

Furthermore, here we use similar arguments as in [26] to prove a scaled version by $1 / \lambda$ of inequality (3.15).

To this end we define $\Gamma_{\nu}=\left\{\xi \in \mathbb{R}^{2} \backslash 0: \sqrt{\frac{\delta}{\lambda}} \nu \leq\left|\frac{\xi_{2}}{\xi_{1}}\right| \leq \sqrt{\frac{\delta}{\lambda}}(\nu+1)\right.$ for $\nu=\left(\frac{\pi}{2}-\right.$ $\left.c_{2}\right) \sqrt{\frac{\lambda}{\delta}}, \cdots,\left(\frac{\pi}{2}-c_{1}\right) \sqrt{\frac{\lambda}{\delta}}$ and here non-zero positive numbers $c_{1}, c_{2}$ with $c_{1}<c_{2}$ depend on the support of $\tilde{\chi}$. Next, for $n=0,1, \cdots, \lambda^{1 / 2}$, let $\Lambda_{\nu}^{n}$ be the set of all $(\eta, \tau)$ such that $\operatorname{dist}\left((\eta, \tau),\left(\xi, q_{\delta}(\xi)\right)\right) \leq \lambda^{-1}$ for some $\xi \in \Gamma_{\nu}$ with $q_{\delta}(\xi) \in\left[\left(\frac{\pi}{2}-c_{1}\right)^{-2}+\frac{n}{\sqrt{\lambda}},\left(\frac{\pi}{2}-\right.\right.$ $\left.\left.c_{1}\right)^{-2}+\frac{(n+1)}{\sqrt{\lambda}}\right]$. Thus $\Lambda_{\nu}^{n}$ is basically a $\lambda^{-1} \times \frac{1}{\delta \sqrt{\lambda}} \times \frac{1}{\sqrt{\delta \lambda}}$ rectangle (see the right one of 
Figure 4) lying on $\left\{\left(\xi, q_{\delta}(\xi)\right): q_{\delta}(\xi) \in\left[\left(\frac{\pi}{2}-c_{1}\right)^{-2},\left(\frac{\pi}{2}-c_{2}\right)^{-2}\right]\right\}$. Its shortest side points in the normal direction to the cone.
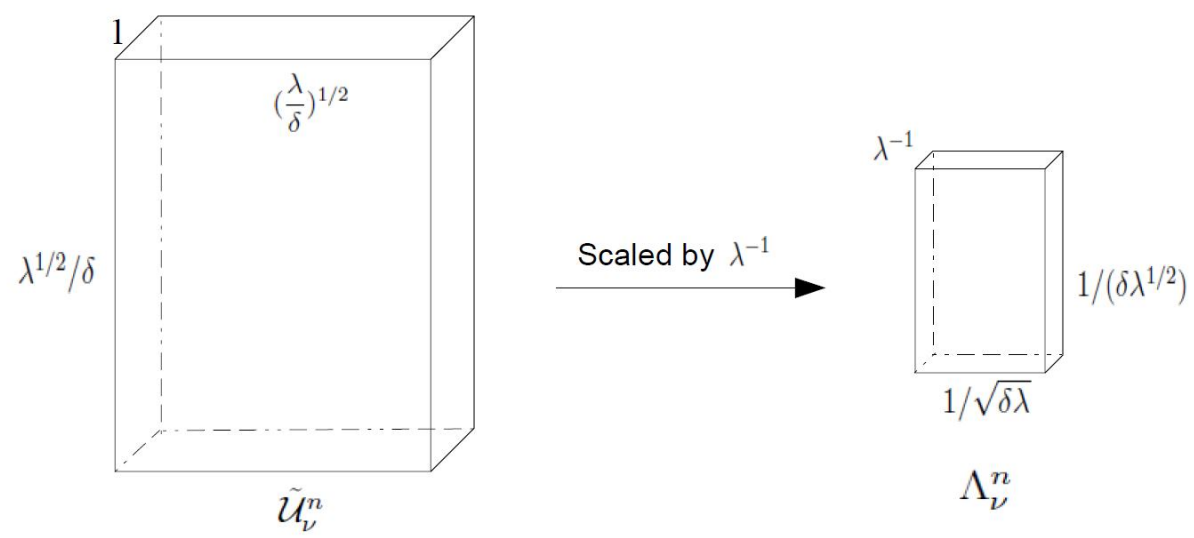

$\Lambda_{\nu}^{n}$

Figure 4

The scaled version of the overlap lemma is that there is a constant $C$ independent of $n, n^{\prime}, \lambda$ and $\delta$ such that

$$
\sum_{\nu, \nu^{\prime}} \chi_{\Lambda_{\nu}^{n}+\Lambda_{\nu^{\prime}}^{n^{\prime}}}(\eta, \tau) \leq C \log _{2}\left(\frac{\lambda}{\delta}\right)^{1 / 2}
$$

However, inequality (3.16) follows from

$$
\sum_{\left|\nu-\nu^{\prime}\right| \approx 2^{\ell}} \chi_{\Lambda_{\nu}^{n}+\Lambda_{\nu^{\prime}}^{n^{\prime}}}(\eta, \tau) \leq C, \quad 0 \leq \ell \leq \log _{2}\left(\frac{\lambda}{\delta}\right)^{1 / 2} .
$$

To deduce inequality (3.17), we give a simple argument to show that for $\bar{\xi} \in \Gamma_{\nu}$ and $\tilde{\xi} \in$ $\Gamma_{\nu^{\prime}}$, the angle between the normals to the cone at $\left(\bar{\xi}, q_{\delta}(\bar{\xi})\right)$ and $\left(\tilde{\xi}, q_{\delta}(\tilde{\xi})\right)$ is $\approx 2^{\ell}\left(\frac{\delta}{\lambda}\right)^{1 / 2}$ if $\left|\nu-\nu^{\prime}\right| \approx 2^{\ell}$. It will follow from the claim that $\operatorname{dist}(P, A) \approx \operatorname{dist}(P, D) \approx 1 / \delta$ (See Figure 5 for $\left.q_{\delta}(\xi)=1\right)$. Next, we will first prove this claim.

In Figure 5, $A E$ and $D E$ are the tangent line of the curve $q_{\delta}(\xi)=1$ at point $\left(\bar{\xi}_{1}, 0\right)$, $\left(\tilde{\xi}_{1}, 0\right)$, where $\bar{\xi}_{1}, \tilde{\xi}_{1} \approx 1 / \delta . P A$ and $P D$ are perpendicular respectively $A E$ and $D E$.

From the expression of the curve $q_{\delta}(\xi)=1$, it is easy to know that $\frac{\pi}{2}-c_{2} \leq \tan \angle A O B \approx$ $\tan \angle B, \tan \angle C \approx \tan \angle D O B \leq \frac{\pi}{2}-c_{1}$.

Since $\tan (\angle D O B-\angle A O B) \approx 2^{\ell}\left(\frac{\delta}{\lambda}\right)^{1 / 2}$, then

$$
\tan \angle C-\tan \angle B \approx \delta^{1 / 2}\left(\tilde{\xi}_{1}^{1 / 2}-\bar{\xi}_{1}^{1 / 2}\right) \approx \tan \angle D O B-\tan \angle A O B \approx 2^{\ell}\left(\frac{\delta}{\lambda}\right)^{1 / 2},
$$

which implies $\tan (\angle C-\angle B) \approx 2^{\ell}\left(\frac{\delta}{\lambda}\right)^{1 / 2}$. As a result of $\angle A P D+\angle A E D=\angle D E I+$ $\angle A E D=180^{\circ}$, then $\angle A P D=\angle D E I=\angle C-\angle B \approx 2^{\ell}\left(\frac{\delta}{\lambda}\right)^{1 / 2}$. 


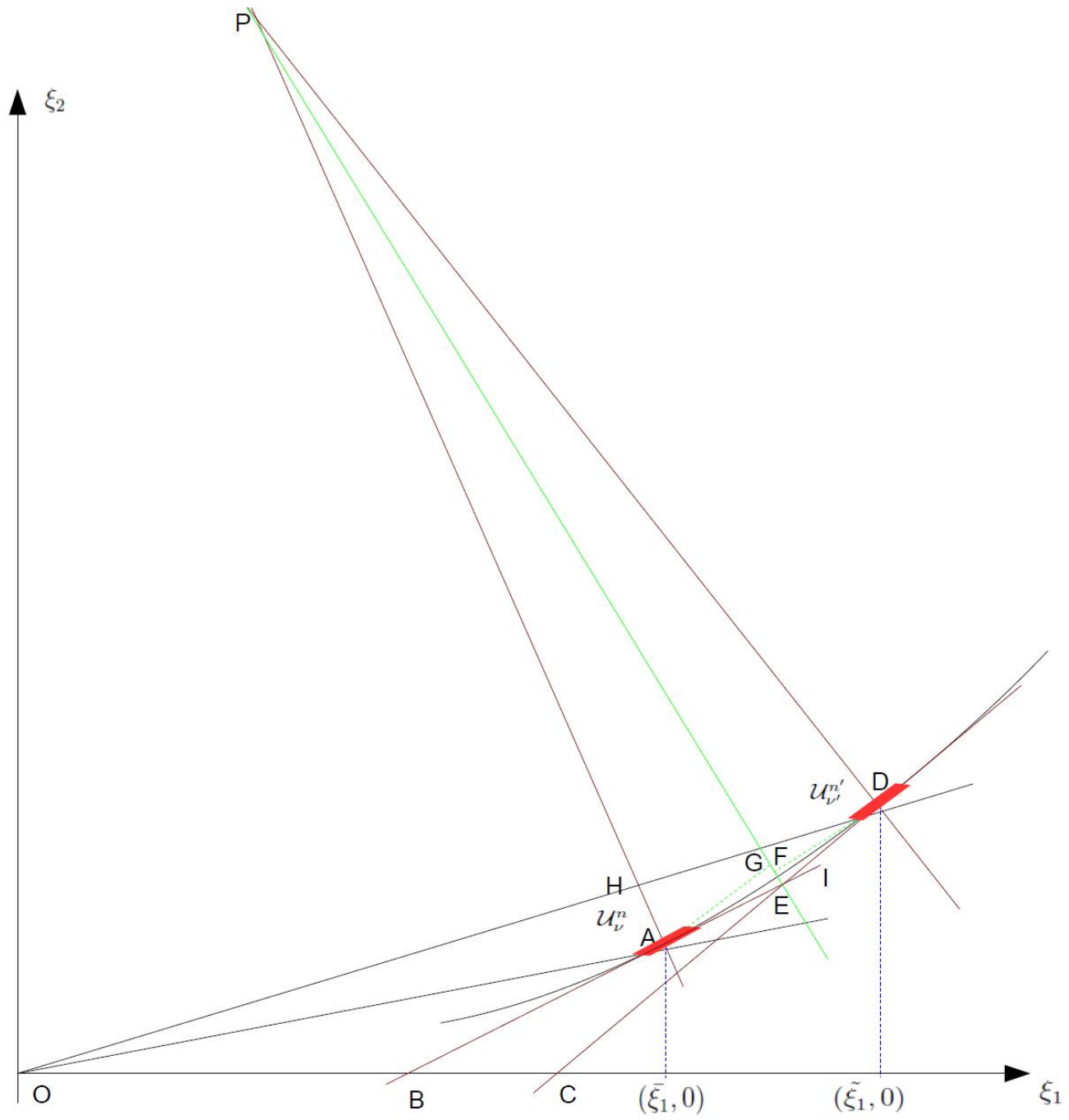

Figure 5: Curve $q_{\delta}(\xi)=1$

Since $q_{\delta}(\xi)=1$, then $\delta^{-1 / 2} \frac{\xi_{2}}{\xi_{1}}=\xi_{1}^{1 / 2}$, and the slope of the line $P A$ (the line $P D$ ) is equal to $\frac{3}{2} \delta^{1 / 2} \bar{\xi}_{1}^{1 / 2}\left(\frac{3}{2} \delta^{1 / 2} \tilde{\xi}_{1}^{1 / 2}\right)$. So $\tilde{\xi}_{1}^{1 / 2}-\bar{\xi}_{1}^{1 / 2}=\delta^{-1 / 2}(\tan \angle D O B-\tan \angle A O B) \approx$ $\delta^{-1 / 2} 2^{\ell}\left(\frac{\delta}{\lambda}\right)^{1 / 2}=2^{\ell} / \lambda^{1 / 2}$. Then the line $P A$ can be written as

$$
\xi_{2}=-\frac{\xi_{1}}{\frac{3}{2} \delta^{1 / 2} \bar{\xi}_{1}^{1 / 2}}+\delta^{1 / 2} \bar{\xi}_{1}^{3 / 2}+\frac{2}{3} \delta^{-1 / 2} \bar{\xi}_{1}^{1 / 2}
$$

and the line $P D$ is

$$
\xi_{2}=-\frac{\xi_{1}}{\frac{3}{2} \delta^{1 / 2} \tilde{\xi}_{1}^{1 / 2}}+\delta^{1 / 2} \tilde{\xi}_{1}^{3 / 2}+\frac{2}{3} \delta^{-1 / 2} \tilde{\xi}_{1}^{1 / 2} .
$$

It's easy to compute the point $P \approx\left(-\frac{1}{\delta}, \frac{1}{\delta}\right)$. The claim was proved. 
Since $A G \perp P G, D F \perp P F, P A \perp A E$ and $P D \perp D E$, then $\angle G A E=\angle A P G \approx 2^{\ell}\left(\frac{\delta}{\lambda}\right)^{1 / 2}$ and $\angle E D F=\angle D P F \approx 2^{\ell}\left(\frac{\delta}{\lambda}\right)^{1 / 2}$.

The projection on $P G$ of the width of $\mathcal{U}_{\nu}^{n}$ is equal to $\sin \angle G A E \times \frac{1}{\sqrt{\delta \lambda}} \approx \frac{2^{\ell}}{\lambda} \geq \lambda^{-1}$. For $\nu$, $\nu^{\prime}$ with $\left|\nu-\nu^{\prime}\right| \approx 2^{\ell}, \Lambda_{\nu}^{n}+\Lambda_{\nu^{\prime}}^{n^{\prime}}$ is comparable to a rectangle of size $2^{\ell} \lambda^{-1} \times \frac{1}{\sqrt{\delta \lambda}} \times 1 /\left(\delta \lambda^{1 / 2}\right)$. In other words, $\operatorname{vol}\left(\Lambda_{\nu}^{n}+\Lambda_{\nu^{\prime}}^{n^{\prime}}\right) \approx 2^{\ell} \delta^{-1 / 2} \lambda^{-2}$.

Next we define

$$
\Omega=\left\{(\eta, \tau): \sum_{\left|\nu-\nu^{\prime}\right| \approx 2^{\ell}} \chi_{\Lambda_{\nu}^{n}+\Lambda_{\nu^{\prime}}^{n^{\prime}}}(\eta, \tau) \geq \frac{1}{2} \max _{(\eta, \tau)} \sum_{\left|\nu-\nu^{\prime}\right| \approx 2^{\ell}} \chi_{\Lambda_{\nu}^{n}+\Lambda_{\nu^{\prime}}^{n^{\prime}}}(\eta, \tau)\right\} .
$$

Since the sum is equally distributed on $\Omega$, one can check that

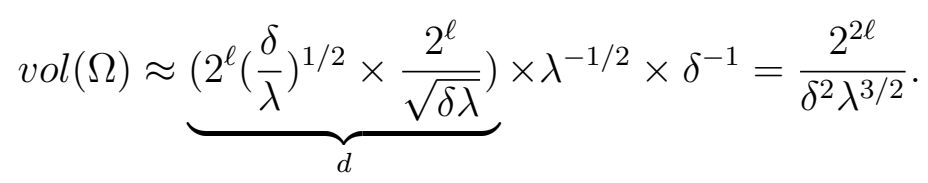

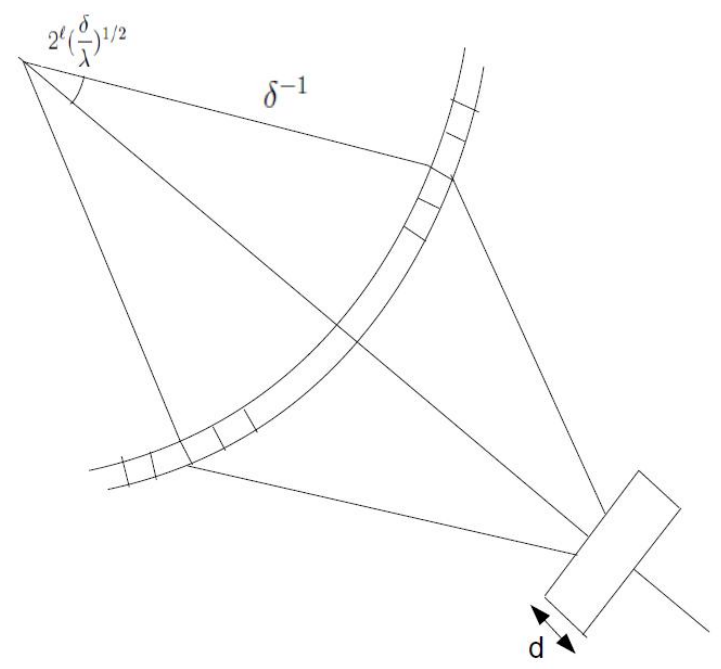

Figure $6: \Lambda_{\nu}^{n}+\Lambda_{\nu^{\prime}}^{n^{\prime}}$ for $\left|\nu-\nu^{\prime}\right| \approx 2^{\ell}$

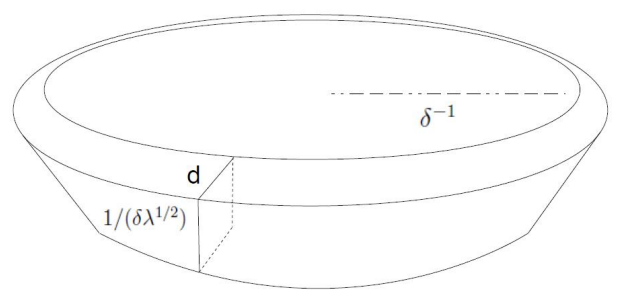

Figure $7: \Omega$ 
Set $\bar{C}=\max _{(\eta, \tau)} \sum_{\left|\nu-\nu^{\prime}\right| \approx 2^{\ell}} \chi_{\Lambda_{\nu}^{n}+\Lambda_{\nu^{\prime}}^{n^{\prime}}}(\eta, \tau)$, then

$$
\begin{aligned}
\bar{C} \operatorname{vol}(\Omega)=\int_{\Omega} \bar{C} d \eta d \tau & \leq 2 \int_{\mathbb{R}^{3}} \sum_{\left|\nu-\nu^{\prime}\right| \approx 2^{\ell}} \chi_{\Lambda_{\nu}^{n}+\Lambda_{\nu^{\prime}}^{n^{\prime}}}(\eta, \tau) d \eta d \tau \\
& =2 \sum_{\left|\nu-\nu^{\prime}\right| \approx 2^{\ell}} \operatorname{vol}\left(\Lambda_{\nu}^{n}+\Lambda_{\nu^{\prime}}^{n^{\prime}}\right) \\
& \lesssim 2^{\ell}\left(\frac{\lambda}{\delta}\right)^{1 / 2} 2^{\ell} \delta^{-3 / 2} \lambda^{-2} \lesssim 2^{2 \ell} \frac{1}{\delta^{2} \lambda^{3 / 2}}
\end{aligned}
$$

together with the estimate of $\operatorname{vol}(\Omega)$, we know $\bar{C} \leq C$, i.e. $\sum_{\left|\nu-\nu^{\prime}\right| \approx 2^{\ell}} \chi_{\Lambda_{\nu}^{n}+\Lambda_{\nu^{\prime}}^{n^{\prime}}}(\eta, \tau) \leq$ $C$.

Let us continue our estimate for $\left\|\left(\sum_{n}\left|P^{n} \sum_{\nu} Q_{\nu}\left(\mathcal{F}_{\lambda, \nu}^{\delta} f_{n}\right)\right|^{2}\right)^{1 / 2}\right\|_{L^{4}}$. By Plancherel's theorem and the above overlap lemma, we have

$$
\begin{aligned}
& \left\|\left(\sum_{n}\left|P^{n} \sum_{\nu} Q_{\nu}\left(\mathcal{F}_{\lambda, \nu}^{\delta} f_{n}\right)\right|^{2}\right)^{1 / 2}\right\|_{L^{4}}^{4} \\
& =\int_{\mathbb{R}^{3}}\left(\sum_{n}\left|P^{n} \sum_{\nu} Q_{\nu}\left(\mathcal{F}_{\lambda, \nu}^{\delta} f_{n}\right)\right|^{2}\right)^{2} d y d t \\
& =\int_{\mathbb{R}^{3}} \sum_{n}\left|P^{n} \sum_{\nu} Q_{\nu}\left(\mathcal{F}_{\lambda, \nu}^{\delta} f_{n}\right)\right|^{2} \sum_{n^{\prime}}\left|P^{n^{\prime}} \sum_{\nu^{\prime}} Q_{\nu^{\prime}}\left(\mathcal{F}_{\lambda, \nu^{\prime}}^{\delta} f_{n^{\prime}}\right)\right|^{2} d y d t \\
& =\int_{\mathbb{R}^{3}} \sum_{n, n^{\prime}}\left|\sum_{\nu, \nu^{\prime}} P^{n} Q_{\nu}\left(\mathcal{F}_{\lambda, \nu}^{\delta} f_{n}\right) P^{n^{\prime}} Q_{\nu^{\prime}}\left(\mathcal{F}_{\lambda, \nu^{\prime}}^{\delta} f_{n^{\prime}}\right)\right|^{2} d y d t \\
& =\int_{\mathbb{R}^{3}} \sum_{n, n^{\prime}}\left|\sum_{\nu, \nu^{\prime}} \chi_{U_{\nu}^{n}+U_{\nu^{\prime}}^{n^{\prime}}}(\eta, \tau)\left(P^{n} Q_{\nu}\left(\mathcal{F}_{\lambda, \nu}^{\delta} f_{n}\right)\right)^{\wedge} *\left(P^{n^{\prime}} Q_{\nu^{\prime}}\left(\mathcal{F}_{\lambda, \nu^{\prime}}^{\delta} f_{n^{\prime}}\right)\right)^{\wedge}(\eta, \tau)\right|^{2} d \eta d \tau \\
& \leq \int_{\mathbb{R}^{3}} \sum_{n, n^{\prime}} \sum_{\nu, \nu^{\prime}} \chi_{U_{\nu}^{n}+U_{\nu^{\prime}}^{n^{\prime}}(\eta, \tau)}^{2} \sum_{\nu, \nu^{\prime}}\left|\left(P^{n} Q_{\nu}\left(\mathcal{F}_{\lambda, \nu}^{\delta} f_{n}\right)\right)^{\wedge} *\left(P^{n^{\prime}} Q_{\nu^{\prime}}\left(\mathcal{F}_{\lambda, \nu^{\prime}}^{\delta} f_{n^{\prime}}\right)\right)^{\wedge}(\eta, \tau)\right|^{2} d \eta d \tau \\
& \leq C\left(\frac{\lambda}{\delta}\right)^{2 \epsilon} \log _{2}\left(\frac{\lambda}{\delta}\right)^{1 / 2} \int_{\mathbb{R}^{3}} \sum_{n, n^{\prime}} \sum_{\nu, \nu^{\prime}}\left|P^{n} Q_{\nu}\left(\mathcal{F}_{\lambda, \nu}^{\delta} f_{n}\right) P^{n^{\prime}} Q_{\nu^{\prime}}\left(\mathcal{F}_{\lambda, \nu^{\prime}}^{\delta} f_{n^{\prime}}\right)\right|^{2} d y d t \\
& =C\left(\frac{\lambda}{\delta}\right)^{2 \epsilon} \log _{2}\left(\frac{\lambda}{\delta}\right)^{1 / 2}\left\|\left(\sum_{n, \nu}\left|P^{n} Q_{\nu}\left(\mathcal{F}_{\lambda, \nu}^{\delta} f_{n}\right)\right|^{2}\right)^{1 / 2}\right\| \|_{L^{4}}^{4}
\end{aligned}
$$

Hence by Lemma 3.0.4, it suffices to estimate $\left\|\left(\sum_{n, \nu}\left|P^{n} \mathcal{F}_{\lambda, \nu}^{\delta} f_{n}\right|^{2}\right)^{1 / 2}\right\|_{L^{4}}$. 
Furthermore,

$$
\begin{aligned}
& \left\|\left(\sum_{n, \nu}\left|P^{n}\left(\mathcal{F}_{\lambda, \nu}^{\delta} f_{n}\right)\right|^{2}\right)^{1 / 2}\right\|_{L^{4}}^{4} \\
& =\left\|\left(\sum_{n, \nu}\left|\lambda^{1 / 2} e^{-i \lambda^{1 / 2} n t} \check{\varphi}\left(\lambda^{1 / 2} \cdot\right) *_{t} \mathcal{F}_{\lambda, \nu}^{\delta} f_{n}(x, t)\right|^{2}\right)^{1 / 2}\right\|_{L^{4}}^{4} \\
& \leq C_{N}^{\prime}\left\|\left(\sum_{n, \nu}\left|\lambda^{1 / 2} \int_{\mathbb{R}} \frac{\left|\mathcal{F}_{\lambda, \nu}^{\delta} f_{n}(y, t-s)\right|}{\left(1+\lambda^{1 / 2}|s|\right)^{N}} d s\right|^{2}\right)^{1 / 2}\right\|_{L^{4}}^{4} \\
& \leq C_{N}^{\prime} \int_{\mathbb{R}^{2}} \int_{\mathbb{R}}\left[\sum_{n, \nu} \lambda^{1 / 2} \int_{\mathbb{R}} \frac{1}{\left(1+\lambda^{1 / 2}|s|\right)^{N}} d s \lambda^{1 / 2} \int_{\mathbb{R}} \frac{\left|\mathcal{F}_{\lambda, \nu}^{\delta} f_{n}(y, t-s)\right|^{2}}{\left(1+\lambda^{1 / 2}|s|\right)^{N}} d s\right]^{2} d y d t \\
& \leq C_{N}^{\prime \prime} \int_{\mathbb{R}^{2}} \int_{\mathbb{R}}\left(\sum_{n, \nu} \lambda^{1 / 2} \int_{\mathbb{R}} \frac{\left|\mathcal{F}_{\lambda, \nu}^{\delta} f_{n}(y, t-s)\right|^{2}}{\left(1+\lambda^{1 / 2}|s|\right)^{N}} d s\right)^{2} d y d t \\
& \leq C_{N}^{\prime \prime}\left\|\sum_{n, \nu} \lambda^{1 / 2} \int_{\mathbb{R}} \frac{\left|\mathcal{F}_{\lambda, \nu}^{\delta} f_{n}(y, t-s)\right|^{2}}{\left(1+\lambda^{1 / 2}|s|\right)^{N}} d s\right\|_{L^{2}}^{2} \\
& \leq C_{N}^{\prime \prime}\left(\int_{\mathbb{R}} \lambda^{1 / 2}\left(1+\lambda^{1 / 2}|s|\right)^{-N}\left\|\sum_{n, \nu}\left|\mathcal{F}_{\lambda, \nu}^{\delta} f_{n}(y, t-s)\right|^{2}\right\|_{L^{2}} d s\right)^{2} \\
& \leq C_{N}^{\prime \prime \prime}\left\|\left(\sum_{n, \nu}\left|\mathcal{F}_{\lambda, \nu}^{\delta} f_{n}(y, t)\right|^{2}\right)^{1 / 2}\right\| \|_{L^{4}}^{4}
\end{aligned}
$$

where $*_{t}$ means that convolution acts only on the variable $t$.

At present we have

$$
\left\|\mathcal{F}_{\lambda}^{\delta} f\right\|_{L^{4}} \leq C \lambda^{1 / 8}\left(\frac{\lambda}{\delta}\right)^{\epsilon / 2}\left[\log _{2}\left(\frac{\lambda}{\delta}\right)^{1 / 2}\right]^{1 / 4}\left\|\left(\sum_{n, \nu}\left|\mathcal{F}_{\lambda, \nu}^{\delta} f_{n}(x, t)\right|^{2}\right)^{1 / 2}\right\|_{L^{4}}+C_{N} \lambda^{-N}\|f\|_{L^{4}}
$$

It remains to estimate $\left\|\left(\sum_{n, \nu}\left|\mathcal{F}_{\lambda, \nu}^{\delta} f_{n}(x, t)\right|^{2}\right)^{1 / 2}\right\|_{L^{4}}$. For $m=\left(m_{1}, m_{2}\right) \in \mathbb{Z}^{2}$, we define the operator $P_{m}^{\delta}$ acting on the functions in $\mathbb{R}^{2}$ by

$$
\left(P_{m}^{\delta} f\right)^{\wedge}(\xi)=\varphi\left(\frac{\delta}{\lambda^{1 / 2}} \xi_{1}-m_{1}\right) \varphi\left(\frac{\delta}{\lambda^{1 / 2}} \xi_{2}-m_{2}\right) \widehat{f}(\xi)
$$

then $f=\sum_{m \in \mathbb{Z}^{2}} P_{m}^{\delta} f$. 


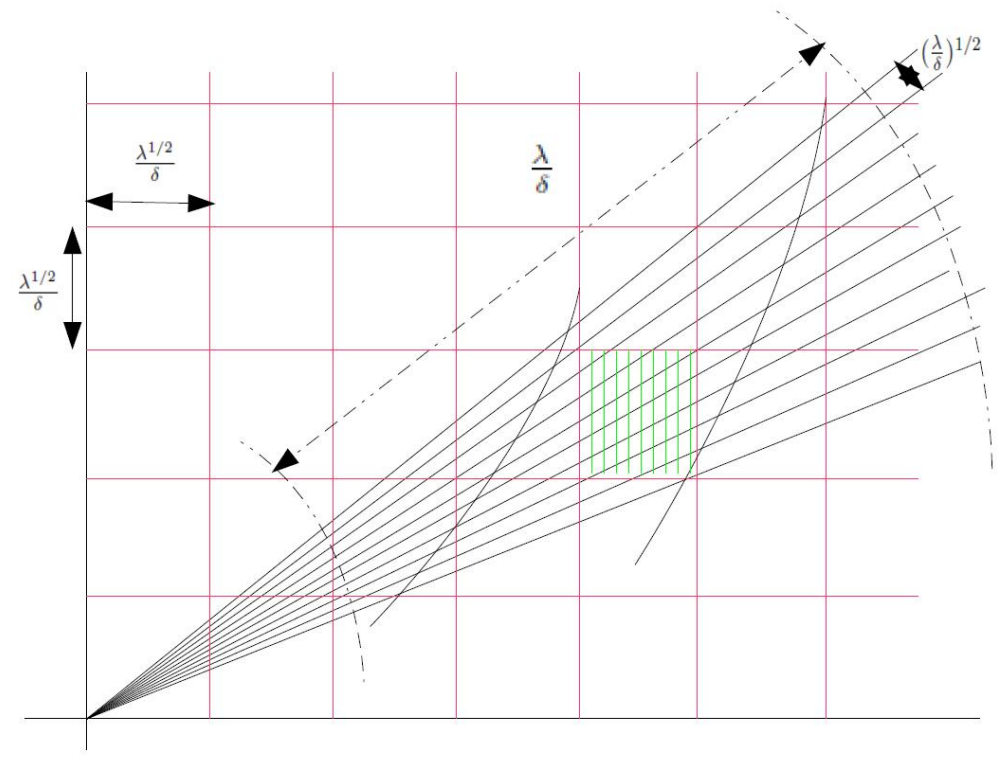

Figure 8: Card $\left\{(n, \nu): m \in J_{n, \nu}\right\} \lesssim \delta^{-1 / 2}$

The definition of $f_{n}$ and the support properties of $\chi_{\nu}$ imply

$$
\mathcal{F}_{\lambda, \nu}^{\delta} f_{n}=\mathcal{F}_{\lambda, \nu}^{\delta} f_{n, \nu}
$$

where $f_{n, \nu}=\sum_{m \in J_{n, \nu}} P_{m}^{\delta} f$ and the index set $J_{n, \nu}$ is contained in the set of all $m \in \mathbb{Z}^{2}$ such that $\chi_{\nu} \widehat{P_{m}^{\delta} f_{n}}$ is not identically zero. It follows from the above discussion that

$$
\operatorname{card}\left\{J_{n, \nu}\right\} \leq C
$$

Meanwhile, we also get

$$
\operatorname{card}\left\{(n, \nu): m \in J_{n, \nu}\right\} \lesssim \delta^{-1 / 2}
$$

Let the kernel $K_{\lambda, \nu, n}^{\delta}(y, t ; x)$ of $\mathcal{F}_{\lambda, \nu}^{\delta} f_{n, \nu}$ be

$$
\rho_{1}(y, t) \int_{\mathbb{R}^{2}} e^{i\left((y-x) \cdot \xi+E(\xi)+t q_{\delta}(\xi)\right)} \rho_{0}\left(\frac{\delta}{\lambda}|\xi|\right) \tilde{\chi}\left(\frac{\xi_{1}}{\xi_{2}}\right) a(\xi, t) \chi_{\nu}(\xi) \varphi\left(\left(\lambda^{-1 / 2} q_{\delta}(\xi)-n\right) / 10\right) d \xi
$$

We need the following estimates. Notice that (3.23) is an estimate of a Kakeya-type maximal operator.

Lemma 3.0.6. For fixed $n, \nu$ and $g \in L^{2}\left(\mathbb{R}^{2}\right)$, then

$$
\int_{\mathbb{R}^{2}}\left|K_{\lambda, \nu, n}^{\delta}(y, t ; x)\right| d x \leq C
$$




$$
\left(\int_{\mathbb{R}^{2}} \sup _{\nu, n}\left\{\int_{\mathbb{R}^{2}} \int_{\mathbb{R}}\left|K_{\lambda, \nu, n}^{\delta}(y, t ; x) \| g(y, t)\right| d y d t\right\}^{2} d x\right)^{1 / 2} \leq C \delta^{-1 / 2}\left(\log _{2} \frac{\lambda^{1 / 2}}{\delta}\right)^{2}\|g\|_{L^{2}} .
$$

Lemma 3.0.7. [26] For $2 \leq p \leq \infty$,

$$
\left\|\left(\sum_{m \in \mathbb{Z}^{2}}\left|P_{m}^{\delta} f(y)\right|^{2}\right)^{1 / 2}\right\|_{L^{p}\left(\mathbb{R}^{2}\right)} \leq C\|f\|_{L^{p}\left(\mathbb{R}^{2}\right)} .
$$

Supposing that Lemma 3.0.6 holds true, together with (3.19), (3.20) and Lemma 3.0.7, a duality argument and Hölder's inequality give

$$
\begin{aligned}
& \left\|\left(\sum_{n, \nu}\left|\mathcal{F}_{\lambda, \nu}^{\delta} f_{n}(y, t)\right|^{2}\right)^{1 / 2}\right\|_{L^{4}}^{2} \\
& =\left.\sup _{\|g\|_{L^{2}}=1}\left|\int_{\mathbb{R}^{2}} \int_{\mathbb{R}} \sum_{n, \nu}\right| \mathcal{F}_{\lambda, \nu}^{\delta} f_{n}(y, t)\right|^{2} g(y, t) d y d t \mid \\
& =\sup _{\|g\|_{L^{2}}=1} \sum_{n, \nu} \int_{\mathbb{R}^{2}} \int_{\mathbb{R}}\left|\int_{\mathbb{R}^{2}}\left(K_{\lambda, \nu, n}^{\delta}(y, t ; x)\right)^{1 / 2}\left[\left(K_{\lambda, \nu, n}^{\delta}(y, t ; x)\right)^{1 / 2} f_{n, \nu}(x)\right] d x\right|^{2}|g(y, t)| d y d t \\
& \leq \sup _{\|g\|_{L^{2}}=1} \sum_{n, \nu} \int_{\mathbb{R}^{2}} \int_{\mathbb{R}} \int_{\mathbb{R}^{2}}\left|K_{\lambda, \nu, n}^{\delta}(y, t ; x)\right| d x \int_{\mathbb{R}^{2}}\left|K_{\lambda, \nu, n}^{\delta}(y, t ; x) \| f_{n, \nu}(x)\right|^{2} d x|g(y, t)| d y d t \\
& \leq C \sup _{\|g\|_{L^{2}}=1} \sum_{n, \nu} \int_{\mathbb{R}^{2}} \int_{\mathbb{R}} \int_{\mathbb{R}^{2}}\left|K_{\lambda, \nu, n}^{\delta}(y, t ; x) \| f_{n, \nu}(x)\right|^{2} d x|g(y, t)| d y d t \\
& \leq C \sup _{\|g\|_{L^{2}}=1} \sum_{n, \nu} \int_{\mathbb{R}^{2}}\left|f_{n, \nu}(x)\right|^{2} \int_{\mathbb{R}^{2}} \int_{\mathbb{R}}\left|K_{\lambda, \nu, n}^{\delta}(y, t ; x) \| g(y, t)\right| d y d t d x \\
& \leq C^{\prime} \sup _{\|g\|_{L^{2}}=1} \int_{\mathbb{R}^{2}} \sum_{n, \nu} \sum_{m \in J_{n, \nu}}\left|P_{m}^{\delta} f(x)\right|^{2} \sup _{n, \nu} \int_{\mathbb{R}^{2}} \int_{\mathbb{R}}\left|K_{\lambda, \nu, n}^{\delta}(y, t ; x) \| g(y, t)\right| d y d t d x \\
& \leq C^{\prime} \sup _{\|g\|_{L^{2}}=1} \int_{\mathbb{R}^{2}} \sum_{m \in \mathbb{Z}^{2}} \sum_{\left\{(n, \nu): m \in J_{n, \nu}\right\}}\left|P_{m}^{\delta} f(x)\right|^{2} \sup _{n, \nu} \int_{\mathbb{R}^{2}} \int_{\mathbb{R}}\left|K_{\lambda, \nu, n}^{\delta}(y, t ; x) \| g(y, t)\right| d y d t d x \\
& \leq C^{\prime \prime} \delta^{-1 / 2} \sup _{\|g\|_{L^{2}}=1}\left\|\left(\sum_{m \in \mathbb{Z}^{2}}\left|P_{m}^{\delta} f(x)\right|^{2}\right)^{1 / 2}\right\|_{L^{4}}^{2} \\
& \quad \times\left(\int_{\mathbb{R}^{2}} \sup _{\nu, n}\left\{\int_{\mathbb{R}^{2}} \int_{\mathbb{R}}\left|K_{\lambda, \nu, n}^{\delta}(y, t ; x) \| g(y, t)\right| d y d t\right\}^{2} d x\right)^{1 / 2} \\
& \leq C^{\prime \prime \prime} \delta^{-1}\left(\log _{2} \frac{\lambda^{1 / 2}}{\delta}\right)^{2}\|f\|_{L^{4}}^{2} .
\end{aligned}
$$


Therefore,

$$
\begin{aligned}
\left\|\mathcal{F}_{\lambda}^{\delta} f\right\|_{L^{4}} & \leq C \delta^{-1 / 2} \lambda^{1 / 8}\left(\frac{\lambda}{\delta}\right)^{\epsilon / 2}\left[\log _{2}\left(\frac{\lambda}{\delta}\right)^{1 / 2}\right]^{1 / 4} \log _{2} \frac{\lambda^{1 / 2}}{\delta}\|f\|_{L^{4}}+C_{N} \lambda^{-N}\|f\|_{L^{4}} \\
& \leq C \lambda^{1 / 8+\epsilon_{1}} \delta^{-\left(1 / 2+\epsilon_{2}\right)}\|f\|_{L^{4}},
\end{aligned}
$$

where $\epsilon_{1}$ and $\epsilon_{2}$ are very small.

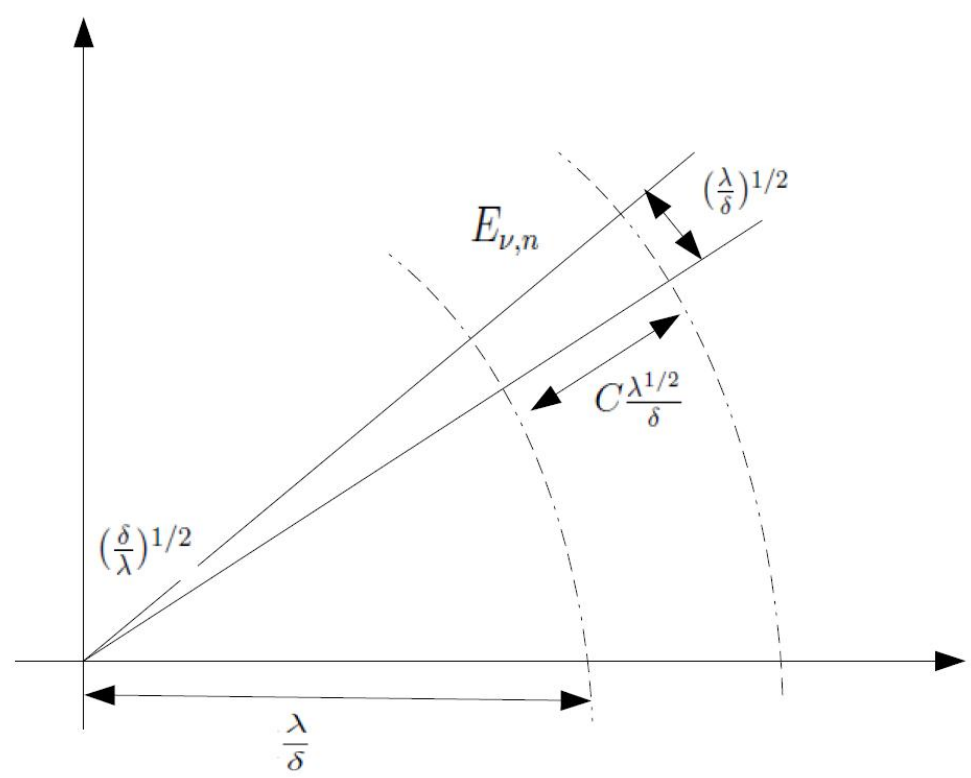

Figure $9: E_{\nu, n}$

We turn to prove Lemma 3.0.6. By (3.21), we put

$$
E_{\nu, n}:=\left\{\xi: \rho_{0}\left(\frac{\delta}{\lambda}|\xi|\right) \tilde{\chi}\left(\frac{\xi_{1}}{\xi_{2}}\right) a(\xi, t) \chi_{\nu}(\xi) \varphi\left(\left(\lambda^{-1 / 2} q_{\delta}(\xi)-n\right) / 10\right) \neq 0\right\}
$$

and consider the size of $E_{\nu, n}$ (see Figure 9), first we can adopt some ideas from the proof of inequality (2.55) to prove inequality (3.22), here we still use similar notations. After a rotation by the transformation $\tilde{T}$, we put $\tilde{\tilde{\Psi}}(y, \xi)=\tilde{T} \xi \cdot y+E(\tilde{T} \xi)+t q_{\delta}(\tilde{T} \xi)$ and $h(\xi)=\tilde{\tilde{\Psi}}(y, \xi)-\tilde{\tilde{\Psi}}_{\xi}(y, \bar{\xi}) \cdot \xi$, where $\bar{\xi}=(1,0)$. Since $\tilde{\tilde{\Psi}}$ is homogeneous of degree one, $|\xi| \approx \frac{\lambda}{\delta}$ and $\left|\xi_{2}\right| \leq C\left(\frac{\lambda}{\delta}\right)^{1 / 2}$, then we can get some similar results with (2.58), (2.59), $(2.60)$,

$$
\begin{gathered}
\left|\left(\frac{\partial}{\partial \xi_{1}}\right)^{N} h(\xi)\right| \lesssim\left(\frac{\lambda}{\delta}\right)^{-N},\left|\left(\frac{\partial}{\partial \xi_{2}}\right)^{N} h(\xi)\right| \lesssim\left(\frac{\lambda}{\delta}\right)^{-N / 2}, \\
\left|\left(\frac{\partial}{\partial \xi_{1}}\right)^{N}\left(\chi_{\nu} \circ \tilde{T}\right)(\xi)\right| \lesssim\left(\frac{\lambda}{\delta}\right)^{-N},\left|\left(\frac{\partial}{\partial \xi_{2}}\right)^{N}\left(\chi_{\nu} \circ \tilde{T}\right)(\xi)\right| \lesssim\left(\frac{\lambda}{\delta}\right)^{-N / 2}, \quad N \geq 1 .
\end{gathered}
$$


Furthermore, set $\mathcal{L}:=I-\frac{\lambda}{\delta^{2}} \frac{\partial^{2}}{\partial \xi_{1}^{2}}-\frac{\lambda}{\delta} \frac{\partial^{2}}{\partial \xi_{2}^{2}}$ and $D_{t}(z):=\left(t^{2} z_{1}, t z_{2}\right)$. Because of (2.63), (3.25), (3.26) and $\mathcal{L}^{N}\left(\varphi\left(\left(\lambda^{-1 / 2} q_{\delta}(T \xi)-n\right) / 10\right)\right) \leq C$, then

$$
\mathcal{L}^{N}\left(e^{i h(\xi)} \rho_{0}\left(\frac{\delta}{\lambda}|\xi|\right) \tilde{\chi}\left(\frac{\xi_{1}}{\xi_{2}}\right) a(\xi, t) \chi_{\nu}(\xi) \varphi\left(\left(\lambda^{-1 / 2} q_{\delta}(\xi)-n\right) / 10\right)\right) \leq C .
$$

Noting that the area of the region $E_{\nu, n}$ is at most $C \frac{\lambda}{\delta^{3 / 2}}$, so by integration by parts in $\xi$, we obtain

$$
\begin{aligned}
\left|K_{\lambda, \nu, n}^{\delta}(y, t ; x)\right| & \leq \frac{C_{N}^{\prime} \lambda / \delta^{3 / 2}}{\left(1+\frac{\lambda}{\delta^{2}}\left|\left(\tilde{\Psi}_{\xi}(y-x, \bar{\xi})\right)_{1}\right|^{2}+\frac{\lambda}{\delta}\left|\left(\tilde{\tilde{\Psi}}_{\xi}(y-x, \bar{\xi})\right)_{2}\right|^{2}\right)^{N}} \\
& \leq \frac{C_{N} \lambda / \delta^{3 / 2}}{\left(1+\left|D_{\delta^{-1 / 2}} \lambda^{1 / 2}\left(\tilde{\tilde{\Psi}}_{\xi}(y-x, \bar{\xi})\right)\right|\right)^{2 N}},
\end{aligned}
$$

where

$$
\left|\left(\tilde{\tilde{\Psi}}_{\xi}(y-x, \bar{\xi})\right)_{1}\right|=\left|\left\langle y-x+\nabla E\left(\xi_{\nu}\right)+t \nabla q_{\delta}\left(\xi_{\nu}\right), \xi_{\nu}\right\rangle\right|
$$

and

$$
\left|\left(\tilde{\tilde{\Psi}}_{\xi}(y-x, \bar{\xi})\right)_{2}\right|=\left|y-x+\nabla E\left(\xi_{\nu}\right)+t \nabla q_{\delta}\left(\xi_{\nu}\right)-\left\langle y-x+\nabla E\left(\xi_{\nu}\right)+t \nabla q_{\delta}\left(\xi_{\nu}\right), \xi_{\nu}\right\rangle \xi_{\nu}\right| .
$$

Inequality (3.27) implies inequality (3.22).

We introduce some notations. Given a direction $\xi_{\nu}=\left(\cos \theta_{\nu}, \sin \theta_{\nu}\right) \in S^{1}$, let $\gamma_{\theta_{\nu}} \subset \mathbb{R}^{3}$ be the ray defined by

$$
\gamma_{\theta_{\nu}}=\left\{(y, t): y+\nabla E\left(\cos \theta_{\nu}, \sin \theta_{\nu}\right)+t \nabla q_{\delta}\left(\cos \theta_{\nu}, \sin \theta_{\nu}\right)=0\right\} .
$$

From inequality (3.27), we note that for fixed $x$, the kernels $K_{\lambda, \nu, n}^{\delta}(y, t ; x)$ are essentially supported in a rectangle of size $\frac{\delta}{\lambda^{1 / 2}} \times\left(\frac{\delta}{\lambda}\right)^{1 / 2} \times 1$ around $\gamma_{\theta_{\nu}}+(x, 0)$, see Figure 10 . This sheds some light on the proof of inequality (3.23).

We may assume $g \geq 0$ and choose non-negative functions $\varrho \in C_{0}^{\infty}\left(\left[\pi / 2-c_{2}, \pi / 2-c_{1}\right]\right)$ and $\beta_{0} \in C_{0}^{\infty}([0,2])$ which satisfies $\beta_{0}(r)+\sum_{\ell=1}^{\infty} \beta\left(2^{-\ell} r\right)=1$, for $r>0$. Let $|\cdot|_{D}$ be a homogeneous norm under $D_{t}(z)=\left(t^{2} z_{1}, t z_{2}\right)$, i.e. $\left|D_{t}(z)\right|_{D}=t|z|_{D}$. Let $h_{0}(\xi)=$ $\beta_{0}\left(|\xi|_{D}\right) \in C_{0}^{\infty}\left(\mathbb{R}^{2}\right)$ and $h(\xi)=\beta\left(|\xi|_{D}\right) \in C_{0}^{\infty}\left(\mathbb{R}^{2}\right)$, then $h_{0}(\xi)+\sum_{\ell=1}^{\infty} h\left(D_{2^{-\ell}} \xi\right)=1$. It is clear that for $\ell>1, \xi \neq 0, h\left(D_{2^{-\ell}} \xi\right) \neq 0 \Rightarrow 2^{\ell-1} \leq|\xi|_{D} \leq 2^{\ell+1} \Rightarrow|\xi| \gtrsim 2^{\ell}$.

Since the right side of inequality (3.27) does not depend on $n$, then the left side of 
inequality (3.23) can be controlled by

$$
\begin{aligned}
& \left(\int_{\mathbb{R}^{2}} \sup _{\nu, n}\left\{\int_{\mathbb{R}^{2}} \int_{\mathbb{R}}\left|K_{\lambda, \nu, n}^{\delta}(y, t ; x) \| g(y, t)\right| d y d t\right\}^{2} d x\right)^{1 / 2} \\
& \leq C_{N}^{\prime}\left(\left\|\sup _{\nu} \frac{1}{\delta^{3 / 2} / \lambda} \int_{\mathbb{R}^{2} \times[1 / 2,4]} h_{0}\left(D_{\delta^{-1 / 2}} \lambda^{1 / 2} \tilde{\tilde{\Psi}}_{\xi}(y, \bar{\xi})\right) g(x+y, t) d y d t\right\|_{L^{2}}\right. \\
& \left.+\left\|\sup _{\nu} \sum_{\ell=1}^{\infty} \int_{\mathbb{R}^{2} \times[1 / 2,4]} h\left(D_{2^{-\ell}}\left(D_{\delta^{-1 / 2}} \lambda^{1 / 2} \tilde{\tilde{\Psi}}_{\xi}(y, \bar{\xi})\right)\right) \frac{\lambda / \delta^{3 / 2} g(x+y, t)}{\left(1+\left|D_{\delta^{-1 / 2}} \lambda^{1 / 2} \tilde{\tilde{\Psi}}_{\xi}(y, \bar{\xi})\right|\right)^{2 N}} d y d t\right\|_{L^{2}}\right) \\
& \leq C_{N}^{\prime \prime} \sum_{\ell=0}^{\infty} 2^{-2(N-3 / 2) \ell} \|_{\nu} \sup _{\nu} \frac{1}{2^{3(\ell+1)} \delta^{3 / 2} / \lambda} \int_{\left\{(y, t) \in \mathbb{R}^{2} \times[0,1]:\left|\lambda^{1 / 2}\left(y+\nabla E\left(\xi_{\nu}\right)+t \nabla q_{\delta}\left(\xi_{\nu}\right)\right)\right|_{D} \leq 2^{\ell+1} \delta^{1 / 2}\right\}} \\
& \leq C_{N}^{\prime \prime} \sum_{\ell=0}^{\infty} 2^{-2(N-3 / 2) \ell}\left\|_{\nu} \sup _{\nu} \frac{1}{\left|R_{\nu}^{\ell}\right|} \int_{R_{\nu}^{\ell}} g(x+y, t) d y d t\right\|_{L^{2}},
\end{aligned}
$$

where

$$
R_{\nu}^{\ell}=\left\{(y, t) \in \mathbb{R}^{2} \times[1 / 2,4]:\left|\lambda^{1 / 2}\left(y+\nabla E\left(\xi_{\nu}\right)+t \nabla q_{\delta}\left(\xi_{\nu}\right)\right)\right|_{D} \leq 2^{\ell+1} \delta^{1 / 2}\right\} .
$$

From the above argument, in order to prove inequality (3.23), we need to prove an $L^{2}\left(\mathbb{R}^{3}\right) \rightarrow L^{2}\left(\mathbb{R}^{2}\right)$ maximal estimate involving averages over cuboids $R_{\nu}^{0}$ of dimensions $\frac{\delta}{\lambda^{1 / 2}} \times\left(\frac{\delta}{\lambda}\right)^{1 / 2} \times 1$, which is basically tangential to the cone $\left\{(y, t) \in \gamma_{\theta}\right.$ : $\gamma_{\theta}$ is defined as (3.28) and $\left.\theta \in \operatorname{supp} \rho\right\}$ (see Figure 10), because in a similar way one can obtain $L^{2}\left(\mathbb{R}^{3}\right) \rightarrow L^{2}\left(\mathbb{R}^{2}\right)$ maximal estimates involving averages over $\left\{R_{\nu}^{\ell}\right\}_{\nu}$ which are $\frac{2^{2 \ell} \delta}{\lambda^{1 / 2}} \times 2^{\ell}\left(\frac{\delta}{\lambda}\right)^{1 / 2} \times 1$ cuboids for $\ell=1,2, \cdots$. However, we can split every $R_{\nu}^{0}$ along its longer side of size $\left(\frac{\delta}{\lambda}\right)^{1 / 2}$ into $\delta^{-1 / 2}$ pieces, i.e. $\left\{R_{\nu, i}^{0}\right\}_{i=1}^{\delta^{-1 / 2}}$, where $R_{\nu, i}^{0}$ is a $\frac{\delta}{\lambda^{1 / 2}} \times \frac{\delta}{\lambda^{1 / 2}} \times 1$ tube around $\gamma_{\theta_{\nu, i}}+(x, 0)$ (see Figure 10). Then next we will prove a stronger maximal estimate involving averages over tubes $R_{\theta}$ defined by

$$
R_{\theta}:=\left\{(y, t) \in \mathbb{R}^{2} \times[0,1]: \operatorname{dist}\left\{(y, t), \gamma_{\theta}\right\}<\frac{\delta}{\lambda^{1 / 2}}\right\}
$$

That is because

$$
\begin{aligned}
\left\|\sup _{\nu} \frac{1}{\left|R_{\nu}^{0}\right|} \int_{R_{\nu}^{0}} g(x+y, t) d y d t\right\|_{L^{2}} & \leq \delta^{1 / 2} \sum_{i=1}^{\delta^{-1 / 2}}\left\|\sup _{\nu} \frac{1}{\left|R_{\nu, i}^{0}\right|} \int_{R_{\nu, i}^{0}} g(x+y, t) d y d t\right\|_{L^{2}} \\
& \leq \delta^{1 / 2} \sum_{i=1}^{\delta^{-1 / 2}}\left\|\sup _{\theta} \frac{1}{\left|R_{\theta}\right|} \int_{R_{\theta}} \varrho(\theta) g(x+y, t) d y d t\right\|_{L^{2}} .
\end{aligned}
$$

Hence, we would be done if we can obtain the following Kakeya type estimate. 


\section{Lemma 3.0.8.}

$$
\left\|\sup _{\theta} \frac{1}{\left|R_{\theta}\right|} \int_{R_{\theta}} \varrho(\theta) g(x-y, t) d y d t\right\|_{L^{2}} \leq C \delta^{-1 / 2}\left(\log _{2} \frac{\lambda^{1 / 2}}{\delta}\right)^{2}\|g\|_{L^{2}} .
$$

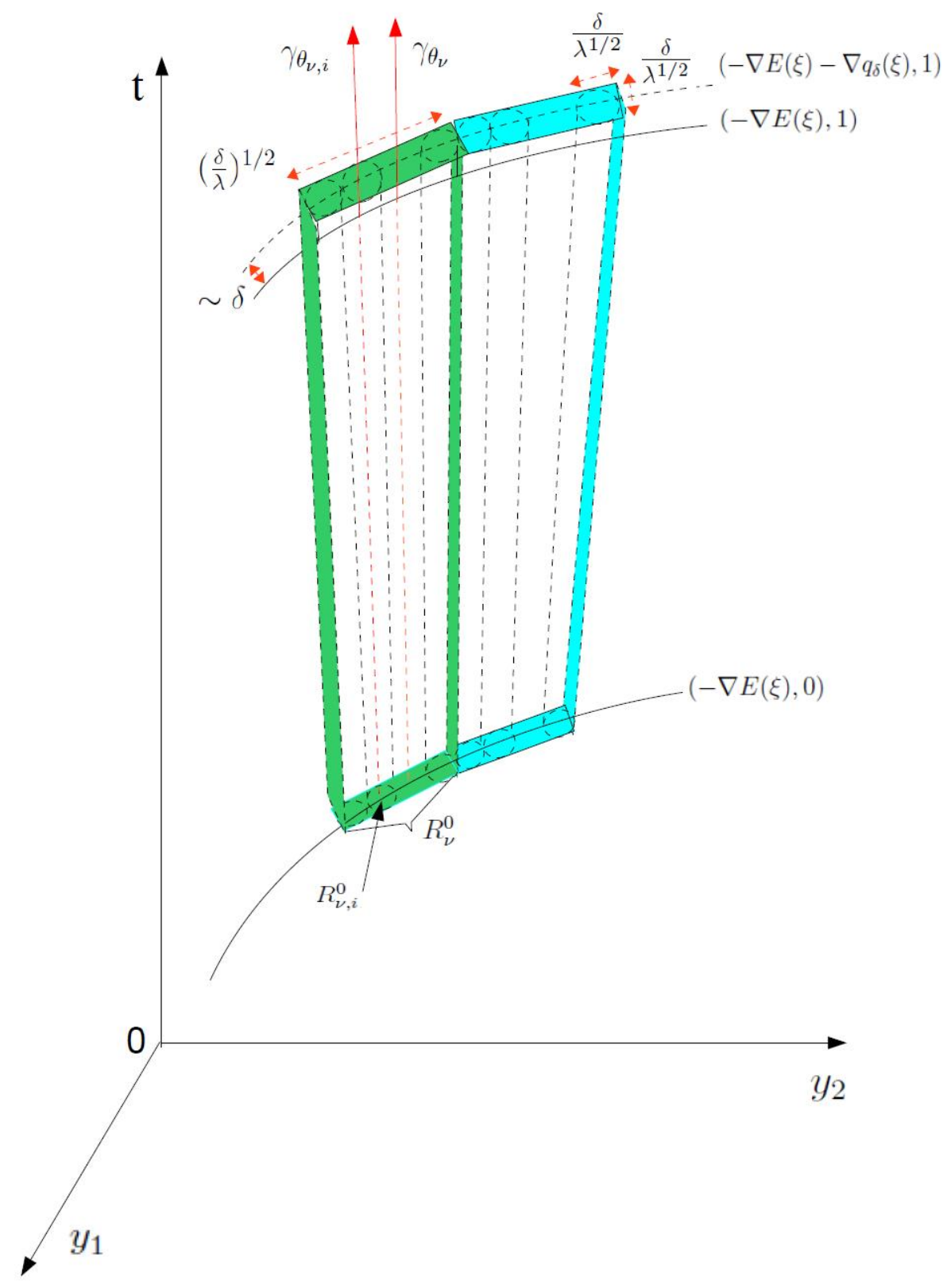

Figure 10 : cuboid $R_{\nu}^{0}$ and tube $R_{\nu, i}^{0}$

Remark 3.0.1. The constant $\delta^{-1 / 2}$ in inequality (3.30) is sharp if we take $E(\xi)=|\xi|$ 
and $q_{\delta}(\xi)=\delta|\xi|$. Let

$$
M_{\delta, \lambda} g(x)=\sup _{\theta} \frac{1}{\left|R_{\theta}\right|} \int_{R_{\theta}} \varrho(\theta) g(x-y, t) d y d t .
$$

In particular, we take $g(z, t)=\chi_{P_{\delta}}(z, t)$, where $P_{\delta}$ is a tubular neighborhood of width $\delta$ around the $t$-axis with height 1 . Assume that $x \in A_{\delta}:=\left\{x \in \mathbb{R}^{2}: 1 \leq|x| \leq\right.$ $\left.1+\delta, \quad c_{1} \leq\left|x_{1} / x_{2}\right| \leq c_{2}\right\}$. Now taking $\theta$ so that $(\cos \theta, \sin \theta)=\frac{x}{|x|}$, then we note that

$$
g * \chi_{R_{\theta}}(x)=\left|R_{\theta} \cap\left(P_{\delta}-(x, 0)\right)\right| \approx\left|R_{\theta}\right|,
$$

which implies $M_{\delta, \lambda} g(x) \gtrsim 1$ on $A_{\delta}$. Let $C_{\delta}:=\left\|M_{\delta, \lambda}\right\|_{L^{2} \rightarrow L^{2}}$. Since $\left|A_{\delta}\right| \approx \delta$, then

$$
\delta^{1 / 2} \lesssim\left\|M_{\delta, \lambda} g\right\|_{L^{2}} \leq C_{\delta} \delta \Rightarrow C_{\delta} \gtrsim \delta^{-1 / 2} .
$$

In fact, if we take $E(\xi)=\frac{\xi_{1}^{2}}{\xi_{2}}$ and $q_{\delta}(\xi)=\delta \frac{\xi_{1}^{3}}{\xi_{2}^{2}}$, in the similar way as above, we still get the same conclusion, regarding complicated computations, we omit here.

Proof. We choose a suitable $\sigma \in C_{0}^{\infty}\left(\mathbb{R}^{2}\right)$ satisfying $\check{\sigma} \geq 0$. Let $\bar{\delta}=\frac{\delta}{\lambda^{1 / 2}}$ and $\sigma_{\bar{\delta}}(\theta, t, \xi)=$ $\varrho(\theta) \sigma(\bar{\delta} \xi) \chi_{[0,1]}(t)$. Then We have

$$
\begin{aligned}
& \int_{\left\{(y, t) \in \mathbb{R}^{2} \times[0,1]: d i s t\left\{(y, t), \gamma_{\theta}\right\}<\bar{\delta}\right\}} g(x-y, t) d y d t \\
& \leq \int_{\mathbb{R}^{2}} \int_{\mathbb{R}} \varrho(\theta) \check{\sigma}\left(\frac{y+t \nabla q_{\delta}(\cos \theta, \sin \theta)+\nabla E(\cos \theta, \sin \theta)}{\bar{\delta}}\right) g(x-y, t) \chi_{[0,1]}(t) d y d t \\
& =\bar{\delta}^{2} \int_{\mathbb{R}^{2}} \int_{\mathbb{R}} \varrho(\theta)[\sigma(\bar{\delta} \cdot)]^{\vee}\left(y+t \nabla q_{\delta}(\cos \theta, \sin \theta)+\nabla E(\cos \theta, \sin \theta)\right) g(x-y, t) \\
& \quad \times \chi_{[0,1]}(t) d y d t \\
& =\bar{\delta}^{2} \int_{\mathbb{R}^{2}} \int_{\mathbb{R}} \int_{\mathbb{R}^{2}} e^{i \xi \cdot\left[x-y+t \nabla q_{\delta}(\cos \theta, \sin \theta)+\nabla E(\cos \theta, \sin \theta)\right]} \sigma_{\bar{\delta}}(\theta, t, \xi) d \xi g(y, t) d y d t \\
& =\bar{\delta}^{2} \int_{\mathbb{R}^{2}} \int_{\mathbb{R}} e^{i \xi \cdot\left[x+t \nabla q_{\delta}(\cos \theta, \sin \theta)+\nabla E(\cos \theta, \sin \theta)\right]} \sigma_{\bar{\delta}}(\theta, t, \xi) g(\hat{\xi}, t) d \xi d t \\
& =: \bar{\delta}^{2} A_{\theta} g(x),
\end{aligned}
$$

where $g(\hat{\xi}, t)$ denotes the partial Fourier transform of $g$ with respect to the $\xi$-variables. Therefore, we may further reduce to prove that

$$
\left\|\sup _{\theta}\left|A_{\theta} g\right|\right\|_{L^{2}} \leq C \delta^{-1 / 2}\left|\log _{2} \bar{\delta}\right|^{2}\|g\|_{L^{2}}
$$

In order to prove the above inequality, we need to break up the operator $A_{\theta}$. Just as before, we take $\beta \in C_{0}^{\infty}(\mathbb{R})$ and define the dyadic operators $A_{\theta}^{\tau}$ by

$$
A_{\theta}^{\tau} g(x)=\int_{\mathbb{R}^{2}} \int_{\mathbb{R}} e^{i \xi \cdot\left[x+t \nabla q_{\delta}(\cos \theta, \sin \theta)+\nabla E(\cos \theta, \sin \theta)\right]} \sigma_{\bar{\delta}}(\theta, t, \xi) \beta\left(\frac{|\xi|}{\tau}\right) g(\hat{\xi}, t) d \xi d t,
$$


so it suffices to prove that

$$
\left\|\sup _{\theta}\left|A_{\theta}^{\tau} g\right|\right\|_{L^{2}} \leq C \delta^{-1 / 2} \log _{2} \tau^{1 / 2}\|g\|_{L^{2}}, \quad \tau>2 .
$$

This is because $A_{\theta}=\sum_{1<\kappa<\left|\log _{2} \bar{\delta}\right|+C} A_{\theta}^{2^{\kappa}}+\tilde{R}_{\theta}$, where $C$ is a fixed constant and the kernel of $\tilde{R}_{\theta}$ defined by

$$
\int_{\mathbb{R}^{2}} e^{i \xi \cdot\left[(x-y)+t \nabla q_{\delta}(\cos \theta, \sin \theta)+\nabla E(\cos \theta, \sin \theta)\right]} \sigma_{\bar{\delta}}(\theta, t, \xi) \beta_{0}(|\xi|) d \xi
$$

is controlled by $\mathcal{O}\left((1+|y-x|)^{-N}\right)$ for any $N$ with bounds independent of $\theta$.

Let $\theta_{\xi}=\arg \xi$. We need to make one final reduction based on the following observation:

$$
\begin{aligned}
& \frac{\partial}{\partial \theta}\left\langle\xi, \nabla q_{\delta}(\cos \theta, \sin \theta)\right\rangle \\
& =\left\langle\xi,(-\sin \theta, \cos \theta)\left(\begin{array}{ll}
\partial_{11} q_{\delta}(\cos \theta, \sin \theta) & \partial_{12} q_{\delta}(\cos \theta, \sin \theta) \\
\partial_{21} q_{\delta}(\cos \theta, \sin \theta) & \partial_{22} q_{\delta}(\cos \theta, \sin \theta)
\end{array}\right)\right\rangle \\
& =6 \delta|\xi| \frac{\cos \theta}{\sin ^{4} \theta}\left\langle\frac{\xi}{|\xi|},(-\sin \theta, \cos \theta)\right\rangle=6 \delta|\xi| \frac{\cos \theta}{\sin ^{4} \theta} \sin \left(\theta_{\xi}-\theta\right)=0 \Leftrightarrow \theta=\theta_{\xi} .
\end{aligned}
$$

For $\ell>0$, put

$$
A_{\theta}^{\tau, \ell} g(x)=\int_{\mathbb{R}^{2}} \int_{\mathbb{R}} e^{i \xi \cdot\left[x+t \nabla q_{\delta}(\cos \theta, \sin \theta)+\nabla E(\cos \theta, \sin \theta)\right]} \sigma_{\bar{\delta}}(\theta, t, \xi) \beta\left(\frac{|\xi|}{\tau}\right) \beta_{\ell, \tau}(\xi, \theta) g(\hat{\xi}, t) d \xi d t,
$$

where $\beta_{\ell, \tau}(\xi, \theta)=\beta\left(2^{-\ell} \tau^{1 / 2}\left|\left\langle(-\sin \theta, \cos \theta), \frac{\xi}{|\xi|}\right\rangle\right|\right)$. In fact, $\left|\left\langle(-\sin \theta, \cos \theta), \frac{\xi}{|\xi|}\right\rangle\right| \leq$ $C, C$ is very small, which implies that $\ell \leq C \log _{2} \tau^{1 / 2}$. We define $A_{\theta}^{\tau, 0}=A_{\theta}^{\tau}-$ $\sum_{0<\ell \leq C \log _{2} \tau^{1 / 2}} A_{\theta}^{\tau, \ell}$.

Again we use Lemma 2.2.3, for $\ell \geq 0$,

$$
\left\|\sup _{\theta}\left|A_{\theta}^{\tau, \ell} g\right|\right\|_{L^{2}}^{2} \leq C\left(\iint\left|A_{\theta}^{\tau, \ell} g(x)\right|^{2} d \theta d x\right)^{1 / 2}\left(\iint\left|\frac{\partial}{\partial \theta} A_{\theta}^{\tau, \ell} g(x)\right|^{2} d \theta d x\right)^{1 / 2} .
$$

Inequality (3.32) will be obtained if we can show that

$$
\begin{aligned}
& \left(\iint\left|A_{\theta}^{\tau, \ell} g(x)\right|^{2} d \theta d x\right)^{1 / 2} \leq C \delta^{-1 / 2} 2^{-\ell / 2} \tau^{-1 / 4}\|g\|_{L^{2}}, \\
& \left(\iint\left|\frac{\partial}{\partial \theta} A_{\theta}^{\tau, \ell} g(x)\right|^{2} d \theta d x\right)^{1 / 2} \leq C \delta^{-1 / 2} 2^{\ell / 2} \tau^{1 / 4}\|g\|_{L^{2}}
\end{aligned}
$$

From $\beta\left(2^{-\ell} \tau^{1 / 2}\left|\left\langle(-\sin \theta, \cos \theta), \frac{\xi}{|\xi|}\right\rangle\right|\right) \neq 0$ and $\beta\left(\frac{|\xi|}{\tau}\right) \neq 0$, we know that on the support of the symbol of the operator $A_{\theta}^{\tau, \ell},\left|\left\langle(-\sin \theta, \cos \theta), \frac{\xi}{|\xi|}\right\rangle\right| \approx 2^{\ell} \tau^{-1 / 2}$ and $|\xi| \approx \tau$. 
Since

$$
\begin{aligned}
\frac{\partial}{\partial \theta} \xi \cdot \nabla E(\cos \theta, \sin \theta) & =\langle\xi, 2(-\sin \theta, \cos \theta)\rangle\left(\begin{array}{cc}
\partial_{11} E(\cos \theta, \sin \theta) & \partial_{12} E(\cos \theta, \sin \theta) \\
\partial_{21} E(\cos \theta, \sin \theta) & \partial_{22} E(\cos \theta, \sin \theta)
\end{array}\right) \\
& =\langle\xi, 2(-\sin \theta, \cos \theta)\rangle\left(\begin{array}{cc}
\frac{1}{\sin \theta} & -\frac{\cos \theta}{\sin ^{2} \theta} \\
-\frac{\cos \theta}{\sin ^{2} \theta} & \frac{\cos ^{2} \theta}{\sin ^{3} \theta}
\end{array}\right) \\
& =\frac{2}{\sin \theta}\langle\xi,(-\sin \theta, \cos \theta)\rangle\left(\begin{array}{cc}
1 & -\frac{\cos \theta}{\sin \theta} \\
-\frac{\cos \theta}{\sin \theta} & \frac{\cos ^{2} \theta}{\sin ^{2} \theta}
\end{array}\right) \\
& =|\xi| \frac{2}{\sin ^{3} \theta}\left\langle\frac{\xi}{|\xi|},(-\sin \theta, \cos \theta)\right\rangle,
\end{aligned}
$$

then

$$
\left|\frac{\partial}{\partial \theta} \xi \cdot\left[t \nabla q_{\delta}(\cos \theta, \sin \theta)+\nabla E(\cos \theta, \sin \theta)\right]\right| \leq C 2^{\ell} \tau^{1 / 2} .
$$

It is easy to check that

$$
\left|\frac{\partial}{\partial \theta} \beta\left(2^{-\ell} \tau^{1 / 2}\left|\left\langle(-\sin \theta, \cos \theta), \frac{\xi}{|\xi|}\right\rangle\right|\right)\right| \leq C 2^{-\ell} \tau^{1 / 2}
$$

one can see that $\frac{\partial}{\partial \theta} A_{\theta}^{\tau, \ell}$ behaves like $2^{\ell} \tau^{1 / 2} A_{\theta}^{\tau, \ell}$, then we only prove inequality (3.34). Employing Plancherel's theorem, We have that

$$
\begin{aligned}
& \left.\int|| A_{\theta}^{\tau, \ell} g(x)\right|^{2} d \theta d x \\
= & \int_{\mathbb{R}^{2}} \int_{\mathbb{R}} A_{\theta}^{\tau, \ell} g(x) \overline{A_{\theta}^{\tau, \ell} g(x)} d \theta d x \\
= & \int_{\mathbb{R}^{2}} \int_{\mathbb{R}}\left(\int_{\mathbb{R}^{2}} \int_{\mathbb{R}} e^{i \xi \cdot\left[x+t \nabla q_{\delta}(\cos \theta, \sin \theta)+\nabla E(\cos \theta, \sin \theta)\right]} \sigma_{\bar{\delta}}(\theta, t, \xi) \beta\left(\frac{|\xi|}{\tau}\right) \beta_{\ell, \tau}(\xi, \theta) g(\hat{\xi}, t) d \xi d t\right) \\
& \left.\times \int_{\mathbb{R}^{2}} \int_{\mathbb{R}} e^{-i \xi^{\prime} \cdot\left[x+t^{\prime} \nabla q_{\delta}(\cos \theta, \sin \theta)+\nabla E(\cos \theta, \sin \theta)\right]} \overline{\sigma_{\bar{\delta}}\left(\theta, t^{\prime}, \xi^{\prime}\right) \beta\left(\frac{\left|\xi^{\prime}\right|}{\tau}\right) \beta_{\ell, \tau}\left(\xi^{\prime}, \theta\right) g\left(\hat{\xi}^{\prime}, t^{\prime}\right)} d \xi^{\prime} d t^{\prime}\right) \\
= & \int_{\mathbb{R}^{2}} \int_{\mathbb{R}} \int_{\mathbb{R}^{2}} \int_{\mathbb{R}}\left(\int_{\mathbb{R}} e^{i\left[\left(t \xi-t^{\prime} \xi^{\prime}\right) \cdot \nabla q_{\delta}(\cos \theta, \sin \theta)+\left(\xi-\xi^{\prime}\right) \cdot \nabla E(\cos \theta, \sin \theta)\right]} \sigma_{\bar{\delta}}(\theta, t, \xi) \overline{\sigma_{\bar{\delta}}\left(\theta, t^{\prime}, \xi^{\prime}\right)}\right. \\
& \left.\times \beta_{\ell, \tau}(\xi, \theta) \overline{\beta_{\ell, \tau}\left(\xi^{\prime}, \theta\right)} d \theta\right) \delta_{0}\left(\xi^{\prime}-\xi\right) \beta\left(\frac{|\xi|}{\tau}\right) \beta\left(\frac{\xi^{\prime} \mid}{\tau}\right) g(\hat{\xi}, t) \overline{g\left(\hat{\xi}^{\prime}, t^{\prime}\right)} d \xi d t d \xi^{\prime} d t^{\prime} \\
= & \int_{\mathbb{R}^{2}} \int_{0}^{1} \int_{0}^{1}\left(\int_{\mathbb{R}} e^{i\left[\left(t-t^{\prime}\right) \xi \cdot \nabla q_{\delta}(\cos \theta, \sin \theta)\right]}\left|\rho(\theta) \beta_{\ell, \tau}(\xi, \theta)\right|^{2} d \theta\right)\left|\sigma(\bar{\delta} \xi) \beta\left(\frac{|\xi|}{\tau}\right)\right|^{2} g(\hat{\xi}, t) \\
& \times \overline{g\left(\hat{\xi}, t^{\prime}\right)} d \xi d t d t^{\prime},
\end{aligned}
$$

where $\delta_{0}$ denotes the two-dimensional Dirac delta function. 
Hence

$$
\iint\left|A_{\theta}^{\tau, \ell} g(x)\right|^{2} d \theta d x=\int_{\mathbb{R}^{2}}\left\{\int_{0}^{1} \int_{0}^{1} H^{\ell, \tau}\left(t, t^{\prime}, \xi\right)\left|\beta\left(\frac{|\xi|}{\tau}\right) \sigma(\bar{\delta} \xi)\right|^{2} g(\hat{\xi}, t) \overline{g\left(\hat{\xi}, t^{\prime}\right)} d t d t^{\prime}\right\} d \xi
$$

where

$$
H^{\ell, \tau}\left(t, t^{\prime}, \xi\right)=\int_{\mathbb{R}} e^{i\left(t-t^{\prime}\right)\left\langle\xi, \nabla q_{\delta}(\cos \theta, \sin \theta)\right\rangle}\left|\varrho(\theta) \beta_{\ell, \tau}(\xi, \theta)\right|^{2} d \theta
$$

First we claim that for $\ell>0$,

$$
\left|H^{\ell, \tau}\left(t, t^{\prime}, \xi\right)\right| \leq C 2^{\ell} \tau^{-1 / 2}\left(1+\delta 2^{2 \ell}\left|t-t^{\prime}\right|\right)^{-N},|\xi| \approx \tau
$$

Since $\left\langle(-\sin \theta, \cos \theta), \frac{\xi}{|\xi|}\right\rangle=\sin \left(\theta_{\xi}-\theta\right)$ and $\left|\theta_{\xi}-\theta\right| \approx 2^{\ell} \tau^{-1 / 2}$ on $\operatorname{supp} \beta_{\ell, \tau}$, we make the change of variable $\theta=\theta_{\xi}+\arcsin \left(2^{\ell} \tau^{-1 / 2} \omega\right)$, then

$$
\begin{aligned}
H^{\ell, \tau}\left(t, t^{\prime}, \xi\right)= & \int_{\mathbb{R}} e^{i\left(t-t^{\prime}\right)\left\langle\xi, \nabla q_{\delta}\left(\cos \left(\theta_{\xi}+\arcsin \left(2^{\ell} \tau^{-1 / 2} \omega\right)\right), \sin \left(\theta_{\xi}+\arcsin \left(2^{\ell} \tau^{-1 / 2} \omega\right)\right)\right\rangle\right.} \\
& \times\left|\varrho\left(\theta_{\xi}+\arcsin \left(2^{\ell} \tau^{-1 / 2} \omega\right)\right) \beta(|\omega|)\right|^{2} \frac{2^{\ell} \tau^{-1 / 2}}{\sqrt{1-2^{2 \ell} \tau^{-1} \omega^{2}}} d \omega
\end{aligned}
$$

In a similar way as (3.33) we have that

$$
\begin{aligned}
\frac{\partial}{\partial \omega}\left\langle\xi, \nabla q_{\delta}\left(\cos \left(\theta_{\xi}+\arcsin \left(2^{\ell} \tau^{-1 / 2} \omega\right)\right), \sin \left(\theta_{\xi}+\arcsin \left(2^{\ell} \tau^{-1 / 2} \omega\right)\right)\right\rangle\right. \\
=\frac{62^{\ell} \tau^{-1 / 2}}{\sqrt{1-2^{2 \ell} \tau^{-1} \omega^{2}}} \delta|\xi| \frac{\cos \left(\theta_{\xi}+\arcsin \left(2^{\ell} \tau^{-1 / 2} \omega\right)\right)}{\sin ^{4}\left(\theta_{\xi}+\arcsin \left(2^{\ell} \tau^{-1 / 2} \omega\right)\right)} \\
\quad \times\left\langle\left(-\sin \left(\theta_{\xi}+\arcsin \left(2^{\ell} \tau^{-1 / 2} \omega\right)\right), \cos \left(\theta_{\xi}+\arcsin \left(2^{\ell} \tau^{-1 / 2} \omega\right)\right)\right), \frac{\xi}{|\xi|}\right\rangle \\
=\frac{62^{\ell} \tau^{-1 / 2} \delta|\xi|}{\sqrt{1-2^{2 \ell} \tau^{-1} \omega^{2}}} \frac{\cos \left(\theta_{\xi}+\arcsin \left(2^{\ell} \tau^{-1 / 2} \omega\right)\right)}{\sin ^{4}\left(\theta_{\xi}+\arcsin \left(2^{\ell} \tau^{-1 / 2} \omega\right)\right)}\left\langle\sin \left(\theta_{\xi}-\left(\theta_{\xi}+\arcsin \left(2^{\ell} \tau^{-1 / 2} \omega\right)\right)\right)\right\rangle \\
=\frac{-62^{2 \ell} \tau^{-1} \delta|\xi| \omega}{\sqrt{1-2^{2 \ell} \tau^{-1} \omega^{2}}} \frac{\cos \left(\theta_{\xi}+\arcsin \left(2^{\ell} \tau^{-1 / 2} \omega\right)\right)}{\sin ^{4}\left(\theta_{\xi}+\arcsin \left(2^{\ell} \tau^{-1 / 2} \omega\right)\right)}
\end{aligned}
$$

Since the support of $\rho$ and $\beta$ gives that $\theta_{\xi}+\arcsin \left(2^{\ell} \tau^{-1 / 2} \omega\right) \approx 1$ and $|\omega| \approx 1$, in addition to $|\xi| \approx \tau$ and $\ell \leq C \log _{2} \tau^{1 / 2}$, then

$$
\frac{\partial}{\partial \omega}\left\langle\xi, \nabla q_{\delta}\left(\cos \left(\theta_{\xi}+\arcsin \left(2^{\ell} \tau^{-1 / 2} \omega\right)\right), \sin \left(\theta_{\xi}+\arcsin \left(2^{\ell} \tau^{-1 / 2} \omega\right)\right)\right\rangle \approx 2^{2 \ell} \delta\right.
$$

The claim (3.36) will follow from integration by parts. Based on the above claim, 
Hölder's inequality and Plancherel's theorem, we have

$$
\begin{aligned}
& \iint\left|A_{\theta}^{\tau, \ell} g(y)\right|^{2} d \theta d y \\
& \leq C_{N} 2^{\ell} \tau^{-1 / 2} \int_{\mathbb{R}^{2}}\left\{\int_{0}^{1} \int_{0}^{1} \frac{\left|\overline{g\left(\hat{\xi}, t^{\prime}\right)}\right|}{\left(1+\delta 2^{2 \ell}\left|t-t^{\prime}\right|\right)^{N}} d t^{\prime}|g(\hat{\xi}, t)| d t\right\}\left|\beta\left(\frac{|\xi|}{\tau}\right) \sigma(\bar{\delta} \xi)\right|^{2} d \xi \\
& \leq C_{N} 2^{\ell} \tau^{-1 / 2} \int_{\mathbb{R}^{2}}\left\{\int_{0}^{1} \overline{|g(\hat{\xi}, \cdot)|} *\left(1+\delta 2^{2 \ell}|\cdot|\right)^{-N}(t)|g(\hat{\xi}, t)| d t\right\}\left|\beta\left(\frac{|\xi|}{\tau}\right) \sigma(\bar{\delta} \xi)\right|^{2} d \xi \\
& \leq C_{N} 2^{\ell} \tau^{-1 / 2} \int_{\mathbb{R}^{2}}\left\|\left.\left|\overline{|g(\hat{\xi}, \cdot)|} *\left(1+\delta 2^{2 \ell}|\cdot|\right)^{-N}\left\|_{\left(L^{2}, d t\right)}\right\| g(\hat{\xi}, t) \|_{\left(L^{2}, d t\right)}\right| \beta\left(\frac{|\xi|}{\tau}\right) \sigma(\bar{\delta} \xi)\right|^{2} d \xi\right. \\
& \leq C_{N} 2^{-\ell} \tau^{-1 / 2} \delta^{-1}\|g\|_{L^{2}}^{2},
\end{aligned}
$$

and inequality (3.34) has been proved for $\ell>0$.

For $\ell=0$, the proof is simpler. We still make the change of variable $\theta \rightarrow \theta_{\xi}+$ $\arcsin \left(\tau^{-1 / 2} \omega\right)$, then the support of the symbol of the operator $H^{0, \tau}$ gives that 0 is the non-degenerate critical point, since

$$
\begin{aligned}
& \left.\frac{\partial^{2}}{\partial \omega^{2}}\left\langle\xi, \nabla q_{\delta}\left(\cos \left(\theta_{\xi}+\arcsin \left(\tau^{-1 / 2} \omega\right)\right), \sin \left(\theta_{\xi}+\arcsin \left(\tau^{-1 / 2} \omega\right)\right)\right)\right\rangle\right|_{\omega=0} \\
& =-6 \delta|\xi| \tau^{-1} \frac{\cos \theta_{\xi}}{\sin ^{4} \theta_{\xi}} \approx \delta .
\end{aligned}
$$

Finally, integration by parts implies that

$$
\left|H^{0, \tau}\left(t, t^{\prime}, \xi\right)\right| \leq C \tau^{-1 / 2}\left(1+\delta\left|t-t^{\prime}\right|\right)^{-1 / 2}
$$

We still use Hölder's inequality and Plancherel's theorem to get inequality (3.34) for $\ell=0$. 


\section{Chapter 4}

\section{More general Fourier integral operators not satisfying}

\section{the cinematic curvature condition uniformly}

The purpose of this chapter is to prove Theorem 2.3.3. The main idea follows from the proof of Theorem 6.1 given in [26]. In fact, the proof has a similar structure with Chapter 3. Instead of repeating the proof here, we give a brief overview, and only a detailed argument partly. In this chapter, we still assume $\delta \lambda>1$ and the $\xi$-support of the symbol $a$ is in the first quadrant.

Since $t \approx 1$, we can replace $t$ by $1 / t$ in (2.65). By (2.38), we write

$$
-t^{-2} \xi_{2} \Phi(s, \tilde{q}(s, \delta), \delta):=E(\xi)+q_{\delta}(\xi, t), \quad s=s\left(\xi, t^{-1}\right),
$$

where

$$
E(\xi)=\frac{\xi_{1}^{2}}{2 \xi_{2}}, \quad q_{\delta}(\xi, t)=t \delta \phi^{\prime}(0) \frac{\xi_{1}^{3}}{\xi_{2}^{2}}+\delta^{2} R(t, \delta, \xi),
$$

by abuse of notation, we have written $R(t, \delta, \xi)$ in place of $R\left(t^{-1}, \delta, \xi\right)$ in (4.1).

Then

$$
\tilde{F}_{\lambda} f(y, t):=\tilde{F}_{\lambda}^{\delta} f(y, t)=\int_{\mathbb{R}^{2}} e^{i\left(\xi \cdot y+E(\xi)+q_{\delta}(\xi, t)\right.} \tilde{a}_{\lambda}(y, t, \xi) \hat{f}(\xi) d \xi,
$$

where $\tilde{a}_{\lambda}(y, t, \xi)=\rho_{1}(y, t) a(\xi, t) \rho_{0}\left(\frac{\delta}{\lambda}|\xi|\right) \tilde{\chi}\left(\frac{\xi_{1}}{\xi_{2}}\right)$, which is a symbol of order zero in $\xi$.

The support of $\tilde{\chi}$ implies that we are working in a fixed, small conic region $c_{1} \leq$ $\left|\xi_{1} / \xi_{2}\right| \leq c_{2}$. Using the angular decomposition appeared in Chapter 3, we can write 
$\tilde{F}_{\lambda}=\sum_{\nu} F_{\lambda}^{\nu}$, where the sum runs only over $\left(\frac{\pi}{2}-c_{2}\right) \sqrt{\frac{\lambda}{\delta}} \lesssim \nu \lesssim\left(\frac{\pi}{2}-c_{1}\right) \sqrt{\frac{\lambda}{\delta}}$ and

$$
\tilde{F}_{\lambda}^{\nu} f(y, t):=\int_{\mathbb{R}^{2}} e^{i\left(\xi \cdot y+E(\xi)+q_{\delta}(\xi, t)\right)} \tilde{a}_{\lambda}(y, t, \xi) \chi_{\nu}(\xi) \hat{f}(\xi) d \xi
$$

By Minkowski's inequality, we have

$$
\begin{aligned}
\left\|\tilde{F}_{\lambda} f\right\|_{L^{4}}^{2} & =\left\|\sum_{\nu, \mu} \tilde{F}_{\lambda}^{\nu} f \tilde{F}_{\lambda}^{\mu} f\right\|_{L^{2}} \\
& =\left\|\sum_{\ell} \sum_{|\nu-\mu| \approx 2^{\ell}} \tilde{F}_{\lambda}^{\nu} f \tilde{F}_{\lambda}^{\mu} f\right\|_{L^{2}} \\
& \leq C \sum_{\ell}\left\|\sum_{|\nu-\mu| \approx 2^{\ell}, \nu \leq \mu} \tilde{F}_{\lambda}^{\nu} f \tilde{F}_{\lambda}^{\mu} f\right\|_{L^{2}},
\end{aligned}
$$

where $2^{\ell} \leq\left(c_{2}-c_{1}\right) \sqrt{\frac{\lambda}{\delta}}$.

Now we make a further decomposition so that the symbol becomes

$$
\tilde{a}_{\lambda, \ell}^{\nu, j}(y, t, \xi)=\tilde{a}_{\lambda}(y, t, \xi) \chi_{\nu}(\xi) \varphi\left(2^{\ell} \lambda^{-1} q_{\delta}^{\prime}(\xi, t)-j\right)
$$

where $\varphi$ is defined as in Chapter 3 and $q_{\delta}^{\prime}(\xi, t)=\partial_{t} q_{\delta}(\xi, t)$. Since $|\xi| \approx \frac{\lambda}{\delta}$, then we have $\tilde{F}_{\lambda}^{\nu}=\sum_{j \approx 2^{\ell}} \tilde{F}_{\lambda, \ell}^{\nu, j}$, where

$$
\tilde{F}_{\lambda, \ell}^{\nu, j} f(y, t)=\int_{\mathbb{R}^{2}} e^{i\left(\xi \cdot y+E(\xi)+q_{\delta}(\xi, t)\right)} \tilde{a}_{\lambda, \ell}^{\nu, j}(y, t, \xi) \hat{f}(\xi) d \xi
$$

Since $c_{1} \leq\left|\xi_{1} / \xi_{2}\right| \leq c_{2}$ and $q_{\delta}^{\prime}(\xi, t)=\delta \phi^{\prime}(0) \frac{\xi_{1}^{3}}{\xi_{2}^{2}}+\delta^{2} \partial_{t} R(t, \delta, \xi)$, then $2^{-\ell} \lambda(j-1) \lesssim \delta|\xi| \lesssim$ $2^{-\ell} \lambda(j+1)$. The support of $\chi_{\nu}$ implies that the $\xi$-support of the symbol is comparable to a $2^{-\ell} \frac{\lambda}{\delta} \times\left(\frac{\lambda}{\delta}\right)^{1 / 2}$ rectangle. Meanwhile, by (3.9) and (4.1), we notice that

$$
\left|\partial_{\xi}^{\alpha} \partial_{y, t}^{\beta} \tilde{a}_{\lambda, \ell}^{\nu, j}(y, t, \xi)\right| \leq C_{\alpha, \beta}(1+|\xi|)^{-|\alpha| / 2}(\delta \lambda)^{|\beta| / 2} .
$$

Now we use two almost orthogonal lemmas. One of them is as follows

Lemma 4.0.9. Suppose that $\left|j+k-j^{\prime}-k^{\prime}\right| \geq \lambda^{\varepsilon}$. Then for any $N>0$,

$$
\left|\int_{\mathbb{R}^{3}} \tilde{F}_{\lambda, \ell}^{\nu, j} f \tilde{F}_{\lambda, \ell}^{\mu, k} f \overline{\tilde{F}_{\lambda, \ell}^{\nu^{\prime}, j^{\prime}} f \tilde{F}_{\lambda, \ell}^{\mu^{\prime}, k^{\prime}}} f d y d t\right| \leq C_{\varepsilon, N} \lambda^{-N}\|f\|_{L^{4}}^{4}
$$

Proof.

$$
\int_{\mathbb{R}^{3}} \tilde{F}_{\lambda, \ell}^{\nu, j} f \tilde{F}_{\lambda, \ell}^{\mu, k} f \overline{\tilde{F}_{\lambda, \ell}^{\nu^{\prime}, j^{\prime}} f \tilde{F}_{\lambda, \ell}^{\mu^{\prime}, k^{\prime}} f} d y d t=\int H_{j, k, j^{\prime}, k^{\prime}}^{\nu, \mu, \nu^{\prime}, \mu^{\prime}}\left(\eta, \xi, \eta^{\prime}, \xi^{\prime}\right) \hat{f}(\eta) \hat{f}(\xi) \overline{\hat{f}\left(\eta^{\prime}\right) \hat{f}\left(\xi^{\prime}\right)} d \eta d \xi d \eta^{\prime} d \xi^{\prime}
$$


where

$$
H_{j, k, j^{\prime}, k^{\prime}}^{\nu, \mu, \nu^{\prime}, \mu^{\prime}}\left(\eta, \xi, \eta^{\prime}, \xi^{\prime}\right)=\int_{\mathbb{R}^{3}} e^{i \Psi\left(y, t, \eta, \xi, \eta^{\prime}, \xi^{\prime}\right)} b_{j, k, j^{\prime}, k^{\prime}}^{\nu, \mu, \nu^{\prime}, \mu^{\prime}}\left(y, t, \eta, \xi, \eta^{\prime}, \xi^{\prime}\right) d y d t
$$

$\Psi\left(y, t, \eta, \xi, \eta^{\prime}, \xi^{\prime}\right)=y \cdot\left(\eta+\xi-\eta^{\prime}-\xi^{\prime}\right)+\frac{1}{2}\left(\frac{\eta_{1}^{2}}{\eta_{2}}+\frac{\xi_{1}^{2}}{\xi_{2}}-\frac{\eta_{1}^{\prime 2}}{\eta_{2}^{\prime}}-\frac{\eta_{1}^{\prime 2}}{\eta_{2}^{\prime}}\right)+F\left(t, \eta, \xi, \eta^{\prime}, \xi^{\prime}\right)$, $F\left(t, \eta, \xi, \eta^{\prime}, \xi^{\prime}\right)=q_{\delta}(\eta, t)+q_{\delta}(\xi, t)-q_{\delta}\left(\eta^{\prime}, t\right)-q_{\delta}\left(\xi^{\prime}, t\right)$ and

$$
b_{j, k, j^{\prime}, k^{\prime}}^{\nu, \mu, \nu^{\prime}, \mu^{\prime}}\left(y, t, \eta, \xi, \eta^{\prime}, \xi^{\prime}\right)=\tilde{a}_{\lambda, \ell}^{\nu, j}(y, t, \eta) \tilde{a}_{\lambda, \ell}^{\mu, k}(y, t, \xi) \overline{\tilde{a}_{\lambda, \ell}^{\nu^{\prime}, j^{\prime}}\left(y, t, \eta^{\prime}\right) \tilde{a}_{\lambda, \ell}^{\mu^{\prime}, k^{\prime}}\left(y, t, \xi^{\prime}\right)} .
$$

Let $\mathcal{L} g=\frac{\partial}{\partial_{t}}\left(\frac{g}{\partial_{t} \Psi}\right)$. By integration by parts, we have

$$
\left|H_{j, k, j^{\prime}, k^{\prime}}^{\nu, \mu, \nu^{\prime}, \mu^{\prime}}\left(\eta, \xi, \eta^{\prime}, \xi^{\prime}\right)\right| \leq \int_{\mathbb{R}^{3}}\left|\mathcal{L}^{N} b_{j, k, j^{\prime}, k^{\prime}}^{\nu, \mu, \nu^{\prime}, \mu^{\prime}}\left(y, t, \eta, \xi, \eta^{\prime}, \xi^{\prime}\right)\right| d y d t
$$

Since $\partial_{t} \Psi=\partial_{t} F, \mathcal{L} g=\frac{\partial}{\partial_{t}}\left(\frac{g}{\partial_{t} F}\right)$, then the inner integral can be written as the sum of expressions of the form

$$
\frac{\prod_{i=0}^{N_{2}}\left(\Psi_{i}(t, \eta)+\Psi_{i}(t, \xi)-\Psi_{i}\left(t, \eta^{\prime}\right)-\Psi_{i}\left(t, \xi^{\prime}\right)\right)}{\left(\partial_{t} F\right)^{N+N_{1}}} \partial_{t}^{\beta} b_{j, k, j^{\prime}, k^{\prime}}^{\nu, \mu, \nu^{\prime}, \mu^{\prime}}\left(y, t, \eta, \xi, \eta^{\prime}, \xi^{\prime}\right),
$$

where $0 \leq N_{1} \leq N, 0 \leq N_{2} \leq N_{1}, \beta \leq N-N_{2}$, and $\Psi_{i}$ are partial derivative of $\partial_{t} F$ with respect to $t$-variable. Note that $\Psi_{i}$ are still homogeneous of degree one in $\xi$. Now from the estimate (4.5) and the support of $\varphi$, it is easy to see that

$$
\left|\partial_{t} F\left(t, \eta, \xi, \eta^{\prime}, \xi^{\prime}\right)\right| \geq C 2^{-\ell} \lambda\left|j+k-j^{\prime}-k^{\prime}\right| \geq C(\delta \lambda)^{1 / 2}\left|j+k-j^{\prime}-k^{\prime}\right| .
$$

Since $\left|\Psi_{i}(t, \cdot)\right| \leq\left|\partial_{t}^{2+\alpha} q_{\delta}(\cdot, t)\right| \leq \delta^{2} \frac{\lambda}{\delta}=\delta \lambda$ for all $\alpha \geq 0$, then

$$
\left|\prod_{i=0}^{N_{2}}\left(\Psi_{i}(t, \eta)+\Psi_{i}(t, \xi)-\Psi_{i}\left(t, \eta^{\prime}\right)-\Psi_{i}\left(t, \xi^{\prime}\right)\right)\right| \leq C(\delta \lambda)^{N_{2}+1},
$$

in addition to the fact that

$$
\left|\partial_{t} b_{j, k, j^{\prime}, k^{\prime}}^{\nu, \mu, \nu^{\prime}, \mu^{\prime}}\right| \leq 2^{\ell} \lambda^{-1} \delta^{2} \frac{\lambda}{\delta}=2^{\ell} \delta \leq\left(\frac{\lambda}{\delta}\right)^{1 / 2} \delta=(\delta \lambda)^{1 / 2}
$$

we have

$$
\left|H_{j, k, j^{\prime}, k^{\prime}}^{\nu, \mu, \nu^{\prime}, \mu^{\prime}}\left(y, \eta, \xi, \eta^{\prime}, \xi^{\prime}\right)\right| \leq C \frac{(\delta \lambda)^{N_{2}+1}(\delta \lambda)^{\left(N-N_{2}\right) / 2}}{\left((\delta \lambda)^{1 / 2}\left|j+k-j^{\prime}-k^{\prime}\right|\right)^{N+N_{1}}} \leq C \lambda^{-\varepsilon N}(\delta \lambda),
$$

provided that $\left|j+k-j^{\prime}-k^{\prime}\right| \geq \lambda^{\varepsilon}$. Since $b_{j, k, j^{\prime}, k^{\prime}}^{\nu, \mu, \nu^{\prime}, \mu^{\prime}}$ is supported on a set of measure $2^{-4 \ell}\left(\frac{\lambda}{\delta}\right)^{6}$ and $\delta^{-1}<\lambda$, then the integral is bounded by $C_{\varepsilon, N} \lambda^{-N}\|f\|_{L^{1}}^{4}$, in addition to the compact support of $f$, we get the desired result. 
The above lemma implies that

$$
\begin{aligned}
& \int\left|\sum_{|\nu-\mu| \approx 2^{\ell}} \tilde{F}_{\lambda}^{\nu} f \tilde{F}_{\lambda}^{\mu} f\right|^{2} d y d t \\
& \leq \int\left|\sum_{|\nu-\mu| \approx 2^{\ell}} \sum_{j, k \leq 2^{\ell}} \tilde{F}_{\lambda, \ell}^{\nu, j} f \tilde{F}_{\lambda, \ell}^{\mu, k} f\right|^{2} d y d t \\
& =\int\left|\sum_{s \leq 2^{\ell+1}} \sum_{j+k=s} \sum_{|\nu-\mu| \approx 2^{\ell}} \tilde{F}_{\lambda, \ell}^{\nu, j} f \tilde{F}_{\lambda, \ell}^{\mu, k} f\right|^{2} d y d t \\
& =\int\left|\sum_{\substack{s \leq 2^{\ell+1} \\
s^{\prime} \leq 2^{\ell+1}}} \sum_{\substack{j+k=s \\
j^{\prime}+k^{\prime}=s^{\prime}}} \sum_{\substack{|\nu-\mu| \approx 2^{\ell} \\
\left|\nu^{\prime}-\mu^{\prime}\right| \approx 2^{\ell}}} \tilde{F}_{\lambda, \ell}^{\nu, j} f \tilde{F}_{\lambda, \ell}^{\mu, k} f \overline{\tilde{F}_{\lambda, \ell}^{\nu^{\prime}, j^{\prime}} f \tilde{F}_{\lambda, \ell}^{\mu^{\prime}, k^{\prime}} f}\right| d y d t \\
& \leq \int\left|\sum_{s, s^{\prime},\left|s-s^{\prime}\right| \leq \lambda^{\varepsilon}} \sum_{\substack{j+k=s \\
j^{\prime}+k^{\prime}=s^{\prime}}} \sum_{\substack{|\nu-\mu| \approx 2^{\ell} \\
\left|\nu^{\prime}-\mu^{\prime}\right| \approx 2^{\ell}}} \tilde{F}_{\lambda, \ell}^{\nu, j} f \tilde{F}_{\lambda, \ell}^{\mu, k} f \tilde{F}_{\lambda, \ell}^{\nu^{\prime}, j^{\prime}} f \tilde{F}_{\lambda, \ell}^{\mu^{\prime}, k^{\prime}} f\right| d y d t+C \lambda^{-N}\|f\|_{L^{4}}^{4} .
\end{aligned}
$$

By the Cauchy-Schwarz inequality, we have that

$$
\begin{aligned}
& \int\left|\sum_{s, s^{\prime},\left|s-s^{\prime}\right| \leq \lambda^{\varepsilon}} \sum_{\substack{j+k=s \\
j^{\prime}+k^{\prime}=s^{\prime}}} \sum_{\substack{|\nu-\mu| \approx 2^{\ell} \\
\left|\nu^{\prime}-\mu^{\prime}\right| \approx 2^{\ell}}} \tilde{F}_{\lambda, \ell}^{\nu, j} f \tilde{F}_{\lambda, \ell}^{\mu, k} f \overline{\tilde{F}_{\lambda, \ell}^{\nu^{\prime}, j^{\prime}} f \tilde{F}_{\lambda, \ell}^{\mu^{\prime}, k^{\prime}} f}\right| d y d t \\
& \leq \lambda^{\varepsilon} \sum_{s \leq 2^{\ell+1}} \int\left|\sum_{\substack{j+k=s \\
j^{\prime}+k^{\prime}=s}} \sum_{\substack{|\nu-\mu| \approx 2^{\ell} \\
\left|\nu^{\prime}-\mu^{\prime}\right| \approx 2^{\ell}}} \tilde{F}_{\lambda, \ell}^{\nu, j} f \tilde{F}_{\lambda, \ell}^{\mu, k} f \overline{\tilde{F}_{\lambda, \ell}^{\nu^{\prime}, j^{\prime}} f \tilde{F}_{\lambda, \ell}^{\mu^{\prime}, k^{\prime}} f}\right| d y d t \\
& =\lambda^{\varepsilon} \sum_{s \leq 2^{\ell+1}} \int\left|\sum_{j+k=s} \sum_{|\nu-\mu| \approx 2^{\ell}} \tilde{F}_{\lambda, \ell}^{\nu, j} f \tilde{F}_{\lambda, \ell}^{\mu, k} f\right|^{2} d y d t \\
& \leq C \lambda^{\varepsilon} 2^{\ell+1} \sum_{s \leq 2^{\ell+1}} \int \sum_{j+k=s}\left|\sum_{\substack{|\nu-\mu| \approx 2^{\ell} \\
j, \ell}} \tilde{F}_{\lambda, \ell}^{\nu, j} f \tilde{F}_{\lambda, \ell}^{\mu, k} f\right|^{2} d y d t \\
& \leq C \lambda^{\varepsilon} 2^{\ell+1}||\left(\sum_{j, k}\left|\sum_{|\nu-\mu| \approx 2^{\ell}} \tilde{F}_{\lambda, \ell}^{\nu, j} f \tilde{F}_{\lambda, \ell}^{\mu, k} f\right|^{2}\right)^{1 / 2} \|_{L^{2}}^{2} .
\end{aligned}
$$

Now we use another almost orthogonality lemma to bring the sum in $\mu$ and $\nu$ outside of the square function.

Lemma 4.0.10. Suppose that $|\nu-\mu| \approx 2^{\ell},\left|\nu^{\prime}-\mu^{\prime}\right| \approx 2^{\ell}$, and $\left|\nu-\nu^{\prime}\right|+\left|\mu-\mu^{\prime}\right| \geq \lambda^{\varepsilon}$. Then for any $N>0$,

$$
\left|\int_{\mathbb{R}^{3}} \tilde{F}_{\lambda, \ell}^{\nu, j} f \tilde{F}_{\lambda, \ell}^{\mu, k} f \overline{\tilde{F}_{\lambda, \ell}^{\nu^{\prime}, j^{\prime}} f \tilde{F}_{\lambda, \ell}^{\mu^{\prime}, k^{\prime}}} f d y d t\right| \leq C_{\varepsilon, N} \lambda^{-N}\|f\|_{L^{4}}^{4}
$$


Proof. First we will introduce Lemma 6.8 in [26] as follows.

Lemma 4.0.11. [26] Let $\tilde{\Psi} \in C^{4}\left(\mathbb{R}^{2} \backslash 0\right)$ be homogeneous of degree one. Let $\varsigma<\pi / 4$, $a_{0}<1 / 4, A_{0} \geq 1$. Let $\mathcal{S}_{\lambda}$ be the intersection of a sector which subtends an angle of size $\varsigma$ with the annulus $\left\{\eta:\left(1-a_{0}\right) \lambda \leq|\eta| \leq\left(1+a_{0}\right) \lambda\right\}$. Let $h \in C^{1}\left(\mathbb{R}^{2} \backslash 0\right)$ be homogeneous of degree one such that $b_{0}|\eta| \leq h(\eta) \leq b_{1}|\eta|,\left|\nabla_{\eta} h(\eta)\right| \leq b_{2}$ for some positive constants $b_{0}, b_{1}, b_{2}$.

Suppose that $a_{0}^{-1} \leq 2^{n}, n \leq \ell, 2^{\ell} \leq \varsigma \lambda^{1 / 2}$, and that $\eta, \xi, \eta^{\prime}, \xi^{\prime} \in \mathcal{S}_{\lambda}$ are chosen such that for given integers $\nu, \mu, \nu^{\prime}, \mu^{\prime}$

(1) $\left|\arg (\eta)-\nu \lambda^{-1 / 2}\right| \leq \lambda^{-1 / 2} ;\left|\arg \left(\eta^{\prime}\right)-\nu^{\prime} \lambda^{-1 / 2}\right| \leq \lambda^{-1 / 2}$;

(2) $\left|\arg (\eta)-\nu \lambda^{-1 / 2}\right| \leq \lambda^{-1 / 2} ;\left|\arg \left(\eta^{\prime}\right)-\nu^{\prime} \lambda^{-1 / 2}\right| \leq \lambda^{-1 / 2}$;

(3) $2^{\ell-1} \lambda^{-1 / 2} \leq \max \left\{|\arg (\eta)-\arg (\xi)|,\left|\arg \left(\eta^{\prime}\right)-\arg \left(\xi^{\prime}\right)\right|\right\} \leq 2^{\ell+1} \lambda^{-1 / 2}$;

(4) $\left|h(\eta)-h\left(\eta^{\prime}\right)\right| \leq 2^{-n} \lambda$;

(5) $\left|h(\xi)-h\left(\xi^{\prime}\right)\right| \leq 2^{-n} \lambda$.

Then one can choose $\varsigma, a_{0}$ sufficiently small, and $A_{0}$ sufficiently large (only depending on $\left.\tilde{\Psi}, b_{0}, b_{1}, b_{2}\right)$ such that for all $\nu, \mu, \nu^{\prime}, \mu^{\prime}$ with $A_{0} 2^{\ell-n} \leq\left|\nu-\nu^{\prime}\right|+\left|\mu-\mu^{\prime}\right| \leq \varsigma \lambda^{1 / 2}$

$$
\begin{aligned}
\mid \tilde{\Psi}(\eta)+ & \tilde{\Psi}(\xi)-\tilde{\Psi}\left(\eta^{\prime}\right)-\tilde{\Psi}\left(\xi^{\prime}\right) \mid \\
& \leq C\left[\left(2^{\ell}\left|\nu-\nu^{\prime}\right|+\left|\nu-\nu^{\prime}\right|^{2}\right)+\left(2^{\ell}\left|\mu-\mu^{\prime}\right|+\left|\mu-\mu^{\prime}\right|^{2}\right)+\left|\eta+\xi-\eta^{\prime}-\xi^{\prime}\right|\right] .
\end{aligned}
$$

Suppose now that $\tilde{\Psi}$ satisfies the additional assumption rank $\tilde{\Psi}_{\eta \eta}^{\prime \prime}=1$. Then if either $\mu \leq \nu$ and $\mu^{\prime} \leq \nu^{\prime}$ or $\nu \leq \mu$ and $\nu^{\prime} \leq \mu^{\prime}$ and if $A_{0} 2^{\ell-n} \leq\left|\nu-\nu^{\prime}\right|+\left|\mu-\mu^{\prime}\right| \leq \varsigma \lambda^{1 / 2}$ we have also with suitable positive constants $c_{0}, C_{0}$

$$
\begin{aligned}
\left|\tilde{\Psi}(\eta)+\tilde{\Psi}(\xi)-\tilde{\Psi}\left(\eta^{\prime}\right)-\tilde{\Psi}\left(\xi^{\prime}\right)\right| \\
\quad \geq c_{0}\left[\left(2^{\ell}\left|\nu-\nu^{\prime}\right|+\left|\nu-\nu^{\prime}\right|^{2}\right)+\left(2^{\ell}\left|\mu-\mu^{\prime}\right|+\left|\mu-\mu^{\prime}\right|^{2}\right)\right]-C_{0}\left|\eta+\xi-\eta^{\prime}-\xi^{\prime}\right|
\end{aligned}
$$

Now in order to apply the above lemma to evaluate $(4.11)$, we rewrite $(4.11)$ as $\frac{\delta^{2\left(N_{2}+1\right)}}{\delta^{N+N_{1}}}$ times

$$
\frac{\prod_{i=0}^{N_{2}}\left(\Psi_{i}(t, \eta) / \delta^{2}+\Psi_{i}(t, \xi) / \delta^{2}-\Psi_{i}\left(t, \eta^{\prime}\right) / \delta^{2}-\Psi_{i}\left(t, \xi^{\prime}\right) / \delta^{2}\right)}{\left(\partial_{t} F / \delta\right)^{N+N_{1}}} \partial_{t}^{\beta} b_{j, k, j^{\prime}, k^{\prime}}^{\nu, \mu, \nu^{\prime}, \mu^{\prime}}\left(y, t, \eta, \xi, \eta^{\prime}, \xi^{\prime}\right) .
$$

The worst case to bound the numerator of (4.18) from above is that we apply inequality (4.16) in the above lemma with $\tilde{\Psi}=\frac{1}{\delta^{2}} \partial_{t}^{2} q_{\delta}(\cdot, t)$, at the same time, we will replace $\varsigma$ by $c_{2}-c_{1}, h(\eta)$ by $\frac{1}{\delta^{2}} \partial_{t}^{2} q_{\delta}(\eta, t)$ and $\lambda$ by $\lambda / \delta$ in the above lemma. In order to bound the denominator of (4.18) from below, we apply inequality (4.17) in the above lemma with $\tilde{\Psi}=\frac{1}{\delta} q_{\delta}^{\prime}$, in this case, we will replace $\varsigma$ by $c_{2}-c_{1}, h(\eta)$ by $\frac{1}{\delta} q_{\delta}^{\prime}(\eta, t)$ and $\lambda$ by $\lambda / \delta$ in 
the above lemma. Note that $\partial_{y} \Psi\left(y, t, \eta, \xi, \eta^{\prime}, \xi^{\prime}\right)=\eta+\xi-\eta^{\prime}-\xi^{\prime}$, by $(4.12), \frac{\delta^{2\left(N_{2}+1\right)}}{\delta^{N+N_{1}}} \times$ (4.18) can be controlled by

$$
\frac{C \delta^{N_{2}+1}(\delta \lambda)^{\left(N-N_{2}\right) / 2}\left[\delta\left(\left(2^{\ell}\left|\nu-\nu^{\prime}\right|+\left|\nu-\nu^{\prime}\right|^{2}\right)+\left(2^{\ell}\left|\mu-\mu^{\prime}\right|+\left|\mu-\mu^{\prime}\right|^{2}\right)+\left|\partial_{y} \Psi\right|\right)\right]^{N_{2}+1}}{\left(\delta \max \left\{\left|\partial_{y} \Psi\right|,\left|\left(2^{\ell}\left|\nu-\nu^{\prime}\right|+\left|\nu-\nu^{\prime}\right|^{2}\right)+\left(2^{\ell}\left|\mu-\mu^{\prime}\right|+\left|\mu-\mu^{\prime}\right|^{2}\right)-\right| \partial_{y} \Psi||\right\}\right)^{N+N_{1}}}
$$

If $\left|\nu-\nu^{\prime}\right|+\left|\mu-\mu^{\prime}\right| \geq \lambda^{\varepsilon}$, together with the fact that $\left(\frac{\pi}{2}-c_{2}\right) \sqrt{\frac{\lambda}{\delta}} \lesssim \nu \lesssim\left(\frac{\pi}{2}-c_{1}\right) \sqrt{\frac{\lambda}{\delta}}$, then

$$
\begin{aligned}
& \frac{1}{\delta\left(\left(2^{\ell}\left|\nu-\nu^{\prime}\right|+\left|\nu-\nu^{\prime}\right|^{2}\right)+\left(2^{\ell}\left|\mu-\mu^{\prime}\right|+\left|\mu-\mu^{\prime}\right|^{2}\right)\right)} \\
& \leq C \delta^{-1}\left(2^{\ell} \lambda^{\epsilon}+\sqrt{\frac{\lambda}{\delta}} \lambda^{\epsilon}\right)^{-1} \\
& \leq C(\delta \lambda)^{-1 / 2} \lambda^{-\epsilon} .
\end{aligned}
$$

Since $\delta \lambda>1$, (4.19) can be controlled by $\mathcal{O}\left(\frac{\delta^{N_{2}+1}(\delta \lambda)^{\left(N-N_{2}\right) / 2}}{(\delta \lambda)^{(N-1) / 2} \lambda^{\varepsilon(N-1)}}\right)=\mathcal{O}\left(\frac{1}{\lambda^{\varepsilon(N-1)-1 / 2}}\right)$. Now if we choose $N$ sufficiently large, the similar argument with Lemma 4.0.9 gives the desired result.

Let us continue the proof of our theorem. Based on Lemma 4.0.10 and the CauchySchwarz inequality, then we have that

$$
\begin{aligned}
& \left\|\left(\sum_{j, k}\left|\sum_{|\nu-\mu| \approx 2^{\ell}} \tilde{F}_{\lambda, \ell}^{\nu, j} f \tilde{F}_{\lambda, \ell}^{\mu, k} f\right|^{2}\right)^{1 / 2}\right\|_{L^{2}}^{2} \\
& =\int \sum_{j, k}\left|\sum_{\substack{|\nu-\mu| \approx \approx^{\ell} \\
\left|\nu^{\prime}-\mu^{\prime}\right| \approx 2^{\ell}}} \tilde{F}_{\lambda, \ell}^{\nu, j} f \tilde{F}_{\lambda, \ell}^{\mu, k} f \overline{\tilde{F}_{\lambda, \ell}^{\nu^{\prime}, j} f \tilde{F}_{\lambda, \ell}^{\mu^{\prime}, k} f}\right| d y d t \\
& \leq \int \sum_{j, k}\left|\sum_{\substack{|\nu-\mu|,\left|\nu^{\prime}-\mu^{\prime}\right| \approx 2^{\ell} \\
\left|\nu-\nu^{\prime}\right|+\left|\mu-\mu^{\prime}\right| \leq \lambda^{\varepsilon}}} \tilde{F}_{\lambda, \ell}^{\nu, j} f \tilde{F}_{\lambda, \ell}^{\mu, k} f \overline{\tilde{F}_{\lambda, \ell}^{\nu^{\prime}, j} f \tilde{F}_{\lambda, \ell}^{\mu^{\prime}, k} f}\right| d y d t+C \lambda^{-N}\|f\|_{L^{4}}^{4} \\
& \leq \lambda^{2 \varepsilon} \int \sum_{j, k} \sum_{|\nu-\mu| \approx 2^{\ell} \mid}\left|\tilde{F}_{\lambda, \ell}^{\nu, j} f \tilde{F}_{\lambda, \ell}^{\mu, k} f\right|^{2} d y d t+C \lambda^{-N}\|f\|_{L^{4}}^{4} \\
& \leq \lambda^{2 \varepsilon}\left\|\left(\sum_{j, \nu}\left|\tilde{F}_{\lambda, \ell}^{\nu, j} f\right|^{2}\right)^{1 / 2}\right\|_{L^{4}}^{4}+C \lambda^{-N}\|f\|_{L^{4}}^{4} .
\end{aligned}
$$

At present, we put all estimates together and get

$$
\left\|\tilde{F}_{\lambda} f\right\|_{L^{4}}^{2} \leq C \lambda^{\varepsilon} \sum_{\ell} 2^{\ell / 2}\left\|\left(\sum_{j, \nu}\left|\tilde{F}_{\lambda, \ell}^{\nu, j} f\right|^{2}\right)^{1 / 2}\right\|_{L^{4}}^{2}+C \lambda^{-N}\|f\|_{L^{4}}^{2}
$$


Furthermore, we introduce a finer decomposition of the operators $\tilde{F}_{\lambda, \ell}^{\nu, j}$, namely, we set

$$
\tilde{F}_{\lambda, \ell}^{\nu, j, n} f(y, t):=\int_{\mathbb{R}^{2}} e^{i\left(\xi \cdot y+E(\xi)+q_{\delta}(\xi, t)\right)} \tilde{a}_{\lambda, \ell}^{\nu, j, n}(y, t, \xi) \hat{f}(\xi) d \xi
$$

where

$$
\tilde{a}_{\lambda, \ell}^{\nu, j, n}(y, t, \xi)=\varphi\left(\lambda^{-1 / 2} q_{\delta}^{\prime}(\xi, t)-n\right) \tilde{a}_{\lambda, \ell}^{\nu, j}(y, t, \xi)
$$

For fixed $y, t, \operatorname{supp} \xi \rightarrow \tilde{a}_{\lambda, \ell}^{\nu, j, n}$ is comparable to a $\frac{\lambda^{1 / 2}}{\delta} \times\left(\frac{\lambda}{\delta}\right)^{1 / 2}$ rectangle and $\tilde{F}_{\lambda, \ell}^{\nu, j}=$ $\sum_{n} \tilde{F}_{\lambda, \ell}^{\nu, j, n}$, where the sum involves less than $2^{-\ell} \lambda^{1 / 2}$. Since on the support of the symbols $\tilde{a}_{\lambda, \ell}^{\nu, j, n}, \lambda^{1 / 2}(n-1) \leq q_{\delta}^{\prime}(\xi, t) \leq \lambda^{1 / 2}(n+1)$ and on the support of the symbols $\tilde{a}_{\lambda, \ell}^{\nu, j}, 2^{-\ell} \lambda(j-1) \leq q_{\delta}^{\prime}(\xi, t) \leq 2^{-\ell} \lambda(j+1)$, for fixed $j$, then which implies that if $n$ satisfies $|n-n(j)| \leq 2^{-\ell} \lambda^{1 / 2}$ with $n(j)=\left[2^{-\ell} \lambda^{1 / 2} j\right]$, then $\tilde{F}_{\lambda, \ell}^{\nu, j, n} \neq 0$, where [.] denotes the nearest integer function.

One can follow the proof of Lemma 4.0.9 and get an almost orthogonality lemma similar to Lemma 4.0.9:

Lemma 4.0.12. Suppose that $\left|m+n-m^{\prime}-n^{\prime}\right| \geq \lambda^{\varepsilon}$. Then for any $N>0$,

$$
\left|\int_{\mathbb{R}^{3}} \tilde{F}_{\lambda, \ell}^{\nu, j, m} f \tilde{F}_{\lambda, \ell}^{\mu, k, n} f \overline{\tilde{F}_{\lambda, \ell}^{\nu, j, m^{\prime}} f \tilde{F}_{\lambda, \ell}^{\mu, k, n^{\prime}} f} d y d t\right| \leq C_{\varepsilon, N} \lambda^{-N}\|f\|_{L^{4}}^{4}
$$

Using this lemma and the Cauchy-Schwarz inequality, we obtain

$$
\begin{aligned}
& \left\|\left(\sum_{j, \nu}\left|\tilde{F}_{\lambda, \ell}^{\nu, j} f\right|^{2}\right)^{1 / 2}\right\|_{L^{4}}^{4} \\
& =\sum_{\substack{\nu, \mu \\
j, k}} \int\left|\sum_{m, n} \tilde{F}_{\lambda, \ell}^{\nu, j, m} f \tilde{F}_{\lambda, \ell}^{\mu, k, n} f\right|^{2} d y d t \\
& =\sum_{\substack{\nu, \mu \\
j, k}} \int\left|\sum_{\substack{s \leq 2^{-\ell} \lambda^{1 / 2} \\
s^{\prime} \leq 2^{-\ell} \lambda^{1 / 2}}} \sum_{\substack{m-m^{\prime}=s \\
n^{\prime}-n=s^{\prime}}} \tilde{F}_{\lambda, \ell}^{\nu, j, m} f \tilde{F}_{\lambda, \ell}^{\mu, k, n} f \overline{\tilde{F}_{\lambda, \ell}^{\nu, j, m^{\prime}} f \tilde{F}_{\lambda, \ell}^{\mu, k, n^{\prime}} f}\right| d y d t \\
& \leq \sum_{\substack{\nu, \mu \\
j, k}} \int\left|\sum_{\substack{s \leq 2^{-\ell} \lambda^{1 / 2} \\
s^{\prime} \leq 2^{-\ell} \lambda^{1 / 2} \\
\left|s-s^{\prime}\right| \leq \lambda^{\epsilon} \\
n^{\prime}-n=m^{\prime}=s}} \tilde{F}_{\lambda, \ell}^{\nu, j, m} f \tilde{F}_{\lambda, \ell}^{\mu, k, n} f \overline{\tilde{F}_{\lambda, \ell}^{\nu, j, m^{\prime}} f \tilde{F}_{\lambda, \ell}^{\mu, k, n^{\prime}} f}\right| d y d t \\
& \quad+C_{N} \lambda^{-N}\|f\|_{L^{4}}^{4}
\end{aligned}
$$


Chapter 4. More general Fourier integral operators not satisfying the cinematic curvature condition uniformly

$$
\begin{aligned}
& \leq \lambda^{\epsilon} \sum_{\substack{\nu, \mu \\
j, k}} \sum_{\substack{\leq \leq 2^{-\ell} \lambda^{1 / 2}\\
}} \int\left|\sum_{\substack{m-m^{\prime}=s \\
n^{\prime}-n=s}} \tilde{F}_{\lambda, \ell}^{\nu, j, m} f \tilde{F}_{\lambda, \ell}^{\mu, k, n} f \overline{\tilde{F}_{\lambda, \ell}^{\nu, j, m^{\prime}} f \tilde{F}_{\lambda, \ell}^{\mu, k, n^{\prime}} f}\right| d y d t \\
& =\lambda^{\epsilon} \sum_{\substack{\nu, \mu \\
j, k}} \sum_{s \leq 2^{-\ell} \lambda^{1 / 2}} \int\left|\sum_{m-m^{\prime}=s} \tilde{F}_{\lambda, \ell}^{\nu, j, m} f \overline{\tilde{F}_{\lambda, \ell}^{\nu, j, m^{\prime}} f} \sum_{n-n^{\prime}=s} \overline{\tilde{F}_{\lambda, \ell}^{\mu, k, n} f \overline{\tilde{F}_{\lambda, \ell}^{\mu, k, n^{\prime}} f}}\right| d y d t \\
& \leq \lambda^{\epsilon} \sum_{\substack{\nu, \mu \\
j, k}} \int\left(\sum_{s \leq 2^{-\ell} \lambda^{1 / 2}}\left|\sum_{m-m^{\prime}=s} \tilde{F}_{\lambda, \ell}^{\nu, j, m} f \overline{\tilde{F}_{\lambda, \ell}^{\nu, j, m^{\prime}} f}\right|^{2}\right)^{1 / 2} \\
& \times\left(\sum_{s \leq 2^{-\ell} \lambda^{1 / 2}}\left|\sum_{n-n^{\prime}=s} \tilde{F}_{\lambda, \ell}^{\mu, k, n} f \overline{\tilde{F}_{\lambda, \ell}^{\mu, k, n^{\prime}}} f\right|^{2}\right)^{1 / 2} d y d t \\
& =\lambda^{\varepsilon} \int\left(\sum_{j, \nu}\left(\sum_{s \leq 2^{-\ell} \lambda^{1 / 2}}\left|\sum_{n-n^{\prime}=s} \tilde{F}_{\lambda, \ell}^{\nu, j, n} f \overline{\tilde{F}_{\lambda, \ell}^{\nu, j, n^{\prime}} f}\right|^{2}\right)^{1 / 2}\right)^{2} d y d t \\
& \leq C \lambda^{\varepsilon} 2^{-\ell} \lambda^{1 / 2} \int\left(\sum_{j, \nu}\left(\sum_{s \leq 2^{-\ell} \lambda^{1 / 2}} \sum_{n-n^{\prime}=s}\left|\tilde{F}_{\lambda, \ell}^{j, \nu, n} f \overline{\tilde{F}_{\lambda, \ell}^{\nu, j, n^{\prime}} f}\right|^{2}\right)^{1 / 2}\right)^{2} d y d t \\
& \leq C \lambda^{\varepsilon} 2^{-\ell} \lambda^{1 / 2} \int\left(\sum_{j, \nu}\left(\sum_{n}\left|\tilde{F}_{\lambda, \ell}^{\nu, j, n} f\right|^{4}\right)^{1 / 2}\right)^{2} d y d t \\
& \leq C \lambda^{\varepsilon} 2^{-\ell} \lambda^{1 / 2}\left\|\left(\sum_{j, \nu, n}\left|\tilde{F}_{\lambda, \ell, n}^{\nu, j} f\right|^{2}\right)^{1 / 2}\right\|_{L^{4}}^{4}
\end{aligned}
$$

We would be done if we could prove that

$$
\left\|\left(\sum_{j, \nu, n}\left|\tilde{F}_{\lambda, \ell}^{\nu, j, n} f\right|^{2}\right)^{1 / 2}\right\|_{L^{4}} \leq C \delta^{-\left(1 / 2+\varepsilon_{2}\right)} \lambda^{\varepsilon_{1}}\|f\|_{L^{4}} .
$$

Now comparing expressions of $\tilde{F}_{\lambda, \ell}^{\nu, j, n} f$ and $\mathcal{F}_{\lambda, \nu}^{\delta} f_{n}$ which appeared in Chapter 3, we see that they have a similar support in $\xi$, see Figure 8 . Since all estimates after (3.0.6) in Chapter 3 are valid under small smooth perturbations $q_{\delta}^{\prime}$, then from now on, we can combine the idea from Chapter 3 and the proof of Theorem 6.1 in [26], the $L^{1}$ boundness on the kernel of the remaining operator, Carleson's square function estimate, and a Kakeya-type maximal estimate yield the factor $\delta^{-\left(1 / 2+\varepsilon_{2}\right)} \lambda^{\varepsilon_{1}}$. We thus finish the proof for the more general phase function. 
Chapter 5

Maximal functions associated with nonisotropic dilations of

some classes of hypersurfaces in $\mathbb{R}^{3}$ 
Chapter 5. Maximal functions associated with nonisotropic dilations of

\subsection{Statement of the main results}

The purpose of this chapter is to study the $L^{p}$-boundedness of certain maximal functions associated with nonisotropic dilations of surfaces in $\mathbb{R}^{3}$. We first recall some results proved by Greenleaf in [10]. Let $S$ be a smooth hypersurface in $\mathbb{R}^{n}$ with a smooth measure $d \mu$ compactly supported away from the boundary. Given an $n$-tuple $\left(a_{1}, a_{2}, \cdots, a_{n}\right)$ of nonnegative real numbers, consider the maximal operator $\mathcal{M}$ defined by

$$
\mathcal{M} f(x)=\sup _{t>0}\left|\int_{S} f\left(x-\delta_{t}(y)\right) d \mu(y)\right|,
$$

where $\delta_{t}$ denotes the nonisotropic dilation given by $\delta_{t}(x)=\left(t^{a_{1}} x_{1}, t^{a_{2}} x_{2} \cdots, t^{a_{n}} x_{n}\right)$. Supposing that at each point of supp $d \mu, S$ has everywhere non-vanishing Gaussian curvature and in addition $S$ is starshaped with respect to the origin, Greenleaf [10] showed that $\mathcal{M}$ is bounded on $L^{p}\left(\mathbb{R}^{n}\right)$ if $n \geq 3$ and $p>n /(n-1)$. Iosevich and Sawyer [14] observed that $\mathcal{M}$ often behaves much better than the maximal function with standard dilations due to a "rotational curvature" in the time parameter $t$. Let $\mathcal{H}$ be a hyperplane and $d(x, \mathcal{H})$ denote the distance from $x$ on $S$ to $\mathcal{H}$. They showed that in the case $a_{1}=a_{2}=\cdots=a_{n-1} \neq a_{n}$, the condition $d(x, \mathcal{H})^{-1} \in L_{\text {loc }}^{1 / p}(S)$ over all horizontal hyperplanes $\mathcal{H}$ is sufficient for the $L^{p}$-boundedness of $\mathcal{M}$ when the surface $S$ is given as the graph of a mixed homogeneous function with finite-type level sets. In 2010, Ikoromov, Kempe and Müller [11] discovered a connection between the $L^{p}$-boundedness of $\mathcal{M}$ and the height of a smooth, compact hypersurface of finite type in $\mathbb{R}^{3}$ satisfying a transversality assumption on $S$. Recently, Zimmermann [47] proved that the maximal averages over analytic hypersurfaces located at the origin in $\mathbb{R}^{3}$ generally behave more regularly than the maximal averages over hypersurfaces satisfying the transversality condition. It is worth to mention that in [47], he could not handle the case when the Newton polyhedron of the analytic function is given by $\left\{\left(t_{1}, t_{2}\right): t_{1} \geq n, t_{2} \geq 0\right\}$ or $\left\{\left(t_{1}, t_{2}\right): t_{1} \geq 0, t_{2} \geq m\right\}$ and does not have any line with slope which equals to minus one after any linear change of coordinates.

In this chapter, we establish $L^{p}$-estimates for maximal functions related to hypersurfaces $\left(x_{1}, x_{2}\right) \rightarrow\left(x_{1}, x_{2}, x_{2}^{d}\left(1+\mathcal{O}\left(x_{2}^{m}\right)\right)\right)$ with associated dilations $\delta_{t}(x)=\left(t^{a} x_{1}, t x_{2}, t^{d} x_{3}\right)$ for an arbitrary real number $a>0$. These results are stated in Theorem 5.1.2 and Theorem 5.1.4, and could not be covered by the theorems respectively developed in the above references. Moreover, by an alternative approach, we also get $L^{p}$-estimates for a large class of maximal functions in $\mathbb{R}^{3}$ proved in [10], [14], [11] and [47], see Theorem 5.1.1, Theorem 5.1.3 and Theorem 5.1.5.

Let $\Omega$ be an open neighborhood of the origin. Suppose $\Gamma$ is a hypersurface in $\mathbb{R}^{3}$ 
Chapter 5. Maximal functions associated with nonisotropic dilations of some classes of hypersurfaces in $\mathbb{R}^{3}$

which is parametrized as the graph of a smooth function $\Phi: \Omega \rightarrow \mathbb{R}$ at the origin, i.e. $\Gamma=\left\{(x, \Phi(x)), x \in \Omega \subset \mathbb{R}^{2}\right\}$.

In this chapter, our proofs always follow the following idea. First we "freeze" the first variable $x_{1}$ and apply the method of stationary phase in Lemma 2.2.2 to curves in $\left(x_{2}, x_{3}\right)$ - plane, then by Lemma 2.2 .3 , we can reduce to apply $L^{p}$-estimates for certain Fourier integral operators which appeared in Theorem 2.2.1, Theorem 2.3.3, Theorem 2.3.6 and Theorem 2.4.1.

Let $\delta_{t}(x)=\left(t^{a_{1}} x_{1}, t^{a_{2}} x_{2}, t^{a_{3}} x_{3}\right), a_{j}>0$ be a family of dilations.

First, we show $L^{p}$ estimates for maximal functions related to hypersurfaces with at least one non-vanishing principal curvature.

Theorem 5.1.1. Assume that $\Phi\left(x_{1}, x_{2}\right) \in C^{\infty}(\Omega)$ satisfies

$$
\partial_{2} \Phi(0,0)=0, \quad \partial_{2}^{2} \Phi(0,0) \neq 0
$$

and $2 a_{2} \neq a_{3}$. Then there exists a sufficiently small neighborhood of the origin $U \subset$ $\Omega$ such that for every positive smooth function $\eta \in C_{0}^{\infty}(U)$, the associated maximal function

$$
\mathcal{M} f(y):=\sup _{t>0}\left|\int_{\mathbb{R}^{2}} f\left(y-\delta_{t}\left(x_{1}, x_{2}, \Phi\left(x_{1}, x_{2}\right)\right)\right) \eta(x) d x\right|,
$$

initially defined on $C_{0}^{\infty}\left(\mathbb{R}^{3}\right)$, is bounded on $L^{p}\left(\mathbb{R}^{3}\right)$ for $p>2$.

Theorem 5.1.2. Let $\phi \in C^{\infty}(I)$, where $I$ is a bounded interval containing the origin. Define the maximal function by

$$
\mathcal{M} f(y):=\sup _{t>0}\left|\int_{\mathbb{R}^{2}} f\left(y-\delta_{t}\left(x_{1}, x_{2}, x_{2}^{2} \phi\left(x_{2}\right)\right)\right) \eta(x) d x\right|,
$$

where $\eta$ is supported in a sufficiently small neighborhood $U$ of the origin. Assume that $\phi$ satisfies (2.1), i.e.

$$
\phi(0) \neq 0 ; \phi^{(j)}(0)=0, j=1,2, \cdots, m-1 ; \phi^{(m)}(0) \neq 0(m \geq 1),
$$

and $2 a_{2}=a_{3}$. Then for $p>2$, there exists a constant $C_{p}$ such that the maximal operator satisfies the following estimate:

$$
\|\mathcal{M} f\|_{L^{p}} \leq C_{p}\|f\|_{L^{p}}, \quad f \in C_{0}^{\infty}\left(\mathbb{R}^{3}\right)
$$

Then we extend the above theorems to hypersurfaces of finite type. 
Chapter 5. Maximal functions associated with nonisotropic dilations of some classes of hypersurfaces in $\mathbb{R}^{3}$

Theorem 5.1.3. Assume that $\Phi\left(x_{1}, x_{2}\right) \in C^{\infty}(\Omega)$ satisfies $\Phi(0,0) \neq 0$ and $d a_{2} \neq a_{3}$, $d \geq 2$. Then there exists a sufficiently small neighborhood of the origin $U \subset \Omega$ such that for every positive smooth function $\eta \in C_{0}^{\infty}(U)$, the associated maximal function

$$
\mathcal{M} f(y):=\sup _{t>0}\left|\int_{\mathbb{R}^{2}} f\left(y-\delta_{t}\left(x_{1}, x_{2}, x_{2}^{d} \Phi\left(x_{1}, x_{2}\right)\right)\right) \eta(x) d x\right|,
$$

initially defined on $C_{0}^{\infty}\left(\mathbb{R}^{3}\right)$, is bounded on $L^{p}\left(\mathbb{R}^{3}\right)$ for $p>2$.

The case when $d a_{2}=a_{3}$ in Lemma 5.1.2 turns out to be much harder. For this case, we have the following result.

Theorem 5.1.4. Let $\phi \in C^{\infty}(I)$, where $I$ is a bounded interval containing the origin. Define the maximal function by

$$
\mathcal{M} f(y):=\sup _{t>0}\left|\int_{\mathbb{R}^{2}} f\left(y-\delta_{t}\left(x_{1}, x_{2}, x_{2}^{d} \phi\left(x_{2}\right)\right)\right) \eta(x) d x\right|
$$

where $\eta$ is supported in a sufficiently small neighborhood $U$ of the origin. Assume that $\phi$ satisfies (2.1), $d \geq 2$ and $d a_{2}=a_{3}$. Then for $p>2$, there exists a constant $C_{p}$ such that the maximal operator satisfies the following estimate:

$$
\|\mathcal{M} f\|_{L^{p}} \leq C_{p}\|f\|_{L^{p}}, \quad f \in C_{0}^{\infty}\left(\mathbb{R}^{3}\right)
$$

Finally, we notice that the surfaces in Lemma 5.1.3 are required to go through the origin. Now we consider the other case.

Theorem 5.1.5. Assume that $\Phi\left(x_{1}, x_{2}\right) \in C^{\infty}(\Omega)$ satisfies $\Phi(0,0) \neq 0$ and $d a_{2} \neq a_{3}$, $d \geq 2$. Then there exists a sufficiently small neighborhood of the origin $U \subset \Omega$ such that for every positive smooth function $\eta \in C_{0}^{\infty}(U)$, the associated maximal function

$$
\mathcal{M} f(y):=\sup _{t>0}\left|\int_{\mathbb{R}^{2}} f\left(y-\delta_{t}\left(x_{1}, x_{2}, 1+x_{2}^{d} \Phi\left(x_{1}, x_{2}\right)\right)\right) \eta(x) d x\right|,
$$

initially defined on $C_{0}^{\infty}\left(\mathbb{R}^{3}\right)$, is bounded on $L^{p}\left(\mathbb{R}^{3}\right)$ for $p>d$.

\subsection{Proofs for surfaces with one non-vanishing prin- cipal curvature}

\subsubsection{Maximal theorem with $2 a_{2} \neq a_{3}$}

In this section we give the proof for Theorem 5.1.1. 
We can always choose non-negative functions $\eta_{1}, \eta_{2} \in C_{0}^{\infty}(\mathbb{R})$ so that $\eta(x) \leq \eta_{1}\left(x_{1}\right) \eta_{2}\left(x_{2}\right)$. Since

$\left|\int_{\mathbb{R}^{2}} f\left(y-\delta_{t}\left(x_{1}, x_{2}, \Phi\left(x_{1}, x_{2}\right)\right)\right) \eta(x) d x\right| \leq \int_{\mathbb{R}^{2}}|f|\left(y-\delta_{t}\left(x_{1}, x_{2}, \Phi\left(x_{1}, x_{2}\right)\right)\right) \eta_{1}\left(x_{1}\right) \eta_{2}\left(x_{2}\right) d x$,

then we may assume $\eta(x)=\eta_{1}\left(x_{1}\right) \eta_{2}\left(x_{2}\right)$ and $f \geq 0, a_{1}=1$. Set $\left(y_{2}, y_{3}\right)=y^{\prime}$ and $\left(\xi_{2}, \xi_{3}\right)=\xi^{\prime}$. Denote $1+a_{2}+a_{3}$ by $Q$ and $\left(t^{a_{2}} \xi_{2}, t^{a_{3}} \xi_{3}\right)$ by $\delta_{t}^{\prime} \xi^{\prime}$.

Put

$$
A_{t} f(y):=\int_{\mathbb{R}^{2}} f\left(y-\delta_{t}\left(x_{1}, x_{2}, \Phi\left(x_{1}, x_{2}\right)\right)\right) \eta_{1}\left(x_{1}\right) \eta_{2}\left(x_{2}\right) d x .
$$

By means of the Fourier inversion formula, we can write

$$
A_{t} f(y)=\frac{1}{(2 \pi)^{3}} \int_{\mathbb{R}^{3}} e^{i \xi \cdot y} \int_{\mathbb{R}} e^{-i t \xi_{1} x_{1}} \eta_{1}\left(x_{1}\right) \widehat{d \mu_{x_{1}}}\left(\delta_{t}^{\prime} \xi^{\prime}\right) d x_{1} \hat{f}(\xi) d \xi
$$

where

$$
\widehat{d \mu_{x_{1}}}\left(\xi^{\prime}\right)=\int_{\mathbb{R}} e^{-i\left(\xi_{2} x_{2}+\xi_{3} \Phi\left(x_{1}, x_{2}\right)\right)} \eta_{2}\left(x_{2}\right) d x_{2} .
$$

Choose a non-negative function $\beta \in C_{0}^{\infty}(\mathbb{R})$ such that

$$
\text { supp } \beta \subset[1 / 2,2] \text { and } \sum_{j \in \mathbb{Z}} \beta\left(2^{-j} r\right)=1 \quad \text { for } r>0 .
$$

Put

$$
A_{t, j} f(y):=\frac{1}{(2 \pi)^{3}} \int_{\mathbb{R}^{3}} e^{i \xi \cdot y} \int_{\mathbb{R}} e^{-i t \xi_{1} x_{1}} \eta_{1}\left(x_{1}\right) \widehat{d \mu_{x_{1}}}\left(\delta_{t}^{\prime} \xi^{\prime}\right) d x_{1} \beta\left(2^{-j}\left|\delta_{t}^{\prime} \xi^{\prime}\right|\right) \hat{f}(\xi) d \xi,
$$

and $M_{j}$ is the corresponding maximal operator.

$$
\begin{aligned}
A_{t}^{0} f(y): & =A_{t} f(y)-\sum_{j=1}^{\infty} A_{t, j} f(y) \\
& =\frac{1}{(2 \pi)^{3}} \int_{\mathbb{R}^{3}} e^{i \xi \cdot y} \int_{\mathbb{R}} e^{-i t \xi_{1} x_{1}} \eta_{1}\left(x_{1}\right) \widehat{d \mu_{x_{1}}}\left(\delta_{t}^{\prime} \xi^{\prime}\right) d x_{1} \rho\left(\left|\delta_{t}^{\prime} \xi^{\prime}\right|\right) \hat{f}(\xi) d \xi
\end{aligned}
$$

where $\rho$ is supported in a neighborhood of the origin. Since

$$
\begin{aligned}
& \frac{1}{(2 \pi)^{3}} \int_{\mathbb{R}^{3}} e^{i \xi \cdot y} \int_{\mathbb{R}} e^{-i t \xi_{1} x_{1}} \eta_{1}\left(x_{1}\right) \widehat{d \mu_{x_{1}}}\left(\delta_{t}^{\prime} \xi^{\prime}\right) d x_{1} \rho\left(\left|\delta_{t}^{\prime} \xi^{\prime}\right|\right) d \xi \\
& =\frac{1}{(2 \pi)^{2}} \int_{\mathbb{R}} \eta_{1}\left(x_{1}\right) \int_{\mathbb{R}^{2}} e^{i \xi^{\prime} \cdot y^{\prime}} \widehat{d \mu_{x_{1}}}\left(\delta_{t}^{\prime} \xi^{\prime}\right) \rho\left(\left|\delta_{t}^{\prime} \xi^{\prime}\right|\right) d \xi^{\prime} \delta_{0}\left(y_{1}-t x_{1}\right) d x_{1} \\
& =\frac{1}{(2 \pi)^{2}} \frac{1}{t} \eta_{1}\left(\frac{y_{1}}{t}\right) \int_{\mathbb{R}^{2}} e^{i \xi^{\prime} \cdot y^{\prime}} \widehat{d \mu \frac{y_{1}}{t}}\left(\delta_{t}^{\prime} \xi^{\prime}\right) \rho\left(\left|\delta_{t}^{\prime} \xi^{\prime}\right|\right) d \xi^{\prime} \\
& =\frac{1}{(2 \pi)^{2}} t^{-Q} \eta_{1}\left(\frac{y_{1}}{t}\right) \int_{\mathbb{R}^{2}} e^{i \delta_{t^{-1}}^{\prime} \xi^{\prime} \cdot y^{\prime}} \widehat{d \mu \frac{y_{1}}{t}}\left(\xi^{\prime}\right) \rho\left(\left|\xi^{\prime}\right|\right) d \xi^{\prime}
\end{aligned}
$$


Chapter 5. Maximal functions associated with nonisotropic dilations of some classes of hypersurfaces in $\mathbb{R}^{3}$

then $A_{t}^{0} f(y)=t^{-Q} f * K\left(t^{-1} y_{1}, t^{-a_{2}} y_{2}, t^{-a_{3}} y_{3}\right)=f * K_{\delta_{t^{-1}}}(y)$, where

$$
K(y)=\frac{1}{(2 \pi)^{2}} \eta_{1}\left(y_{1}\right) \int_{\mathbb{R}^{2}} e^{i \xi^{\prime} \cdot y^{\prime}} \widehat{d \mu_{y_{1}}}\left(\xi^{\prime}\right) \rho\left(\left|\xi^{\prime}\right|\right) d \xi^{\prime}
$$

Since $\Phi$ satisfies (5.1) and $y_{1} \in \operatorname{supp} \eta_{1}$, for $N \in \mathbb{N}$ and multi-index $\alpha$ with $|\alpha|=N$, then Theorem 2.2.2 implies that

$$
\left|D_{\xi^{\prime}}^{\alpha} \widehat{d \mu_{y_{1}}}\left(\xi^{\prime}\right)\right| \leq C_{N}^{\prime}\left(1+\left|\xi^{\prime}\right|\right)^{-1 / 2}
$$

By integration by parts in $\xi^{\prime}$, we get

$$
\begin{aligned}
|K(y)| & \leq C_{N}^{\prime \prime} \frac{1}{\left(1+\left|y_{1}\right|\right)^{N}} \frac{1}{\left(1+\left|y^{\prime}\right|\right)^{N}} \int_{\mathbb{R}^{2}}\left|D_{\xi^{\prime}}^{\alpha}\left(\widehat{d \mu_{y_{1}}}\left(\xi^{\prime}\right) \rho\left(\left|\xi^{\prime}\right|\right)\right)\right| d \xi^{\prime} \\
& \leq C_{N} \prod_{i=1}^{3} \frac{1}{\left(1+\left|y_{i}\right|\right)^{N}} .
\end{aligned}
$$

Now we choose $N$ sufficiently large, then

$$
\sup _{t>0}\left|A_{t}^{0} f(y)\right| \leq C_{N} M f(y)
$$

where $M$ is the non-isotropic Hardy-Littlewood maximal operator defined by

$$
M f(y):=\sup _{t>0} \frac{1}{|B(y, t)|} \int_{B(y, t)} f(x) d x
$$

where $B(y, t)=\left\{x:|y-x|_{\delta}<t\right\}$ and $|x|_{\delta}=\max _{i=1}^{3}\left|x_{i}\right|^{1 / a_{i}}$.

So it suffices to prove that

$$
\left\|M_{j} f\right\|_{L^{p}} \leq C_{p} 2^{-j \epsilon(p)}\|f\|_{L^{p}}, \quad j \geq 1,2<p<\infty, \text { some } \epsilon(p)>0 .
$$

Since $A_{t, j}$ is localized to frequencies $\left|\delta_{t} \xi\right| \approx 2^{j}$, we can employ Lemma 2.2.4 in a similar way as in Section 2.3 to prove that

$$
\left\|M_{j}\right\|_{L^{p} \rightarrow L^{p}} \lesssim\left\|M_{j, l o c}\right\|_{L^{p} \rightarrow L^{p}}
$$

where $M_{j, l o c} f(y):=\sup _{t \in[1,2]}\left|A_{t, j} f(y)\right|$.

Indeed, for fixed $j \geq 1$ and all $\ell \in \mathbb{Z}$, let $\triangle_{\ell}^{j}$ be Littlewood-Paley operator in $\mathbb{R}^{2}$ defined by $\widehat{\triangle_{\ell}^{j}} f(\xi)=\tilde{\beta}\left(2^{-j}\left|\delta_{2^{\ell}}^{\prime} \xi^{\prime}\right|\right) \hat{f}(\xi)$, where $\tilde{\beta} \in C_{0}^{\infty}(\mathbb{R})$ is nonnegative and satisfies $\beta\left(\left|\delta_{t}^{\prime} \xi^{\prime}\right|\right)=\beta\left(\left|\delta_{t}^{\prime} \xi^{\prime}\right|\right) \tilde{\beta}\left(\left|\xi^{\prime}\right|\right) . M_{j} f(y)$ is equal to 


$$
\begin{aligned}
& \sup _{\ell \in \mathbb{Z}} \sup _{t \in[1,2]}\left|\int_{\mathbb{R}^{3}} e^{i \xi \cdot y} \int_{\mathbb{R}} e^{-i 2^{\ell} t \xi_{1} x_{1}} \eta_{1}\left(x_{1}\right) \widehat{d \mu_{x_{1}}}\left(\delta_{2^{\ell} \ell}^{\prime} \xi^{\prime}\right) d x_{1} \beta\left(2^{-j}\left|\delta_{2^{\ell}}^{\prime} \xi^{\prime}\right|\right) \tilde{\beta}\left(2^{-j}\left|\delta_{2^{\ell}}^{\prime} \xi^{\prime}\right|\right) \hat{f}(\xi) d \xi\right| \\
& =\left(\sum_{\ell \in \mathbb{Z}} \sup _{t \in[1,2]}\left|\int_{\mathbb{R}^{3}} e^{i \xi \cdot y} \int_{\mathbb{R}} e^{-i 2^{\ell} t \xi_{1} x_{1}} \eta_{1}\left(x_{1}\right) \widehat{d \mu_{x_{1}}}\left(\delta_{2^{\ell} t}^{\prime} \xi^{\prime}\right) d x_{1} \beta\left(2^{-j}\left|\delta_{2^{\ell} t}^{\prime} \xi^{\prime}\right|\right) \widehat{\triangle_{\ell}^{j}} f(\xi) d \xi\right|^{p}\right)^{1 / p} \\
& =\left(\sum_{\ell \in \mathbb{Z}} \sup _{t \in[1,2]} \mid 2^{-\ell Q} \int_{\mathbb{R}^{3}} e^{i \xi \cdot \delta_{2^{-\ell}} y} \int_{\mathbb{R}} e^{-i t \xi_{1} x_{1}} \eta_{1}\left(x_{1}\right) \widehat{d \mu_{x_{1}}}\left(\delta_{t}^{\prime} \xi^{\prime}\right) d x_{1} \beta\left(2^{-j}\left|\delta_{t}^{\prime} \xi^{\prime}\right|\right)\right. \\
& \left.\times\left.\widehat{\triangle_{\ell}^{j} f}\left(\delta_{2^{-\ell}} \xi\right) d \xi\right|^{p}\right)^{1 / p} \\
& =\left(\sum_{\ell \in \mathbb{Z}}\left|\mathcal{M}_{j, l o c}\left(\triangle_{\ell}^{j} f \circ \delta_{2^{\ell}}\right)\left(\delta_{2^{-\ell}} y\right)\right|^{p}\right)^{1 / p} .
\end{aligned}
$$

Since $p>2$, then Lemma 2.2.4 implies that

$$
\begin{aligned}
\left\|M_{j} f(y)\right\|_{L^{p}}^{p} & =\sum_{\ell \in \mathbb{Z}} \int_{\mathbb{R}^{3}}\left|\mathcal{M}_{j, l o c}\left(\triangle_{\ell}^{j} f \circ \delta_{2^{\ell}}\right)\left(\delta_{2^{-\ell}} y\right)\right|^{p} d y \\
& =\sum_{\ell \in \mathbb{Z}} 2^{\ell Q}\left\|\mathcal{M}_{j, l o c}\left(\triangle_{\ell}^{j} f \circ \delta_{2^{\ell}}\right)\right\|_{L^{p}}^{p} \\
& \leq\left\|\mathcal{M}_{j, l o c}\right\|_{L^{p} \rightarrow L^{p}}^{p} \sum_{\ell \in \mathbb{Z}} 2^{\ell Q} \int_{\mathbb{R}^{3}}\left|\triangle_{\ell}^{j} f\left(\delta_{2^{\ell}} y\right)\right|^{p} d y \\
& =\left\|\mathcal{M}_{j, l o c}\right\|_{L^{p} \rightarrow L^{p}}^{p} \sum_{\ell \in \mathbb{Z}} \int_{\mathbb{R}^{3}}\left|\triangle_{\ell}^{j} f(y)\right|^{p} d y \\
& \leq\left\|\mathcal{M}_{j, l o c}\right\|_{L^{p} \rightarrow L^{p}}^{p} \int_{\mathbb{R}} \int_{\mathbb{R}^{2}}\left(\sum_{\ell \in \mathbb{Z}}\left|\triangle_{\ell}^{j} f(y)\right|^{2}\right)^{p / 2} d y \\
& =\left\|\mathcal{M}_{j, l o c}\right\|_{L^{p} \rightarrow L^{p}}^{p}\|\|\left(\sum_{\ell \in \mathbb{Z}}\left|\triangle_{\ell}^{j} f(y)\right|^{2}\right)^{1 / 2}\left\|_{L^{p}\left(\mathbb{R}^{2}\right)}\right\|_{L^{p}(\mathbb{R})}^{p} \\
& \leq C_{p}\left\|\mathcal{M}_{j, l o c}\right\|_{L^{p} \rightarrow L^{p}}^{p}\|f\|_{L^{p}\left(\mathbb{R}^{3}\right)} .
\end{aligned}
$$

For fixed $t \in[1,2]$, let us estimate $\widehat{d \mu_{x_{1}}}\left(\delta_{t}^{\prime} \xi^{\prime}\right)$.

Set

$$
s:=s\left(\xi^{\prime}, t\right)=-\frac{t^{a_{2}} \xi_{2}}{t^{a_{3}} \xi_{3}}, \text { for } \xi_{3} \neq 0,
$$

and

$$
\Psi\left(x_{1}, x_{2}, s\right):=-s x_{2}+\Phi\left(x_{1}, x_{2}\right)
$$

We observe that

$$
\partial_{2} \Psi(0,0,0)=0 \quad \text { and } \quad \partial_{2}^{2} \Psi(0,0,0) \neq 0,
$$


Chapter 5. Maximal functions associated with nonisotropic dilations of some classes of hypersurfaces in $\mathbb{R}^{3}$

since $\Phi$ satisfies the condition (5.1). The implicit function theorem implies that there must be a smooth solution $x_{2}=\psi\left(x_{1}, s\right)$ to the equation

$$
\partial_{2} \Phi\left(x_{1}, x_{2}\right)=s
$$

where $x_{1}$ and $s$ are enough small. Here if $t \in\left[\frac{1}{2}, 4\right]$, we can choose $x_{1}$ and $s$ sufficiently small such that

$$
\partial_{2} \Phi\left(\frac{x_{1}}{t}, \psi\left(\frac{x_{1}}{t}, s\right)\right)=s .
$$

For above sufficiently small $x_{1}$, a standard application of the method of stationary phase in $x_{2}$ yields that

$$
\widehat{d \mu_{x_{1}}}\left(\delta_{t}^{\prime} \xi^{\prime}\right)=e^{-i t^{a_{3}} \xi_{3} \tilde{\Psi}\left(x_{1}, s\right)} \frac{\chi_{x_{1}}\left(t^{a_{2}} \xi_{2} / t^{a_{3}} \xi_{3}\right)}{\left(1+\left|\delta_{t}^{\prime} \xi^{\prime}\right|\right)^{1 / 2}} A_{x_{1}}\left(\delta_{t}^{\prime} \xi^{\prime}\right)+B_{x_{1}}\left(\delta_{t}^{\prime} \xi^{\prime}\right)
$$

where $\tilde{\Psi}\left(x_{1}, s\right):=\Psi\left(x_{1}, \psi\left(x_{1}, s\right), s\right)$ and $\chi_{x_{1}}$ is a smooth function supported on the set $\left\{z:|z|<\epsilon_{x_{1}}\right\}$, where $\epsilon_{x_{1}}$ can be controlled by a small positive constant independent on $x_{1}$. Moreover, $t^{a_{3}} \xi_{3} \tilde{\Psi}\left(x_{1}, s\right)$ is a smooth function which is homogeneous of degree one in $\xi^{\prime}$. Meanwhile, $A_{x_{1}}$ is a symbol of order zero such that

$$
\left|D_{\xi^{\prime}}^{\alpha} A_{x_{1}}\left(\xi^{\prime}\right)\right| \leq C_{\alpha}\left(1+\left|\xi^{\prime}\right|\right)^{-|\alpha|}
$$

where $\alpha$ is a multi-index and $C_{\alpha}$ are admissible constants. $B_{x_{1}}$ is a smooth function and satisfies

$$
\left|D_{\xi^{\prime}}^{\alpha} B_{x_{1}}\left(\xi^{\prime}\right)\right| \leq C_{\alpha, N}\left(1+\left|\xi^{\prime}\right|\right)^{-N}
$$

again with admissible constants $C_{\alpha, N}$ and $N \in \mathbb{N}$.

For fixed $t \in[1,2]$,

$$
\begin{aligned}
A_{t, j}^{0} f(y): & =\frac{1}{(2 \pi)^{3}} \int_{\mathbb{R}^{3}} e^{i \xi \cdot y} \int_{\mathbb{R}} e^{-i t \xi_{1} x_{1}} \eta_{1}\left(x_{1}\right) B_{x_{1}}\left(\delta_{t}^{\prime} \xi^{\prime}\right) d x_{1} \beta\left(2^{-j}\left|\delta_{t}^{\prime} \xi^{\prime}\right|\right) \hat{f}(\xi) d \xi \\
& =\mathcal{F}^{-1}\left\{\int_{\mathbb{R}^{3}} e^{i \xi \cdot y}\left(\int_{\mathbb{R}} e^{-i t \xi_{1} x_{1}} \eta_{1}\left(x_{1}\right) B_{x_{1}}\left(\delta_{t}^{\prime} \xi^{\prime}\right) d x_{1}\right) \beta\left(2^{-j}\left|\delta_{t}^{\prime} \xi^{\prime}\right|\right)\right\} * f(y),
\end{aligned}
$$

and

$$
\begin{aligned}
& \mathcal{F}^{-1}\left\{\int_{\mathbb{R}^{3}} e^{i \xi \cdot y}\left(\int_{\mathbb{R}} e^{-i t \xi_{1} x_{1}} \eta_{1}\left(x_{1}\right) B_{x_{1}}\left(\delta_{t}^{\prime} \xi^{\prime}\right) d x_{1}\right) \beta\left(2^{-j}\left|\delta_{t}^{\prime} \xi^{\prime}\right|\right)\right\}(y) \\
& =\frac{1}{(2 \pi)^{2}} \int_{\mathbb{R}^{2}} e^{i \xi^{\prime} \cdot y^{\prime}} \int_{\mathbb{R}} \eta_{1}\left(x_{1}\right) B_{x_{1}}\left(\delta_{t}^{\prime} \xi^{\prime}\right) \delta_{0}\left(y_{1}-t x_{1}\right) d x_{1} \beta\left(2^{-j}\left|\delta_{t}^{\prime} \xi^{\prime}\right|\right) d \xi^{\prime} \\
& =\frac{1}{(2 \pi)^{2}} t^{-Q} \eta_{1}\left(\frac{y_{1}}{t}\right) \int_{\mathbb{R}^{2}} e^{i \xi^{\prime} \cdot \delta_{t^{-1}}^{\prime} y^{\prime}} B_{y_{1} / t}\left(\xi^{\prime}\right) \beta\left(2^{-j}\left|\xi^{\prime}\right|\right) d \xi^{\prime} \\
& =\tilde{K}_{\delta_{t^{-1}}}(y)
\end{aligned}
$$


where $\tilde{K}(y)=\frac{1}{(2 \pi)^{2}} \eta_{1}\left(y_{1}\right) \int_{\mathbb{R}^{2}} e^{i \xi^{\prime} \cdot y^{\prime}} B_{y_{1}}\left(\xi^{\prime}\right) \beta\left(2^{-j}\left|\xi^{\prime}\right|\right) d \xi^{\prime}$. By (5.18) and the support of $\beta$, it is easy to get

$$
|\tilde{K}(y)| \leq C_{N} 2^{-j N} \prod_{i=1}^{3} \frac{1}{\left(1+\left|y_{i}\right|\right)^{N}}
$$

and

$$
\sup _{t \in[1,2]}\left|A_{t, j}^{0} f(y)\right| \leq C_{N} 2^{-j N} M f(y),
$$

where $M$ denotes the Hardy-Littlewood maximal operator defined by (5.10).

Put

$$
A_{t, j}^{1} f(y):=\frac{1}{(2 \pi)^{3}} \int_{\mathbb{R}^{3}} e^{i \xi \cdot y} \int_{\mathbb{R}} e^{-i t \xi_{1} x_{1}} \eta_{1}\left(x_{1}\right) e^{-i t^{a_{3}} \xi_{3} \tilde{\Psi}\left(x_{1}, s\right)} E_{x_{1}}\left(\delta_{t}^{\prime} \xi^{\prime}\right) d x_{1} \beta\left(2^{-j}\left|\delta_{t}^{\prime} \xi^{\prime}\right|\right) \hat{f}(\xi) d \xi
$$

where

$$
E_{x_{1}}\left(\delta_{t}^{\prime} \xi^{\prime}\right):=\frac{\chi_{x_{1}}\left(t^{a_{2}} \xi_{2} / t^{a_{3}} \xi_{3}\right)}{\left(1+\left|\delta_{t}^{\prime} \xi^{\prime}\right|\right)^{1 / 2}} A_{x_{1}}\left(\delta_{t}^{\prime} \xi^{\prime}\right)
$$

and denote by $\mathcal{M}_{j, l o c}^{1}$ the corresponding maximal operator. It remains to prove that

$$
\begin{aligned}
&\left\|\mathcal{M}_{j, l o c}^{1} f\right\|_{L^{p}} \leq C_{p} 2^{-j \epsilon(p)}\|f\|_{L^{p}}, \quad j \geq 1,2<p<\infty, \text { some } \epsilon(p)>0 . \\
&\left\|\mathcal{M}_{j, l o c}^{1} f\right\|_{L^{p}}= \| \sup _{t \in[1,2]} \frac{1}{(2 \pi)^{2}} \mid \int_{\mathbb{R}} \eta_{1}\left(x_{1}\right) \int_{\mathbb{R}^{2}} e^{i\left(\xi^{\prime} \cdot y^{\prime}-t^{a_{3}} \xi_{3} \tilde{\Psi}\left(x_{1}, s\right)\right)} E_{x_{1}}\left(\delta_{t}^{\prime} \xi^{\prime}\right) \\
& \times \beta\left(2^{-j}\left|\delta_{t}^{\prime} \xi^{\prime}\right|\right) f\left(y_{1}-t x_{1}, \widehat{\xi^{\prime}}\right) d \xi^{\prime} d x_{1} \mid \|_{L^{p}(d y)} \\
& \leq C \| \sup _{t \in[1,2]} \mid \int_{\mathbb{R}} \eta_{1}\left(\frac{x_{1}}{t}\right) \int_{\mathbb{R}^{2}} e^{i\left(\xi^{\prime} \cdot y^{\prime}-t^{a_{3}} \xi_{3} \tilde{\Psi}\left(\frac{x_{1}}{t}, s\right)\right)} E_{x_{1} / t}\left(\delta_{t}^{\prime} \xi^{\prime}\right) \\
& \times \beta\left(2^{-j}\left|\delta_{t}^{\prime} \xi^{\prime}\right|\right) f\left(y_{1}-x_{1}, \widehat{\xi^{\prime}}\right) d \xi^{\prime} d x_{1} \mid \|_{L^{p}(d y)} \\
&= C\left\|\sup _{t \in[1,2]}\left|\widetilde{A_{t, j}^{1}} f\right|\right\|_{L^{p}(d y)}=C\left\|\widetilde{\mathcal{M}_{j, l o c}^{1}} f\right\|_{L^{p}},
\end{aligned}
$$

where

$$
\widetilde{A_{t, j}^{1}} f(y):=\int_{\mathbb{R}} \eta_{1}\left(\frac{x_{1}}{t}\right) \int_{\mathbb{R}^{2}} e^{i\left(\xi^{\prime} \cdot y^{\prime}-t^{a_{3}} \xi_{3} \tilde{\Psi}\left(\frac{x_{1}}{t}, s\right)\right)} E_{x_{1} / t}\left(\delta_{t}^{\prime} \xi^{\prime}\right) \beta\left(2^{-j}\left|\delta_{t}^{\prime} \xi^{\prime}\right|\right) f\left(y_{1}-x_{1}, \widehat{\xi^{\prime}}\right) d \xi^{\prime} d x_{1},
$$

and

$$
\widetilde{\mathcal{M}_{j, l o c}^{1}} f(y):=\sup _{t \in[1,2]}\left|\widetilde{A_{t, j}^{1}} f(y)\right|
$$


Chapter 5. Maximal functions associated with nonisotropic dilations of some classes of hypersurfaces in $\mathbb{R}^{3}$

Set

$$
Q_{x_{1}}\left(y^{\prime}, t, \xi^{\prime}\right)=\xi^{\prime} \cdot y^{\prime}-t^{a_{3}} \xi_{3} \tilde{\Psi}\left(\frac{x_{1}}{t}, s\right) .
$$

Choose a bump function $\tilde{\rho} \in C_{0}^{\infty}(\mathbb{R})$ supported in $[1 / 2,4]$ such that $\tilde{\rho}(t)=1$ if $1 \leq t \leq$ 2. By Lemma 2.2.3, we have

$$
\begin{aligned}
\left\|\widetilde{\mathcal{M}_{j, l o c}^{1}} f(y)\right\|_{L^{p}}^{p} \leq & C_{p}\left(\int_{\mathbb{R}^{3}} \int_{1 / 2}^{4}\left|\tilde{\rho}(t) \widetilde{A_{t, j}^{1}} f(y)\right|^{p} d t d y\right)^{1 / p^{\prime}} \\
& \times\left(\int_{\mathbb{R}^{3}} \int_{1 / 2}^{4}\left|\frac{\partial}{\partial t}\left(\tilde{\rho}(t) \widetilde{A_{t, j}^{1}} f(y)\right)\right|^{p} d t d y\right)^{1 / p} .
\end{aligned}
$$

Moreover, we can choose $\tilde{\eta}_{1} \in C_{0}^{\infty}(\mathbb{R})$ such that $\tilde{\eta}_{1}=1$ on the support of $\eta_{1}$, then we have

$$
\frac{\partial}{\partial t} \widetilde{A_{t, j}^{1}} f(y)=\int_{\mathbb{R}} \tilde{\eta}_{1}\left(\frac{x_{1}}{t}\right) \int_{\mathbb{R}^{2}} e^{i Q_{x_{1}}\left(y^{\prime}, t, \xi^{\prime}\right)} h\left(t, j, x_{1}, \xi^{\prime}\right) f\left(y_{1}-x_{1}, \widehat{\xi^{\prime}}\right) d \xi^{\prime} d x_{1},
$$

where

$$
\begin{aligned}
h\left(t, j, x_{1}, \xi^{\prime}\right) & =\left(-t^{-2} x_{1} \eta_{1}^{\prime}\left(\frac{x_{1}}{t}\right)+\frac{\partial}{\partial t} Q_{x_{1}}\left(y^{\prime}, t, \xi^{\prime}\right)\right) E_{x_{1} / t}\left(\delta_{t}^{\prime} \xi^{\prime}\right) \beta\left(2^{-j}\left|\delta_{t}^{\prime} \xi^{\prime}\right|\right) \\
& +\eta_{1}\left(\frac{x_{1}}{t}\right) \frac{\partial}{\partial t} E_{x_{1} / t}\left(\delta_{t}^{\prime} \xi^{\prime}\right) \beta\left(2^{-j}\left|\delta_{t}^{\prime} \xi^{\prime}\right|\right)+\eta_{1}\left(\frac{x_{1}}{t}\right) E_{x_{1} / t}\left(\delta_{t}^{\prime} \xi^{\prime}\right) \frac{\partial}{\partial t} \beta\left(2^{-j}\left|\delta_{t}^{\prime} \xi^{\prime}\right|\right),
\end{aligned}
$$

and

$$
\left.\mid \frac{\partial}{\partial t} Q_{x_{1}}\left(y^{\prime}, t, \xi^{\prime}\right)\right)|=| \xi_{3} \frac{\partial}{\partial t}\left(t^{a_{3}} \Psi\left(\frac{x_{1}}{t}, \psi\left(\frac{x_{1}}{t}, s\right), s\right)\right)|\leq C| \xi_{3} \mid,
$$

since $t \approx 1, x_{1}$ and the support of $\chi_{x_{1}}$ are sufficiently small. Note that $A_{x_{1}}$ satisfies (5.17), then it is easy to see that $\frac{\partial}{\partial t}\left(\tilde{\rho}(t) \widetilde{A_{t, j}^{1}}\right)$ behaves like $2^{j} \widetilde{A_{t, j}^{1}}$. Clearly we only need to show the $L^{p}$-boundedness of the operator $\widetilde{A_{t, j}^{1}}$.

Furthermore, choose a function $\eta_{0} \in C_{0}^{\infty}(\mathbb{R})$, non-negative so that for arbitrary $t \in$ $[1 / 2,4], \eta_{1}\left(\frac{x_{1}}{t}\right) \leq \eta_{0}\left(x_{1}\right)$, then

$$
\begin{aligned}
& \left(\int_{\mathbb{R}^{3}} \int_{1 / 2}^{4}\left|\tilde{\rho}(t) \widetilde{A_{t, j}^{1}} f(y)\right|^{p} d t d y\right)^{1 / p} \\
& \lesssim \| \int_{\mathbb{R}} \eta_{0}\left(x_{1}\right) \mid \tilde{\rho}(t) \int_{\mathbb{R}^{2}} e^{i Q_{x_{1}}\left(y^{\prime}, t, \xi^{\prime}\right)} E_{x_{1} / t}\left(\delta_{t}^{\prime} \xi^{\prime}\right) \beta\left(2^{-j}\left|\delta_{t}^{\prime} \xi^{\prime}\right|\right) \\
& \quad \times f\left(y_{1}-x_{1}, \widehat{\xi^{\prime}}\right) d \xi^{\prime} \mid d x_{1} \|_{L^{p}\left([1 / 2,4] \times \mathbb{R}^{3}, d t d y^{\prime} d y_{1}\right)} \\
& \quad \lesssim\left\|\int_{\mathbb{R}} \eta_{0}\left(x_{1}\right)\right\| \tilde{\rho}(t) \int_{\mathbb{R}^{2}} e^{i Q_{x_{1}}\left(y^{\prime}, t, \xi^{\prime}\right)} E_{x_{1} / t}\left(\delta_{t}^{\prime} \xi^{\prime}\right) \beta\left(2^{-j}\left|\delta_{t}^{\prime} \xi^{\prime}\right|\right) \\
& \quad \times f\left(y_{1}-x_{1}, \widehat{\xi}^{\prime}\right) d \xi^{\prime}\left\|_{L^{p}\left([1 / 2,4] \times \mathbb{R}^{2}, d t d y^{\prime}\right)} d x_{1}\right\|_{L^{p}\left(\mathbb{R}, d y_{1}\right)} .
\end{aligned}
$$


Now for the inner norm we would apply the local smoothing estimate from Theorem 2.2.1, so we should verify that $Q_{x_{1}}\left(y^{\prime}, t, \xi^{\prime}\right)$ satisfies the non-degeneracy condition (2.9) and the cone condition (2.10). Apparently, the non-degeneracy condition will follow from

$$
\operatorname{rank} \partial_{\left(y^{\prime}, t\right), \xi^{\prime}}^{2} Q_{x_{1}}\left(y^{\prime}, t, \xi^{\prime}\right)=\operatorname{rank}\left(\begin{array}{cc}
1 & 0 \\
0 & 1 \\
* & *
\end{array}\right)=2 .
$$

Since $\partial_{2}^{2} \Phi(0,0) \neq 0$, we can choose $U$ sufficiently small such that

$$
C_{1}\left|\partial_{2}^{2} \Phi(0,0)\right| \geq\left|\partial_{2}^{2} \Phi\left(x_{1}, x_{2}\right)\right| \geq C_{2}\left|\partial_{2}^{2} \Phi(0,0)\right| .
$$

Also since

$$
\partial_{2} \Phi\left(\frac{x_{1}}{t}, \psi\left(\frac{x_{1}}{t}, s\right)\right)=s,
$$

by applying $\frac{\partial}{\partial s}$ on both sides, then we have

$$
\partial_{2}^{2} \Phi\left(\frac{x_{1}}{t}, \psi\left(\frac{x_{1}}{t}, s\right)\right) \partial_{2} \psi\left(\frac{x_{1}}{t}, s\right)=1,
$$

which implies that

$$
\partial_{2} \psi\left(\frac{x_{1}}{t}, s\right)=\frac{1}{\partial_{2}^{2} \Phi\left(\frac{x_{1}}{t}, \psi\left(\frac{x_{1}}{t}, s\right)\right)} .
$$

Next, we will use the fact that $\left|\xi_{2}\right| \ll\left|\xi_{3}\right| \approx\left|\xi^{\prime}\right| \approx 2^{j}(j \geq 1)$.

By the above arguments, we get

$$
\begin{aligned}
\partial_{t} Q_{x_{1}}\left(y_{0}^{\prime}, t_{0}, \xi^{\prime}\right)= & \xi_{3}\left[a_{2} t_{0}^{a_{2}-1} \frac{\xi_{2}}{\xi_{3}} \psi\left(\frac{x_{1}}{t_{0}}, s\right)+t_{0}{ }^{a_{2}} \frac{\xi_{2}}{\xi_{3}} \partial_{2} \psi\left(\frac{x_{1}}{t_{0}}, s\right)\left(a_{2}-a_{3}\right) t_{0}{ }^{a_{2}-a_{3}-1} \frac{\xi_{2}}{\xi_{3}}\right. \\
& +a_{3} t_{0}{ }^{a_{3}-1} \Phi\left(\frac{x_{1}}{t_{0}}, \psi\left(\frac{x_{1}}{t_{0}}, s\right)\right)+t_{0}{ }^{a_{3}} \partial_{2} \Phi\left(\frac{x_{1}}{t_{0}}, \psi\left(\frac{x_{1}}{t_{0}}, s\right)\right) \partial_{2} \psi\left(\frac{x_{1}}{t_{0}}, s\right) \\
& \left.\times\left(a_{2}-a_{3}\right) t_{0}{ }^{a_{2}-a_{3}-1} \frac{\xi_{2}}{\xi_{3}}+x_{1} R^{1}\left(x_{1}, t_{0}, s\right)\right] \\
= & \xi_{3}\left[a_{2} t_{0}^{a_{2}-1} \frac{\xi_{2}}{\xi_{3}} \psi\left(\frac{x_{1}}{t_{0}}, s\right)+a_{3} t_{0}{ }^{a_{3}-1} \Phi\left(\frac{x_{1}}{t_{0}}, \psi\left(\frac{x_{1}}{t_{0}}, s\right)\right)+x_{1} R^{1}\left(x_{1}, t_{0}, s\right)\right] .
\end{aligned}
$$

Furthermore, we will prove that

$$
\left|\partial_{\xi_{2}}^{2}\left(\partial_{t} Q_{x_{1}}\left(y_{0}^{\prime}, t_{0}, \xi^{\prime}\right)\right)\right| \approx 1
$$

Note that $\partial_{t} Q_{x_{1}}\left(y_{0}^{\prime}, t_{0}, \xi^{\prime}\right)$ is homogeneous of degree one in $\xi^{\prime}$, in addition to (5.28), then we obtain that

$$
\operatorname{rank}\left(\frac{\partial^{2}}{\partial \xi_{i} \partial \xi_{j}}\right)\left\langle\partial_{\left(y^{\prime}, t\right)} Q_{x_{1}}\left(y_{0}^{\prime}, t_{0}, \xi^{\prime}\right), \theta\right\rangle=1, \quad i, j=2,3,
$$


Chapter 5. Maximal functions associated with nonisotropic dilations of some classes of hypersurfaces in $\mathbb{R}^{3}$

where $\pm \theta$ are the unique directions for which $\nabla_{\xi^{\prime}}\left\langle\partial_{\left(y^{\prime}, t\right)} Q_{x_{1}}\left(y_{0}^{\prime}, t_{0}, \xi^{\prime}\right), \theta\right\rangle=0$. This implies the cone condition (2.10).

Now, let us turn to prove (5.28). Since $x_{1}$ is sufficiently small, $\left|\left(\frac{\partial}{\partial \xi_{2}}\right)^{\alpha} R^{1}\left(x_{1}, t_{0}, s\right)\right|$, $\alpha \leq 2$ is a remainder term. So we only consider

$$
\begin{gathered}
\quad\left|\frac{\partial^{2}}{\partial \xi_{2}^{2}}\left(a_{2} t_{0}^{a_{2}-1} \frac{\xi_{2}}{\xi_{3}} \psi\left(\frac{x_{1}}{t_{0}}, s\right)+a_{3} t_{0}{ }^{a_{3}-1} \Phi\left(\frac{x_{1}}{t_{0}}, \psi\left(\frac{x_{1}}{t_{0}}, s\right)\right)\right)\right| \approx 1 . \\
\frac{\partial}{\partial \xi_{2}}\left(a_{2} t_{0}^{a_{2}-1} \frac{\xi_{2}}{\xi_{3}} \psi\left(\frac{x_{1}}{t_{0}}, s\right)+a_{3} t_{0}{ }^{a_{3}-1} \Phi\left(\frac{x_{1}}{t_{0}}, \psi\left(\frac{x_{1}}{t_{0}}, s\right)\right)\right) \\
=a_{2} t_{0}^{a_{2}-1} \frac{1}{\xi_{3}} \psi\left(\frac{x_{1}}{t_{0}}, s\right)-a_{2} t_{0}^{a_{2}-1} \frac{\xi_{2}}{\xi_{3}} \partial_{2} \psi\left(\frac{x_{1}}{t_{0}}, s\right) t_{0}{ }^{a_{2}-a_{3}} \frac{1}{\xi_{3}} \\
\quad-a_{3} t_{0}{ }^{a_{3}-1} \partial_{2} \Phi\left(\frac{x_{1}}{t_{0}}, \psi\left(\frac{x}{t_{0}}, s\right)\right) \partial_{2} \psi\left(\frac{x}{t_{0}}, s\right) t_{0}{ }^{a_{2}-a_{3}} \frac{1}{\xi_{3}}+x_{1} R^{2}\left(x_{1}, t_{0}, s\right) \\
=t_{0}^{a_{2}-1} \frac{1}{\xi_{3}}\left(a_{2} \psi\left(\frac{x_{1}}{t_{0}}, s\right)+\left(a_{3}-a_{2}\right) t_{0}^{a_{2}-a_{3}} \frac{\xi_{2}}{\xi_{3}} \partial_{2} \psi\left(\frac{x_{1}}{t_{0}}, s\right)+x_{1} R^{2}\left(x_{1}, t_{0}, s\right)\right) .
\end{gathered}
$$

By the same reason as before, we only estimate

$$
\begin{aligned}
& \frac{\partial^{2}}{\partial \xi_{2}^{2}}\left(a_{2} \psi\left(\frac{x_{1}}{t_{0}}, s\right)+\left(a_{3}-a_{2}\right) t_{0}^{a_{2}-a_{3}} \frac{\xi_{2}}{\xi_{3}} \partial_{2} \psi\left(\frac{x_{1}}{t_{0}}, s\right)\right) \\
& =-a_{2} \partial_{2} \psi\left(\frac{x_{1}}{t_{0}}, s\right) t_{0}{ }^{a_{2}-a_{3}} \frac{1}{\xi_{3}}+\left(a_{3}-a_{2}\right) t_{0}^{a_{2}-a_{3}} \frac{1}{\xi_{3}} \partial_{2} \psi\left(\frac{x_{1}}{t_{0}}, s\right)-\left(a_{3}-a_{2}\right) t_{0}^{a_{2}-a_{3}} \frac{\xi_{2}}{\xi_{3}} \\
& \quad \times \partial_{2}^{2} \psi\left(\frac{x_{1}}{t_{0}}, s\right) t_{0}^{a_{2}-a_{3}} \frac{1}{\xi_{3}} \\
& =t_{0}{ }^{a_{2}-a_{3}} \frac{1}{\xi_{3}}\left(\left(a_{3}-2 a_{2}\right) \partial_{2} \psi\left(\frac{x_{1}}{t_{0}}, s\right)+\left(a_{2}-a_{3}\right) t_{0}^{a_{2}-a_{3}} \frac{\xi_{2}}{\xi_{3}} \partial_{2}^{2} \psi\left(\frac{x_{1}}{t_{0}}, s\right)\right) .
\end{aligned}
$$

(5.27) and (5.25) imply that $\partial_{2} \psi\left(\frac{x_{1}}{t_{0}}, s\right) \geq \frac{1}{C_{1}\left|\Phi^{2}(0,0)\right|} \neq 0$. Moreover, since $2 a_{2} \neq a_{3}$ and $\left|\xi_{2}\right| \ll\left|\xi_{3}\right|$, then $\left|\left(a_{3}-2 a_{2}\right) \partial_{2} \psi\left(\frac{x_{1}}{t_{0}}, s\right)\right| \approx 1$ and $\left|\left(a_{2}-a_{3}\right) t_{0}^{a_{2}-a_{3}} \frac{\xi_{2}}{\xi_{3}} \partial_{2}^{2} \psi\left(\frac{x_{1}}{t_{0}}, s\right)\right| \ll 1$. We finish the proof of (5.30) and (5.28).

Hence we can apply Theorem 2.2.1 for $\mu=-1 / 2$, and obtain

$$
\begin{aligned}
& \left(\int_{\mathbb{R}^{3}} \int_{1 / 2}^{4}\left|\tilde{\rho}(t) \widetilde{A_{t, j}^{1}} f(y)\right|^{p} d t d y\right)^{1 / p} \\
& \leq C_{p} 2^{-j(1 / p+\epsilon(p))}\left\|\int_{\mathbb{R}} \eta_{0}\left(x_{1}\right)\right\| f\left(y_{1}-x_{1}, y^{\prime}\right)\left\|_{L^{p}\left(\mathbb{R}^{2}, d y^{\prime}\right)} d x_{1}\right\|_{L^{p}\left(\mathbb{R}, d y_{1}\right)} \\
& \leq C_{p} 2^{-j(1 / p+\epsilon(p))}\|f\|_{L^{p}}\left\|\eta_{0}\right\|_{L^{1}},
\end{aligned}
$$


and thus by (5.24), we get

$$
\begin{aligned}
& \left\|\widetilde{\mathcal{M}_{j, l o c}^{1}} f(y)\right\|_{L^{p}}^{p} \\
& \leq C_{p}\left(2^{-j(1 / p+\epsilon(p))}\|f\|_{L^{p}}\left\|\eta_{0}\right\|_{L^{1}}\right)^{p-1}\left(2^{-j(-1+1 / p+\epsilon(p))}\|f\|_{L^{p}}\left\|\eta_{0}\right\|_{L^{1}}\right) \\
& =C_{p} 2^{-j p \epsilon(p)}\|f\|_{L^{p}}^{p}\left\|\eta_{0}\right\|_{L^{1}}^{p} .
\end{aligned}
$$

Now we have finished the proof of Theorem 5.1.1.

\subsubsection{Maximal theorem with $2 a_{2}=a_{3}$}

In this section, we will prove Theorem 5.1.2.

First we may assume $\eta(x)=\eta_{1}\left(x_{1}\right) \eta_{2}\left(x_{2}\right)$ with non-negative functions $\eta_{1}, \eta_{2} \in C_{0}^{\infty}(\mathbb{R})$, and $f \geq 0, a_{2}=1, a_{3}=2$. Let $\left(y_{2}, y_{3}\right)=y^{\prime}$ and $\left(\xi_{2}, \xi_{3}\right)=\xi^{\prime}$. Denote $a_{1}+3$ by $Q$ and $\left(t \xi_{2}, t^{2} \xi_{3}\right)$ by $\tilde{\delta}_{t} \xi^{\prime}$. We choose $B>0$ very small and $\tilde{\rho} \in C_{0}^{\infty}(\mathbb{R})$ such that supp $\tilde{\rho} \subset\{x \in \mathbb{R}: B / 2 \leq|x| \leq 2 B\}$ and $\sum_{k} \tilde{\rho}\left(2^{k} x\right)=1$.

Put

$$
A_{t} f(y):=\int_{\mathbb{R}^{2}} f\left(y_{1}-t^{a_{1}} x_{1}, y_{2}-t x_{2}, y_{3}-t^{2} x_{2}^{2} \phi\left(x_{2}\right)\right) \eta(x) d x=\sum_{k} \widetilde{A_{t}^{k}} f(y)
$$

where $\widetilde{A_{t}^{k}} f(y)=\int_{\mathbb{R}^{2}} f\left(y_{1}-t^{a_{1}} x_{1}, y_{2}-t x_{2}, y_{3}-t^{2} x_{2}^{2} \phi\left(x_{2}\right)\right) \eta_{1}\left(x_{1}\right) \eta_{2}\left(x_{2}\right) \tilde{\rho}\left(2^{k} x_{2}\right) d x$.

Define the isometric operator $\mathrm{T}$ on $L^{p}\left(\mathbb{R}^{3}\right)$ by

$$
T f\left(x_{1}, x_{2}, x_{3}\right)=2^{3 k / p} f\left(x_{1}, 2^{k} x_{2}, 2^{2 k} x_{3}\right) .
$$

One can easily compute that

$$
T^{-1} \widetilde{A_{t}^{k}} T f(y)=2^{-k} \int_{\mathbb{R}^{2}} f\left(y_{1}-t^{a_{1}} x_{1}, y_{2}-t x_{2}, y_{3}-t^{2} x_{2}^{2} \phi\left(\frac{x_{2}}{2^{k}}\right)\right) \eta_{1}\left(x_{1}\right) \eta_{2}\left(\frac{x_{2}}{2^{k}}\right) \rho\left(x_{2}\right) d x .
$$

It suffices to prove the following estimate

$$
\sum_{k} 2^{-k}\left\|\sup _{t>0}\left|A_{t}^{k}\right|\right\|_{L^{p} \rightarrow L^{p}} \leq C_{p}, \quad p>2
$$

where

$$
A_{t}^{k} f(y):=\int_{\mathbb{R}^{2}} f\left(y_{1}-t^{a_{1}} x_{1}, y_{2}-t x_{2}, y_{3}-t^{2} x_{2}^{2} \phi\left(\frac{x_{2}}{2^{k}}\right)\right) \eta_{1}\left(x_{1}\right) \eta_{2}\left(\frac{x_{2}}{2^{k}}\right) \rho\left(x_{2}\right) d x
$$



some classes of hypersurfaces in $\mathbb{R}^{3}$

By means of the Fourier inversion formula, we can write

$$
A_{t}^{k} f(y)=\frac{1}{(2 \pi)^{3}} \int_{\mathbb{R}^{3}} e^{i \xi \cdot y} \widehat{\eta}_{1}\left(t^{a_{1}} \xi_{1}\right) \widehat{d \mu_{k, m}}\left(\tilde{\delta}_{t} \xi^{\prime}\right) \hat{f}(\xi) d \xi
$$

where

$$
\widehat{d \mu_{k, m}}\left(\tilde{\delta}_{t} \xi^{\prime}\right)=\int_{\mathbb{R}} e^{-i\left(t \xi_{2} x_{2}+t^{2} \xi_{3} x_{2}^{2} \phi\left(\frac{x_{2}}{2^{k}}\right)\right)} \tilde{\rho}\left(x_{2}\right) \eta_{2}\left(2^{-k} x_{2}\right) d x_{2}
$$

Choose a non-negative function $\beta \in C_{0}^{\infty}(\mathbb{R})$ such that

$$
\text { supp } \beta \subset[1 / 2,2] \text { and } \sum_{j \in \mathbb{Z}} \beta\left(2^{-j} r\right)=1 \quad \text { for } \quad r>0 .
$$

and set

$$
A_{t, j}^{k} f(y):=\frac{1}{(2 \pi)^{3}} \int_{\mathbb{R}^{3}} e^{i \xi \cdot y} \widehat{\eta}_{1}\left(t^{a_{1}} \xi_{1}\right) \widehat{d \mu_{k, m}}\left(\tilde{\delta}_{t} \xi^{\prime}\right) \beta\left(2^{-j}\left|\tilde{\delta}_{t} \xi^{\prime}\right|\right) \hat{f}(\xi) d \xi
$$

and denote by $M_{j}^{k}$ the corresponding maximal operator.

From the proof of inequality (5.9), it is easy to see that the supremum of the absolute value of the difference between $A_{t}^{k} f(y)$ and $\sum_{j=1}^{\infty} A_{t, j}^{k} f(y)$ is dominated by the HardyLittlewood maximal function $M f(y)$ defined by (5.10). It remains to consider the $L^{p}$-boundedness $(2<p<\infty)$ of the maximal operator $M_{j}^{k}$ for $j \geq 1$.

Since $A_{t, j}^{k}$ is localized to frequencies $\left|\tilde{\delta}_{t} \xi^{\prime}\right| \approx 2^{j}$, combining the method of Section 5.2.1 and Lemma 2.2.4, then we will have

$$
\left\|M_{j}^{k}\right\|_{L^{p} \rightarrow L^{p}} \lesssim\left\|M_{j, l o c}^{k}\right\|_{L^{p} \rightarrow L^{p}}
$$

where $M_{j, l o c}^{k} f(y):=\sup _{t \in[1,2]}\left|A_{t, j}^{k} f(y)\right|$.

For fixed $t \in[1,2]$, let us estimate $\widehat{d \mu_{k, m}}\left(\tilde{\delta}_{t} \xi^{\prime}\right)$.

Set

$$
\begin{gathered}
\delta:=2^{-k}, \quad s:=s\left(\xi^{\prime}, t\right)=-\frac{\xi_{2}}{t \xi_{3}}, \text { for } \xi_{3} \neq 0, \\
\Phi\left(s, x_{2}, \delta\right):=-s x_{2}+x_{2}^{2} \phi\left(\delta x_{2}\right) .
\end{gathered}
$$

A similar argument as in Section 5.2.1 and Section 2.3.2 shows that we can reduce to considering for $j \geq 1$, the $L^{p}$-boundedness of the operator $\widetilde{A_{t, j}^{k}}$ given by

$$
\begin{aligned}
\widetilde{A_{t, j}^{k}} f(y):= & \int_{\mathbb{R}} \eta_{1}\left(\frac{x_{1}}{t^{a_{1}}}\right) \int_{\mathbb{R}^{2}} e^{i\left(\xi^{\prime} \cdot y^{\prime}-t^{2} \xi_{3} \tilde{\Phi}(s, \delta)\right)} \chi_{k, m}\left(\frac{\xi_{2}}{t \xi_{3}}\right) \frac{A_{k, m}\left(\tilde{\delta}_{t} \xi^{\prime}\right)}{\left(1+\left|\tilde{\delta}_{t} \xi^{\prime}\right|\right)^{1 / 2}} \beta\left(2^{-j}\left|\tilde{\delta}_{t} \xi^{\prime}\right|\right) \\
& \times f\left(y_{1}-x_{1}, \widehat{\xi}^{\prime}\right) d \xi^{\prime} d x_{1}
\end{aligned}
$$


Chapter 5. Maximal functions associated with nonisotropic dilations of some classes of hypersurfaces in $\mathbb{R}^{3}$

where $\tilde{\Phi}(s, \delta):=\Phi(s, \tilde{q}(s, \delta), \delta)$ and $x_{2}=\tilde{q}(s, \delta)$ is the solution of the equation $\partial_{2} \Phi\left(s, x_{2}, \delta\right)=0$ and smoothly converges to the solution $\tilde{q}(s, 0)=s$ of the equation $\partial_{2} \Phi(s, x, 0)=0$ if we assume $\phi(0)=1 / 2$. The phase function can be written as

$$
-t^{2} \xi_{3} \tilde{\Phi}(s, \delta):=\frac{\xi_{2}^{2}}{2 \xi_{3}}+(-1)^{m+1} \delta^{m} \frac{\phi^{(m)}(0)}{m !} \frac{\xi_{2}^{m+2}}{t^{m} \xi_{3}^{m+1}}+R\left(t, \xi^{\prime}, \delta\right),
$$

which is homogeneous of degree one and can be considered as a small perturbation of $\frac{\xi_{2}^{2}}{2 \xi_{3}}+(-1)^{m+1} \delta^{m} \frac{\phi^{(m)(0)}}{m !} \frac{\xi_{2}^{m+2}}{t^{m} \xi_{3}^{m+1}} . \chi_{k, m}$ is a smooth function supported in the interval $\left[c_{k, m}, \tilde{c}_{k, m}\right]$, for certain non-zero constants $c_{k, m}$ and $\tilde{c}_{k, m}$ dependent only on $k$ and $m$. $A_{k, m}$ is a symbol of order zero and $\left\{A_{k, m}\left(\tilde{\delta}_{t} \xi^{\prime}\right)\right\}_{k}$ is contained in a bounded subset of symbols of order zero. Denote by $\widehat{\mathcal{M}_{j, l o c}^{k}}$ the corresponding maximal operator.

Following the argument of $(2.46)$, we can choose a bump function $\rho_{1} \in C_{0}^{\infty}\left(\mathbb{R}^{2} \times\right.$ $[1 / 2,4])$, by Lemma 2.2 .3 , we have

$$
\begin{aligned}
\left\|\widetilde{\mathcal{M}_{j, l o c}^{k}} f\right\|_{L^{p}}^{p} \leq & C_{p}\left(\int_{\mathbb{R}^{3}} \int_{1 / 2}^{4}\left|\rho_{1}\left(y^{\prime}, t\right) \widetilde{A_{t, j}^{k}} f(y)\right|^{p} d t d y\right)^{1 / p^{\prime}} \\
& \times\left(\int_{\mathbb{R}^{3}} \int_{1 / 2}^{4}\left|\frac{\partial}{\partial t}\left(\rho_{1}\left(y^{\prime}, t\right) \widetilde{A_{t, j}^{k}} f(y)\right)\right|^{p} d t d y\right)^{1 / p} .
\end{aligned}
$$

Moreover, we choose a non-negative function $\tilde{\eta}_{1} \in C_{0}^{\infty}(\mathbb{R})$ such that $\eta_{1}=1$ on the support of $\eta_{1}$, then

$$
\frac{\partial}{\partial t}\left(\widetilde{A_{t, j}^{k}} f(y)\right)=\int_{\mathbb{R}} \tilde{\eta}_{1}\left(\frac{x_{1}}{t}\right) \int_{\mathbb{R}^{2}} e^{i\left(\xi^{\prime} \cdot y^{\prime}-t^{2} \xi_{3} \tilde{\Phi}(s, \delta)\right)} h_{k, m}\left(y, t, j, \xi^{\prime}\right) f\left(y_{1}-x_{1}, \widehat{\xi}^{\prime}\right) d \xi^{\prime} d x_{1},
$$

where

$$
\begin{aligned}
h_{k, m}\left(y, t, j, \xi^{\prime}\right)= & \left(\frac{\partial}{\partial t} \rho_{1}\left(y^{\prime}, t\right) \eta_{1}\left(\frac{x_{1}}{t}\right)-\rho_{1}\left(y^{\prime}, t\right) \frac{x_{1}}{t^{2}} \eta_{1}^{\prime}\left(\frac{x_{1}}{t}\right)-\rho_{1}\left(y^{\prime}, t\right) \eta_{1}\left(\frac{x_{1}}{t}\right) \frac{\partial}{\partial t}\left(t^{2} \xi_{3} \tilde{\Phi}(s, \delta)\right)\right) \\
& \times \chi_{k, m}\left(\frac{\xi_{2}}{t \xi_{3}}\right) \frac{A_{k, m}\left(\tilde{\delta}_{t} \xi^{\prime}\right)}{\left(1+\left|\tilde{\delta}_{t} \xi^{\prime}\right|\right)^{1 / 2}} \beta\left(2^{-j}\left|\tilde{\delta}_{t} \xi^{\prime}\right|\right) \\
& -\rho_{1}\left(y^{\prime}, t\right) \eta_{1}\left(\frac{x_{1}}{t}\right) \frac{\xi_{2}}{t^{2} \xi_{3}} \chi_{k, m}^{\prime}\left(\frac{\xi_{2}}{t \xi_{3}}\right) \frac{A_{k, m}\left(\tilde{\delta}_{t} \xi^{\prime}\right)}{\left(1+\left|\tilde{\delta}_{t} \xi^{\prime}\right|\right)^{1 / 2}} \beta\left(2^{-j}\left|\tilde{\delta}_{t} \xi^{\prime}\right|\right) \\
& +\rho_{1}\left(y^{\prime}, t\right) \eta_{1}\left(\frac{x_{1}}{t}\right) \chi_{k, m}\left(\frac{\xi_{2}}{t \xi_{3}}\right) \frac{\partial}{\partial t}\left(\frac{A_{k, m}\left(\tilde{\delta}_{t} \xi^{\prime}\right)}{\left(1+\left|\tilde{\delta}_{t} \xi^{\prime}\right|\right)^{1 / 2}}\right) \beta\left(2^{-j}\left|\tilde{\delta}_{t} \xi^{\prime}\right|\right) \\
& +\rho_{1}\left(y^{\prime}, t\right) \eta_{1}\left(\frac{x_{1}}{t}\right) \chi_{k, m}\left(\frac{\xi_{2}}{t \xi_{3}}\right) \frac{A_{k, m}\left(\tilde{\delta}_{t} \xi^{\prime}\right)}{\left(1+\left|\tilde{\delta}_{t} \xi^{\prime}\right|\right)^{1 / 2}} \frac{\partial}{\partial t}\left(\beta\left(2^{-j}\left|\tilde{\delta}_{t} \xi^{\prime}\right|\right)\right) .
\end{aligned}
$$

Since $t \approx 1, x_{1}$ and support of $\chi_{k, m}$ are sufficiently small, by (5.35), then $\left|t^{2} \xi_{3} \tilde{\Phi}(s, \delta)\right| \approx$ $2^{j} \delta^{m}$. Moreover, $\left\{A_{k, m}\left(\tilde{\delta}_{t} \xi^{\prime}\right)\right\}_{k}$ is contained in a bounded subset of symbols of order 
Chapter 5. Maximal functions associated with nonisotropic dilations of some classes of hypersurfaces in $\mathbb{R}^{3}$

zero, so we obtain

$$
\left|h_{k, m}\left(y, t, j, \xi^{\prime}\right)\right| \lesssim 2^{j / 2} \delta^{m}+2^{-j / 2}
$$

Now it is easy to see that $\left(2^{j / 2} \delta^{m}+2^{-j / 2}\right)^{-1} \frac{\partial}{\partial t}\left(\rho_{1}\left(y^{\prime}, t\right) \widetilde{A_{t, j}^{k}} f\right)$ behaves like $2^{j / 2} \widetilde{A_{t, j}^{k}} f$. It is sufficient to estimate $\left\|\rho_{1}\left(y^{\prime}, t\right) \widetilde{A_{t, j}^{k}} f(y)\right\|_{L^{p}\left(\mathbb{R}^{3} \times[1 / 2,4], d t d y\right)}$.

Furthermore, choosing a function $\eta_{0} \in C_{0}^{\infty}(\mathbb{R})$, non-negative, such that for arbitrary $t \in[1,2], a_{1}>0, \eta_{1}\left(\frac{x_{1}}{t^{a_{1}}}\right) \leq \eta_{0}\left(x_{1}\right)$, we get

$$
\begin{aligned}
& \left|\int_{\mathbb{R}} \eta_{1}\left(\frac{x_{1}}{t^{a_{1}}}\right) \int_{\mathbb{R}^{2}} e^{i\left(\xi^{\prime} \cdot y^{\prime}-t^{2} \xi_{3} \tilde{\Phi}(s, \delta)\right)} \chi_{k, m}\left(\frac{\xi_{2}}{t \xi_{3}}\right) \frac{A_{k, m}\left(\tilde{\delta}_{t} \xi^{\prime}\right)}{\left(1+\left|\tilde{\delta}_{t} \xi^{\prime}\right|\right)^{1 / 2}} \beta\left(2^{-j}\left|\tilde{\delta}_{t} \xi^{\prime}\right|\right) f\left(y_{1}-x_{1}, \widehat{\xi}^{\prime}\right) d \xi^{\prime} d x_{1}\right| \\
& \leq \int_{\mathbb{R}} \eta_{0}\left(x_{1}\right)\left|\int_{\mathbb{R}^{2}} e^{i\left(\xi^{\prime} \cdot y^{\prime}-t^{2} \xi_{3} \tilde{\Phi}(s, \delta)\right)} \chi_{k, m}\left(\frac{\xi_{2}}{t \xi_{3}}\right) \frac{A_{k, m}\left(\tilde{\delta}_{t} \xi^{\prime}\right)}{\left(1+\left|\tilde{\delta}_{t} \xi^{\prime}\right|\right)^{1 / 2}} \beta\left(2^{-j}\left|\tilde{\delta}_{t} \xi^{\prime}\right|\right) f\left(y_{1}-x_{1}, \widehat{\xi}^{\prime}\right) d \xi^{\prime}\right| d x_{1} .
\end{aligned}
$$

In order to apply the regularity estimate Lemma 2.3 .5 for $j \leq 9 k m$ and the local smoothing estimate Theorem 2.3.6 for $j>9 \mathrm{~km}$ of the Fourier integral operators not satisfying the "cinematic curvature condition" uniformly, we freeze $x_{1}$, in fact, by Minkowski's and Young's inequalities, we have

$$
\begin{aligned}
& \left\|\rho_{1}\left(y^{\prime}, t\right) \widetilde{A_{t, j}^{k}} f(y)\right\|_{L^{p}\left(\mathbb{R}^{3} \times[1 / 2,4], d t d y\right)} \\
& \leq C\left(\int _ { \mathbb { R } ^ { 3 } } \int \left(\rho_{1}\left(y^{\prime}, t\right) \int_{\mathbb{R}} \eta_{0}\left(x_{1}\right) \mid \int_{\mathbb{R}^{2}} e^{i\left(\xi^{\prime} \cdot y^{\prime}-t^{2} \xi_{3} \tilde{\Phi}(s, \delta)\right)} \chi_{k, m}\left(\frac{\xi_{2}}{t \xi_{3}}\right) \frac{A_{k, m}\left(\tilde{\delta}_{t} \xi^{\prime}\right)}{\left(1+\left|\tilde{\delta}_{t} \xi^{\prime}\right|\right)^{1 / 2}} \beta\left(2^{-j}\left|\tilde{\delta}_{t} \xi^{\prime}\right|\right)\right.\right. \\
& \left.\left.\quad \times f\left(y_{1}-x_{1}, \widehat{\xi^{\prime}}\right) d \xi^{\prime} \mid d x_{1}\right)^{p} d t d y\right)^{1 / p} \\
& \leq C\left\|\int_{\mathbb{R}} \eta_{0}\left(x_{1}\right)\right\| \rho_{1}\left(y^{\prime}, t\right) \int_{\mathbb{R}^{2}} e^{i\left(\xi^{\prime} \cdot y^{\prime}-t^{2} \xi_{3} \tilde{\Phi}(s, \delta)\right)} \chi_{k, m}\left(\frac{\xi_{2}}{t \xi_{3}}\right) \frac{A_{k, m}\left(\tilde{\delta}_{t} \xi^{\prime}\right)}{\left(1+\left|\tilde{\delta}_{t} \xi^{\prime}\right|\right)^{1 / 2}} \beta\left(2^{-j}\left|\tilde{\delta}_{t} \xi^{\prime}\right|\right) \\
& \quad \times f\left(y_{1}-x_{1}, \widehat{\xi}^{\prime}\right) d \xi^{\prime}\left\|_{L^{p}\left(\mathbb{R}^{2} \times\left[\frac{1}{2}, 4\right], d t d y^{\prime}\right)} d x_{1}\right\|_{L^{p}\left(\mathbb{R}, d y_{1}\right)} \\
& \leq
\end{aligned}
$$

Finally, together with the arguments from Section 2.3.2, we finish the proof. 
Chapter 5. Maximal functions associated with nonisotropic dilations of some classes of hypersurfaces in $\mathbb{R}^{3}$

\subsection{Proofs for surfaces of finite type}

\subsubsection{Maximal function theorem with $d a_{2} \neq a_{3}$}

Theorem 5.1 .3 will be proved in this section.

First we assume $\eta(x)=\eta_{1}\left(x_{1}\right) \eta_{2}\left(x_{2}\right)$ with non-negative functions $\eta_{1}, \eta_{2} \in C_{0}^{\infty}(\mathbb{R})$ and $f \geq 0, a_{1}=1$. Let $\left(y_{2}, y_{3}\right)=y^{\prime}$ and $\left(\xi_{2}, \xi_{3}\right)=\xi^{\prime}$. Denote $\left(t^{a_{2}} \xi_{2}, t^{a_{3}} \xi_{3}\right)$ by $\delta_{t}^{\prime} \xi^{\prime}$. We choose $B>0$ very small and $\tilde{\rho} \in C_{0}^{\infty}(\mathbb{R})$ such that supp $\tilde{\rho} \subset\{x \in \mathbb{R}: B / 2 \leq|x| \leq 2 B\}$ and $\sum_{k} \tilde{\rho}\left(2^{k} x\right)=1$.

Put

$$
A_{t} f(y):=\int_{\mathbb{R}^{2}} f\left(y_{1}-t x_{1}, y_{2}-t^{a_{2}} x_{2}, y_{3}-t^{a_{3}} x_{2}^{d} \Phi(x)\right) \eta(x) d x:=\sum_{k} \widetilde{A_{t}^{k}} f(y),
$$

where $\widetilde{A_{t}^{k}} f(y):=\int_{\mathbb{R}^{2}} f\left(y_{1}-t x_{1}, y_{2}-t^{a_{2}} x_{2}, y_{3}-t^{a_{3}} x_{2}^{d} \Phi(x)\right) \eta(x) \tilde{\rho}\left(2^{k} x_{2}\right) d x$.

Define the isometric operator on $L^{p}\left(\mathbb{R}^{3}\right)$ by

$$
T f\left(x_{1}, x_{2}, x_{3}\right)=2^{k(d+1) / p} f\left(x_{1}, 2^{k} x_{2}, 2^{d k} x_{3}\right) .
$$

By the arguments in Section 5.2.1, it suffices to prove the following inequality

$$
\sum_{k} 2^{-k}\left\|\sup _{t>0}\left|A_{t}^{k}\right|\right\|_{L^{p} \rightarrow L^{p}} \leq C_{p}
$$

where

$$
A_{t}^{k} f(y)=\int_{\mathbb{R}^{2}} f\left(y_{1}-t x_{1}, y_{2}-t^{a_{2}} x_{2}, y_{3}-t^{a_{3}} x_{2}^{d} \Phi\left(x_{1}, \frac{x_{2}}{2^{k}}\right)\right) \eta_{1}\left(x_{1}\right) \eta_{2}\left(2^{-k} x_{2}\right) \tilde{\rho}\left(x_{2}\right) d x .
$$

By means of the Fourier inversion formula, we can write

$$
A_{t}^{k} f(y)=\frac{1}{(2 \pi)^{3}} \int_{\mathbb{R}^{3}} e^{i \xi \cdot y} \int_{\mathbb{R}} e^{-i t \xi_{1} x_{1}} \eta_{1}\left(x_{1}\right) \widehat{d \mu_{k, x_{1}, d}}\left(\delta_{t}^{\prime} \xi^{\prime}\right) d x_{1} \hat{f}(\xi) d \xi
$$

where

$$
\widehat{d \mu_{k, x_{1}, d}}\left(\delta_{t}^{\prime} \xi^{\prime}\right)=\int_{\mathbb{R}} e^{-i\left(t^{a_{2}} \xi_{2} x_{2}+t^{a_{3}} \xi_{3} x_{2}^{d} \Phi\left(x_{1}, \frac{x_{2}}{2^{k}}\right)\right)} \tilde{\rho}\left(x_{2}\right) \eta_{2}\left(2^{-k} x_{2}\right) d x_{2} .
$$

Choose a non-negative function $\beta \in C_{0}^{\infty}(\mathbb{R})$ such that

$$
\operatorname{supp} \beta \subset[1 / 2,2] \text { and } \quad \sum_{j \in \mathbb{Z}} \beta\left(2^{-j} r\right)=1 \quad \text { for } \quad r>0 \text {. }
$$


Define

$$
A_{t, j}^{k} f(y):=\frac{1}{(2 \pi)^{3}} \int_{\mathbb{R}^{3}} e^{i \xi \cdot y} \int_{\mathbb{R}} e^{-i t \xi_{1} x_{1}} \eta_{1}\left(x_{1}\right) \widehat{d \mu_{k, x_{1}, d}}\left(\delta_{t}^{\prime} \xi^{\prime}\right) d x_{1} \beta\left(2^{-j}\left|\delta_{t}^{\prime} \xi^{\prime}\right|\right) \hat{f}(\xi) d \xi,
$$

and denote by $M_{j}^{k}$ the corresponding maximal operator.

From the arguments of Section 5.2.1, we see that $\sup _{t>0}\left|\sum_{j \leq 0}^{\infty} A_{t, j}^{k} f(y)\right|$ can be dominated by the Hardy-Littlewood maximal function $M f(y)$ defined by (5.10), then it suffices to prove that

$$
\left\|M_{j}^{k}\right\|_{L^{p} \rightarrow L^{p}} \leq C_{p} 2^{-j \epsilon(p)}, \quad j \geq 1,2<p<\infty, \text { some } \epsilon(p)>0 .
$$

Since $A_{t, j}^{k}$ is localized to frequencies $\left|\delta_{t}^{\prime} \xi^{\prime}\right| \approx 2^{j}$, we can still use Lemma 2.2.4 to prove that

$$
\left\|M_{j}^{k}\right\|_{L^{p} \rightarrow L^{p}} \lesssim\left\|M_{j, l o c}^{k}\right\|_{L^{p} \rightarrow L^{p}}
$$

where $M_{j, l o c}^{k} f(y):=\sup _{t \in[1,2]}\left|A_{t, j}^{k} f(y)\right|$.

For fixed $t \in[1,2]$, let us estimate $\widehat{d \mu_{k, x_{1}}, d}\left(\delta_{t}^{\prime} \xi^{\prime}\right)$.

Set

$$
\delta:=2^{-k}, \quad s:=s\left(\xi^{\prime}, t\right)=-\frac{t^{a_{2}} \xi_{2}}{t^{a_{3}} \xi_{3}}, \text { for } \xi_{3} \neq 0
$$

and

$$
\Psi\left(x_{1}, x_{2}, s, \delta\right):=-s x_{2}+\Phi_{k}\left(x_{1}, x_{2}\right),
$$

where $\Phi_{k}\left(x_{1}, x_{2}\right):=x_{2}^{d} \Phi\left(x_{1}, \delta x_{2}\right)$.

Since $x_{2} \approx 1$ here, in addition to $\Phi(0,0) \neq 0$, then we can reduce our proof to the case $d=2$. So from now on we can proceed similarly as in Section 5.2.1.

This finishes the proof of Theorem 5.1.3.

\subsubsection{Maximal theorem with $d a_{2}=a_{3}$}

Combining the proofs of Section 5.2.2 and Section 2.4, it is easy to get Theorem 5.1.4. We omit the details here.

\subsection{Proofs for surfaces not passing through the ori- gin}

We will show the proof of Theorem 5.1.5, where $d a_{2} \neq a_{3}$. 
Chapter 5. Maximal functions associated with nonisotropic dilations of some classes of hypersurfaces in $\mathbb{R}^{3}$

Following the proof of Section 5.3.1, we only modify some places.

Define

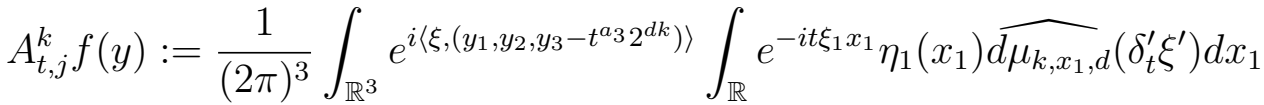

$$
\begin{aligned}
& \times \beta\left(2^{-j}\left|\delta_{t}^{\prime} \xi^{\prime}\right|\right) \hat{f}(\xi) d \xi
\end{aligned}
$$

Set $A_{t}^{k, 0} f(y):=\sum_{j \leq 0}^{\infty} A_{t, j}^{k} f(y)$ and $M^{k, 0} f(y):=\sup _{t>0}\left|A_{t}^{k, 0} f(y)\right|$. It is easy to see that

$$
A_{t}^{k, 0} f(y)=f * K_{\delta_{t^{-1}}}^{\sigma}(y)
$$

where $\sigma=\left(0,0,2^{d k}\right)$ and $K_{\delta_{t^{-1}}}^{\sigma}(y)=t^{-Q} K^{\sigma}\left(t^{-1} y_{1}, t^{-a_{2}} y_{2}, t^{-a_{3}} y_{3}\right)$ and $K^{\sigma}$ is the translate

$$
K^{\sigma}(y)=K(y-\sigma)
$$

Indeed, since

$$
\begin{aligned}
& \frac{1}{(2 \pi)^{3}} \int_{\mathbb{R}^{3}} e^{i\left\langle\xi,\left(y_{1}, y_{2}, y_{3}-t^{a_{3} 2^{d k}}\right)\right\rangle} \int_{\mathbb{R}} e^{-i t \xi_{1} x_{1}} \eta_{1}\left(x_{1}\right) \widehat{d \mu_{k, x_{1}, d}}\left(\delta_{t}^{\prime} \xi^{\prime}\right) d x_{1} \rho\left(\left|\delta_{t}^{\prime} \xi^{\prime}\right|\right) d \xi
\end{aligned}
$$

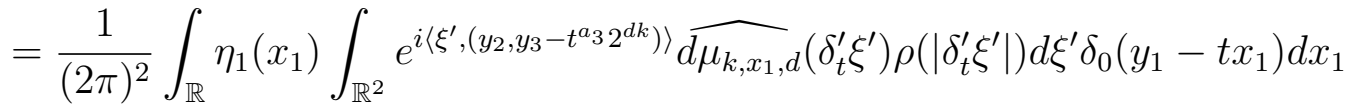

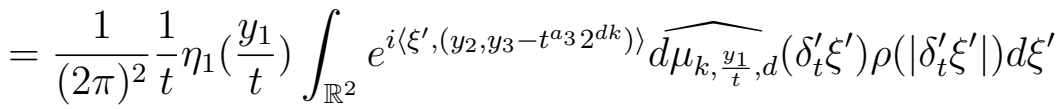

$$
\begin{aligned}
& =\frac{1}{(2 \pi)^{2}} t^{-Q} \eta_{1}\left(\frac{y_{1}}{t}\right) \int_{\mathbb{R}^{2}} e^{i\left\langle\xi^{\prime}, \delta_{t^{-1}}^{\prime} y^{\prime}-\left(0,2^{d k}\right)\right\rangle} \widehat{d \mu_{k, \frac{y_{1}}{t}, d}}\left(\xi^{\prime}\right) \rho\left(\left|\xi^{\prime}\right|\right) d \xi^{\prime} \\
& =K_{\delta_{t-1}}^{\sigma}(y) \text {, }
\end{aligned}
$$

then

$$
K(y):=\frac{1}{(2 \pi)^{2}} \eta_{1}\left(y_{1}\right) \int_{\mathbb{R}^{2}} e^{i \xi^{\prime} \cdot y^{\prime}} \widehat{d \mu_{k, y_{1}, d}}\left(\xi^{\prime}\right) \rho\left(\left|\xi^{\prime}\right|\right) d \xi^{\prime},
$$

and by integration by parts,

$$
|K(y)| \leq C_{N}(1+|y|)^{-N}, N \in \mathbb{N}
$$

Now, we choose $N$ large enough and (5.44) and (5.45) show that

$$
\left\|M^{k, 0}\right\|_{L^{\infty} \rightarrow L^{\infty}} \leq C
$$

with a constant $C$ which does not depend on $\sigma$. Moreover, scaling by the factor $2^{-k d}$ in the direction of the vector $\sigma$, we see that

$$
\left\|M^{k, 0}\right\|_{L^{1} \rightarrow L^{1, \infty}} \leq C 2^{k d}
$$


Chapter 5. Maximal functions associated with nonisotropic dilations of some classes of hypersurfaces in $\mathbb{R}^{3}$

since we can compare with $2^{k d} M$, where $M$ is the Hardy-Littlewood maximal operator defined by (5.10).

Finally, by interpolation between (5.46) and (5.47), we obtain that

$$
\left\|M^{k, 0}\right\|_{L^{p} \rightarrow L^{p}} \leq C 2^{k d / p}, \quad p>1 .
$$

Then $p>d$ implies that $\sum_{k} 2^{-k}\left\|M^{k, 0}\right\|_{L^{p} \rightarrow L^{p}} \leq C \sum_{k} 2^{k(d / p-1)} \lesssim 1$.

Hence, it suffices to prove that

$$
\left\|M_{j}^{k}\right\|_{L^{p} \rightarrow L^{p}} \leq C_{p} 2^{-j \epsilon(p)} 2^{k d / p}, \quad j \geq 1, \quad d<p<\infty, \text { some } \epsilon(p)>0,
$$

where $M_{j}^{k} f(y):=\sup _{t>0}\left|A_{t, j}^{k} f(y)\right|$.

Since $A_{t, j}^{k}$ is localized to frequencies $\left|\delta_{t}^{\prime} \xi^{\prime}\right| \approx 2^{j}$, we still use Lemma 2.2.4 to prove that

$$
\left\|M_{j}^{k}\right\|_{L^{p} \rightarrow L^{p}} \lesssim\left\|M_{j, l o c}^{k}\right\|_{L^{p} \rightarrow L^{p}}
$$

where $M_{j, l o c}^{k} f(y):=\sup _{t \in[1,2]}\left|A_{t, j}^{k} f(y)\right|$.

Furthermore, a standard application of the method of stationary phase requires us to show the $L^{p}$-boundedness of the operator $\widetilde{\mathcal{M}_{j, l o c}^{k, 1}}$ given by

$$
\begin{aligned}
\widetilde{\mathcal{M}_{j, l o c}^{k, 1}} f(y):= & \sup _{t \in[1,2]}\left|\widetilde{A_{t, j}^{k, 1}} f(y)\right| \\
= & \sup _{t \in[1,2]} \mid \int_{\mathbb{R}} \eta_{1}\left(\frac{x_{1}}{t}\right) \int_{\mathbb{R}^{2}} e^{i Q_{k, x_{1}, d}\left(y^{\prime}, t, \xi^{\prime}\right)} E_{k, x_{1} / t, d}\left(\delta_{t}^{\prime} \xi^{\prime}\right) d x_{1} \\
& \times \beta\left(2^{-j}\left|\delta_{t}^{\prime} \xi^{\prime}\right|\right) f\left(y_{1}-x_{1}, \widehat{\xi^{\prime}}\right) d \xi^{\prime} d x_{1},
\end{aligned}
$$

where

$$
\begin{gathered}
Q_{k, x_{1}, d}\left(y^{\prime}, t, \xi^{\prime}\right):=\left\langle\xi^{\prime},\left(y_{2}, y_{3}-t^{a_{3}} 2^{d k}\right)\right\rangle-t^{a_{3}} \xi_{3} \tilde{\Psi}\left(\frac{x_{1}}{t}, s, \delta\right), \\
\tilde{\Psi}\left(\frac{x_{1}}{t}, s, \delta\right):=\Psi\left(\frac{x_{1}}{t}, \psi\left(\frac{x_{1}}{t}, s, \delta\right), s, \delta\right),
\end{gathered}
$$

and

$$
\Psi\left(x_{1}, x_{2}, s, \delta\right):=-s x_{2}+x_{2}^{d} \Phi\left(x_{1}, \delta x_{2}\right) .
$$

By Lemma 2.2.3, we have

$$
\left\|\widetilde{\mathcal{M}_{j, l o c}^{k, 1}} f\right\|_{L^{p}}^{p} \leq C_{p}\left(\int_{\mathbb{R}^{3}}\left|\rho(t) \widetilde{A_{t, j}^{k, 1}} f(y)\right|^{p}\right)^{1 / p^{\prime}}\left(\int_{\mathbb{R}^{3}}\left|\frac{\partial}{\partial t}\left(\rho(t) \widetilde{A_{t, j}^{k, 1}} f(y)\right)\right|^{p}\right)^{1 / p} .
$$

Since

$$
\begin{aligned}
\left.\mid \frac{\partial}{\partial t} Q_{k, x_{1}, d}\left(y^{\prime}, t, \xi^{\prime}\right)\right) \mid & =\left|-a_{3} t^{a_{3}-1} \xi_{3} 2^{k d}+\xi_{3} \frac{\partial}{\partial t}\left(t^{a_{3}} \tilde{\Psi}\left(\frac{x_{1}}{t}, s, \delta\right)\right)\right| \leq C 2^{k d}\left|\xi_{3}\right| \\
& \approx 2^{d k} 2^{j},
\end{aligned}
$$


Chapter 5. Maximal functions associated with nonisotropic dilations of some classes of hypersurfaces in $\mathbb{R}^{3}$

then $\frac{\partial}{\partial t}\left(\rho(t) \widetilde{A_{t, j}^{k, 1}}\right)$ behaves like $2^{k d} 2^{j} \widetilde{A_{t, j}^{k, 1}}$. Clearly we only consider the $L^{p}$-estimate for the operator $\widehat{A_{t, j}^{k, 1}}$.

The same estimates will follow from the proof of Section 5.3.1, by the assumption $p>d$, finally we obtain

$$
\begin{aligned}
& \sum_{k} 2^{-k} \sum_{j}\left\|\widetilde{\mathcal{M}_{j, l o c}^{k, 1}}\right\|_{L^{p} \rightarrow L^{p}} \\
& \leq C_{p} \sum_{k} 2^{-k} \sum_{j}\left(2^{-j(1 / p+\epsilon(p))}\left\|\eta_{0}\right\|_{L^{1}}\right)^{(p-1) / p}\left(2^{k d} 2^{-j(-1+1 / p+\epsilon(p))}\left\|\eta_{0}\right\|_{L^{1}}\right)^{1 / p} \\
& =C_{p} \sum_{k} 2^{-k(1-d / p)} \sum_{j} 2^{-j p \epsilon(p)}\left\|\eta_{0}\right\|_{L^{1}} \leq C_{p}
\end{aligned}
$$

We have finished the proof of Theorem 5.1.5. 
Chapter 5. Maximal functions associated with nonisotropic dilations of 90 some classes of hypersurfaces in $\mathbb{R}^{3}$ 


\section{Bibliography}

[1] J. G. Bak, Averages over surfaces with infinitely flat points, J. Funct. Anal. 129 (1995), 455-470.

[2] J. Bourgain, Avarages in the plane over convex curves and maximal operators, J. Anal. Math., 47 (1986), 69-85.

[3] M. Cowling, D. Disney, G. Mauceri and D. Müller, Damping oscillatory integrals, Invent. Math., 101 (1990), 237-260.

[4] M. Cowling and G. Mauceri, Inequalities for some maximal functions. II, Trans, Amer. Math. Soc., 296 (1986), 341-365.

[5] M. Cowling and G. Mauceri, Oscillatory integrals and Fourier transforms of surface carried measures, Trans, Amer. Math. Soc., 304 (1987), 53-68.

[6] E. B. Fabes and N. M. Rivière, Singular integrals with mixed homogeneity, Studia Math. 27 (1966), 19-38.

[7] K. J. Falconer, Continuity properties of $\kappa$-plane integrals and Besicovitch sets, Math. Proc. Cambridge Philos. Soc., 87 (1980), 221-226.

[8] C. Fefferman, A note on spherical summation multipliers, Israel. J. Math., 15 (1973), 44-52.

[9] L. Grafakos, Classical and modern Fourier analysis, Prentice-Hall, Upper Saddle River, NJ, 2004.

[10] A. Greenleaf, Principal curvature and harmonic analysis, Indiana U. Math. J., 4 (1981), 519-537.

[11] I. A. Ikromov, M. Kempe and D. Müller, Estimate for maximal operator functions associated with hypersurfaces in $\mathbb{R}^{3}$ and related problems of harmonic analysis, Acta Math., 204 (2010), 151-171. 
[12] A. Iosevich, Maximal operators assciated to families of flat curves in the plane, Duke Math. J., 76 (1994), 633-644.

[13] A. Iosevich and E. Sawyer, Osillatory integrals and maximal averages over homogeneous surfaces, Duke Math. J., 82 (1996), 103-141.

[14] A. Iosevich and E. Sawyer, Maximal averages over surfaces, Adv. Math., 132 (1997), 46-1119.

[15] A. Iosevich, E. Sawyer and A. Seeger, On averaging operators associated with convex hypersurfaces of finite type, J. Anal. Math., 79 (1999), 159-187.

[16] T. Kato, On the Cauchy problem for the (generalized) Kortweg-de Vries equation, Stud. Appl. Math., 8 (1983), 93-128.

[17] D. T. Kung, Local smoothing phenomena for operators failing the cinematic curvature condition, PhD thesis, University of Wisconsin-Madison, 2000.

[18] D. T. Kung, Local smoothing for operators failing the cinematic curvature, J. Math. Anal. Appl., 324 (2006), 460-471.

[19] G. Marletta, Maximal functions with mitigating factors in the plane, J. London Math. Soc., 59 (1999), 647-656.

[20] G. Marletta and F. Ricci, Two-parameter maximal functions associated with homogeneous surfaces in $\mathbb{R}^{n}$, Studia Math., 130 (1998), 53-65.

[21] G. Marletta, F. Ricci and J. Zienkiewicz, Two-parameter maximal functions associate with degenerate homogeneous surface in $\mathbb{R}^{3}$, Studia Math., 130 (1998), 67-75.

[22] J. M. Marstrand, Packing planes in $\mathbb{R}^{3}$, Mathematika, 26 (1979), 180-183.

[23] A. Martini and D. Müller, Spectral multiplier theorems of Euclidean type on further classes of 2-step stratified groups, Proc. London Math. Soc., 2013. ArXiv:1306.0387.

[24] A. Miyachi, On some estimates for the wave equation in $L^{p}$ and $H^{p}$, J. Fac. Sci. Tokyo, 27 (1980), 331354.

[25] G. Mockenhaupt, A. Seeger and C. D. Sogge, Wave front set, local smoothing and Bourgain's circular maximal theorem, Ann. Math., 136 (1992), 207-218. 
[26] G. Mockenhaupt, A. Seeger and C. D. Sogge, Local smoothing of Fourier integral operators and Carleson-Sjolin estimates, J. Amer. Math, 6 (1993), 65-130.

[27] A. Nagel, N. Riviere and S. wainger, A maximal function associated to the curve $\left(t, t^{2}\right)$, Proc. Natl. Acad. Sci. U.S.A, 73 (1976), 1416-1417.

[28] A. Nagel, A. Seeger and S. wainger, Averages over convex hypersurfaces, Amer. J. Math., 115 (1993), 903-927.

[29] D. M. Oberlin and E. M. Stein, Mapping properties of the Radon transform, Indiana Univ. Math. J., 31 (1982), 641-650.

[30] J. Peral, $L^{p}$ estimates for the wave equation, J. Funct. Anal., 36 (1980), 114-115.

[31] A. Ruiz, On a.e. convergence of solutions of hyperbolic equations to $L^{p}$-initial data, Trans. Amer. Math. Soc., 287 (1985), 167-188.

[32] A. Seeger, C. D. Sogge and E. M. Stein, Regularity properties of Fourier integral operators, Ann. Math., 134 (1991), 231-251.

[33] P. Sjölin, Regularity of solutions to the Schrödinger equation, Duke Math. J., 55 (1987), 699-715.

[34] C. D. Sogge, On alomost everywhere convergence to $L^{p}$ data for higher order hyperbolic operators, Proc. Amer. Math. Soc., 100 (1987), 99-103.

[35] C. D. Sogge, Fourier integrals in classical analysis, Cambridge Tracts in Mathematics, vol. 105, Cambridge University Press, Cambridge, 1993.

[36] C. D. Sogge, Maximal operators associtated to hypersurfaces with one nonvanishing principle curvature, in "Fourier analysis and partial differential equations," (J. Garía-Cuerva et al. ed.) CRC Press, Boca Raton (1995), 317-323.

[37] C. D. Sogge and E. M. Stein, Avarages of functions over hypersurfaces in $\mathbb{R}^{n}$, Invent, Math., 82 (1985), 543-556.

[38] E. M. Stein, Singular integrals and differentiability properties of functions, Princeton University Press, 1970.

[39] E. M. Stein, Maximal functions. I. Spherical means., Proc. Nat. Acad. Sci. U.S.A., 73 (1976), 2174-2175.

[40] E. M. Stein, Maximal function: Homogeneous curve, Proc. Natl. Acad. Sci. U.S.A, 73 (1976), 2176-2177. 
[41] E. M. Stein, Hamonic Analysis: real-variable methods, orthogonality and Oscillatory integrals, Princeton University press, 1993.

[42] E. M. Stein, Some problems in harmonic analysis suggested by symmetric spaces and semi-simple groups, Actes, Congrès intern. Math., 1 (1970), 173-189.

[43] E. M. Stein and S. Wainger, Problems in harmonic analysis related to curvature, Bull. Amer. Math. Soc., 84 (1978), 1239-1295.

[44] E. M. Stein and S. Wainger, Maximal functions associated to smooth curves, Proc. Nat. Acad. Sci. U.S.A., 73 (1978), 4295-4296.

[45] R. S. Strichartz, $L^{p}$ estimates for Radon transforms in Euclidean and nonEuclidean spaces, Duke Math. J., 48 (1981), 699-727.

[46] L. Vega, Schrödinger equations: pointwise convergence to the initial data, Proc. Amer. Math. Soc., 102 (1988), 874-878.

[47] E. Zimmermann, On $L^{p}$-estimates for maximal average over hypersurfaces not satisfying the transversality condition, Phd thesis, Christian-Albrechts Universität Bibliothek, 2014. 


\section{Erklärung}

Hiermit versichere ich, dass ich die vorliegende Arbeit, abgesehen von der Beratung durch den Betreuer meiner Promotion, unter Einhaltung der Regeln guter wissenschaftlicher Praxis der Deutschen Forschungsgemeinschaft selbstständig angefertigt habe und keine anderen als die angegebenen Hilfsmittel verwendet habe. Die Arbeit oder Auszüge wurden bislang noch bei keiner anderen Stelle im Rahmen eines Prüfungsverfahrens vorgelegt oder veröffentlicht.

Kiel, den 21. 05. 2015

(Wenjuan Li) 
